\title{
Refinamentos assintóticos em modelos lineares generalizados heteroscedásticos
}

\author{
Fabiana Uchôa Barros
}

TESE APRESENTADA

$\mathrm{AO}$

Instituto de Matemática e Estatística

DA

Universidade de SÃo PaUlo

PARA

OBTENÇÃO DO TÍTULO

$\mathrm{DE}$

Doutor EM CiÊNCIAS

Programa: Estatística

Orientadora: Profa. Dra. Denise Aparecida Botter

Coorientadora: Profa. Dra. Mônica Carneiro Sandoval

Durante o desenvolvimento deste trabalho o autor recebeu auxílio financeiro da CAPES/CNPq

São Paulo, março de 2017 


\section{Refinamentos assintóticos em modelos lineares generalizados heteroscedásticos}

Esta versão da tese contém as correções e alterações sugeridas pela Comissão Julgadora durante a defesa da versão original do trabalho, realizada em 07/03/2017. Uma cópia da versão original está disponível no Instituto de Matemática e Estatística da Universidade de São Paulo.

Comissão Julgadora:

- Profa. Dra. Denise Aparecida Botter (orientadora) - IME-USP

- Profa. Dra. Lúcia Pereira Barroso - IME-USP

- Profa. Dra. Audrey Helen Mariz de Aquino Cysneiros - UFPE

- Prof. Dr. Klaus Leite Pinto Vasconcellos - UFPE

- Prof. Dr. Miguel Angel Uribe Opazo - UNIOESTE 


\section{Resumo}

Nesta tese, desenvolvemos refinamentos assintóticos em modelos lineares generalizados heteroscedásticos (Smyth, 1989). Inicialmente, obtemos a matriz de covariâncias de segunda ordem dos estimadores de máxima verossimilhança corrigidos pelos viés de primeira ordem. Com base na matriz obtida, sugerimos modificações na estatística de Wald. Posteriormente, derivamos os coeficientes do fator de correção tipo-Bartlett para a estatística do teste gradiente. Em seguida, obtemos o coeficiente de assimetria assintótico da distribuição dos estimadores de máxima verossimilhança dos parâmetros do modelo. Finalmente, exibimos o coeficiente de curtose assintótico da distribuição dos estimadores de máxima verossimilhança dos parâmetros do modelo. Analisamos os resultados obtidos através de estudos de simulação de Monte Carlo.

Palavras-chave: Coeficiente de assimetria, Coeficiente de curtose, Correção tipo-Bartlett, Estimadores de máxima verossimilhança, Matriz de covariâncias de segunda ordem, Modelos lineares generalizados heteroscedásticos. 


\section{Abstract}

In this thesis, we have developed asymptotic refinements in heteroskedastic generalized linear models (Smyth, 1989). Initially, we obtain the second-order covariance matrix for the maximum likelihood estimators corrected by the bias of first-order. Based on the obtained matrix, we suggest changes in Wald statistics. In addition, we derive the coefficients of the Bartlett-type correction factor for the statistical gradient test. After, we get asymptotic skewness of the distribution of the maximum likelihood estimators of the model parameters. Finally, we show the asymptotic kurtosis coefficient of the distribution of the maximum likelihood estimators of the model parameters. Monte Carlo simulation studies are developed to evaluate the results obtained.

Keywords: Heteroskedastic generalized linear models, Second-order covariance matrix, Maximum likelihood estimators, Bartlett-type correction, Asymptotic skewness, Asymptotic kurtosis. 


\section{Sumário}

$\begin{array}{ll}\text { Lista de Figuras } & \text { v }\end{array}$

Lista de Tabelas $\quad$ vi

1 Introdução $\quad 1$

1.1 Modelo linear generalizado heteroscedástico . . . . . . . . . . . . . 3

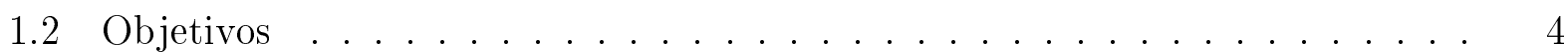

1.3 Organização do Trabalho . . . . . . . . . . . . . . . . 5 5

2 Matriz de Covariâncias de Segunda Ordem $\quad 7$

2.1 Matriz de covariâncias de segunda ordem do EMV . . . . . . . . . . . 7

2.1.1 Matriz de covariâncias de segunda ordem em MLGHs . . . . . . . . 8

2.1.2 Matriz de covariâncias de segunda ordem em MLGs . . . . . . . . . . 11

2.2 Matriz de covariâncias dos estimadores corrigidos . . . . . . . . . . . . 13

2.3 Casos particulares . . . . . . . . . . . . . . . . . . . . 17

2.4 Testes de Wald modificados . . . . . . . . . . . . . . . 18

2.5 Resultados numéricos . . . . . . . . . . . . . . . . 19

2.5.1 Modelo gama . . . . . . . . . . . . . . 20

2.5.2 Modelo normal inversa . . . . . . . . . . . . . 25

2.6 Aplicação . . . . . . . . . . . . . . . . . . . . 28

2.7 Conclusões . . . . . . . . . . . . . . . . . . . 28

3 Aperfeiçoamento do teste gradiente $\quad 30$

3.1 Introdução . . . . . . . . . . . . . . . . . . . . . . . 30

3.2 Teste gradiente aperfeiçoado . . . . . . . . . . . . . . . . 31

3.3 Teste gradiente aperfeiçoado em MLGHs . . . . . . . . . . . . . . 33

3.3.1 Hipótese sobre subconjuntos de $\boldsymbol{\beta}$ e $\boldsymbol{\lambda} \ldots \ldots \ldots 33$

3.3.2 Hipótese sobre um subconjunto de $\boldsymbol{\beta} \ldots \ldots \ldots$. . . . . . . . 38

3.3 .3 Hipótese sobre um subconjunto de $\boldsymbol{\lambda} \ldots \ldots . \ldots . \ldots . \ldots$

3.4 Resultados numéricos . . . . . . . . . . . . . . . . . . . 40

3.4 .1 Modelo gama . . . . . . . . . . . . . . . 42

3.4 .2 Modelo normal inversa . . . . . . . . . . . . . . 44 
3.5 Aplicação . . . . . . . . . . . . . . . . . . . . 57

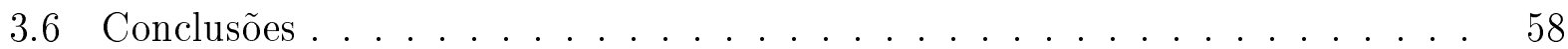

4 Coeficiente de Assimetria $\quad 60$

4.1 Introdução . . . . . . . . . . . . . . . . . . . . . . 6 60

4.2 Coeficiente de assimetria da distribuição do EMV . . . . . . . . . . . . . . 60

4.2.1 Coeficiente de assimetria para a distribuição de $\hat{\boldsymbol{\beta}} \ldots$. . . . . . . 61

4.2 .2 Coeficiente de assimetria para a distribuição de $\hat{\boldsymbol{\lambda}} \ldots$. . . . . . . 62

4.3 Resultados numéricos . . . . . . . . . . . . . . . . . . . 62

4.4 Conclusões . . . . . . . . . . . . . . . . . . . 63

5 Coeficiente de Curtose $\quad 69$

5.1 Introdução . . . . . . . . . . . . . . . . . . . . . . . 69

5.2 Coeficiente de curtose da distribuição do EMV . . . . . . . . . . . . 70

5.2 .1 Coeficiente de curtose para a distribuição de $\hat{\boldsymbol{\beta}}$. . . . . . . . . . 71

5.2 .2 Coeficiente de curtose para a distribuição de $\hat{\boldsymbol{\lambda}} \ldots \ldots . . \ldots 76$

5.3 Casos particulares . . . . . . . . . . . . . . . . . . 79

5.4 Resultados numéricos . . . . . . . . . . . . . . . 85

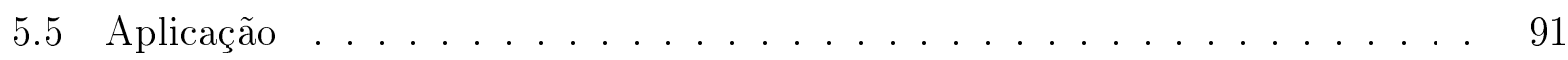

5.6 Conclusões . . . . . . . . . . . . . . . . . . . . . 91

6 Conclusões e Pesquisas Futuras $\quad 92$

$\begin{array}{ll}\text { A Identidades de Bartlett e Cumulantes } & 94\end{array}$

A.1 Identidades de Bartlett . . . . . . . . . . . . . . . . 94

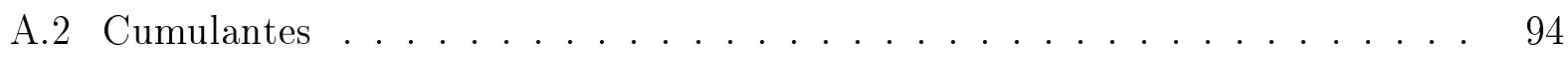

$\begin{array}{ll}\text { B Fator de correção tipo-Bartlett } & 97\end{array}$

C Obtenção das quantidades $A_{40}, A_{32}, A_{33}$ e $A_{22}$ do coeficiente de curtose 102

C.1 Obtenção de $A_{32}$ para o coeficiente de curtose da distribuição . . . . . . . . 102

C.2 Obtenção de $A_{32}$ para o coeficiente de curtose da distribuição . . . . . . . . 106

$\begin{array}{lr}\text { Referências Bibliográficas } & 111\end{array}$ 


\section{Lista de Figuras}

2.1 Quantis assintóticos versus quantis empíricos das estatísticas $W, W_{1}$ e $W_{2}$ modelo gama com $p=2, p_{1}=1$ e $q=2 \ldots \ldots \ldots$

2.2 Quantis assintóticos versus quantis empíricos das estatísticas $W, W_{1}$ e $W_{2}$ modelo normal inversa com $p=2, p_{1}=1$ e $q=2 \ldots \ldots . \ldots 25$

3.1 Discrepâncias relativas de quantis - modelo gama com $n=40, p=3$ e $q=3 . \quad 44$

3.2 Discrepâncias relativas de quantis - modelo normal inversa com $n=40, p=3$

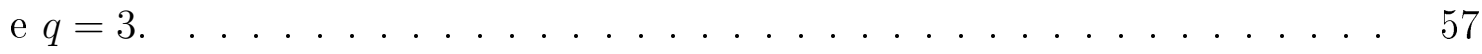

4.1 Densidades estimada e normal; modelo gama e covariável $\mathcal{U}(0,1)$. . . . . . 65

4.2 Densidades estimada e normal; modelo normal inversa e covariável $\mathcal{U}(0,1)$. $\quad 66$

4.3 Densidades estimada e normal; modelo gama e covariável Exponencial(6). . . 67

4.4 Densidades estimada e normal; modelo normal inversa e covariável Exponen-

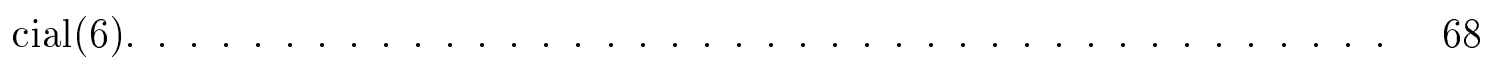

5.1 Densidades estimada e normal; modelo gama e covariável $\mathcal{U}(0,1)$. . . . . . 87

5.2 Densidades estimada e normal; modelo normal inversa e covariável $\mathcal{U}(0,1) . \quad$. $\quad 88$

5.3 Densidades estimada e normal; modelo gama e covariável Weibull $(2,2)$. . . 89

5.4 Densidades estimada e normal; modelo normal inversa e covariável Weibull $(2,2) .90$ 


\section{Lista de Tabelas}

1.1 Derivação de algumas quantidades para distribuições da família exponencial. 4

$2.1 \overline{\operatorname{Cov}}_{1}(\widehat{\boldsymbol{\theta}}), \overline{\operatorname{Cov}}_{2}(\overline{\boldsymbol{\theta}})$ e covariâncias amostrais para a distribuição gama. . . . . . 23

2.2 Taxas de rejeição para $\mathcal{H}_{0}: \beta_{2}=1$; distribuição gama. . . . . . . . . . . . 24

2.3 Taxas de rejeição para $\mathcal{H}_{0}: \lambda_{2}=1$; distribuição gama. . . . . . . . . . . 24

2.4 Taxas de rejeição para $\mathcal{H}_{0}: \beta_{2}=1, \lambda_{2}=1$; distribuição gama. . . . . . . . 24

2.5 Poder para $\mathcal{H}_{1}: \beta_{2}=\epsilon ; n=30, \alpha=10 \%$ e distribuição gama. . . . . . . . 24

$2.6 \overline{\operatorname{Cov}}_{1}(\widehat{\boldsymbol{\theta}}), \overline{\operatorname{Cov}}_{2}(\overline{\boldsymbol{\theta}})$ e covariâncias amostrais para a distribuição normal inversa. 26

2.7 Taxas de rejeição para $\mathcal{H}_{0}: \beta_{1}=1$; distribuição normal inversa. . . . . . . . 27

2.8 Taxas de rejeição para $\mathcal{H}_{0}: \lambda_{2}=1$; distribuição normal inversa. . . . . . . . 27

2.9 Taxas de rejeição para $\mathcal{H}_{0}: \beta_{2}=1, \lambda_{2}=1$; distribuição normal inversa. . . . 27

2.10 Poder para $\mathcal{H}_{1}: \beta_{2}=\lambda_{2}=\epsilon ; n=30, \alpha=10 \%$ e distribuição normal inversa. $\quad 27$

2.11 Estimativas dos parâmetros e respectivos erros padrões . . . . . . . . . 28

2.12 Valores das estatísticas de teste e valores-p para testar $\mathcal{H}_{0}: \lambda_{2}=0 . \quad \ldots \quad 28$

3.1 Taxas de rejeição para $\mathcal{H}_{0}: \beta_{p}=0$; distribuição gama com $n=30$ e $q=2 . \quad$. 45

3.2 Taxas de rejeição para $\mathcal{H}_{0}: \beta_{p}=0$; distribuição gama com $n=30$ e $p=2 . \quad$. 46

3.3 Taxas de rejeição para $\mathcal{H}_{0}: \beta_{p}=0$; distribuição gama com $p=3$ e $q=3$. . . 47

3.4 Taxas de rejeição para $\mathcal{H}_{0}: \lambda_{2}=\ldots=\lambda_{q}=0$; distribuição gama com $p=2$

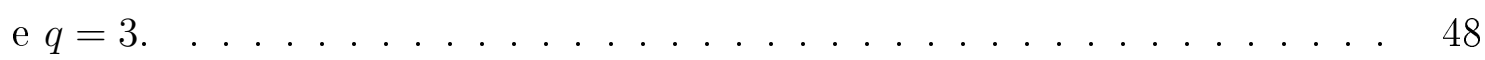

3.5 Taxas de rejeição para $\mathcal{H}_{0}: \lambda_{2}=\ldots=\lambda_{q}=0$; distribuição gama com $p=1 . \quad 49$

3.6 Poder para $\mathcal{H}_{1}: \lambda_{2}=\epsilon ; n=30, p=1, q=2, \alpha=10 \%$ e distribuição gama. . 50

3.7 Taxas de rejeição para $\mathcal{H}_{0}: \beta_{p}=0$; distribuição normal inversa com $n=30 \mathrm{e}$ $q=2$.

3.8 Taxas de rejeição para $\mathcal{H}_{0}: \beta_{p}=0$; distribuição normal inversa com $n=30 \mathrm{e}$ $p=2 \ldots \ldots \ldots \ldots \ldots \ldots \ldots \ldots \ldots \ldots \ldots \ldots \ldots \ldots \ldots \ldots$

3.9 Taxas de rejeição para $\mathcal{H}_{0}: \beta_{p}=0$; distribuição normal inversa com $p=3 \mathrm{e}$ $q=3$.

3.10 Taxas de rejeição para $\mathcal{H}_{0}: \lambda_{2}=\ldots=\lambda_{q}=0$; distribuição normal inversa $\operatorname{com} p=2$ e $q=3 \ldots \ldots \ldots \ldots \ldots$ 
3.11 Taxas de rejeição para $\mathcal{H}_{0}: \lambda_{2}=\ldots=\lambda_{q}=0$; distribuição normal inversa $\operatorname{com} p=1 \ldots \ldots \ldots \ldots \ldots \ldots \ldots \ldots$

3.12 Poder para $\mathcal{H}_{1}: \lambda_{2}=\epsilon ; n=30, p=1, q=2, \alpha=10 \%$ e distribuição normal

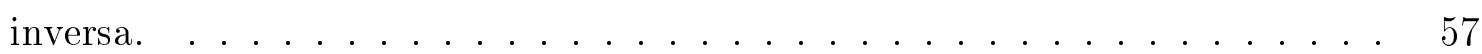

3.13 Estimativas dos parâmetros. . . . . . . . . . . . . 58

3.14 Valores das estatísticas de teste e p-valores para testar $\mathcal{H}_{0}: \lambda_{2}=0 . \ldots 58$

4.1 Coeficiente de assimetria amostral e de ordem $n^{-1 / 2}$ e covariável $\mathcal{U}(0,1)$. . 64

4.2 Coeficiente de assimetria amostral e de ordem $n^{-1 / 2}$ e covariável Exponencial(6). 64

5.1 Coeficiente de excesso de curtose amostral e de ordem $n^{-1}$ e covariável $\mathcal{U}(0,1) .86$

5.2 Coeficiente de excesso de curtose amostral e de ordem $n^{-1}$ e covariável Weibull $(2,2) .86$

5.3 Estimativas dos parâmetros, assimetria e curtose estimada. . . . . . . . . . . 91 


\section{Capítulo 1}

\section{Introdução}

Modelos de regressão são modelos matemáticos que buscam explicar o comportamento de uma variável resposta por meio de variáveis explicativas. Estes modelos são bastante utilizados em diversas áreas do conhecimento, tais como, Engenharia, Medicina, Economia, etc.

Um fato bastante comum nos modelos de regressão é a suposição de normalidade para a variável resposta. Quando essa suposição não é satisfeita, é comum se tentar algum tipo de transformação na variável resposta com o objetivo de obter uma nova variável que seja normalmente distribuída. Dependendo, porém, da transformação utilizada o modelo pode vir a ser inviável devido à complexidade de sua interpretação.

Uma extensão dos modelos normais lineares foi proposta por Nelder e Wedderburn (1972). Eles propuseram os modelos lineares generalizados (MLGs). Estes modelos permitem tanto que a relação entre a média da variável resposta e o preditor linear seja mais flexível quanto que a distribuição da variável resposta pertença à família exponencial. Como exemplo de distribuições pertencentes a essa família, temos: normal, normal inversa, gama, binomial e Poisson. Portanto, os MLGs podem ser utilizados para modelar dados simétricos, assimétricos positivos, binários e de contagem.

A possibilidade de modelar o parâmetro de dispersão em MLGs foi apresentada inicialmente por Pregibon (1984). Esta modelagem só foi formalizada cinco anos depois por Smyth (1989) e deu origem aos modelos lineares generalizados heteroscedásticos (MLGHs).

Iremos agora, destacar algumas contribuições em termos de teoria assintótica na classe dos MLGs e MLGHs.

A estimação dos parâmetros, na maioria das vezes, é feita através do método de máxima verossimilhança, que fornece, em geral, estimativas viesadas para os valores verdadeiros dos parâmetros. Em virtude disso, correções do viés têm sido bastante estudadas na literatura estatística com o objetivo de melhorar a qualidade dessas estimativas, principalmente em se tratando de amostras pequenas. A partir da expressão geral de Cox e Snell (1968) para os vieses de ordem $n^{-1}$, em que $n$ é o tamanho da amostra, dos estimadores de máxima verossimilhança (EMVs) nos modelos multiparamétricos, Cordeiro e McCullagh 
(1991) e Botter e Cordeiro (1998) obtiveram expressões matriciais para os vieses em MLGs e MLGHs, respectivamente.

Os testes frequentemente utilizados em grandes amostras baseiam-se nas estatísticas da razão de verossimilhanças (Wilks, 1938), Wald (Wald, 1943) e escore (Rao, 1947). Uma estatística alternativa para estes testes em grandes amostras foi proposta por Terrell (2002) e denominada de estatística gradiente. Esta estatística, assim como as outras (razão de verossimilhanças, Wald e escore), possui distribuição assintótica qui-quadrado $\left(\chi^{2}\right)$ sob a hipótese nula. Entretanto, sabemos que em pequenas amostras a aproximação $\chi^{2}$ usual para a distribuição destas estatísticas pode não ser satisfatória. Com o intuito de melhorar esta aproximação, houve a necessidade de sugerir métodos de aperfeiçoamento dos testes por meio de estatísticas modificadas, como por exemplo, fatores de correção de Bartlett e tipo-Bartlett e ajuste de Skovgaard.

Com base no trabalho de Lawley (Lawley, 1956), correções de Bartlett para a estatística da razão de verossimilhanças em MLGs foram obtidas por Cordeiro (1983, 1987) considerando o parâmetro de dispersão conhecido e desconhecido, respectivamente. Estes resultados foram estendidos por Botter e Cordeiro (1997) para os MLGHs. Um outro tipo de correção para a estatística da razão de verossimilhanças foi obtido por Ferrari e Cysneiros (2008), por meio do ajuste de Skovgaard (Skovgaard, 2001), para os modelos não lineares da família exponencial heteroscedásticos, os quais generalizam os MLGHs. Baseado no trabalho de Cordeiro e Ferrari (1991), correções tipo-Bartlett para a estatística escore em MLGs foram obtidas por Cordeiro et al. (1993) e Cribari-Neto e Ferrari (1995) para o caso em que o parâmetro de dispersão é conhecido e desconhecido, respectivamente. Estes resultados foram generalizados por Cordeiro et al. (2003) para os MLGHs. Recentemente, Vargas et al. (2013) sugeriram uma estatística gradiente corrigida por um fator de correção tipo-Bartlett. A partir de seus resultados, eles derivaram expressões de forma fechada para o fator de correção tipo-Bartlett que define a estatística gradiente corrigida na classe dos MLGs. Maiores informações sobre a estatística gradiente podem ser encontradas em Lemonte (2016).

Cordeiro (2004) e Cordeiro et al. (2006) obtiveram uma fórmula matricial para a matriz de covariâncias de segunda ordem dos EMVs em MLGs considerando o parâmetro de dispersão conhecido e desconhecido, respectivamente. Barroso et al. (2013) generalizaram estes resultados para os MLGHs. Recentemente, Cordeiro et al. (2014) obtiveram a matriz de covariâncias de segunda ordem para os EMVs corrigidos pelo viés de ordem $n^{-1}$ em MLGs considerando o parâmetro de dispersão conhecido. A partir da expressão obtida, os autores sugeriram uma modificação na estatística de Wald, a qual melhorou o seu desempenho.

Aproximações de ordens $n^{-2}$ e $n^{-3}$ para o terceiro e quarto momentos centrais da distribuição dos EMVs foram dadas por Bowman e Shenton $(1998,1999)$, respectivamente. A partir destas aproximações, eles obtiveram os coeficientes de assimetria e curtose até as ordens $n^{-1 / 2}$ e $n^{-1}$, respectivamente, das distribuições dos EMVs. Utilizando Bowman e Shenton (1998), Cordeiro e Cordeiro (2001) obtiveram o coeficiente de assimetria de ordem $n^{-1 / 2}$ 
das distribuições dos EMVs em MLGs.

\subsection{Modelo linear generalizado heteroscedástico}

Sejam $Y_{1}, \ldots, Y_{n}$ variáveis aleatórias independentes com cada $Y_{\ell}$ tendo função densidade de probabilidade (ou função de probabilidade) dada por

$$
\pi\left(y ; \theta_{\ell}, \phi_{\ell}\right)=\exp \left\{\phi_{\ell}\left[y \theta_{\ell}-b\left(\theta_{\ell}\right)+c(y)\right]+a(y)+d\left(\phi_{\ell}\right)\right\}
$$

em que $a(\cdot), b(\cdot), c(\cdot)$ e $d(\cdot)$ são funções conhecidas. A média e a variância de $Y_{\ell}$ são, $\mathrm{E}\left(Y_{\ell}\right)=$ $\mu_{\ell}=\mathrm{d} b\left(\theta_{\ell}\right) / \mathrm{d} \theta_{\ell}$ e $\operatorname{Var}\left(Y_{\ell}\right)=\phi_{\ell}^{-1} V_{\ell}$, em que $V_{\ell}=\mathrm{d} \mu_{\ell} / \mathrm{d} \theta_{\ell}$ é denominada função de variância e $\theta_{\ell}=\int V_{\ell}^{-1} \mathrm{~d} \mu_{\ell}=q\left(\mu_{\ell}\right)$, sendo $q\left(\mu_{\ell}\right)$ uma função um-a-um de $\mu_{\ell}$, conhecida, que varia em um subconjunto dos reais. Os parâmetros $\theta_{\ell}$ e $\phi_{\ell}>0$ são chamados parâmetros canônico e de precisão, respectivamente. O parâmetro de dispersão da distribuição é o inverso de $\phi_{\ell}$.

Os MLGHs são definidos por (1.1) e pelas componentes sistemáticas

$$
t_{1}(\boldsymbol{\mu})=\boldsymbol{\eta}=\boldsymbol{X} \boldsymbol{\beta} \quad \text { e } \quad t_{2}(\boldsymbol{\phi})=\boldsymbol{\tau}=\boldsymbol{S} \boldsymbol{\lambda}
$$

em que $t_{1}(\cdot)$ é uma função invertível, conhecida e duas vezes diferenciável denominada função de ligação da média $\boldsymbol{\mu}=\left(\mu_{1}, \ldots, \mu_{n}\right)^{\top}, \boldsymbol{\eta}=\left(\eta_{1}, \ldots, \eta_{n}\right)^{\top}$ é o preditor linear da média, $\boldsymbol{X}=\left(\boldsymbol{x}_{1}, \ldots, \boldsymbol{x}_{n}\right)^{\top}$ é uma matriz especificada de dimensão $n \times p$ de posto completo $p<n$, com $\boldsymbol{x}_{\ell}=\left(x_{\ell 1}, \ldots, x_{\ell p}\right)^{\top}$ sendo a sua $\ell$-ésima linha, $\boldsymbol{\beta}=\left(\beta_{1}, \ldots, \beta_{p}\right)^{\top}$ é um vetor de parâmetros desconhecidos a serem estimados, $t_{2}(\cdot)$ é uma função invertível, conhecida, duas vezes diferenciável denominada função de ligação da dispersão $\phi=\left(\phi_{1}, \ldots, \phi_{n}\right)^{\top}, \boldsymbol{\tau}=\left(\tau_{1}, \ldots, \tau_{n}\right)^{\top}$ é o preditor linear da dispersão, $\boldsymbol{S}=\left(\boldsymbol{s}_{1}, \ldots, \boldsymbol{s}_{n}\right)^{\top}$ é uma matriz especificada de dimensão $n \times q$ de posto completo $q<n$, com $\boldsymbol{s}_{\ell}=\left(s_{\ell 1}, \ldots, s_{\ell q}\right)^{\top}$ sendo a sua $\ell$-ésima linha, $\boldsymbol{\lambda}=\left(\lambda_{1}, \ldots, \lambda_{q}\right)^{\top}$ é um vetor de parâmetros desconhecidos a serem estimados.

Seja $\ell(\boldsymbol{\beta}, \boldsymbol{\lambda})$ o logaritmo da função de verossimilhança do modelo definido por (1.1) e (1.2) dado o conjunto de observações $\boldsymbol{y}=\left(y_{1}, \ldots, y_{n}\right)^{\top}$. Temos então

$$
\ell(\boldsymbol{\beta}, \boldsymbol{\lambda})=\sum_{\ell=1}^{n} \phi_{\ell}\left[y_{\ell} \theta_{\ell}-b\left(\theta_{\ell}\right)+c\left(y_{\ell}\right)\right]+\sum_{\ell=1}^{n}\left[a\left(y_{\ell}\right)+d\left(\phi_{\ell}\right)\right] .
$$

Assumimos que o logaritmo da função de verossimilhança satisfaz as condições de regularidade (Cox e Hinkley, 1974, Cap. 9) no que diz respeito a todas as derivadas em relação a $\boldsymbol{\beta}$ e $\boldsymbol{\lambda}$ até a quarta ordem. A seguinte notação será utilizada a partir de agora, $d_{k \ell}=\mathrm{d}^{k} d\left(\phi_{\ell}\right) / \mathrm{d} \phi_{\ell}^{k}$ e $\phi_{k \ell}=\mathrm{d}^{k} \phi_{\ell} / \mathrm{d} \tau_{\ell}^{k}$ para $k=1, \ldots, 4$ e $\ell=1, \ldots, n$.

Na Tabela 1.1 são apresentadas algumas quantidades para distribuições pertencentes à família exponencial. A quantidade $\Gamma(\phi)=\int_{0}^{\infty} t^{\phi-1} e^{-t} d t$ é a função gama, $\psi(\phi)=\Gamma^{\prime}(\phi) / \Gamma(\phi)$ é a função digama e $\psi^{(r)}(\phi)=\mathrm{d}^{r} \psi(\phi) / \mathrm{d} \phi^{r}$ para $r=1,2$ e 3 é a $r$-ésima derivada da função 
digama.

Tabela 1.1: Derivação de algumas quantidades para distribuiçôes da familia exponencial.

\begin{tabular}{cccc}
\hline & Normal: $\mathrm{N}\left(\mu, \sigma^{2}\right)$ & Normal Inversa: NI $(\mu, \phi)$ & Gama: $\mathrm{G}(\mu, \phi)$ \\
\hline$\theta$ & $\mu$ & $-1 / 2 \mu^{2}$ & $-1 / \mu$ \\
$b(\theta)$ & $\theta^{2} / 2$ & $-\sqrt{-2 \theta}$ & $-\log (-\theta)$ \\
$\phi$ & $\sigma^{-2}$ & $\phi$ & $\mathrm{E}^{2}(Y) / \operatorname{Var}(Y)$ \\
$V(\mu)$ & 1 & $\mu^{3}$ & $\mu^{2}$ \\
$d(\phi)$ & $1 / 2 \log \phi$ & $1 / 2 \log \phi$ & $\phi \log \phi-\log \Gamma(\phi)$ \\
$d_{1}$ & $1 / 2 \phi$ & $1 / 2 \phi$ & $\log \phi+1-\psi(\phi)$ \\
$d_{2}$ & $-1 / 2 \phi^{2}$ & $-1 / 2 \phi^{2}$ & $(1 / \phi)-\psi^{(1)}(\phi)$ \\
$d_{3}$ & $1 / \phi^{3}$ & $1 / \phi^{3}$ & $\left(-1 / \phi^{2}\right)-\psi^{(2)}(\phi)$ \\
$d_{4}$ & $-3 / \phi^{4}$ & $-3 / \phi^{4}$ & $\left(2 / \phi^{3}\right)-\psi^{(3)}(\phi)$ \\
\hline
\end{tabular}

O vetor escore, obtido diferenciando-se o logaritmo da função de verossimilhança com respeito aos parâmetros desconhecidos, é dado por $\boldsymbol{U}(\boldsymbol{\beta}, \boldsymbol{\lambda})=\left(\boldsymbol{U}_{\beta}(\boldsymbol{\beta}, \boldsymbol{\lambda})^{\top}, \boldsymbol{U}_{\lambda}(\boldsymbol{\beta}, \boldsymbol{\lambda})^{\top}\right)^{\top}$, em que

$$
\boldsymbol{U}_{\beta}(\boldsymbol{\beta}, \boldsymbol{\lambda})=\boldsymbol{X}^{\top} \boldsymbol{\Phi} \boldsymbol{W}^{1 / 2} \boldsymbol{V}^{-1}(\boldsymbol{y}-\boldsymbol{\mu}) \quad \text { e } \quad \boldsymbol{U}_{\lambda}(\boldsymbol{\beta}, \boldsymbol{\lambda})=\boldsymbol{S}^{\top} \boldsymbol{\Phi}_{1} \boldsymbol{\nu}
$$

$\operatorname{com} \boldsymbol{W}=\operatorname{diag}\left\{w_{1}, \ldots, w_{n}\right\}$ sendo $w_{\ell}=V_{\ell}^{-1}\left(\mathrm{~d} \mu_{\ell} / \mathrm{d} \eta_{\ell}\right)^{2}, \boldsymbol{V}=\operatorname{diag}\left\{V_{1}, \ldots, V_{n}\right\}, \boldsymbol{\Phi}=$ $\operatorname{diag}\left\{\phi_{1}, \ldots, \phi_{n}\right\}, \boldsymbol{\Phi}_{1}=\operatorname{diag}\left\{\phi_{11}, \ldots, \phi_{1 n}\right\}$ e $\boldsymbol{\nu}=\left(\nu_{1}, \ldots, \nu_{n}\right)^{\top} \operatorname{com} \nu_{\ell}=y_{\ell} \theta_{\ell}-b\left(\theta_{\ell}\right)+$ $c\left(y_{\ell}\right)+d_{1 \ell}$.

A matriz de informação de Fisher é dada por

$$
\boldsymbol{K}=\boldsymbol{K}(\boldsymbol{\beta}, \boldsymbol{\lambda})=\left(\begin{array}{cc}
\boldsymbol{K}_{\beta} & \mathbf{0} \\
\mathbf{0} & \boldsymbol{K}_{\lambda}
\end{array}\right)
$$

em que $\boldsymbol{K}_{\beta}=\boldsymbol{X}^{\top} \boldsymbol{W} \boldsymbol{\Phi} \boldsymbol{X}$ e $\boldsymbol{K}_{\lambda}=-\boldsymbol{S}^{\top} \boldsymbol{D}_{2} \boldsymbol{\Phi}_{1}^{2} \boldsymbol{S} \operatorname{com} \boldsymbol{D}_{2}=\operatorname{diag}\left\{d_{21}, \ldots, d_{2 n}\right\}$.

Observe que a matriz de informação de Fisher é uma matriz bloco diagonal e portanto os parâmetros $\boldsymbol{\beta}$ e $\boldsymbol{\lambda}$ são globalmente ortogonais (Cox e Reid, 1987). Sob as condições de regularidade referenciadas anteriormente, temos para um tamanho de amostra grande que $\left(\hat{\boldsymbol{\beta}}^{\top}, \hat{\boldsymbol{\lambda}}^{\top}\right)^{\top}$ é aproximadamente distribuído como $N_{p+q}\left(\left(\boldsymbol{\beta}^{\top}, \boldsymbol{\lambda}^{\top}\right)^{\top}, \boldsymbol{K}^{-1}(\boldsymbol{\beta}, \boldsymbol{\lambda})\right)$. Segue destes fatos a independência assintótica entre $\hat{\boldsymbol{\beta}}$ e $\hat{\boldsymbol{\lambda}}$. Estes estimadores satisfazem as equações $\boldsymbol{U}(\hat{\boldsymbol{\beta}}, \hat{\boldsymbol{\lambda}})=0$ e para resolvê-las é necessário recorrer a métodos iterativos (Nocedal e Wright, 2006) como escore de Fisher ou, equivalentemente, mínimos quadrados reponderados.

\subsection{Objetivos}

Esta tese visa a obtenção de alguns resultados na classe dos MLGHs, são eles : 
1. A matriz de covariâncias de segunda ordem dos estimadores de máxima verossimilhança corrigidos pelo viés de primeira ordem;

2. O fator de correção tipo-Bartlett para a estatística gradiente;

3. O coeficiente de assimetria até a ordem $n^{-1 / 2}$ da distribuição dos estimadores de máxima verossimilhança;

4. O coeficiente de curtose até a ordem $n^{-1}$ da distribuição dos estimadores de máxima verossimilhança.

\subsection{Organização do Trabalho}

No Capítulo 2, obtemos a matriz de covariâncias de segunda ordem dos estimadores de máxima verossimilhança corrigidos pelo viés de primeira ordem em modelos lineares generalizados heteroscedásticos. A partir da matriz obtida, sugerimos modificações na estatística de Wald. Através de estudos de simulação, comparamos a aproximação da matriz de covariâncias de primeira e segunda ordem dos estimadores corrigidos em relação a matriz de covariâncias amostrais destes estimadores. Também comparamos o desempenho da estatística de Wald usual, suas versões modificadas e a estatística de Wald bootstrap. Realizamos uma aplicação em um conjunto de dados reais e, por fim, apresentamos algumas conclusões.

No Capítulo 3, deduzimos os coeficientes do fator de correção tipo-Bartlett para a estatística do teste gradiente em MLGHs. Por meio de simulação comparamos o desempenho dos seguintes testes: testes da razão de verossimilhanças usual, ajustada e corrigida, testes escore usual e corrigido, testes Wald usual e modificado, teste gradiente usual e corrigido (desenvolvido no respectivo capítulo), testes da razão de verossimilhanças, escore, Wald e gradiente bootstrap. Aplicamos o resultado obtido a um conjunto de dados reais e exibimos certas conclusões.

No Capítulo 4, obtemos o coeficiente de assimetria até ordem $n^{-1 / 2}$ da distribuição dos estimadores de máxima verossimilhança dos parâmetros dos modelos lineares generalizados heteroscedásticos. Mediante estudo de simulação, discutimos a aproximação normal para a distribuição dos EMVs dos parâmetros dos modelos e apresentamos algumas conclusões.

No Capítulo 5, desenvolvemos o coeficiente de curtose até ordem $n^{-1}$ da distribuição dos estimadores de máxima verossimilhança dos parâmetros dos modelos lineares generalizados heteroscedásticos. Por meio de simulação, avaliamos a aproximação normal para a distribuição do EMV dos parâmetros dos modelos. Aplicação a um conjunto de dados reais e conclusões são apresentadas.

Finalmente, no Capítulo 6, apresentamos algumas conclusões e damos algumas sugestões de pesquisas que poderão ser desenvolvidas futuramente. 
Os estudos de simulação e os gráficos apresentados neste trabalho foram construídos utilizando as linguagens matriciais de programação Ox (Doornik, 2006) e R (R Development Core, 2009). 


\section{Capítulo 2}

\section{Matriz de Covariâncias de Segunda Ordem}

Neste capítulo, desenvolvemos uma expressão para a matriz de covariâncias de segunda ordem dos EMVs corrigidos pelo viés de ordem $n^{-1}$ em MLGHs. Com base na matriz obtida e nos estimadores corrigidos, sugerimos modificações na estatística de Wald. Realizamos estudos de simulação para analisar o desempenho dessa matriz bem como comparar a performance do teste de Wald usual, suas versões modificadas e o teste de Wald bootstrap. Por fim, aplicamos o resultado obtido a um conjunto de dados reais.

\subsection{Matriz de covariâncias de segunda ordem do EMV}

A matriz de covariâncias de segunda ordem do EMV corrigido pelo viés de ordem $n^{-1}$ depende da matriz de covariâncias de segunda ordem do EMV.

Ao longo da última década, diversos trabalhos relacionados à matriz de covariâncias de segunda ordem foram desenvolvidos para diversos modelos estatísticos. Esses trabalhos baseavam-se na função geradora de cumulantes do EMV até a ordem $n^{-2}$ obtida por Peers e Iqbal (1985) que possibilitava encontrar a matriz de covariâncias de segunda ordem do EMV. Dentre os trabalhos que utilizaram Peers e Iqbal (1985) para obter essa matriz podemos citar: Cordeiro (2004) e Cordeiro et al. (2006) para os MLGs considerando o parâmetro de dispersão conhecido e desconhecido, respectivamente; Cordeiro e Santana (2008) para os modelos não lineares da família exponencial; Rocha et al. (2010) para os modelos de dispersão e Barroso et al. (2013) para os MLGHs. Shenton e Bowman (1977, p. 67) também desenvolveram expressões para a covariância de segunda ordem que diferem daquelas obtidas por Peers e Iqbal (1985). Magalhães (2016), por sua vez, refez as contas para as expressões da covariância de segunda ordem e obteve os mesmos resultados de Shenton e Bowman (1977, p. 67).

De acordo com Shenton e Bowman (1977, p. 67) a covariância de segunda ordem entre 
quaisquer dois estimadores $\hat{\theta}_{i}$ e $\hat{\theta}_{j}$ pode ser expressa como

$$
\operatorname{Cov}_{2}\left(\hat{\theta}_{i}, \hat{\theta}_{j}\right)=-\kappa^{i j}+\sigma_{i j}
$$

em que $-\kappa^{i j}$ representa o $(i, j)$-ésimo elemento da inversa da matriz de informação de Fisher e

$$
\sigma_{i j}=\sigma_{i j}^{(1)}+\sigma_{i j}^{(2)}+\sigma_{i j}^{(3)}
$$

com

$$
\begin{gathered}
\sigma_{i j}^{(1)}=-\kappa^{i a} \kappa^{j b} \kappa^{c d}\left(\kappa_{a b c d}+(1 / 2) \kappa_{a, b c d}+(1 / 2) \kappa_{a c d, b}+2 \kappa_{a b c, d}+\kappa_{a, b c, d}+\kappa_{a c, b, d}+3 \kappa_{a c, b d}\right) \\
\sigma_{i j}^{(2)}=\kappa^{i a} \kappa^{j r} \kappa^{b s} \kappa^{c t}\left[(3 / 2) \kappa_{a b c} \kappa_{r s t}+2 \kappa_{a b, c} \kappa_{r s t}+2 \kappa_{a b c} \kappa_{r s, t}+(1 / 2) \kappa_{a, b c} \kappa_{r s t}+(1 / 2) \kappa_{a b c} \kappa_{r, s t}\right. \\
\left.+\kappa_{a b, c} \kappa_{r, s t}+\kappa_{a, b c} \kappa_{r s, t}+(1 / 2) \kappa_{a b, c} \kappa_{r t, s}+(1 / 2) \kappa_{a c, b} \kappa_{r s, t}\right] \\
\sigma_{i j}^{(3)}=\kappa^{i a} \kappa^{j b} \kappa^{r s} \kappa^{c t}\left(\kappa_{a, b c} \kappa_{r, s t}+\kappa_{a c, b} \kappa_{r, s t}+(1 / 2) \kappa_{a, b c} \kappa_{r s t}+(1 / 2) \kappa_{a c, b} \kappa_{r s t}\right. \\
\left.+\kappa_{a b c} \kappa_{r s t}+2 \kappa_{a b c} \kappa_{r, s t}\right)
\end{gathered}
$$

em que os cumulantes conjuntos das derivadas do logaritmo da função de verossimilhança são dados por $\kappa_{r s}=\mathrm{E}\left(\partial^{2} \ell / \partial \theta_{r} \theta_{s}\right), \kappa_{r, s}=\mathrm{E}\left(\partial \ell / \partial \theta_{r} \partial \ell / \partial \theta_{s}\right), \kappa_{r s t}=\mathrm{E}\left(\partial^{3} \ell / \partial \theta_{r} \theta_{s} \theta_{t}\right), \kappa_{r, s t}=$ $\mathrm{E}\left(\partial \ell / \partial \theta_{r} \partial^{2} \ell / \partial \theta_{s} \theta_{t}\right)$, e assim por diante. Definimos as derivadas dos cumulantes conjuntos por $\kappa_{r s}^{(t)}=\partial \kappa_{r s} / \partial \theta_{t}, \kappa_{r s}^{(t u)}=\partial \kappa_{r s} / \partial \theta_{t} \partial \theta_{u}$, etc. Todos os $\kappa^{\prime}$ 's referem-se a um total sobre a amostra e são, em geral, de ordem $n$.

Como um dos objetivos desse capítulo é obter a matriz de covariâncias de segunda ordem dos EMVs corrigidos pelo viés de ordem $n^{-1}$ em MLGHs, a qual depende da $\mathrm{Cov}_{2}$, resolvemos então, utilizando as expressões (2.1)-(2.5) corrigir os resultados obtidos para a $\mathrm{Cov}_{2}$ em MLGHs (Barroso et al., 2013) e seus casos particulares (Cordeiro, 2004; Cordeiro et al. ,2006).

\subsubsection{Matriz de covariâncias de segunda ordem do EMV em MLGHs}

Seja o logaritmo da função de verossimilhança para $\boldsymbol{\beta}$ e $\boldsymbol{\lambda}$ dado em (1.3). Utilizaremos os índices em minúsculos $r, s, t, \ldots$ para denotar as componentes do vetor $\boldsymbol{\beta}$ e os índices em maiúsculos $R, S, T, \ldots$ para as componentes do vetor $\boldsymbol{\lambda}$. Adotaremos a notação introduzida por Lawley (1956) para as derivadas do logaritmo da função de verossimilhança: $U_{r}=\partial \ell(\boldsymbol{\beta}, \boldsymbol{\lambda}) / \partial \beta_{r}, U_{r S}=\partial^{2} \ell(\boldsymbol{\beta}, \boldsymbol{\lambda}) / \partial \beta_{r} \partial \lambda_{S}, U_{r, S}=\partial \ell(\boldsymbol{\beta}, \boldsymbol{\lambda}) / \partial \beta_{r} \partial \ell(\beta, \lambda) / \partial \lambda_{S}$, $U_{r s T}=\partial^{3} \ell(\boldsymbol{\beta}, \boldsymbol{\lambda}) / \partial \beta_{r} \partial \beta_{s} \lambda_{T}$, etc. Os cumulantes conjuntos de $\ell(\boldsymbol{\beta}, \boldsymbol{\lambda})$ são $\kappa_{r S}=\mathrm{E}\left(U_{r S}\right)$, 
$\kappa_{r, S}=\mathrm{E}\left(U_{r, S}\right), \kappa_{r s T}=\mathrm{E}\left(U_{r s T}\right)$ e assim por diante. Definimos as derivadas dos cumulantes conjuntos por $\kappa_{r s}^{(t)}=\partial \kappa_{r s} / \partial \beta_{t}, \kappa_{r s}^{(T)}=\partial \kappa_{r s} / \partial \lambda_{T}, \kappa_{R S}^{(T U)}=\partial \kappa_{R S} / \partial \lambda_{T} \partial \lambda_{U}$, etc. Todos os $\kappa^{\prime} \mathrm{s}$ referem-se a um total sobre a amostra e são, em geral, de ordem $n$. Note que $\kappa_{r, s}=-\kappa_{r s}$ e $\kappa_{R, S}=-\kappa_{R S}$ são os elementos da matriz de informação esperada $\boldsymbol{K}_{\beta}$ e $\boldsymbol{K}_{\lambda}$, respectivamente. Os elementos correspondentes às inversas $\boldsymbol{K}_{\beta}^{-1}$ e $\boldsymbol{K}_{\lambda}^{-1}$ são $-\kappa^{r s}$ e $-\kappa^{R S}$.

Seja $\boldsymbol{\theta}=\left(\boldsymbol{\beta}^{\top}, \boldsymbol{\lambda}^{\top}\right)^{\top}$ o vetor de parâmetros desconhecidos no modelo (1.1) e (1.2) e $\hat{\boldsymbol{\theta}}$ o seu EMV. Com base nas expressões (2.1)-(2.5) podemos escrever a matriz de covariâncias de segunda ordem de $\hat{\boldsymbol{\theta}}$ como

$$
\operatorname{Cov}_{2}(\hat{\boldsymbol{\theta}})=\operatorname{Cov}_{1}(\hat{\boldsymbol{\theta}})+\Sigma,
$$

em que $\operatorname{Cov}_{1}(\hat{\boldsymbol{\theta}})$ é dada por

$$
\operatorname{Cov}_{1}(\hat{\boldsymbol{\theta}})=K^{-1}(\boldsymbol{\theta})=\left(\begin{array}{cc}
\boldsymbol{K}_{\beta}^{-1} & \mathbf{0} \\
\mathbf{0} & \boldsymbol{K}_{\lambda}^{-1}
\end{array}\right)
$$

$\operatorname{com} \boldsymbol{K}_{\beta}^{-1}=\left(\boldsymbol{X}^{\top} \boldsymbol{W} \boldsymbol{\Phi} \boldsymbol{X}\right)^{-1}$ e $\boldsymbol{K}_{\lambda}^{-1}=\left(-\boldsymbol{S}^{\top} \boldsymbol{D}_{2} \boldsymbol{\Phi}_{1}^{2} \boldsymbol{S}\right)^{-1}$ e a matriz $\boldsymbol{\Sigma}$ é definida como

$$
\Sigma=\left(\begin{array}{cc}
\Sigma_{\beta \beta} & \Sigma_{\beta \lambda} \\
\Sigma_{\beta \lambda}^{\top} & \Sigma_{\lambda \lambda}
\end{array}\right)
$$

$\operatorname{com} \Sigma_{\beta \beta}=\Sigma_{\beta \beta}^{(1)}+\Sigma_{\beta \beta}^{(2)}+\Sigma_{\beta \beta}^{(3)}, \Sigma_{\beta \lambda}=\Sigma_{\beta \lambda}^{(1)}+\Sigma_{\beta \lambda}^{(2)}+\Sigma_{\beta \lambda}^{(3)}$ e $\Sigma_{\lambda \lambda}=\Sigma_{\lambda \lambda}^{(1)}+\Sigma_{\lambda \lambda}^{(2)}+\Sigma_{\lambda \lambda}^{(3)}$ sendo $\Sigma_{\beta \beta}^{(r)}=\left\{\sigma_{i j}^{(r)}\right\}, \Sigma_{\beta \lambda}^{(r)}=\left\{\sigma_{i J}^{(r)}\right\}$ e $\Sigma_{\lambda \lambda}^{(r)}=\left\{\sigma_{I J}^{(r)}\right\}$ para $r=1,2$ e 3.

Definimos as seguintes matrizes:

$$
\begin{gathered}
\boldsymbol{P}_{\beta}=\left(\boldsymbol{X}^{\top} \boldsymbol{W} \boldsymbol{\Phi} \boldsymbol{X}\right)^{-1} \boldsymbol{X}^{\top}, \quad \boldsymbol{P}_{\lambda}=\left(-\boldsymbol{S}^{\top} \boldsymbol{D}_{2} \boldsymbol{\Phi}_{1}^{2} \boldsymbol{S}\right)^{-1} \boldsymbol{S}^{\top}, \\
\boldsymbol{Z}_{\beta}=\left\{z_{\beta \ell m}\right\}=\boldsymbol{X}\left(\boldsymbol{X}^{\top} \boldsymbol{W} \boldsymbol{\Phi} \boldsymbol{X}\right)^{-1} \boldsymbol{X}^{\top}, \quad \boldsymbol{Z}_{\lambda}=\left\{z_{\lambda \ell m}\right\}=\boldsymbol{S}\left(-\boldsymbol{S}^{\top} \boldsymbol{D}_{2} \boldsymbol{\Phi}_{1}^{2} \boldsymbol{S}\right)^{-1} \boldsymbol{S}^{\top}, \\
\boldsymbol{Z}_{\beta d}=\operatorname{diag}\left(z_{\beta 11}, \ldots, z_{\beta n n}\right), \quad \boldsymbol{Z}_{\lambda d}=\operatorname{diag}\left(z_{\lambda 11}, \ldots, z_{\lambda n n}\right), \\
\boldsymbol{F}=\operatorname{diag}\left\{f_{1}, \ldots, f_{n}\right\}, \quad \boldsymbol{G}=\operatorname{diag}\left\{g_{1}, \ldots, g_{n}\right\}, \quad \boldsymbol{H}=\operatorname{diag}\left\{h_{1}, \ldots, h_{n}\right\}, \\
\boldsymbol{\Phi}_{1}=\operatorname{diag}\left\{\phi_{11}, \ldots, \phi_{1 n}\right\}, \quad \boldsymbol{\Phi}_{2}=\operatorname{diag}\left\{\phi_{21}, \ldots, \phi_{2 n}\right\}, \quad \boldsymbol{\Phi}_{3}=\operatorname{diag}\left\{\phi_{31}, \ldots, \phi_{3 n}\right\}, \\
\boldsymbol{D}_{2}=\operatorname{diag}\left\{d_{21}, \ldots, d_{2 n}\right\}, \quad \boldsymbol{D}_{3}=\operatorname{diag}\left\{d_{31}, \ldots, d_{3 n}\right\} \quad \text { e } \quad \boldsymbol{D}_{4}=\operatorname{diag}\left\{d_{41}, \ldots, d_{4 n}\right\},
\end{gathered}
$$


com

$$
\begin{gathered}
f_{\ell}=\frac{1}{V_{\ell}} \frac{\mathrm{d} \mu_{\ell}}{\mathrm{d} \eta_{\ell}} \frac{\mathrm{d}^{2} \mu_{\ell}}{\mathrm{d} \eta_{\ell}^{2}}, \quad g_{\ell}=f_{\ell}-\frac{1}{V_{\ell}^{2}} \frac{\mathrm{d} V_{\ell}}{\mathrm{d} \mu_{\ell}}\left(\frac{\mathrm{d} \mu_{\ell}}{\mathrm{d} \eta_{\ell}}\right)^{3}, \\
h_{\ell}=\frac{1}{V_{\ell}^{3}}\left(\frac{\mathrm{d} V_{\ell}}{\mathrm{d} \mu_{\ell}}\right)^{2}\left(\frac{\mathrm{d} \mu_{\ell}}{\mathrm{d} \eta_{\ell}}\right)^{4}-\frac{1}{V_{\ell}}\left(\frac{\mathrm{d} \mu_{\ell}}{\mathrm{d} \eta_{\ell}}\right)\left(\frac{\mathrm{d}^{3} \mu_{\ell}}{\mathrm{d} \eta_{\ell}^{3}}\right)-\frac{1}{V_{\ell}^{2}}\left(\frac{\mathrm{d} V_{\ell}}{\mathrm{d} \mu_{\ell}}\right)\left(\frac{\mathrm{d} \mu_{\ell}}{\mathrm{d} \eta_{\ell}}\right)^{2}\left(\frac{\mathrm{d}^{2} \mu_{\ell}}{\mathrm{d} \eta_{\ell}^{2}}\right),
\end{gathered}
$$

$\phi_{k \ell}=\mathrm{d}^{k} \phi_{\ell} / \mathrm{d} \tau_{\ell}^{k}$ e $d_{k \ell}=\mathrm{d}^{k} d\left(\phi_{\ell}\right) / \mathrm{d} \phi_{\ell}^{k}$, para $k=1, \ldots, 4$ e $\ell=1, \ldots, n$. O produto direto entre matrizes será denotado pelo símbolo $\odot$.

Desenvolvendo as expressões (2.3)-(2.5) e após alguma álgebra, obtemos

$$
\begin{aligned}
& \boldsymbol{\Sigma}_{\beta \beta}^{(1)}=\boldsymbol{P}_{\beta} \boldsymbol{\Phi} \boldsymbol{H} \boldsymbol{Z}_{\beta d} \boldsymbol{P}_{\beta}^{\top}+\boldsymbol{P}_{\beta} \boldsymbol{\Phi}_{1}^{2} \boldsymbol{\Phi}^{-1} \boldsymbol{W} \boldsymbol{Z}_{\lambda d} \boldsymbol{P}_{\beta}^{\top}, \\
& \boldsymbol{\Sigma}_{\beta \beta}^{(2)}=\frac{3}{2} \boldsymbol{P}_{\beta} \boldsymbol{\Phi} \boldsymbol{F}\left(\boldsymbol{Z}_{\beta} \odot \boldsymbol{Z}_{\beta}\right) \boldsymbol{\Phi} \boldsymbol{F} \boldsymbol{P}_{\beta}^{\top}+\boldsymbol{P}_{\beta} \boldsymbol{\Phi} \boldsymbol{F}\left(\boldsymbol{Z}_{\beta} \odot \boldsymbol{Z}_{\beta}\right) \boldsymbol{\Phi} \boldsymbol{G} \boldsymbol{P}_{\beta}^{\top} \\
& -\boldsymbol{P}_{\beta} \boldsymbol{\Phi} \boldsymbol{G}\left(\boldsymbol{Z}_{\beta} \odot \boldsymbol{Z}_{\beta}\right) \boldsymbol{\Phi} \boldsymbol{G} \boldsymbol{P}_{\beta}^{\top}-\boldsymbol{P}_{\beta} \boldsymbol{\Phi}_{1} \boldsymbol{W}\left(\boldsymbol{Z}_{\beta} \odot \boldsymbol{Z}_{\lambda}\right) \boldsymbol{\Phi}_{1} \boldsymbol{W} \boldsymbol{P}_{\beta}^{\top}, \\
& \boldsymbol{\Sigma}_{\beta \beta}^{(3)}=\boldsymbol{P}_{\beta} \boldsymbol{\Phi}(\boldsymbol{F}+\boldsymbol{G}) \operatorname{diag}\left(\boldsymbol{Z}_{\beta} \boldsymbol{Z}_{\beta d} \boldsymbol{\Phi} \boldsymbol{F} \mathbf{1}\right) \boldsymbol{P}_{\beta}^{\top}, \\
& \boldsymbol{\Sigma}_{\beta \lambda}^{(1)}=\frac{3}{2} \boldsymbol{P}_{\beta} \boldsymbol{\Phi}_{1} \boldsymbol{G} \boldsymbol{Z}_{\beta d} \boldsymbol{P}_{\lambda}^{\top} \\
& \boldsymbol{\Sigma}_{\beta \lambda}^{(2)}=-\frac{1}{2} \boldsymbol{P}_{\beta} \boldsymbol{\Phi}(\boldsymbol{F}+3 \boldsymbol{G})\left(\boldsymbol{Z}_{\beta} \odot \boldsymbol{Z}_{\beta}\right) \boldsymbol{\Phi}_{1} \boldsymbol{W} \boldsymbol{P}_{\lambda}^{\top}, \\
& \boldsymbol{\Sigma}_{\beta \lambda}^{(3)}=\frac{1}{2} \boldsymbol{P}_{\beta} \boldsymbol{\Phi}_{1} \boldsymbol{W} \operatorname{diag}\left(\boldsymbol{Z}_{\beta} \boldsymbol{Z}_{\beta d} \boldsymbol{\Phi} \boldsymbol{F} \mathbf{1}\right) \boldsymbol{P}_{\lambda}^{\top} \\
& \boldsymbol{\Sigma}_{\lambda \lambda}^{(1)}=\boldsymbol{P}_{\lambda}\left(D_{4} \boldsymbol{\Phi}_{1}^{4}+4 \boldsymbol{D}_{3} \boldsymbol{\Phi}_{1}^{2} \boldsymbol{\Phi}_{2}+\boldsymbol{D}_{2} \boldsymbol{\Phi}_{1} \boldsymbol{\Phi}_{3}\right) Z_{\lambda d} \boldsymbol{P}_{\lambda}^{\top}+\boldsymbol{P}_{\lambda}\left(\boldsymbol{\Phi}_{2}+\boldsymbol{\Phi}_{1}^{2} \boldsymbol{\Phi}^{-1}\right) \boldsymbol{W} \boldsymbol{Z}_{\beta d} \boldsymbol{P}_{\lambda}^{\top}, \\
& \boldsymbol{\Sigma}_{\lambda \lambda}^{(2)}=\frac{3}{2} \boldsymbol{P}_{\lambda} \boldsymbol{D}_{3} \boldsymbol{\Phi}_{1}^{3}\left(\boldsymbol{Z}_{\lambda} \odot \boldsymbol{Z}_{\lambda}\right) \boldsymbol{D}_{3} \boldsymbol{\Phi}_{1}^{3} \boldsymbol{P}_{\lambda}^{\top}+2 \boldsymbol{P}_{\lambda} \boldsymbol{D}_{3} \boldsymbol{\Phi}_{1}^{3}\left(\boldsymbol{Z}_{\lambda} \odot \boldsymbol{Z}_{\lambda}\right) \boldsymbol{D}_{2} \boldsymbol{\Phi}_{1} \boldsymbol{\Phi}_{2} \boldsymbol{P}_{\lambda}^{\top} \\
& +2 \boldsymbol{P}_{\lambda} \boldsymbol{D}_{2} \boldsymbol{\Phi}_{1} \boldsymbol{\Phi}_{2}\left(\boldsymbol{Z}_{\lambda} \odot \boldsymbol{Z}_{\lambda}\right) \boldsymbol{D}_{3} \boldsymbol{\Phi}_{1}^{3} \boldsymbol{P}_{\lambda}^{\top}+\frac{3}{2} \boldsymbol{P}_{\lambda} \boldsymbol{D}_{2} \boldsymbol{\Phi}_{1} \boldsymbol{\Phi}_{2}\left(\boldsymbol{Z}_{\lambda} \odot \boldsymbol{Z}_{\lambda}\right) \boldsymbol{D}_{2} \boldsymbol{\Phi}_{1} \boldsymbol{\Phi}_{2} \boldsymbol{P}_{\lambda}^{\top} \\
& -\frac{3}{2} \boldsymbol{P}_{\lambda} \boldsymbol{\Phi}_{1} \boldsymbol{W}\left(\boldsymbol{Z}_{\beta} \odot \boldsymbol{Z}_{\beta}\right) \boldsymbol{\Phi}_{1} \boldsymbol{W} \boldsymbol{P}_{\lambda}^{\top}
\end{aligned}
$$




$$
\begin{aligned}
\boldsymbol{\Sigma}_{\lambda \lambda}^{(3)}= & \boldsymbol{P}_{\lambda}\left(\boldsymbol{D}_{3} \boldsymbol{\Phi}_{1}^{3}+2 \boldsymbol{D}_{2} \boldsymbol{\Phi}_{1} \boldsymbol{\Phi}_{2}\right) \operatorname{diag}\left[\boldsymbol{Z}_{\lambda} \boldsymbol{Z}_{\lambda d}\left(\boldsymbol{D}_{3} \boldsymbol{\Phi}_{1}^{3}+\boldsymbol{D}_{2} \boldsymbol{\Phi}_{1} \boldsymbol{\Phi}_{2}\right) \mathbf{1}\right] \boldsymbol{P}_{\lambda}^{\top} \\
& +\boldsymbol{P}_{\lambda}\left(\boldsymbol{D}_{3} \boldsymbol{\Phi}_{1}^{3}+2 \boldsymbol{D}_{2} \boldsymbol{\Phi}_{1} \boldsymbol{\Phi}_{2}\right) \operatorname{diag}\left(\boldsymbol{Z}_{\lambda} \boldsymbol{Z}_{\beta d} \boldsymbol{\Phi}_{1} \boldsymbol{W} \mathbf{1}\right) \boldsymbol{P}_{\lambda}^{\top}
\end{aligned}
$$

As expressões (2.9), (2.12), (2.13), (2.14) e (2.16) divergem das expressões (3.3), (3.4), (3.8), (3.9) e (3.10) obtidas por Barroso et al. (2013), respectivamente.

\subsubsection{Matriz de covariâncias de segunda ordem do EMV em MLGs}

Como os MLGHs são uma extensão dos MLGs, faremos agora as adaptações necessárias para obtermos as matrizes de covariâncias nos MLGs para os casos em que o parâmetro de dispersão é conhecido e desconhecido.

Consideremos inicialmente o parâmetro de dispersão desconhecido e o modelo definido em (1.1) e (1.2) com $t_{2}(\phi)$ agora sendo definido como $t_{2}(\phi)=\boldsymbol{\tau}=\lambda \mathbf{1}$, em que $\lambda$ é um escalar e 1 é um vetor $n \times 1$ de uns. Estabelecendo a ligação identidade para a função de ligação da dispersão temos $\boldsymbol{\phi}=\boldsymbol{\tau}=\lambda \mathbf{1}$ e isto implica que $\boldsymbol{\phi}=\left(\phi_{1}, \ldots, \phi_{n}\right)^{\top}=(\lambda, \ldots, \lambda)^{\top}$, ou seja, $\phi_{\ell}=\lambda$ para todo $\ell=1, \ldots, n$. Considere $\phi_{\ell}=\phi$ para todo $\ell=1, \ldots, n$, segue portanto que $\phi=\lambda$. Diante destes fatos as matrizes definidas na Seção 2.1.1 são dadas por:

$$
\begin{gathered}
\boldsymbol{\Phi}=\phi \boldsymbol{I}_{n}, \quad \boldsymbol{\Phi}_{1}=\boldsymbol{I}_{n}, \quad \boldsymbol{\Phi}_{2}=\boldsymbol{\Phi}_{3}=\mathbf{0}_{n}, \\
\boldsymbol{D}_{2}=d_{2} \boldsymbol{I}_{n}, \quad \boldsymbol{D}_{3}=d_{3} \boldsymbol{I}_{n}, \quad \boldsymbol{D}_{4}=d_{4} \boldsymbol{I}_{n}, \\
K_{\lambda}=K_{\phi}=-n d_{2}, \quad \boldsymbol{P}_{\lambda}=\boldsymbol{P}_{\phi}=-\frac{1}{n d_{2}} \mathbf{1}^{\top}, \\
\boldsymbol{Z}_{\lambda}=\boldsymbol{Z}_{\phi}=-\frac{1}{n d_{2}} \mathbf{1 1}^{\top}, \quad \boldsymbol{Z}_{\lambda_{d}}=\boldsymbol{Z}_{\phi_{d}}=-\frac{1}{n d_{2}} \boldsymbol{I}_{n}, \\
\boldsymbol{K}_{\beta}=\phi \boldsymbol{X}^{\top} \boldsymbol{W} \boldsymbol{X}, \quad \boldsymbol{P}_{\beta}=\phi^{-1}\left(\boldsymbol{X}^{\top} \boldsymbol{W} \boldsymbol{X}^{-1} \boldsymbol{X}^{\top}=\phi^{-1} \boldsymbol{P},\right. \\
\boldsymbol{Z}_{\beta}=\phi^{-1} \boldsymbol{X}\left(\boldsymbol{X}^{\top} \boldsymbol{W} \boldsymbol{X}\right)^{-1} \boldsymbol{X}^{\top}=\phi^{-1} \boldsymbol{Z} \quad \mathrm{e} \quad \boldsymbol{Z}_{\beta d}=\phi^{-1} \operatorname{diag}\left(z_{11}, \ldots, z_{n n}\right)=\phi^{-1} \boldsymbol{Z}_{d} .
\end{gathered}
$$

Utilizando as matrizes definidas em (2.18)-(2.23) nas equações (2.9)-(2.17), obtemos

$$
\boldsymbol{\Sigma}_{\beta \beta}^{(1)}=\frac{1}{\phi^{2}} \boldsymbol{P} \boldsymbol{H} \boldsymbol{Z}_{d} \boldsymbol{P}^{\top}-\frac{1}{n d_{2} \phi^{3}} \boldsymbol{P} \boldsymbol{W} \boldsymbol{P}^{\top}
$$




$$
\begin{aligned}
& \boldsymbol{\Sigma}_{\beta \beta}^{(2)}=\frac{3}{2 \phi^{2}} \boldsymbol{P} \boldsymbol{F}(\boldsymbol{Z} \odot \boldsymbol{Z}) \boldsymbol{F} \boldsymbol{P}^{\top}+\frac{1}{\phi^{2}} \boldsymbol{P} \boldsymbol{F}(\boldsymbol{Z} \odot \boldsymbol{Z}) \boldsymbol{G} \boldsymbol{P}^{\top} \\
& -\frac{1}{\phi^{2}} \boldsymbol{P} \boldsymbol{G}(\boldsymbol{Z} \odot \boldsymbol{Z}) \boldsymbol{G} \boldsymbol{P}^{\top}+\frac{1}{n d_{2} \phi^{3}} \boldsymbol{P} \boldsymbol{W} \boldsymbol{Z} \boldsymbol{W} \boldsymbol{P}^{\top}, \\
& \boldsymbol{\Sigma}_{\beta \beta}^{(3)}=\frac{1}{\phi^{2}} \boldsymbol{P}(\boldsymbol{F}+\boldsymbol{G}) \operatorname{diag}\left(\boldsymbol{Z} \boldsymbol{Z}_{d} \boldsymbol{F} \mathbf{1}\right) \boldsymbol{P}^{\top}, \\
& \boldsymbol{\Sigma}_{\beta \phi}^{(1)}=-\frac{3}{2 n d_{2} \phi^{2}} \boldsymbol{P} \boldsymbol{G} \boldsymbol{Z}_{d} \mathbf{1}, \\
& \boldsymbol{\Sigma}_{\beta \phi}^{(2)}=\frac{1}{2 n d_{2} \phi^{2}} \boldsymbol{P}(\boldsymbol{F}+3 \boldsymbol{G})(\boldsymbol{Z} \odot \boldsymbol{Z}) \boldsymbol{W} \mathbf{1}, \\
& \boldsymbol{\Sigma}_{\beta \phi}^{(3)}=-\frac{1}{2 n d_{2} \phi^{2}} \boldsymbol{P} \boldsymbol{W} \boldsymbol{Z} \boldsymbol{Z}_{d} \boldsymbol{F} \mathbf{1}, \\
& \Sigma_{\phi \phi}^{(1)}=-\frac{d_{4}}{n^{2} d_{2}^{3}}+\frac{p}{n^{2} d_{2}^{2} \phi^{2}}, \\
& \Sigma_{\phi \phi}^{(2)}=\frac{3 d_{3}^{2}}{2 n^{2} d_{2}^{4}}-\frac{3}{2 n^{2} d_{2}^{2} \phi^{2}} \mathbf{1}^{\top} \boldsymbol{W}(\boldsymbol{Z} \odot \boldsymbol{Z}) \boldsymbol{W} \mathbf{1}
\end{aligned}
$$

e

$$
\Sigma_{\phi \phi}^{(3)}=\frac{d_{3}^{2}}{n^{2} d_{2}^{4}}-\frac{p d_{3}}{n^{2} d_{2}^{3} \phi} .
$$

As expressões (2.26), (2.27), (2.28) e (2.29) diferem das expressões obtidas por Cordeiro et al. (2006), a saber (3.6), (3.2), (3.7) e (3.8), respectivamente.

Considerando agora o parâmetro de dispersão conhecido, o resultado obtido foi o mesmo que Barroso et al. $(2013)^{1}$, a saber

$$
\begin{gathered}
\boldsymbol{\Sigma}_{\beta \beta}^{(1)}=\frac{1}{\phi^{2}} \boldsymbol{P} \boldsymbol{H} \boldsymbol{Z}_{d} \boldsymbol{P}^{\top} \\
\boldsymbol{\Sigma}_{\beta \beta}^{(2)}=\frac{3}{2 \phi^{2}} \boldsymbol{P} \boldsymbol{F}(\boldsymbol{Z} \odot \boldsymbol{Z}) \boldsymbol{F} \boldsymbol{P}^{\top}+\frac{1}{\phi^{2}} \boldsymbol{P} \boldsymbol{F}(\boldsymbol{Z} \odot \boldsymbol{Z}) \boldsymbol{G} \boldsymbol{P}^{\top} \\
-\frac{1}{\phi^{2}} \boldsymbol{P} \boldsymbol{G}(\boldsymbol{Z} \odot \boldsymbol{Z}) \boldsymbol{G} \boldsymbol{P}^{\top}
\end{gathered}
$$

\footnotetext{
${ }^{1}$ Corrigiu o resultado de Cordeiro (2004).
} 


$$
\boldsymbol{\Sigma}_{\beta \beta}^{(3)}=\frac{1}{\phi^{2}} \boldsymbol{P}(\boldsymbol{F}+\boldsymbol{G}) \operatorname{diag}\left(\boldsymbol{Z} \boldsymbol{Z}_{d} \boldsymbol{F} \mathbf{1}\right) \boldsymbol{P}^{\top}
$$

Vale ressaltar que para o MLG com parâmetro de dispersão conhecido, tanto as expressões de Shenton e Bowman (1977, p. 67) quanto as de Peers e Iqbal (1985) levaram ao mesmo resultado para a matriz de covariâncias de segunda ordem.

\subsection{Matriz de covariâncias de segunda ordem dos esti- madores corrigidos}

Seja $\overline{\boldsymbol{\theta}}=\left(\overline{\boldsymbol{\beta}}^{\top}, \overline{\boldsymbol{\lambda}}^{\top}\right)^{\top}$ o EMV corrigido pelo viés de ordem $n^{-1}$ em que $\overline{\boldsymbol{\beta}}=\hat{\boldsymbol{\beta}}-\hat{\boldsymbol{B}}(\hat{\boldsymbol{\beta}})$ e $\overline{\boldsymbol{\lambda}}=\hat{\boldsymbol{\lambda}}-\hat{\boldsymbol{B}}(\hat{\boldsymbol{\lambda}})$, sendo $\hat{\boldsymbol{B}}(\hat{\boldsymbol{\beta}})$ e $\hat{\boldsymbol{B}}(\hat{\boldsymbol{\lambda}})$ os vieses de ordem $n^{-1}$ de $\hat{\boldsymbol{\beta}}$ e $\hat{\boldsymbol{\lambda}}$, respectivamente, avaliados em $\left(\hat{\boldsymbol{\beta}}^{\top}, \hat{\boldsymbol{\lambda}}^{\top}\right)^{\top}$. As expressões matriciais para $\boldsymbol{B}(\boldsymbol{\beta})$ e $\boldsymbol{B}(\boldsymbol{\lambda})$ foram obtidas por Botter e Cordeiro (1998).

De Cordeiro et al. (2014) podemos escrever a matriz de covariâncias até a ordem $n^{-2}$ de $\overline{\boldsymbol{\theta}}=\left(\overline{\boldsymbol{\beta}}^{\top}, \overline{\boldsymbol{\lambda}}^{\top}\right)^{\top}$ como

$$
\operatorname{Cov}_{2}(\overline{\boldsymbol{\theta}})=\operatorname{Cov}_{2}(\hat{\boldsymbol{\theta}})+\boldsymbol{\Delta}+\boldsymbol{\Delta}^{\top}
$$

em que a $\operatorname{Cov}_{2}(\hat{\boldsymbol{\theta}})$ é dada em $(2.6)$ e o $(a, b)$-ésimo elemento de $\boldsymbol{\Delta}$ é $\delta_{a b} \operatorname{com} \delta_{a b}=\delta_{a b}^{(1)}+\delta_{a b}^{(2)}$ sendo

$$
\delta_{a b}^{(1)}=-\sum_{\beta, \lambda} \kappa^{a v} \kappa^{b w} \kappa^{s y} \kappa^{t u}\left(\kappa_{s t u}+2 \kappa_{s t, u}\right)\left(\kappa_{v w y}+\kappa_{v, w y}\right)
$$

e

$$
\delta_{a b}^{(2)}=\sum_{\beta, \lambda} \frac{1}{2} \kappa^{a v} \kappa^{b s} \kappa^{t u}\left[\kappa_{s t u v}+\kappa_{s t u, v}+2 \kappa_{s t v, u}+2\left(\kappa_{s t, u v}+\kappa_{s t, u, v}\right)\right] .
$$

A matriz $\Delta$ pode ser expressa como

$$
\Delta=\left(\begin{array}{cc}
\Delta_{\beta \beta} & \Delta_{\beta \lambda} \\
\Delta_{\lambda \beta} & \Delta_{\lambda \lambda}
\end{array}\right)
$$

em que $\boldsymbol{\Delta}_{\beta \beta}=\boldsymbol{\Delta}_{\beta \beta}^{(1)}+\boldsymbol{\Delta}_{\beta \beta}^{(2)}$ é uma matriz de dimensão $p \times p, \boldsymbol{\Delta}_{\beta \lambda}=\boldsymbol{\Delta}_{\beta \lambda}^{(1)}+\boldsymbol{\Delta}_{\beta \lambda}^{(2)}$ é uma matriz de dimensão $p \times q, \Delta_{\lambda \beta}=\Delta_{\lambda \beta}^{(1)}+\Delta_{\lambda \beta}^{(2)}$ é uma matriz de dimensão $q \times p$ e $\boldsymbol{\Delta}_{\lambda \lambda}=\boldsymbol{\Delta}_{\lambda \lambda}^{(1)}+\boldsymbol{\Delta}_{\lambda \lambda}^{(2)}$ é uma matriz de dimensão $q \times q$, sendo $\boldsymbol{\Delta}_{\beta \beta}^{(r)}=\left\{\delta_{a b}^{(r)}\right\}, \boldsymbol{\Delta}_{\beta \lambda}^{(r)}=\left\{\delta_{a B}^{(r)}\right\}, \boldsymbol{\Delta}_{\lambda \beta}^{(r)}=\left\{\delta_{A b}^{(r)}\right\}$ e $\boldsymbol{\Delta}_{\lambda \lambda}^{(r)}=\left\{\delta_{A B}^{(r)}\right\}$ para $r=1$ e 2. 
Desenvolvendo as expressões (2.34) e (2.35), obtemos

$$
\begin{aligned}
& \delta_{a b}^{(1)}=-\sum_{\beta} \kappa^{a v} \kappa^{b w} \kappa^{s y} \kappa^{t u}\left(\kappa_{s t u}+2 \kappa_{s t, u}\right)\left(\kappa_{v w y}+\kappa_{v, w y}\right) \\
& -\sum_{\beta, \lambda} \kappa^{a v} \kappa^{b w} \kappa^{s y} \kappa^{T U}\left(\kappa_{s T U}+2 \kappa_{s T, U}\right)\left(\kappa_{v w y}+\kappa_{v, w y}\right) \\
& -\sum_{\beta, \lambda} \kappa^{a v} \kappa^{b w} \kappa^{S Y} \kappa^{t u}\left(\kappa_{S t u}+2 \kappa_{S t, u}\right)\left(\kappa_{v w Y}+\kappa_{v, w Y}\right) \\
& -\sum_{\beta, \lambda} \kappa^{a v} \kappa^{b w} \kappa^{S Y} \kappa^{T U}\left(\kappa_{S T U}+2 \kappa_{S T, U}\right)\left(\kappa_{v w Y}+\kappa_{v, w Y}\right), \\
& \delta_{a b}^{(2)}=\frac{1}{2} \sum_{\beta} \kappa^{a v} \kappa^{b s} \kappa^{t u}\left[\kappa_{s t u v}+\kappa_{s t u, v}+2 \kappa_{s t v, u}+2\left(\kappa_{s t, u v}+\kappa_{s t, u, v}\right)\right] \\
& +\frac{1}{2} \sum_{\beta, \lambda} \kappa^{a v} \kappa^{b s} \kappa^{T U}\left[\kappa_{s T U v}+\kappa_{s T U, v}+2 \kappa_{s T v, U}+2\left(\kappa_{s T, U v}+\kappa_{s T, U, v}\right)\right], \\
& \delta_{a B}^{(1)}=-\sum_{\beta, \lambda} \kappa^{a v} \kappa^{B W} \kappa^{s y} \kappa^{t u}\left(\kappa_{s t u}+2 \kappa_{s t, u}\right)\left(\kappa_{v W y}+\kappa_{v, W y}\right) \\
& -\sum_{\beta, \lambda} \kappa^{a v} \kappa^{B W} \kappa^{s y} \kappa^{T U}\left(\kappa_{s T U}+2 \kappa_{s T, U}\right)\left(\kappa_{v W y}+\kappa_{v, W y}\right) \\
& -\sum_{\beta, \lambda} \kappa^{a v} \kappa^{B W} \kappa^{S Y} \kappa^{t u}\left(\kappa_{S t u}+2 \kappa_{S t, u}\right)\left(\kappa_{v W Y}+\kappa_{v, W Y}\right) \\
& -\sum_{\beta, \lambda} \kappa^{a v} \kappa^{B W} \kappa^{S Y} \kappa^{T U}\left(\kappa_{S T U}+2 \kappa_{S T, U}\right)\left(\kappa_{v W Y}+\kappa_{v, W Y}\right), \\
& \delta_{a B}^{(2)}=\frac{1}{2} \sum_{\beta, \lambda} \kappa^{a v} \kappa^{B S} \kappa^{t u}\left[\kappa_{S t u v}+\kappa_{S t u, v}+2 \kappa_{S t v, u}+2\left(\kappa_{S t, u v}+\kappa_{S t, u, v}\right)\right] \\
& +\frac{1}{2} \sum_{\beta, \lambda} \kappa^{a v} \kappa^{B S} \kappa^{T U}\left[\kappa_{S T U v}+\kappa_{S T U, v}+2 \kappa_{S T v, U}+2\left(\kappa_{S T, U v}+\kappa_{S T, U, v}\right)\right], \\
& \delta_{A b}^{(1)}=-\sum_{\beta, \lambda} \kappa^{A V} \kappa^{b w} \kappa^{s y} \kappa^{t u}\left(\kappa_{s t u}+2 \kappa_{s t, u}\right)\left(\kappa_{V w y}+\kappa_{V, w y}\right) \\
& -\sum_{\beta, \lambda} \kappa^{A V} \kappa^{b w} \kappa^{s y} \kappa^{T U}\left(\kappa_{s T U}+2 \kappa_{s T, U}\right)\left(\kappa_{V w y}+\kappa_{V, w y}\right) \\
& -\sum_{\beta, \lambda} \kappa^{A V} \kappa^{b w} \kappa^{S Y} \kappa^{t u}\left(\kappa_{S t u}+2 \kappa_{S t, u}\right)\left(\kappa_{V w Y}+\kappa_{V, w Y}\right) \\
& -\sum_{\beta, \lambda} \kappa^{A V} \kappa^{b w} \kappa^{S Y} \kappa^{T U}\left(\kappa_{S T U}+2 \kappa_{S T, U}\right)\left(\kappa_{V w Y}+\kappa_{V, w Y}\right)
\end{aligned}
$$




$$
\begin{aligned}
\delta_{A b}^{(2)}= & \frac{1}{2} \sum_{\beta, \lambda} \kappa^{A V} \kappa^{b s} \kappa^{t u}\left[\kappa_{s t u V}+\kappa_{s t u, V}+2 \kappa_{s t V, u}+2\left(\kappa_{s t, u V}+\kappa_{s t, u, V}\right)\right] \\
+ & \frac{1}{2} \sum_{\beta, \lambda} \kappa^{A V} \kappa^{b s} \kappa^{T U}\left[\kappa_{s T U V}+\kappa_{s T U, V}+2 \kappa_{s T V, U}+2\left(\kappa_{s T, U V}+\kappa_{s T, U, V}\right)\right] \\
\delta_{A B}^{(1)}= & -\sum_{\lambda} \kappa^{A V} \kappa^{B W} \kappa^{S Y} \kappa^{T U}\left(\kappa_{S T U}+2 \kappa_{S T, U}\right)\left(\kappa_{V W Y}+\kappa_{V, W Y}\right) \\
& -\sum_{\beta, \lambda} \kappa^{A V} \kappa^{B W} \kappa^{S Y} \kappa^{t u}\left(\kappa_{S t u}+2 \kappa_{S t, u}\right)\left(\kappa_{V W Y}+\kappa_{V, W Y}\right) \\
& -\sum_{\beta, \lambda} \kappa^{A V} \kappa^{B W} \kappa^{s y} \kappa^{T U}\left(\kappa_{s T U}+2 \kappa_{s T, U}\right)\left(\kappa_{V W y}+\kappa_{V, W y}\right) \\
& -\sum_{\beta, \lambda} \kappa^{A V} \kappa^{B W} \kappa^{s y} \kappa^{t u}\left(\kappa_{s t u}+2 \kappa_{s t, u}\right)\left(\kappa_{V W y}+\kappa_{V, W y}\right)
\end{aligned}
$$

e

$$
\begin{aligned}
\delta_{A B}^{(2)}= & \frac{1}{2} \sum_{\lambda} \kappa^{A V} \kappa^{B S} \kappa^{T U}\left[\kappa_{S T U V}+\kappa_{S T U, V}+2 \kappa_{S T V, U}+2\left(\kappa_{S T, U V}+\kappa_{S T, U, V}\right)\right] \\
& +\frac{1}{2} \sum_{\beta, \lambda} \kappa^{A V} \kappa^{B S} \kappa^{t u}\left[\kappa_{S t u V}+\kappa_{S t u, V}+2 \kappa_{S t V, u}+2\left(\kappa_{S t, u V}+\kappa_{S t, u, V}\right)\right] .
\end{aligned}
$$

Utilizando os cumulantes apresentados no Apêndice A e após alguma álgebra temos que as expressões (2.37)-(2.44) resultam em

$$
\begin{aligned}
& \delta_{a b}^{(1)}=-\sum_{\ell, m=1}^{n}\{\left(\sum_{v=1}^{p} \kappa^{a v} x_{v \ell}\right)\left[-\phi_{\ell}\left(f_{\ell}+g_{\ell}\right)\right]\left(\sum_{s, y=1}^{p} x_{\ell y} \kappa^{y s} x_{s m}\right) \\
&\left.\times\left(\sum_{t, u=1}^{p} x_{m t} \kappa^{t u} x_{u m}\right)\left(-\phi_{m} f_{m}\right)\left(\sum_{w=1}^{p} x_{\ell w} \kappa^{w b}\right)\right\}, \\
& \delta_{a b}^{(2)}=\frac{1}{2} \sum_{\ell=1}^{n}\left\{\left(\sum_{v=1}^{p} \kappa^{a v} x_{v \ell}\right)\left(\phi_{\ell} d_{\ell}\right)\left(\sum_{t, u=1}^{p} x_{\ell t} \kappa^{t u} x_{u \ell}\right)\left(\sum_{s=1}^{p} x_{\ell s} \kappa^{s b}\right)\right\},
\end{aligned}
$$

em que $d_{\ell}=V_{\ell}^{-2}\left(\mathrm{~d} V_{\ell} / \mathrm{d} \mu_{\ell}\right)\left(\mathrm{d} \mu_{\ell} / \mathrm{d} \eta_{\ell}\right)^{2}\left(\mathrm{~d}^{2} \mu_{\ell} / \mathrm{d} \eta_{\ell}^{2}\right)-V_{\ell}^{-1}\left(\mathrm{~d} \mu_{\ell} / \mathrm{d} \eta_{\ell}\right)\left(\mathrm{d}^{3} \mu_{\ell} / \mathrm{d} \eta_{\ell}^{3}\right)-V_{\ell}^{-1}\left(\mathrm{~d}^{2} \mu_{\ell} / \mathrm{d} \eta_{\ell}^{2}\right)^{2}$,

$$
\begin{gathered}
\delta_{a B}^{(1)}=0, \\
\delta_{a B}^{(2)}=\frac{1}{2} \sum_{\ell=1}^{n}\left\{\left(\sum_{v=1}^{p} \kappa^{a v} x_{v \ell}\right)\left[\phi_{1 \ell}\left(f_{\ell}+g_{\ell}\right)\right]\left(\sum_{t, u=1}^{p} x_{\ell t} \kappa^{t u} x_{u \ell}\right)\left(\sum_{S=1}^{q} s_{\ell S} \kappa^{S B}\right)\right\},
\end{gathered}
$$




$$
\begin{gathered}
\delta_{A b}^{(1)}=-\sum_{\ell, m=1}^{n}\left\{\left(\sum_{V=1}^{q} \kappa^{A V} s_{V \ell}\right)\left(-\phi_{1 \ell} w_{\ell}\right)\left(\sum_{s, y=1}^{p} x_{\ell y} \kappa^{y s} x_{s m}\right)\right. \\
\left.\times\left(\sum_{t, u=1}^{p} x_{m t} \kappa^{t u} x_{u m}\right)\left(-\phi_{m} f_{m}\right)\left(\sum_{w=1}^{p} x_{\ell w} \kappa^{w b}\right)\right\} \\
\delta_{A b}^{(2)}=-\frac{1}{2} \sum_{\ell=1}^{n}\left\{\left(\sum_{V=1}^{q} \kappa^{A V} s_{V \ell}\right)\left(\phi_{1 \ell} f_{\ell}\right)\left(\sum_{t, u=1}^{p} x_{\ell t} \kappa^{t u} x_{u \ell}\right)\left(\sum_{s=1}^{p} x_{\ell s} \kappa^{s b}\right)\right\} \\
\delta_{A B}^{(1)}=-\sum_{\ell, m=1}^{n}\left\{\left(\sum_{V=1}^{q} \kappa^{A V} s_{V \ell}\right)\left(d_{3 \ell} \phi_{1 \ell}^{3}+2 d_{2 \ell} \phi_{1 \ell} \phi_{2 \ell}\right)\left(\sum_{S, Y=1}^{q} s_{\ell Y} \kappa^{Y S} s_{S m}\right)\right. \\
\left.\times\left(\sum_{T, U=1}^{q} s_{m T} \kappa^{T U} s_{U m}\right)\left(d_{3 m} \phi_{1 m}^{3}+d_{2 m} \phi_{1 m} \phi_{2 m}\right)\left(\sum_{W=1}^{q} s_{\ell W} \kappa^{W B}\right)\right\} \\
-\sum_{\ell, m=1}^{n}\left\{\left(\sum_{V=1}^{q} \kappa^{A V} s_{V \ell}\right)\left(d_{3 \ell} \phi_{1 \ell}^{3}+2 d_{2 \ell} \phi_{1 \ell} \phi_{2 \ell}\right)\left(\sum_{S, Y=1}^{q} s_{\ell Y} \kappa^{Y S} s_{S m}\right)\right. \\
\\
\left.\times\left(\sum_{t, u=1}^{p} x_{m t} \kappa^{t u} x_{u m}\right)\left(\phi_{1 m} w_{m}\right)\left(\sum_{W=1}^{q} s_{\ell W} \kappa^{W B}\right)\right\}
\end{gathered}
$$

e

$$
\begin{aligned}
\delta_{A B}^{(2)}= & \frac{1}{2} \sum_{\ell=1}^{n}\left\{\left(\sum_{V=1}^{q} \kappa^{A V} s_{V \ell}\right)\left(d_{4 \ell} \phi_{1 \ell}^{4}+4 d_{3 \ell} \phi_{1 \ell}^{2} \phi_{2 \ell}+d_{2 \ell} \phi_{2 \ell}^{2}+d_{2 \ell} \phi_{1 \ell} \phi_{3 \ell}\right)\right. \\
& \left.\times\left(\sum_{T, U=1}^{q} s_{\ell T} \kappa^{T U} s_{U \ell}\right)\left(\sum_{S=1}^{q} s_{\ell S} \kappa^{S B}\right)\right\} \\
& +\frac{1}{2} \sum_{\ell=1}^{n}\left\{\left(\sum_{V=1}^{q} \kappa^{A V} s_{V \ell}\right)\left(\phi_{2 \ell} w_{\ell}\right)\left(\sum_{t, u=1}^{p} x_{\ell t} \kappa^{t u} x_{u \ell}\right)\left(\sum_{S=1}^{q} s_{\ell S} \kappa^{S B}\right)\right\} .
\end{aligned}
$$

As expressões (2.45)-(2.52) podem ser escritas em notação matricial como

$$
\begin{gathered}
\boldsymbol{\Delta}_{\beta \beta}^{(1)}=-\boldsymbol{P}_{\beta} \boldsymbol{\Phi}(\boldsymbol{F}+\boldsymbol{G}) \operatorname{diag}\left(\boldsymbol{Z}_{\beta} \boldsymbol{Z}_{\beta d} \boldsymbol{\Phi} \boldsymbol{F} \mathbf{1}\right) \boldsymbol{P}_{\beta}^{\top}, \\
\boldsymbol{\Delta}_{\beta \beta}^{(2)}=-\frac{1}{2} \boldsymbol{P}_{\beta} \boldsymbol{\Phi} \boldsymbol{D} \boldsymbol{Z}_{\beta d} \boldsymbol{P}_{\beta}^{\top},
\end{gathered}
$$

em que $\boldsymbol{D}=\operatorname{diag}\left\{d_{1}, \ldots, d_{n}\right\}$,

$$
\boldsymbol{\Delta}_{\beta \lambda}^{(1)}=\mathbf{0},
$$




$$
\begin{gathered}
\boldsymbol{\Delta}_{\beta \lambda}^{(2)}=-\frac{1}{2} \boldsymbol{P}_{\beta} \boldsymbol{\Phi}_{1}(\boldsymbol{F}+\boldsymbol{G}) \boldsymbol{Z}_{\beta d} \boldsymbol{P}_{\lambda}^{\top} \\
\boldsymbol{\Delta}_{\lambda \beta}^{(1)}=-\boldsymbol{P}_{\lambda} \boldsymbol{\Phi}_{1} \boldsymbol{W} \operatorname{diag}\left(\boldsymbol{Z}_{\beta} \boldsymbol{Z}_{\beta d} \boldsymbol{\Phi} \boldsymbol{F} \mathbf{1}\right) \boldsymbol{P}_{\beta}^{\top} \\
\boldsymbol{\Delta}_{\lambda \beta}^{(2)}=\frac{1}{2} \boldsymbol{P}_{\lambda} \boldsymbol{\Phi}_{1} \boldsymbol{F} \boldsymbol{Z}_{\beta d} \boldsymbol{P}_{\beta}^{\top}, \\
\boldsymbol{\Delta}_{\lambda \lambda}^{(1)}=-\boldsymbol{P}_{\lambda}\left(\boldsymbol{D}_{3} \boldsymbol{\Phi}_{1}^{3}+2 \boldsymbol{D}_{2} \boldsymbol{\Phi}_{1} \boldsymbol{\Phi}_{2}\right) \operatorname{diag}\left[\boldsymbol{Z}_{\lambda} \boldsymbol{Z}_{\lambda d}\left(\boldsymbol{D}_{3} \boldsymbol{\Phi}_{1}^{3}+\boldsymbol{D}_{2} \boldsymbol{\Phi}_{1} \boldsymbol{\Phi}_{2}\right) \mathbf{1}\right] \boldsymbol{P}_{\lambda}^{\top} \\
-\boldsymbol{P}_{\lambda}\left(\boldsymbol{D}_{3} \boldsymbol{\Phi}_{1}^{3}+2 \boldsymbol{D}_{2} \boldsymbol{\Phi}_{1} \boldsymbol{\Phi}_{2}\right) \operatorname{diag}\left(\boldsymbol{Z}_{\lambda} \boldsymbol{Z}_{\beta d} \boldsymbol{\Phi}_{1} \boldsymbol{W} \mathbf{1}\right) \boldsymbol{P}_{\lambda}^{\top}
\end{gathered}
$$

e

$$
\begin{aligned}
\boldsymbol{\Delta}_{\lambda \lambda}^{(2)}= & -\frac{1}{2} \boldsymbol{P}_{\lambda}\left(\boldsymbol{D}_{4} \boldsymbol{\Phi}_{1}^{4}+4 \boldsymbol{D}_{3} \boldsymbol{\Phi}_{1}^{2} \boldsymbol{\Phi}_{2}+\boldsymbol{D}_{2} \boldsymbol{\Phi}_{2}^{2}+\boldsymbol{D}_{2} \boldsymbol{\Phi}_{1} \boldsymbol{\Phi}_{3}\right) \boldsymbol{Z}_{\lambda d} \boldsymbol{P}_{\lambda}^{\top} \\
& -\frac{1}{2} \boldsymbol{P}_{\lambda} \boldsymbol{\Phi}_{2} \boldsymbol{W} \boldsymbol{Z}_{\beta d} \boldsymbol{P}_{\lambda}^{\top} .
\end{aligned}
$$

Segue portanto que as expressões (2.33), (2.36), (2.53)-(2.60) são os resultados desta seção.

\subsection{Casos particulares}

Os casos particulares da matriz de covariâncias de segunda ordem dos EMVs corrigidos pelo viés de primeira ordem nos MLGHs ocorrem quando consideramos os MLGs com dispersão conhecida e desconhecida. Para o caso em que a dispersão é conhecida, o resultado foi obtido por Cordeiro et al. (2014). Apresentaremos aqui as expressões para o caso em que a dispersão é desconhecida.

Consideremos o mesmo contexto utilizado na Subseção 2.1.2 em que o modelo é definido por (1.1) e (1.2) com $t_{2}(\phi)$ agora sendo definido como $t_{2}(\phi)=\boldsymbol{\tau}=\lambda \mathbf{1}$, em que $\lambda$ é um escalar e 1 é um vetor $n \times 1$ de uns. Estabelecendo a ligação identidade para a função de ligação da dispersão temos $\boldsymbol{\phi}=\boldsymbol{\tau}=\lambda \mathbf{1}$ e isto implica que $\boldsymbol{\phi}=\left(\phi_{1}, \ldots, \phi_{n}\right)^{\top}=(\lambda, \ldots, \lambda)^{\top}$, ou seja, $\phi_{\ell}=\lambda$ para todo $\ell=1, \ldots, n$. Considere $\phi_{\ell}=\phi$ para todo $\ell=1, \ldots, n$, segue portanto que $\lambda=\phi$.

Utilizando as matrizes definidas em (2.18)-(2.23) nas expressões (2.53)-(2.60), obtemos

$$
\boldsymbol{\Delta}_{\beta \beta}^{(1)}=-\frac{1}{\phi^{2}} \boldsymbol{P}(\boldsymbol{F}+\boldsymbol{G}) \operatorname{diag}\left(\boldsymbol{Z} \boldsymbol{Z}_{d} \boldsymbol{F} \mathbf{1}\right) \boldsymbol{P}^{\top},
$$




$$
\begin{gathered}
\boldsymbol{\Delta}_{\beta \beta}^{(2)}=-\frac{1}{2 \phi^{2}} \boldsymbol{P} \boldsymbol{D} \boldsymbol{Z}_{d} \boldsymbol{P}^{\top}, \\
\boldsymbol{\Delta}_{\beta \phi}^{(1)}=\mathbf{0}, \\
\boldsymbol{\Delta}_{\beta \phi}^{(2)}=\frac{1}{2 n d_{2} \phi^{2}} \boldsymbol{P}(\boldsymbol{F}+\boldsymbol{G}) \boldsymbol{Z}_{d} \mathbf{1}, \\
\boldsymbol{\Delta}_{\phi \beta}^{(1)}=-\frac{1}{n d_{2} \phi^{2}}\left(\boldsymbol{Z} \boldsymbol{Z}_{d} \boldsymbol{F} \mathbf{1}\right)^{\top} \boldsymbol{W} \boldsymbol{P}^{\top}, \\
\boldsymbol{\Delta}_{\phi \beta}^{(2)}=-\frac{1}{2 n d_{2} \phi^{2}} \mathbf{1}^{\top} \boldsymbol{F} \boldsymbol{Z}_{d} \boldsymbol{P}^{\top}, \\
\Delta_{\phi \phi}^{(1)}=-\frac{d_{3}^{2}}{n^{2} d_{2}^{4}}+\frac{p d_{3}}{n^{2} d_{2}^{3} \phi}
\end{gathered}
$$

e

$$
\Delta_{\phi \phi}^{(2)}=\frac{d_{4}}{2 n^{2} d_{2}^{3}}
$$

Segue portanto que a matriz de covariâncias de segunda ordem dos EMVs corrigidos pelo viés de ordem $n^{-1}$ em MLGs com parâmetro de dispersão desconhecido é dada por

$$
\operatorname{Cov}_{2}(\overline{\boldsymbol{\theta}})=\operatorname{Cov}_{2}(\hat{\boldsymbol{\theta}})+\boldsymbol{\Delta}+\boldsymbol{\Delta}^{\top}
$$

em que $\operatorname{Cov}_{2}(\hat{\boldsymbol{\theta}})=\operatorname{Cov}_{1}(\hat{\boldsymbol{\theta}})+\boldsymbol{\Sigma}$, com a matriz $\operatorname{Cov}_{1}(\hat{\boldsymbol{\theta}})$ definida em (2.7), sendo $\boldsymbol{K}_{\beta}=$ $\phi \boldsymbol{X}^{\top} \boldsymbol{W} \boldsymbol{X}$ e $K_{\lambda}=K_{\phi}=-n d_{2}$, a matriz $\boldsymbol{\Sigma}$ definida em (2.8) e dada pelas expressões (2.24)-(2.32) e a matriz $\boldsymbol{\Delta}$ definida em (2.36) e dada pelas expressões (2.61)-(2.68).

\subsection{Testes de Wald modificados}

Seja $\boldsymbol{\theta}=\left(\theta_{1}, \ldots, \theta_{p+q}\right)^{\top}$ um vetor de parâmetros desconhecidos. Suponha que estamos interessados em testar a hipótese nula $\mathcal{H}_{0}: \boldsymbol{\theta}_{1}=\boldsymbol{\theta}_{1}^{(0)}$ contra a hipótese alternativa $\mathcal{H}_{1}$ : $\boldsymbol{\theta}_{1} \neq \boldsymbol{\theta}_{1}^{(0)}$, em que $\boldsymbol{\theta}=\left(\boldsymbol{\theta}_{1}^{\top}, \boldsymbol{\theta}_{2}^{\top}\right)^{\top}$, sendo $\boldsymbol{\theta}_{1}=\left(\theta_{1}, \ldots, \theta_{p}\right)^{\top}$ um vetor $p$ dimensional e $\boldsymbol{\theta}_{2}=\left(\theta_{p+1}, \ldots, \theta_{p+q}\right)^{\top}$ um vetor $q$ dimensional. Neste caso, $\boldsymbol{\theta}_{2}$ é um vetor de parâmetros de perturbação e $\boldsymbol{\theta}_{1}^{(0)}$ é um vetor de constantes conhecidas. 
A estatística usual do teste de Wald para testarmos a hipótese $\mathcal{H}_{0}$, é expressa na forma

$$
W=\left(\widehat{\boldsymbol{\theta}}_{1}-\boldsymbol{\theta}_{1}^{(0)}\right)^{\top}\left[\widehat{\operatorname{Cov}}_{1}\left(\widehat{\boldsymbol{\theta}}_{1}\right)\right]^{-1}\left(\widehat{\boldsymbol{\theta}}_{1}-\boldsymbol{\theta}_{1}^{(0)}\right),
$$

em que $\widehat{\operatorname{Cov}}_{1}\left(\widehat{\boldsymbol{\theta}}_{1}\right)$ denota a matriz de covariâncias de $\widehat{\boldsymbol{\theta}}_{1}$ avaliada na estimativa de máxima verossimilhança de $\boldsymbol{\theta}_{1}$.

Podemos modificar a estatística (2.69) substituindo o EMV $\widehat{\boldsymbol{\theta}}_{1}$ pelo estimador corrigido $\overline{\boldsymbol{\theta}}_{1}$. Temos então

$$
W_{1}=\left(\overline{\boldsymbol{\theta}}_{1}-\boldsymbol{\theta}_{1}^{(0)}\right)^{\top}\left[\overline{\operatorname{Cov}}_{1}\left(\widehat{\boldsymbol{\theta}}_{1}\right)\right]^{-1}\left(\overline{\boldsymbol{\theta}}_{1}-\boldsymbol{\theta}_{1}^{(0)}\right)
$$

em que $\overline{\operatorname{Cov}_{1}}\left(\widehat{\boldsymbol{\theta}}_{1}\right)$ denota a matriz de covariâncias de $\widehat{\boldsymbol{\theta}}_{1}$ avaliada em $\overline{\boldsymbol{\theta}}_{1}$.

Uma outra modificação na estatística (2.69) resulta em substituir simultaneamente o EMV $\hat{\boldsymbol{\theta}}_{1}$ pelo estimador corrigido $\overline{\boldsymbol{\theta}}_{1}$ e $\widehat{\operatorname{Cov}}_{1}\left(\hat{\boldsymbol{\theta}}_{1}\right)$ pela matriz de covariâncias de segunda ordem dos estimadores corrigidos $\overline{\operatorname{Cov}}_{2}\left(\overline{\boldsymbol{\theta}}_{1}\right)$, avaliada em $\overline{\boldsymbol{\theta}}_{1}$. Obtemos assim,

$$
W_{2}=\left(\overline{\boldsymbol{\theta}}_{1}-\boldsymbol{\theta}_{1}^{(0)}\right)^{\top}\left[\overline{\operatorname{Cov}}_{2}\left(\overline{\boldsymbol{\theta}}_{1}\right)\right]^{-1}\left(\overline{\boldsymbol{\theta}}_{1}-\boldsymbol{\theta}_{1}^{(0)}\right)
$$

A distribuição assintótica de $W, W_{1}$ e $W_{2}$ é $\chi_{p}^{2}$.

\subsection{Resultados numéricos}

Nesta seção, apresentamos alguns resultados de simulações para avaliar o desempenho da matriz de covariâncias de segunda ordem dos EMVs corrigidos pelo viés de ordem $n^{-1}$ e a eficácia das modificações na estatística de Wald. Comparamos as matrizes de covariâncias $\overline{\operatorname{Cov}}_{1}(\widehat{\boldsymbol{\theta}})$ e $\overline{\operatorname{Cov}}_{2}(\overline{\boldsymbol{\theta}})$, com a matriz de covariâncias amostrais do estimador corrigido $\overline{\boldsymbol{\theta}}$. Comparamos também o desempenho de quatro testes, o teste de Wald usual $(W)$, os testes de Wald modificados $\left(W_{1}\right)$ e $\left(W_{2}\right)$ e o teste de Wald bootstrap $\left(W_{\text {boot }}\right)$, o qual será definido abaixo.

O desempenho será avaliado em função da proximidade da probabilidade de rejeição da hipótese nula, sendo esta verdadeira (probabilidade do erro tipo I), aos respectivos níveis nominais dos testes. Avaliamos também o poder dos testes modificados e do teste bootstrap.

As simulações realizadas foram baseadas nos modelos de regressão gama e normal inversa com componentes sistemáticas definidas por

$$
\log \left(\mu_{\ell}\right)=\beta_{1}+\beta_{2} x_{\ell 2} \quad \text { e } \quad \log \left(\phi_{\ell}\right)=\lambda_{1}+\lambda_{2} s_{\ell 2}
$$

em que a covariável $x_{2}$ foi obtida a partir da distribuição $\mathcal{U}(1,2)$ e consideramos $s_{2}=x_{2}$. Para cada tamanho da amostra as covariáveis foram mantidas constantes durante as simulações. Os valores verdadeiros dos parâmetros foram fixados em $\beta_{1}=1 / 2, \beta_{2}=1, \lambda_{1}=2$ e $\lambda_{2}=1$. 
Todas as simulações foram realizadas utilizando a linguagem de programação matricial ox (Doornik, 2006). A maximização do logaritmo da função de verossimilhança com respeito a $\boldsymbol{\beta}$ e $\boldsymbol{\lambda}$ foi feita através do método quasi-Newton BFGS (Nocedal e Wright, 2006) com primeiras derivadas analíticas. A quantidade de réplicas de Monte Carlo e bootstrap foram fixadas em 5000 e 500, respectivamente, e foram considerados os seguintes níveis nominais: $\alpha=10 \%, 5 \%$ e $1 \%$.

Para cada uma das réplicas de Monte Carlo, ajustamos os modelos e calculamos os EMVs $\hat{\boldsymbol{\beta}}$ e $\hat{\boldsymbol{\lambda}}$, os estimadores corrigidos pelo viés $\overline{\boldsymbol{\beta}}$ e $\overline{\boldsymbol{\lambda}}$, os estimadores restritos sob a hipótese nula $\tilde{\boldsymbol{\beta}}$ e $\tilde{\boldsymbol{\lambda}}$, as matrizes $\operatorname{Cov}_{1}(\hat{\boldsymbol{\theta}})$ e $\operatorname{Cov}_{2}(\overline{\boldsymbol{\theta}})$ avaliadas em $\overline{\boldsymbol{\theta}}$ e a matriz de covariâncias amostrais dos EMVs corrigidos $\overline{\boldsymbol{\theta}}^{(1)}, \ldots, \overline{\boldsymbol{\theta}}^{(5000)}$. Para cada tamanho da amostra e cada nível nominal considerado, calculamos as taxas de rejeição de cada teste, isto é, estimamos, via simulação, $P\left(W>z_{\alpha}\right), P\left(W_{1}>z_{\alpha}\right), P\left(W_{2}>z_{\alpha}\right)$ em que $z_{\alpha}$ é o quantil $(1-\alpha)$ da distribuição $\chi_{1}^{2}$ de referência. Em outras palavras, calculamos para cada estatística a porcentagem de vezes em que a hipótese nula foi rejeitada.

Para o teste baseado na estatística $W_{\text {boot }}$, a taxa de rejeição é obtida da probabilidade $P\left(W>\widehat{q}_{(1-\alpha)}\right)$ em que $\widehat{q}_{(1-\alpha)}$ é o quantil bootstrap $(1-\alpha)$ que é estimado por meio do procedimento descrito a seguir. Seja $S$ qualquer estatística usual. Utilizando a técnica bootstrap, é possível encontrar a distribuição empírica dessa estatística a partir da amostra observada $\boldsymbol{t}=\left(t_{1}, \ldots, t_{n}\right)^{\top}$. Essa metodologia consiste em gerar $B$ amostras bootstrap $\left(t_{1}^{* 1}, \ldots, t_{n}^{* B}\right)^{\top}$ a partir da amostra original $\boldsymbol{t}$, considerando como verdadeira a hipótese nula dada na Seção 2.4. Em seguida, calcula-se o valor da estatística $S$ para cada amostra, ou seja, obtém-se $S^{* b}$ para $b=1,2, \ldots, B$. Finalmente, esses valores são colocados em ordem crescente. Fixando o nível de significância $\alpha$, o percentil $1-\alpha$ de $S^{* b}$ é estimado pelo valor $\widehat{q}_{(1-\alpha)}$ tal que $\#\left\{S^{* b} \leq \widehat{q}_{(1-\alpha)}\right\} / B=1-\alpha$. Portanto, o teste rejeita a hipótese nula se $S>\widehat{q}_{(1-\alpha)}$. Maiores informações sobre testes bootstrap podem ser encontradas em Efron e Tibshirani (1993, Capítulo 16).

Para as simulações de poder, as taxas de rejeição foram obtidas sob diferentes hipóteses alternativas. Adicionalmente, na avaliação dos testes $W, W_{1}$ e $W_{2}$, utilizou-se os valores críticos estimados obtidos a partir das simulações de tamanho desses testes em vez dos valores tabelados da distribuição qui-quadrado de referência.

\subsubsection{Modelo gama}

Os resultados de simulação apresentados aqui baseiam-se no modelo de regressão gama com componentes sistemáticas dadas em (2.70).

Na Tabela 2.1 comparamos a $\overline{\operatorname{Cov}}_{1}(\widehat{\boldsymbol{\theta}})$ e a $\overline{\operatorname{Cov}}_{2}(\overline{\boldsymbol{\theta}})$ com a matriz de covariâncias amostrais do EMV corrigido do parâmetro $\boldsymbol{\theta}$. Variamos o tamanho da amostra em $n=20,30,40$ e 50 . A primeira e segunda entradas na Tabela 2.1 são as médias amostrais das expansões assintóticas baseadas nas 5000 réplicas, isto é, $\sum_{j=1}^{5000}\left[\overline{\operatorname{Cov}}_{1}(\widehat{\boldsymbol{\theta}})\right]_{j} / 5000$ e $\sum_{j=1}^{5000}\left[\overline{\operatorname{Cov}}_{2}(\overline{\boldsymbol{\theta}})\right]_{j} / 5000$. A terceira 
entrada dessa tabela são as covariâncias amostrais de $\overline{\boldsymbol{\theta}}^{(1)}, \ldots, \overline{\boldsymbol{\theta}}^{(5000)}$.

Podemos observar na Tabela 2.1 que as quantidades da matriz de covariâncias de segunda ordem dos EMVs corrigidos pelo viés de ordem $n^{-1}, \overline{\operatorname{Cov}}_{2}(\overline{\boldsymbol{\theta}})$, se encontram mais próximas dos valores da matriz de covariâncias amostrais de $\overline{\boldsymbol{\theta}}^{(1)}, \ldots, \overline{\boldsymbol{\theta}}^{(5000)}$ do que as quantidades da matriz de covariâncias de primeira ordem dos EMVs corrigidos, $\overline{\operatorname{Cov}}_{1}(\widehat{\boldsymbol{\theta}})$, para todos os tamanhos da amostra. Percebemos também que em valores absolutos, as variâncias e covariâncias amostrais são maiores que os respectivos valores nas matrizes $\overline{\operatorname{Cov}}_{1}(\widehat{\boldsymbol{\theta}})$ e $\overline{\operatorname{Cov}}_{2}(\overline{\boldsymbol{\theta}})$. Notamos ainda que para $n=30,40$ e 50 os valores da $\overline{\operatorname{Cov}}_{2}(\overline{\boldsymbol{\theta}})$ são praticamente iguais aos da matriz de covariâncias amostrais dos estimadores corrigidos para o parâmetro $\boldsymbol{\beta}$.

Nas Tabelas 2.2, 2.3 e 2.4 apresentamos as taxas de rejeição empíricas e variamos o tamanho da amostra em $n=20,30,40,50,60$ e 70 .

Na Tabela 2.2 consideramos a hipótese nula $\mathcal{H}_{0}: \beta_{2}=1$. Podemos observar que o desempenho do teste $W_{2}$ é bem melhor do que os dos testes $W$ e $W_{1}$, pois apresenta taxas de rejeições mais próximas dos níveis nominais fixados do que as respectivas taxas associadas aos testes $W$ e $W_{1}$. Por exemplo, para $n=50$ e $\alpha=5 \%$, as taxas de rejeição foram $5,76 \%$ para $W_{2}, 6,36 \%$ para $W_{1}$ e $7,04 \%$ para $W$. Observa-se também que os testes $W, W_{1}$ e $W_{2}$ são bastante liberais, principalmente quando o tamanho da amostra é pequeno. Notamos ainda que quando o tamanho da amostra aumenta, as taxas de rejeição empíricas de todos os testes se aproximam do nível nominal, conforme esperado. Percebemos também que até mesmo para $n=70$, o teste de Wald usual apresenta taxas de rejeição acima dos níveis considerados. O teste $W_{\text {boot }}$ apresentou um desempenho melhor do que os demais testes, proporcionando uma redução considerável da distorção de tamanho.

Na Tabela 2.3 a hipótese nula considerada foi $\mathcal{H}_{0}: \lambda_{2}=1$. Podemos notar que os testes $W, W_{1}$ e $W_{2}$ são altamente liberais, pois apresentam taxas de rejeição acima do níveis considerados até mesmo para $n=70$. Observa-se também que o teste $W_{2}$ possui um comportamento melhor que os testes $W$ e $W_{1}$. Novamente, conforme esperado, as taxas de rejeição empírica de todos os testes se aproximam do nível nominal quando o tamanho da amostra aumenta. Mais uma vez, o teste $W_{\text {boot }}$ apresentou um desempenho demasiadamente satisfatório em relação aos demais testes.

Consideramos na Tabela 2.4 a hipótese nula $\mathcal{H}_{0}: \beta_{2}=1, \lambda_{2}=1$. Observamos mais uma vez, conforme esperado, que as taxas de rejeição empírica de todos os testes se aproximam do nível nominal quando o tamanho da amostra aumenta. Nota-se novamente que os testes $W$, $W_{1}$ e $W_{2}$ são extremamente liberais e que o teste $W_{2}$ possui um desempenho melhor que os testes $W$ e $W_{1}$. Podemos perceber também que os testes $W$ e $W_{1}$ encontram-se distantes dos níveis nominais até mesmo para $n=70$. O teste $W_{\text {boot }}$ apresentou uma redução significativa da distorção de tamanho e, consequentemente, um desempenho superior aos demais testes.

Na Tabela 2.5 apresentamos os resultados de simulação para o poder. Fixamos $n=$ 30 e $\alpha=10 \%$ e consideramos a hipótese $\mathcal{H}_{1}: \beta_{2}=\epsilon$, em que diferentes valores de $\epsilon$ foram considerados. Para um dado $\epsilon$ fixado, os poderes dos testes possuem comportamentos 
$\mathrm{n}=\mathbf{2 0}$

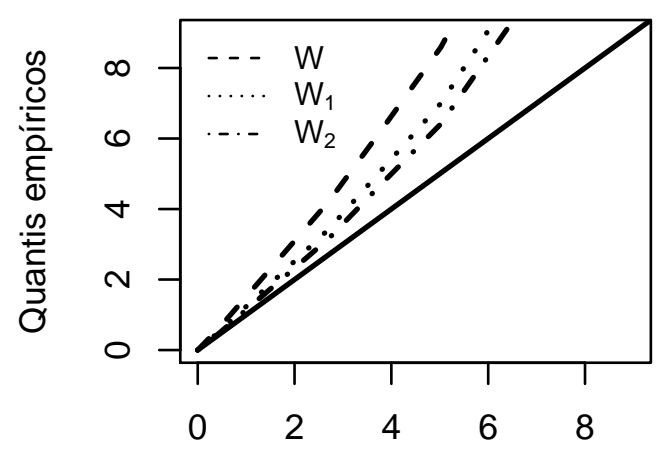

Quantis assintóticos $-\chi_{1}^{2}$

$$
\mathrm{n}=\mathbf{4 0}
$$

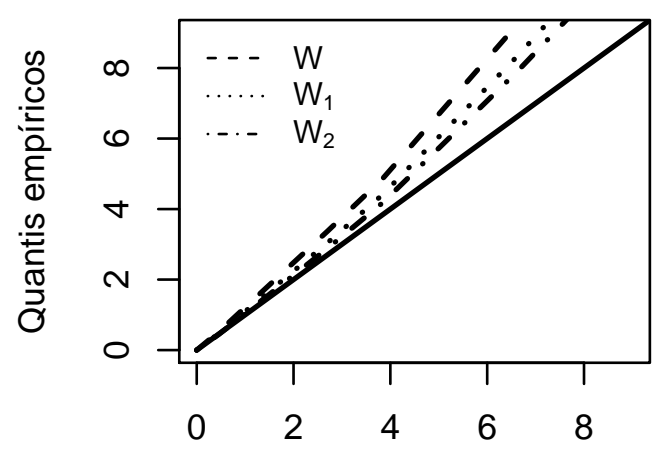

Quantis assintóticos $-\chi_{1}^{2}$ $\mathbf{n}=\mathbf{3 0}$

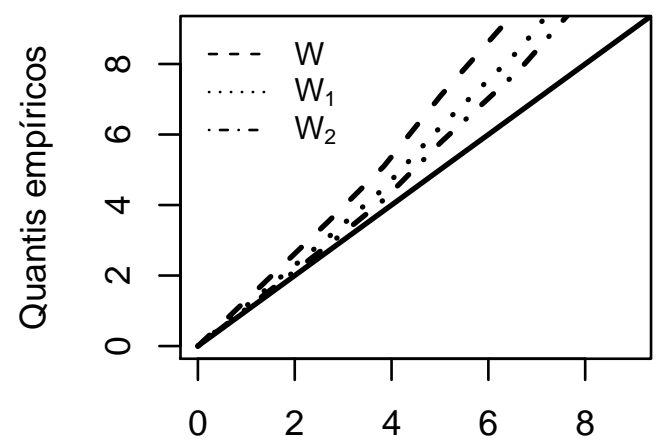

Quantis assintóticos $-\chi_{1}^{2}$

$$
\mathrm{n}=\mathbf{5 0}
$$

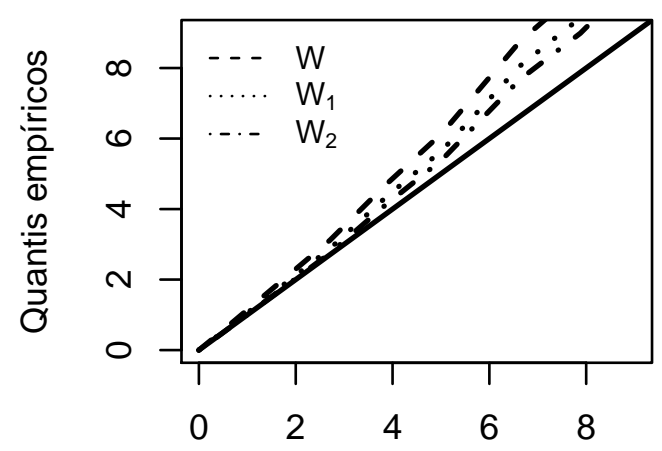

Quantis assintóticos $-\chi_{1}^{2}$

Figura 2.1: Quantis assintóticos versus quantis empíricos das estatísticas $W, W_{1}$ e $W_{2}$ - modelo gama com $p=2, p_{1}=1$ e $q=2$.

semelhantes, sendo $W_{\text {boot }}$ ligeiramente menos poderoso.

Na Figura 2.1 apresentamos gráficos de quantis das estatísticas $W$ (linha tracejada), $W_{1}$ (linha ponto) e $W_{2}$ (linha ponto traço) versus os quantis assintóticos. Consideramos a hipótese nula $\mathcal{H}_{0}: \beta_{2}=1$, ou seja, os quantis assintóticos são obtidos da distribuição qui-quadrado com um grau de liberdade. A identidade é representada pela linha cheia, isto é, os quantis assintóticos são iguais aos quantis empíricos. Observe que a estatística do teste Wald usual apresenta quantis bem maiores que os quantis da distribuição $\chi^{2}$, principalmente quando o tamanho da amostra é pequeno, evidenciando a tendência dessa estatística de rejeitar a hipótese nula com mais frequência que o esperado. Nota-se ainda que as estatísticas de Wald modificadas possuem quantis mais próximos dos quantis da distribuição qui-quadrado em todos os tamanhos da amostra. 
Tabela 2.1: $\overline{\operatorname{Cov}}_{1}(\widehat{\boldsymbol{\theta}}), \overline{\operatorname{Cov}}_{2}(\overline{\boldsymbol{\theta}})$ e covariâncias amostrais para a distribuição gama.

\begin{tabular}{|c|c|c|c|c|c|c|c|c|}
\hline & \multicolumn{4}{|c|}{$n=20$} & \multicolumn{4}{|c|}{$n=30$} \\
\hline & $\beta_{1}$ & $\bar{\beta}_{2}$ & $\overline{\lambda_{1}}$ & $\bar{\lambda}_{2}$ & $\beta_{1}$ & $\bar{\beta}_{2}$ & $\bar{\lambda}_{1}$ & $\bar{\lambda}_{2}$ \\
\hline \multirow{3}{*}{$\beta_{1}$} & 0,0731 & $-0,0479$ & 0 & 0 & 0,0362 & $-0,0222$ & 0 & 0 \\
\hline & 0,0794 & $-0,0521$ & $-0,0124$ & 0,0083 & 0,0389 & $-0,0239$ & $-0,0046$ & 0,0030 \\
\hline & 0,0824 & $-0,0539$ & $-0,0276$ & 0,0202 & 0,0391 & $-0,0240$ & $-0,0061$ & 0,0032 \\
\hline \multirow{3}{*}{$\beta_{2}$} & & 0,0321 & 0 & 0 & & 0,0140 & 0 & 0 \\
\hline & & 0,0349 & 0,0082 & $-0,0056$ & & 0,0150 & 0,0029 & $-0,0019$ \\
\hline & & 0,0360 & 0,0183 & $-0,0134$ & & 0,0151 & 0,0038 & $-0,0019$ \\
\hline \multirow{3}{*}{$\lambda_{1}$} & & & 3,8287 & $-2,5923$ & & & 2,1971 & $-1,4048$ \\
\hline & & & 4,4726 & $-3,0353$ & & & 2,5262 & $-1,6189$ \\
\hline & & & 6,4802 & $-4,4166$ & & & 3,0623 & $-1,9757$ \\
\hline \multirow{5}{*}{$\lambda_{2}$} & & & & 1,8016 & & & & 0,9260 \\
\hline & & & & 2,1064 & & & & 1,0653 \\
\hline & & & & 3,0678 & & & & 1,3076 \\
\hline & & & $=40$ & & & & $=50$ & \\
\hline & $\bar{\beta}_{1}$ & $\bar{\beta}_{2}$ & $\bar{\lambda}_{1}$ & $\bar{\lambda}_{2}$ & $\bar{\beta}_{1}$ & $\bar{\beta}_{2}$ & $\overline{\lambda_{1}}$ & $\bar{\lambda}_{2}$ \\
\hline \multirow{3}{*}{$\beta_{1}$} & 0,0283 & $-0,0172$ & 0 & 0 & 0,0252 & $-0,0153$ & 0 & 0 \\
\hline & 0,0299 & $-0,0182$ & $-0,0027$ & 0,0017 & 0,0264 & $-0,0161$ & $-0,0021$ & 0,0013 \\
\hline & 0,0295 & $-0,0181$ & $-0,0053$ & 0,0036 & 0,0270 & $-0,0164$ & $-0,0028$ & 0,0018 \\
\hline \multirow{3}{*}{$\beta_{2}$} & & 0,0108 & 0 & 0 & & 0,0096 & 0 & 0 \\
\hline & & 0,0114 & 0,0017 & $-0,0011$ & & 0,0100 & 0,0013 & $-0,0008$ \\
\hline & & 0,0113 & 0,0035 & $-0,0024$ & & 0,0103 & 0,0016 & $-0,0010$ \\
\hline \multirow{3}{*}{$\lambda_{1}$} & & & 1,7023 & $-1,0803$ & & & 1,5180 & $-0,9523$ \\
\hline & & & 1,8891 & $-1,2009$ & & & 1,6677 & $-1,0554$ \\
\hline & & & 2,1859 & $-1,3943$ & & & 1,8481 & $-1,1704$ \\
\hline \multirow{3}{*}{$\lambda_{2}$} & & & & 0,7061 & & & & 0,6224 \\
\hline & & & & 0,7840 & & & & 0,6841 \\
\hline & & & & 0,9125 & & & & 0,7588 \\
\hline
\end{tabular}


Tabela 2.2: Taxas de rejeição para $\mathcal{H}_{0}: \beta_{2}=1$; distribuição gama.

\begin{tabular}{|c|c|c|c|c|c|c|c|c|c|c|c|c|}
\hline \multirow[b]{2}{*}{$n$} & \multicolumn{4}{|c|}{$\alpha=10 \%$} & \multicolumn{4}{|c|}{$\alpha=5 \%$} & \multicolumn{4}{|c|}{$\alpha=1 \%$} \\
\hline & $W$ & $W_{1}$ & $W_{2}$ & $W_{\text {boot }}$ & $W$ & $W_{1}$ & $W_{2}$ & $W_{\text {boot }}$ & $W$ & $W_{1}$ & $W_{2}$ & $W_{\text {boot }}$ \\
\hline 20 & 17,94 & 14,00 & 12,62 & 10,68 & 11,62 & 8,40 & 7,32 & 5,56 & 4,52 & 2,84 & 2,28 & 1,42 \\
\hline 30 & 15,38 & 12,78 & 11,34 & 9,88 & 8,90 & 6,94 & 5,94 & 4,88 & 2,92 & 2,18 & 1,80 & 1,04 \\
\hline 40 & 13,58 & 11,82 & 11,00 & 10,22 & 7,92 & 6,48 & 6,06 & 5,34 & 2,68 & 2,04 & 1,74 & 1,36 \\
\hline 50 & 12,08 & 11,00 & 10,18 & 9,72 & 7,04 & 6,36 & 5,76 & 5,14 & 1,96 & 1,66 & 1,46 & 1,20 \\
\hline 60 & 12,96 & 11,84 & 11,04 & 10,46 & 7,30 & 6,62 & 6,16 & 5,90 & 2,06 & 1,70 & 1,36 & 1,20 \\
\hline 70 & 11,56 & 10,42 & 10,08 & 9,90 & 6,10 & 5,48 & 5,10 & 4,80 & 1,46 & 1,24 & 1,14 & 1,04 \\
\hline
\end{tabular}

Tabela 2.3: Taxas de rejeição para $\mathcal{H}_{0}: \lambda_{2}=1$; distribuição gama.

\begin{tabular}{|c|c|c|c|c|c|c|c|c|c|c|c|c|}
\hline \multirow[b]{2}{*}{$n$} & \multicolumn{4}{|c|}{$\alpha=10 \%$} & \multicolumn{4}{|c|}{$\alpha=5 \%$} & \multicolumn{4}{|c|}{$\alpha=1 \%$} \\
\hline & $W$ & $W_{1}$ & $W_{2}$ & $W_{\text {boot }}$ & $W$ & $W_{1}$ & $W_{2}$ & $W_{\text {boot }}$ & $W$ & $W_{1}$ & $W_{2}$ & $W_{\text {boot }}$ \\
\hline 20 & 20,48 & 18,76 & 15,88 & 10,56 & 13,66 & 12,06 & 9,62 & 5,34 & 5,54 & 4,50 & 3,08 & 1,24 \\
\hline 30 & 17,64 & 16,06 & 13,36 & 10,28 & 11,00 & 9,88 & 7,52 & 5,04 & 3,94 & 3,40 & 2,42 & 1,18 \\
\hline 40 & 16,26 & 15,28 & 13,24 & 11,00 & 9,92 & 9,18 & 7,84 & 5,88 & 3,22 & 2,88 & 1,80 & 1,24 \\
\hline 50 & 14,30 & 13,28 & 11,66 & 10,18 & 8,26 & 7,56 & 6,26 & 5,18 & 2,62 & 2,36 & 1,88 & 1,22 \\
\hline 60 & 13,36 & 12,56 & 11,16 & 10,02 & 7,50 & 7,02 & 6,16 & 5,26 & 2,02 & 1,84 & 1,38 & 1,08 \\
\hline 70 & 13,18 & 12,56 & 11,44 & 10,42 & 7,58 & 7,18 & 6,34 & 5,36 & 2,00 & 1,78 & 1,42 & 1,18 \\
\hline
\end{tabular}

Tabela 2.4: Taxas de rejeição para $\mathcal{H}_{0}: \beta_{2}=1, \lambda_{2}=1$; distribuição gama.

\begin{tabular}{|c|c|c|c|c|c|c|c|c|c|c|c|c|}
\hline \multirow[b]{2}{*}{$n$} & \multicolumn{4}{|c|}{$\alpha=10 \%$} & \multicolumn{4}{|c|}{$\alpha=5 \%$} & \multicolumn{4}{|c|}{$\alpha=1 \%$} \\
\hline & $W$ & $W_{1}$ & $W_{2}$ & $W_{\text {boot }}$ & $W$ & $W_{1}$ & $W_{2}$ & $W_{\text {boot }}$ & $W$ & $W_{1}$ & $W_{2}$ & $W_{\text {boot }}$ \\
\hline 20 & 21,96 & 18,50 & 15,44 & 10,14 & 15,40 & 11,98 & 9,40 & 4,98 & 6,78 & 5,18 & 3,88 & 1,22 \\
\hline 30 & 19,88 & 17,12 & 14,52 & 10,76 & 13,20 & 11,00 & 8,72 & 5,26 & 5,22 & 3,92 & 3,08 & 1,20 \\
\hline 40 & 16,56 & 14,56 & 12,50 & 10,18 & 10,02 & 8,36 & 6,92 & 5,06 & 3,38 & 2,74 & 2,10 & 1,20 \\
\hline 50 & 15,58 & 13,96 & 12,54 & 10,56 & 9,70 & 8,30 & 7,06 & 5,62 & 3,10 & 2,50 & 1,96 & 1,16 \\
\hline 60 & 14.38 & 13,20 & 11,96 & 10,52 & 8,42 & 7,54 & 6,80 & 5,66 & 2,70 & 2,12 & 1,70 & 1,14 \\
\hline 70 & 13,44 & 12,24 & 10,76 & 9,88 & 7,32 & 6,76 & 5,90 & 5,02 & 2,36 & 2,02 & 1,56 & 1,10 \\
\hline
\end{tabular}

Tabela 2.5: Poder para $\mathcal{H}_{1}: \beta_{2}=\epsilon ; n=30, \alpha=10 \%$ e distribuição gama.

\begin{tabular}{lcccccccccc}
\hline$\epsilon$ & 0,5 & 0,6 & 0,7 & 0,8 & 0,9 & 1,1 & 1,2 & 1,3 & 1,4 & 1,5 \\
\hline$W$ & 98,76 & 93,92 & 77,14 & 49,20 & 20,88 & 21,06 & 47,18 & 77,22 & 92,50 & 99,00 \\
$W_{1}$ & 98,76 & 93,94 & 77,32 & 49,34 & 20,98 & 21,04 & 47,10 & 77,08 & 92,50 & 99,00 \\
$W_{2}$ & 98,76 & 93,92 & 77,26 & 49,38 & 21,00 & 21,04 & 47,14 & 77,08 & 92,48 & 99,00 \\
$W_{\text {boot }}$ & 98,66 & 93,54 & 76,72 & 48,82 & 20,36 & 20,82 & 46,90 & 76,68 & 92,36 & 98,92 \\
\hline
\end{tabular}




$$
\mathrm{n}=\mathbf{2 0}
$$

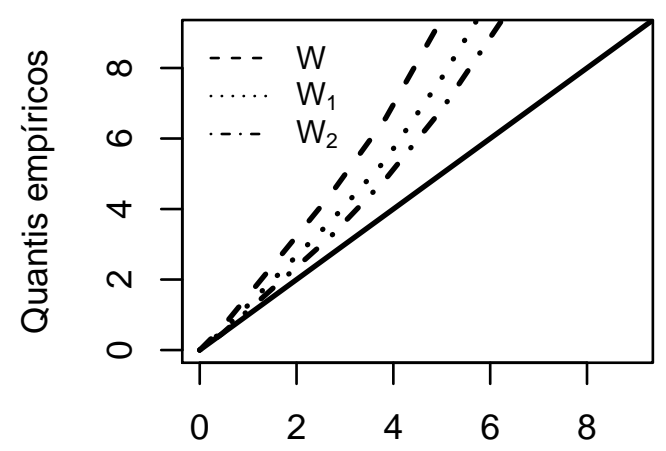

Quantis assintóticos $-\chi_{1}^{2}$

$$
\mathrm{n}=\mathbf{4 0}
$$

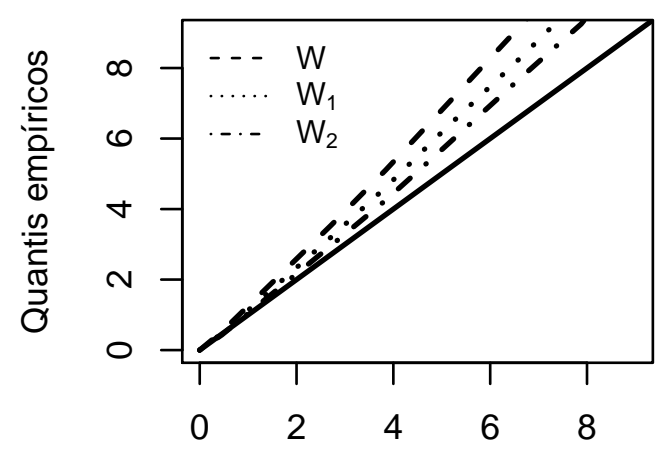

Quantis assintóticos $-\chi_{1}^{2}$ $\mathbf{n}=\mathbf{3 0}$

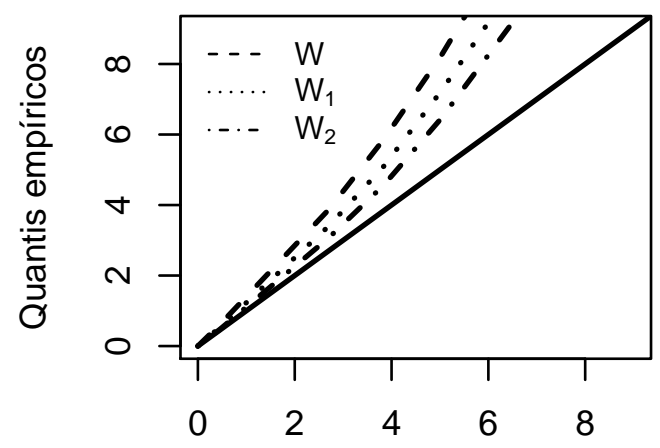

Quantis assintóticos $-\chi_{1}^{2}$

$$
\mathrm{n}=\mathbf{5 0}
$$

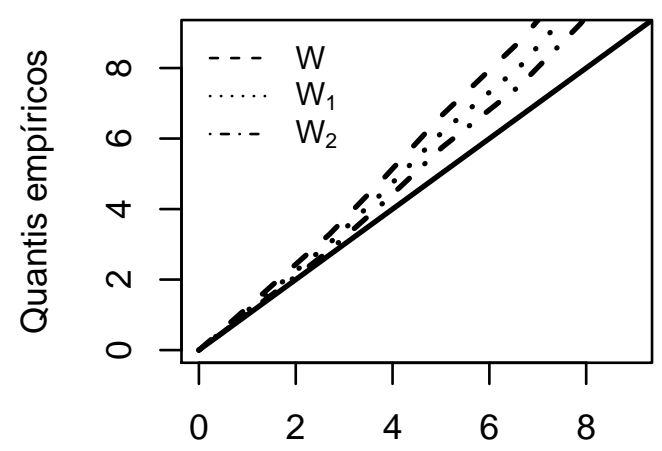

Quantis assintóticos $-\chi_{1}^{2}$

Figura 2.2: Quantis assintóticos versus quantis empíricos das estatísticas $W, W_{1}$ e $W_{2}$ - modelo normal inversa com $p=2, p_{1}=1$ e $q=2$.

\subsubsection{Modelo normal inversa}

Os resultados das simulações apresentados aqui baseiam-se no modelo de regressão normal inversa com componentes sistemáticas dadas em (2.70).

Os comentários feitos na Seção 2.5.1 aplicam-se aqui, exceto os referentes ao poder (Tabela 2.5), uma vez que, nesta seção, as simulações de poder (Tabela 2.10) foram feitas em um cenário diferente.

Na Tabela 2.10 apresentamos os resultados das simulações para o poder. Fixamos $n=30$ e $\alpha=10 \%$ e consideramos a hipótese $\mathcal{H}_{1}: \beta_{2}=\lambda_{2}=\epsilon$, em que diferentes valores de $\epsilon$ foram considerados. Para um dado $\epsilon$ fixado, os poderes dos testes possuem desempenhos similares, sendo $W_{1}$ ligeiramente menos poderoso. 
Tabela 2.6: $\overline{\operatorname{Cov}}_{1}(\widehat{\boldsymbol{\theta}}), \overline{\operatorname{Cov}}_{2}(\overline{\boldsymbol{\theta}})$ e covariâncias amostrais para a distribuição normal inversa.

\begin{tabular}{|c|c|c|c|c|c|c|c|c|}
\hline & \multicolumn{4}{|c|}{$n=20$} & \multicolumn{4}{|c|}{$n=30$} \\
\hline & $\bar{\beta}_{1}$ & $\bar{\beta}_{2}$ & $\bar{\lambda}_{1}$ & $\bar{\lambda}_{2}$ & $\bar{\beta}_{1}$ & $\bar{\beta}_{2}$ & $\bar{\lambda}_{1}$ & $\bar{\lambda}_{2}$ \\
\hline \multirow{3}{*}{$\beta_{1}$} & 0,4621 & $-0,3115$ & 0 & 0 & 0,2558 & $-0,1631$ & 0 & 0 \\
\hline & 0,5263 & $-0,3554$ & $-0,0739$ & 0,0513 & 0,2863 & $-0,1831$ & $-0,0378$ & 0,0249 \\
\hline & 0,5772 & $-0,3917$ & $-0,2938$ & 0,2008 & 0,2976 & $-0,1901$ & $-0,1254$ & 0,0835 \\
\hline \multirow{3}{*}{$\beta_{2}$} & & 0,2158 & 0 & 0 & & 0,1073 & 0 & 0 \\
\hline & & 0,2461 & 0,0510 & $-0,0354$ & & 0,1205 & 0,0249 & $-0,0164$ \\
\hline & & 0,2713 & 0,2008 & $-0,1379$ & & 0,1245 & 0,0806 & $-0,0542$ \\
\hline \multirow{3}{*}{$\lambda_{1}$} & & & 3,8858 & $-2,6298$ & & & 2,2257 & $-1,4224$ \\
\hline & & & 4,5094 & $-3,0627$ & & & 2,5579 & $-1,6412$ \\
\hline & & & 6,2434 & $-4,2523$ & & & 3,1457 & $-2,0274$ \\
\hline \multirow{5}{*}{$\lambda_{2}$} & & & & 1,8267 & & & & 0,9371 \\
\hline & & & & 2,1273 & & & & 1,0812 \\
\hline & & & & 2,9534 & & & & 1,3385 \\
\hline & & & $=40$ & & & & $=50$ & \\
\hline & $\overline{\beta_{1}}$ & $\overline{\beta_{2}}$ & $\overline{\lambda_{1}}$ & $\bar{\lambda}_{2}$ & $\overline{\beta_{1}}$ & $\bar{\beta}_{2}$ & $\overline{\lambda_{1}}$ & $\overline{\lambda_{2}}$ \\
\hline \multirow{3}{*}{$\beta_{1}$} & 0,1972 & $-0,1249$ & 0 & 0 & 0,1744 & $-0,1100$ & 0 & 0 \\
\hline & 0,2143 & $-0,1359$ & $-0,0213$ & 0,0139 & 0,1876 & $-0,1185$ & $-0,0166$ & 0,0108 \\
\hline & 0,2180 & $-0,1390$ & $-0,0560$ & 0,0345 & 0,1873 & $-0,1187$ & $-0,0471$ & 0,0321 \\
\hline \multirow{3}{*}{$\beta_{2}$} & & 0,0815 & 0 & 0 & & 0,0713 & 0 & 0 \\
\hline & & 0,0887 & 0,0139 & $-0,0091$ & & 0,0768 & 0,0108 & $-0,0070$ \\
\hline & & 0,0909 & 0,0366 & $-0,0225$ & & 0,0770 & 0,0305 & $-0,0209$ \\
\hline \multirow{3}{*}{$\lambda_{1}$} & & & 1,7239 & $-1,0935$ & & & 1,5370 & $-0,9709$ \\
\hline & & & 1,9111 & $-1,2158$ & & & 1,6843 & $-1,0664$ \\
\hline & & & 2,1640 & $-1,3852$ & & & 1,8912 & $-1,1984$ \\
\hline \multirow{3}{*}{$\lambda_{2}$} & & & & 0,7144 & & & & 0,6297 \\
\hline & & & & 0,7943 & & & & 0,6916 \\
\hline & & & & 0,9099 & & & & 0,7776 \\
\hline
\end{tabular}


Tabela 2.7: Taxas de rejeição para $\mathcal{H}_{0}: \beta_{1}=1$; distribuição normal inversa.

\begin{tabular}{|c|c|c|c|c|c|c|c|c|c|c|c|c|}
\hline \multirow[b]{2}{*}{$n$} & \multicolumn{4}{|c|}{$\alpha=10 \%$} & \multicolumn{4}{|c|}{$\alpha=5 \%$} & \multicolumn{4}{|c|}{$\alpha=1 \%$} \\
\hline & $W$ & $W_{1}$ & $W_{2}$ & $W_{\text {boot }}$ & $W$ & $W_{1}$ & $W_{2}$ & $W_{\text {boot }}$ & $W$ & $W_{1}$ & $W_{2}$ & $W_{\text {boot }}$ \\
\hline 20 & 18,06 & 14,32 & 12,60 & 10,62 & 12,18 & 9,16 & 7,58 & 5,54 & 4,96 & 3,38 & 2,70 & 1,32 \\
\hline 30 & 15,98 & 13,46 & 11,58 & 10,58 & 10,22 & 8,44 & 7,12 & 5,38 & 3,80 & 2,96 & 2,36 & 1,36 \\
\hline 40 & 14,52 & 12,68 & 11,36 & 10,44 & 8,52 & 7,34 & 6,36 & 5,26 & 2,76 & 2,02 & 1,60 & 1,16 \\
\hline 50 & 13,50 & 11,96 & 10,82 & 10,12 & 7,94 & 6,78 & 5,92 & 5,58 & 2,52 & 2,08 & 1,66 & 1,24 \\
\hline 60 & 13,54 & 12,62 & 11,46 & 11,08 & 7,76 & 6,68 & 5,88 & 5,32 & 1,90 & 1,64 & 1,32 & 1,08 \\
\hline 70 & 13,14 & 11,82 & 10,94 & 10,82 & 7,36 & 6,68 & 6,00 & 5,56 & 2,00 & 1,70 & 1,54 & 1,50 \\
\hline
\end{tabular}

Tabela 2.8: Taxas de rejeição para $\mathcal{H}_{0}: \lambda_{2}=1$; distribuição normal inversa.

\begin{tabular}{|c|c|c|c|c|c|c|c|c|c|c|c|c|}
\hline \multirow[b]{2}{*}{$n$} & \multicolumn{4}{|c|}{$\alpha=10 \%$} & \multicolumn{4}{|c|}{$\alpha=5 \%$} & \multicolumn{4}{|c|}{$\alpha=1 \%$} \\
\hline & $W$ & $W_{1}$ & $W_{2}$ & $W_{\text {boot }}$ & $W$ & $W_{1}$ & $W_{2}$ & $W_{\text {boot }}$ & $W$ & $W_{1}$ & $W_{2}$ & $W_{\text {boot }}$ \\
\hline 20 & 19,84 & 18,02 & 14,66 & 9,92 & 12,90 & 11,50 & 9,26 & 5,14 & 5,56 & 4,54 & 3,58 & 1,16 \\
\hline 30 & 16,96 & 15,52 & 12,94 & 9,54 & 10,34 & 9,34 & 7,36 & 5,14 & 4,32 & 3,56 & 2,52 & 1,32 \\
\hline 40 & 14,68 & 13,56 & 11,70 & 9,60 & 8,56 & 7,94 & 6,36 & 4,92 & 2,94 & 2,56 & 1,96 & 1,30 \\
\hline 50 & 14,70 & 13,64 & 12,08 & 10,34 & 8,70 & 8,10 & 6,78 & 5,52 & 2,82 & 2,46 & 1,90 & 1,34 \\
\hline 60 & 14,32 & 13,52 & 12,14 & 10,86 & 8,34 & 7,80 & 6,70 & 5,82 & 2,42 & 2,24 & 1,82 & 1,34 \\
\hline 70 & 12,70 & 12,00 & 10,78 & 9,76 & 6,80 & 6,38 & 5,76 & 4,90 & 1,98 & 1,80 & 1,50 & 1,28 \\
\hline
\end{tabular}

Tabela 2.9: Taxas de rejeição para $\mathcal{H}_{0}: \beta_{2}=1, \lambda_{2}=1$; distribuição normal inversa.

\begin{tabular}{|c|c|c|c|c|c|c|c|c|c|c|c|c|}
\hline \multirow[b]{2}{*}{$n$} & \multicolumn{4}{|c|}{$\alpha=10 \%$} & \multicolumn{4}{|c|}{$\alpha=5 \%$} & \multicolumn{4}{|c|}{$\alpha=1 \%$} \\
\hline & $W$ & $W_{1}$ & $W_{2}$ & $W_{\text {boot }}$ & $W$ & $W_{1}$ & $W_{2}$ & $W_{\text {boot }}$ & $W$ & $W_{1}$ & $W_{2}$ & $W_{\text {boot }}$ \\
\hline 20 & 22,34 & 18,40 & 15,02 & 9,82 & 15,44 & 12,36 & 9,88 & 4,94 & 7,38 & 5,52 & 4,12 & 1,18 \\
\hline 30 & 20,20 & 17,46 & 14,40 & 10,72 & 13,58 & 11,36 & 9,22 & 5,54 & 5,62 & 4,64 & 3,54 & 1,40 \\
\hline 40 & 16,38 & 14,70 & 12,76 & 10,04 & 10,58 & 8,88 & 7,24 & 5,22 & 3,90 & 3,32 & 2,52 & 1,04 \\
\hline 50 & 15,84 & 14,06 & 12,42 & 10,40 & 9,96 & 8,64 & 7,42 & 5,28 & 3,70 & 2,94 & 2,32 & 1,28 \\
\hline 60 & 15,08 & 13,88 & 12,48 & 10,94 & 9,24 & 8,18 & 6,70 & 5,50 & 2,92 & 2,52 & 2,06 & 1,20 \\
\hline 70 & 13,26 & 11,92 & 10,42 & 9,36 & 7,58 & 6,72 & 5,82 & 4,80 & 2,22 & 1,80 & 1,50 & 1,20 \\
\hline
\end{tabular}

Tabela 2.10: Poder para $\mathcal{H}_{1}: \beta_{2}=\lambda_{2}=\epsilon ; n=30, \alpha=10 \%$ e distribuição normal inversa.

\begin{tabular}{lcccc}
\hline$\epsilon$ & 0,5 & 1,5 & 2,0 & 2,5 \\
\hline$W$ & 31,66 & 32,22 & 80,96 & 99,02 \\
$W_{1}$ & 31,02 & 31,50 & 80,36 & 98,92 \\
$W_{2}$ & 31,76 & 32,06 & 80,94 & 99,06 \\
$W_{\text {boot }}$ & 32,78 & 33,22 & 81,34 & 99,02 \\
\hline
\end{tabular}




\subsection{Aplicação}

Com o intuito de ilustrar o desempenho da estatística de Wald e de suas versões modificadas, consideramos o conjunto de dados analisados em Simonoff e Tsai (1994). Estes dados representam as taxas mensais de retorno de ações de mercado $(x)$ e da Corporação Acme Cleveland (Y), no período de Janeiro de 1986 a Dezembro de 1990. Na análise original destes dados, eles sugeriram um modelo de regressão normal heteroscedástico $N\left(\mu_{\ell}, \sigma_{\ell}^{2}\right)$, sendo $\phi_{\ell}=\sigma_{\ell}^{-2}$, para prever $Y_{\ell}$ em termos de uma função linear do retorno do mercado $x_{\ell}$, considerando as componentes sistemáticas para a média e para a precisão, respectivamente, como $\mu_{\ell}=\beta_{1}+\beta_{2} x_{\ell}$ e $-\log \left(\phi_{\ell}\right)=\lambda_{1}+\lambda_{2} x_{\ell}$, para $\ell=1, \ldots, 59$ em que a observação 22 , a qual reflete que o mercado perdeu aproximadamente um terço de seu valor durante um período de duas semanas no mês de Outubro de 1987 (ver Simonoff e Tsai (1994, p. 359)), foi removida a partir da análise.

Estamos interessados em testar a hipótese nula $\mathcal{H}_{0}: \lambda_{2}=0$ (homocedasticidade).

As estimativas dos parâmetros encontram-se na Tabela 2.11. Podemos observar na Tabela 2.12 que se considerarmos o nível nominal de $5 \%$ a hipótese nula somente é rejeitada quando a inferência é baseada no teste Wald usual. Portanto, as modificações na estatística de Wald e sua versão bootstrap devem ser apreciadas.

Tabela 2.11: Estimativas dos parâmetros e respectivos erros padrões

\begin{tabular}{lcccc}
\hline Parâmetro & $\beta_{1}$ & $\beta_{2}$ & $\lambda_{1}$ & $\lambda_{2}$ \\
\hline Estimativa & $-0,0049$ & 1,2528 & $-4,4104$ & 8,0923 \\
Erro padrão & 0,0197 & 0,2487 & 0,2651 & 4,0088 \\
\hline
\end{tabular}

Tabela 2.12: Valores das estatisticas de teste e valores-p para testar $\mathcal{H}_{0}: \lambda_{2}=0$.

\begin{tabular}{lcccc}
\hline Estatística & $W$ & $W_{1}$ & $W_{2}$ & $W_{\text {boot }}$ \\
\hline valor & 4,0748 & 3,8032 & 3,3916 & \\
valor-p & 0,0435 & 0,0512 & 0,0655 & 0,0775 \\
\hline
\end{tabular}

\subsection{Conclusões}

Nos últimos anos houve um grande interesse em encontrar fórmulas fechadas para os vieses e para as matrizes de covariâncias de segunda ordem dos estimadores de máxima verossimilhança em diversas classes de modelos de regressão. Neste capítulo, consideramos a classe dos MLGHs e obtivemos fórmulas matriciais para a matriz de covariâncias de segunda ordem dos EMVs corrigidos pelo viés de ordem $n^{-1}$. Estas fórmulas juntamente com 
as fórmulas dos EMVs corrigidos foram úteis para a construção de estatísticas de Wald modificadas. Os estudos de simulação indicaram que a matriz de covariâncias de segunda ordem dos EMVs corrigidos pelo viés de ordem $n^{-1}$ possui uma melhor aproximação para o valor verdadeiro da matriz de covariâncias amostrais quando comparada com a matriz de covariâncias de primeira ordem dos EMVs corrigidos. Estes estudos também apontaram que o uso da matriz de covariâncias de segunda ordem juntamente com os EMVs corrigidos produziram uma melhora significativa em relação às distorções de tamanho do teste $W_{2}$ quando comparado com os testes $W$ e $W_{1}$. Os estudos mostraram ainda que o teste $W_{\text {boot }}$ apresentou um desempenho melhor que os demais testes. 


\section{Capítulo 3}

\section{Aperfeiçoamento do teste gradiente}

Neste capítulo, obtivemos os coeficientes do fator de correção tipo-Bartlett para a estatística do teste gradiente em MLGHs. Realizamos estudos de simulação com o objetivo de analisar o desempenho do teste gradiente corrigido para testar hipóteses compostas em pequenas amostras. Comparamos este teste com os seguintes testes: testes da razão de verossimilhanças usual, ajustada e corrigida, testes escore usual e corrigido, testes Wald usual e modificado, teste gradiente usual, testes da razão de verossimilhanças, escore, Wald e gradiente bootstrap. Por fim, aplicamos o resultado obtido a um conjunto de dados reais.

\subsection{Introdução}

Os testes frequentemente utilizados para grandes amostras baseiam-se nas estatísticas da razão de verossimilhanças (Wilks, 1938), Wald (Wald, 1943) e escore (Rao, 1947). Uma estatística alternativa para estes testes em grandes amostras foi proposta por Terrell (2002) e denominada de estatística gradiente. Esta estatística foi derivada a partir das estatísticas escore e de Wald mas, ao contrário das duas, ela não depende da matriz de informação de Fisher, tornando o seu cálculo bastante simples. A estatística gradiente foi recentemente citada por Rao (2005) : "The sugestion by Terrel is attractive as it is simple to compute. It would be of interest to investigate the performance of the [gradient] statistic". Assim como as demais estatísticas (razão de verossimilhanças, Wald e escore), a estatística gradiente possui distribuição assintótica qui-quadrado $(\chi)$ sob a hipótese nula, mas ao contrário das estatísticas da razão de verossimilhanças e escore, ela não é invariante sob reparametrização do modelo que preserva o parâmetro de interesse, assim como a Wald.

Por se tratar de uma estatística recente, a estatística gradiente vem sendo tema de pesquisas atualmente. Lemonte e Ferrari (2012a) obtiveram o poder local do teste gradiente sob alternativas de Pitman (uma sequência de hipóteses alternativas convergindo para a hipótese nula com taxa $n^{-1 / 2}$ ). Eles compararam o poder local do teste gradiente com o dos testes da razão de verossimilhanças, Wald e escore e demonstraram que nenhum dos testes é uniformemente mais poderoso que os outros. Podemos citar também o estudo do poder 
local dos quatro testes na classe dos modelos não lineares da familia exponencial (Lemonte , 2011), modelos de regressão Birnbaum-Saunders (Lemonte e Ferrari, 2011), modelos de regressão lineares simétricos (Lemonte, 2012), modelos de dispersão (Lemonte e Ferrari , 2012b), modelos lineares generalizados com dispersão variável (Lemonte, 2013), modelos de regressão exponencial censurados (Lemonte, 2014a) e modelos não lineares de efeitos mistos (Lemonte, 2014b). Maiores informações sobre a estatística gradiente podem ser encontradas em Lemonte (2016).

Conforme mencionado anteriormente, a estatística gradiente, assim como as outras (razão de verossimilhanças, Wald e escore), possui distribuição assintótica $\chi^{2}$ sob a hipótese nula. Entretanto, sabemos que em pequenas amostras a aproximação $\chi^{2}$ usual para a distribuição destas estatísticas pode não ser satisfatória. Com o intuito de melhorar esta aproximação, houve na literatura a necessidade de sugerir métodos de aperfeiçoamento dos testes baseandose em estatísticas modificadas, como por exemplo, fatores de correção de Bartlett e tipoBartlett e ajuste de Skovgaard.

O fator de correção tipo-Bartlett para a estatística gradiente foi obtido por Vargas et al. (2013). A partir de seus resultados, eles derivaram expressões de forma fechada para o fator de correção tipo-Bartlett que define a estatística gradiente corrigida na classe dos MLGs (Vargas et al., 2014).

O objetivo deste capítulo é obter a correção tipo-Bartlett para a estatística gradiente em MLGHs, generalizando assim, os resultados de Vargas et al. (2014).

\subsection{Teste gradiente aperfeiçoado}

Seja $\boldsymbol{\theta}=\left(\theta_{1}, \ldots, \theta_{r}\right)^{\top}$ um vetor de parâmetros desconhecidos. Vamos considerar o problema de testar a hipótese nula do tipo $\mathcal{H}_{0}: \boldsymbol{\theta}_{1}=\boldsymbol{\theta}_{1}^{(0)}$ contra a hipótese alternativa $\mathcal{H}_{1}: \boldsymbol{\theta}_{1} \neq \boldsymbol{\theta}_{1}^{(0)}$, em que $\boldsymbol{\theta}=\left(\boldsymbol{\theta}_{1}^{\top}, \boldsymbol{\theta}_{2}^{\top}\right)^{\top}$, sendo $\boldsymbol{\theta}_{1}=\left(\theta_{1}, \ldots, \theta_{s}\right)^{\top}$ um vetor $s$ dimensional e $\boldsymbol{\theta}_{2}=\left(\theta_{s+1}, \ldots, \theta_{r}\right)^{\top}$ um vetor $r-s$ dimensional. Neste caso, $\boldsymbol{\theta}_{2}$ é um vetor de parâmetros de perturbação e $\boldsymbol{\theta}_{1}^{(0)}$ é um vetor de constantes conhecidas. A partição de $\boldsymbol{\theta}$ induz a seguinte partição: $\boldsymbol{U}(\boldsymbol{\theta})=\left(\boldsymbol{U}_{1}(\boldsymbol{\theta})^{\top}, \boldsymbol{U}_{2}(\boldsymbol{\theta})^{\top}\right)^{\top}$. Considere $\widehat{\boldsymbol{\theta}}=\left(\widehat{\boldsymbol{\theta}}_{1}^{\top}, \widehat{\boldsymbol{\theta}}_{2}^{\top}\right)^{\top}$ o estimador de máxima verossimilhança irrestrito de $\boldsymbol{\theta}$ e $\widetilde{\boldsymbol{\theta}}=\left(\boldsymbol{\theta}_{1}^{(0) \top}, \tilde{\boldsymbol{\theta}}_{2}^{\top}\right)^{\top}$ o estimador de máxima verossimilhança restrito de $\boldsymbol{\theta}$ sob a hipótese nula.

A estatística gradiente para testar $\mathcal{H}_{0}$ contra $\mathcal{H}_{1}$ é definida como

$$
T=\boldsymbol{U}(\widetilde{\boldsymbol{\theta}})^{\top}(\widehat{\boldsymbol{\theta}}-\widetilde{\boldsymbol{\theta}})
$$

e pode ser expressa como $T=\boldsymbol{U}_{1}(\widetilde{\boldsymbol{\theta}})^{\top}\left(\widehat{\boldsymbol{\theta}}_{1}-\boldsymbol{\theta}_{1}^{(0)}\right)$, uma vez que $\boldsymbol{U}_{2}(\widetilde{\boldsymbol{\theta}})=\mathbf{0}$. Em grandes amostras, a estatística $T$ possui, sob a hipótese nula, distribuição $\chi_{s}^{2}$, em que $s$ é o número de restrições impostas em $\mathcal{H}_{0}$. 
A estatística gradiente modificada é definida como

$$
T_{\mathrm{b}}=T\left[1-\left(c+b T+a T^{2}\right)\right]
$$

em que

$$
a=\frac{A_{3}}{12 s(s+2)(s+4)}, \quad b=\frac{A_{2}-2 A_{3}}{12 s(s+2)}, \quad c=\frac{A_{1}-A_{2}+A_{3}}{12 s}
$$

com as quantidades $A_{1}, A_{2}$ e $A_{3}$ dadas em Vargas et al. (2013) por

$$
\begin{aligned}
A_{1}= & 3 \sum \kappa_{r s t} \kappa_{u v w}\left[m^{r s} a^{v w}\left(m^{t u}+2 a^{t u}\right)+a^{r s} m^{t u} a^{v w}+2 m^{r u} a^{s v} a^{t w}\right] \\
& -12 \sum \kappa_{r s}^{(t)} \kappa_{u v}^{(w)}\left(\kappa^{r t} \kappa^{s u} \kappa^{v w}+a^{r t} a^{s u} a^{v w}+\kappa^{t u} \kappa^{r v} \kappa^{s w}+a^{t u} a^{r v} a^{s w}\right) \\
& -6 \sum \kappa_{r s t} \kappa_{u v}^{(w)}\left[\left(a^{t w}-\kappa^{t w}\right)\left(\kappa^{r u} \kappa^{s v}-a^{r u} a^{s v}\right)+m^{r s}\left(a^{t u} a^{v w}+\kappa^{t u} \kappa^{v w}\right)\right. \\
& \left.+2 a^{s t}\left(\kappa^{r u} \kappa^{v w}-a^{r u} a^{v w}\right)+2 a^{s u} a^{t v} m^{r w}\right] \\
& +6 \sum \kappa_{r s t u} m^{r s} a^{t u}-6 \sum \kappa_{r s t}^{(u)}\left[m^{r s}\left(a^{t u}-\kappa^{t u}\right)+2 m^{r u} a^{s t}\right] \\
& +12 \sum \kappa_{s t}^{(r u)}\left(\kappa^{r s} \kappa^{t u}-a^{r s} a^{t u}\right), \\
A_{2}=- & 3 \sum \kappa_{r s t} \kappa_{u v w}\left[\frac{1}{4} m^{r s} m^{t u}\left(4 a^{v w}+3 m^{w v}\right)+m^{r s} a^{t u} m^{v w}+\frac{1}{2} m^{r u} m^{s v}\left(4 a^{t w}+m^{t w}\right)\right] \\
+ & 6 \sum \kappa_{r s t} \kappa_{u v}^{(w)}\left[m^{t w}\left(\kappa^{r u} \kappa^{s v}-a^{r u} a^{s v}\right)+m^{r s}\left(\kappa^{t u} \kappa^{v w}-a^{t u} a^{v w}\right)\right] \\
+ & 6 \sum \kappa_{r s t}^{(u)} m^{r s} m^{t u}-3 \sum \kappa_{r s t u} m^{r s} m^{t u},
\end{aligned}
$$

em que o somatório se estende sobre todos os $r$ parâmetros $\theta_{1}, \ldots, \theta_{r}$ e $-\kappa_{i j},-\kappa^{i j}, a^{i j}$ e $m^{i j}$ são, respectivamente, os elementos $(i, j)$ das matrizes

$\boldsymbol{K}(\boldsymbol{\theta})=\left[\begin{array}{ll}\boldsymbol{K}_{11} & \boldsymbol{K}_{12} \\ \boldsymbol{K}_{21} & \boldsymbol{K}_{22}\end{array}\right], \quad \boldsymbol{K}^{-1}(\boldsymbol{\theta})=\left[\begin{array}{ll}\boldsymbol{K}^{11} & \boldsymbol{K}^{12} \\ \boldsymbol{K}^{21} & \boldsymbol{K}^{22}\end{array}\right], \quad \boldsymbol{A}=\left[\begin{array}{cc}\mathbf{0} & \mathbf{0} \\ \mathbf{0} & \boldsymbol{K}_{22}^{-1}\end{array}\right] \quad$ e $\quad \boldsymbol{M}=\boldsymbol{K}^{-1}(\boldsymbol{\theta})-\boldsymbol{A}$.

Quando os coeficientes $a, b$ e $c$ envolvem parâmetros desconhecidos, eles devem ser substituídos por suas estimativas de máxima verossimilhança sob a hipótese nula. 


\subsection{Teste gradiente aperfeiçoado em modelos lineares ge- neralizados heteroscedásticos}

Nesta seção apresentamos fatores de correções tipo-Bartlett para a estatística gradiente em testes de hipóteses sobre o vetor de parâmetros $\boldsymbol{\theta}=\left(\boldsymbol{\beta}^{\top}, \boldsymbol{\lambda}^{\top}\right)^{\top}$ em MLGHs, que são de grande interesse na prática. Primeiramente, derivamos um fator de correção tipo-Bartlett para testes de hipóteses sobre dois subconjuntos dos vetores de parâmetros $\boldsymbol{\beta}$ e $\boldsymbol{\lambda}$. Em seguida, derivamos um fator de correção tipo-Bartlett para testar um subconjunto dos parâmetros $\boldsymbol{\beta}$ e, por fim, apresentamos um fator de correção para o teste sobre um subconjunto do vetor de parâmetros $\boldsymbol{\lambda}$.

\subsubsection{Hipótese sobre subconjuntos de $\beta$ e $\lambda$}

Suponha que estamos interessados em testar ambos os efeitos na média e na dispersão, isto é, consideramos a hipótese nula $\mathcal{H}_{0}: \boldsymbol{\beta}_{1}=\boldsymbol{\beta}_{1}^{(0)}, \boldsymbol{\lambda}_{1}=\boldsymbol{\lambda}_{1}^{(0)}$ que será testada contra a hipótese alternativa $\mathcal{H}_{1}$ : violação de pelo menos uma das igualdades, em que os vetores $\boldsymbol{\beta}$ e $\boldsymbol{\lambda}$ são particionados das seguintes maneiras: $\boldsymbol{\beta}=\left(\boldsymbol{\beta}_{1}^{\top}, \boldsymbol{\beta}_{2}^{\top}\right)^{\top}, \operatorname{com} \boldsymbol{\beta}_{1}=\left(\beta_{1}, \ldots, \beta_{p_{1}}\right)^{\top}$ e $\boldsymbol{\beta}_{2}=\left(\beta_{p_{1}+1}, \ldots, \beta_{p}\right)^{\top}, \boldsymbol{\lambda}=\left(\boldsymbol{\lambda}_{1}^{\top}, \boldsymbol{\lambda}_{2}^{\top}\right)^{\top}, \operatorname{com} \boldsymbol{\lambda}_{1}=\left(\lambda_{1}, \ldots, \lambda_{q_{1}}\right)^{\top}$ e $\boldsymbol{\lambda}_{2}=\left(\lambda_{q_{1}+1}, \ldots, \lambda_{q}\right)^{\top}$. Os vetores $\boldsymbol{\beta}_{1}^{(0)}$ e $\boldsymbol{\lambda}_{1}^{(0)}$ são especificados e de dimensões $p_{1}$ e $q_{1}$, respectivamente. Já os vetores $\boldsymbol{\beta}_{2}$ e $\boldsymbol{\lambda}_{2}$ atuam como parâmetros de perturbação.

Devido à ortogonalidade global entre $\boldsymbol{\beta}$ e $\boldsymbol{\lambda}$, a matriz de informação total de Fisher $\boldsymbol{K}(\boldsymbol{\theta})$ para $\boldsymbol{\theta}$ e sua inversa $\boldsymbol{K}^{-1}(\boldsymbol{\theta})$ são bloco-diagonais e podem ser escritas como

$$
\boldsymbol{K}(\boldsymbol{\theta})=\left[\begin{array}{cc}
\boldsymbol{K}_{\beta} & \mathbf{0} \\
\mathbf{0} & \boldsymbol{K}_{\lambda}
\end{array}\right] \quad \text { e } \quad \boldsymbol{K}^{-1}(\boldsymbol{\theta})=\left[\begin{array}{cc}
\boldsymbol{K}_{\beta}^{-1} & \mathbf{0} \\
\mathbf{0} & \boldsymbol{K}_{\lambda}^{-1}
\end{array}\right]
$$

A partição de $\boldsymbol{\beta}=\left(\boldsymbol{\beta}_{1}^{\top}, \boldsymbol{\beta}_{2}^{\top}\right)^{\top}$ e $\boldsymbol{\lambda}=\left(\boldsymbol{\lambda}_{1}^{\top}, \boldsymbol{\lambda}_{2}^{\top}\right)^{\top}$ induz às seguintes partições

$$
\begin{aligned}
& \boldsymbol{K}_{\beta}=\left[\begin{array}{ll}
\boldsymbol{K}_{\beta 11} & \boldsymbol{K}_{\beta 12} \\
\boldsymbol{K}_{\beta 21} & \boldsymbol{K}_{\beta 22}
\end{array}\right], \quad \boldsymbol{K}_{\beta}^{-1}=\left[\begin{array}{cc}
\boldsymbol{K}_{\beta}^{11} & \boldsymbol{K}_{\beta}^{12} \\
\boldsymbol{K}_{\beta}^{21} & \boldsymbol{K}_{\beta}^{22}
\end{array}\right] \\
& \boldsymbol{K}_{\lambda}=\left[\begin{array}{ll}
\boldsymbol{K}_{\lambda 11} & \boldsymbol{K}_{\lambda 12} \\
\boldsymbol{K}_{\lambda 21} & \boldsymbol{K}_{\lambda 22}
\end{array}\right] \quad \text { e } \quad \boldsymbol{K}_{\lambda}^{-1}=\left[\begin{array}{cc}
\boldsymbol{K}_{\lambda}^{11} & \boldsymbol{K}_{\lambda}^{12} \\
\boldsymbol{K}_{\lambda}^{21} & \boldsymbol{K}_{\lambda}^{22}
\end{array}\right]
\end{aligned}
$$

em que $\boldsymbol{K}_{\beta}$ e $\boldsymbol{K}_{\lambda}$ são as matrizes de informação de Fisher de $\boldsymbol{\beta}$ e $\boldsymbol{\lambda}$, respectivamente; $\boldsymbol{K}_{\beta}^{-1}$ e $\boldsymbol{K}_{\lambda}^{-1}$ são suas inversas. 
Consideramos as matrizes $\boldsymbol{A}$ e $\boldsymbol{M}$ particionadas das seguintes maneiras:

$$
\begin{aligned}
& \boldsymbol{A}=\left[\begin{array}{cc}
\boldsymbol{A}_{\beta} & \mathbf{0} \\
\mathbf{0} & \boldsymbol{A}_{\lambda}
\end{array}\right] \quad \text { e } \boldsymbol{M}=\left[\begin{array}{cc}
\boldsymbol{M}_{\beta} & \mathbf{0} \\
\mathbf{0} & \boldsymbol{M}_{\lambda}
\end{array}\right] \\
& \boldsymbol{A}_{\beta}=\left[\begin{array}{cc}
\mathbf{0} & \mathbf{0} \\
\mathbf{0} & \boldsymbol{K}_{\beta 22}^{-1}
\end{array}\right], \quad \boldsymbol{A}_{\lambda}=\left[\begin{array}{cc}
\mathbf{0} & \mathbf{0} \\
\mathbf{0} & \boldsymbol{K}_{\lambda 22}^{-1}
\end{array}\right], \\
& \boldsymbol{M}_{\beta}=\boldsymbol{K}_{\beta}^{-1}-\boldsymbol{A}_{\beta} \quad \text { e } \quad \boldsymbol{M}_{\lambda}=\boldsymbol{K}_{\lambda}^{-1}-\boldsymbol{A}_{\lambda} .
\end{aligned}
$$

Definimos as seguintes matrizes:

$$
\begin{gathered}
\boldsymbol{Z}_{\beta}=\left\{z_{\beta \ell m}\right\}=\boldsymbol{X}\left(\boldsymbol{X}^{\top} \boldsymbol{W} \boldsymbol{\Phi} \boldsymbol{X}\right)^{-1} \boldsymbol{X}^{\top}, \quad \boldsymbol{Z}_{\lambda}=\left\{z_{\lambda \ell m}\right\}=\boldsymbol{S}\left(-\boldsymbol{S}^{\top} \boldsymbol{D}_{2} \boldsymbol{\Phi}_{1}^{2} \boldsymbol{S}\right)^{-1} \boldsymbol{S}^{\top}, \\
\boldsymbol{Z}_{\beta d}=\operatorname{diag}\left(z_{\beta 11}, \ldots, z_{\beta n n}\right), \quad \boldsymbol{Z}_{\lambda d}=\operatorname{diag}\left(z_{\lambda 11}, \ldots, z_{\lambda n n}\right), \\
\boldsymbol{Z}_{\beta_{2}}=\left\{z_{\beta_{2} \ell m}\right\}=\boldsymbol{X}_{2}\left(\boldsymbol{X}_{2}^{\top} \boldsymbol{W} \boldsymbol{\Phi} \boldsymbol{X}_{\mathbf{2}}\right)^{-1} \boldsymbol{X}_{2}^{\top}, \quad \boldsymbol{Z}_{\lambda_{2}}=\left\{z_{\lambda_{2} \ell m}\right\}=\boldsymbol{S}_{2}\left(-\boldsymbol{S}_{2}^{\top} \boldsymbol{D}_{2} \boldsymbol{\Phi}_{1}^{2} \boldsymbol{S}_{2}\right)^{-1} \boldsymbol{S}_{2}^{\top}, \\
\boldsymbol{Z}_{\beta_{2} d}=\operatorname{diag}\left(z_{\beta_{2} 11}, \ldots, z_{\beta_{2} n n}\right), \quad \boldsymbol{Z}_{\lambda_{2} d}=\operatorname{diag}\left(z_{\lambda_{2} 11}, \ldots, z_{\lambda_{2} n n}\right), \\
\boldsymbol{F}=\operatorname{diag}\left\{f_{1}, \ldots, f_{n}\right\}, \quad \boldsymbol{G}_{=1} \operatorname{diag}\left\{g_{1}, \ldots, g_{n}\right\}, \\
\boldsymbol{B}=\operatorname{diag}\left\{b_{1}, \ldots, b_{n}\right\}, \quad \boldsymbol{C}=\operatorname{diag}\left\{c_{1}, \ldots, c_{n}\right\}, \quad \boldsymbol{E}=\operatorname{diag}\left\{e_{1}, \ldots, e_{n}\right\}, \\
\boldsymbol{\Phi}_{1}=\operatorname{diag}\left\{\phi_{11}, \ldots, \phi_{1 n}\right\}, \quad \boldsymbol{\Phi}_{2}=\operatorname{diag}\left\{\phi_{21}, \ldots, \phi_{2 n}\right\}, \quad \boldsymbol{\Phi}_{3}=\operatorname{diag}\left\{\phi_{31}, \ldots, \phi_{3 n}\right\}, \\
\operatorname{diag}\left\{d_{21}, \ldots, d_{2 n}\right\}, \quad \boldsymbol{D}_{3}=\operatorname{diag}\left\{d_{31}, \ldots, d_{3 n}\right\} \quad \mathrm{e} \quad \boldsymbol{D}_{4}=\operatorname{diag}\left\{d_{41}, \ldots, d_{4 n}\right\},
\end{gathered}
$$

com

$$
f_{\ell}=\frac{1}{V_{\ell}} \frac{\mathrm{d} \mu_{\ell}}{\mathrm{d} \eta_{\ell}} \frac{\mathrm{d}^{2} \mu_{\ell}}{\mathrm{d} \eta_{\ell}^{2}}, \quad g_{\ell}=f_{\ell}-\frac{1}{V_{\ell}^{2}} \frac{\mathrm{d} V_{\ell}}{\mathrm{d} \mu_{\ell}}\left(\frac{\mathrm{d} \mu_{\ell}}{\mathrm{d} \eta_{\ell}}\right)^{3},
$$




$$
\begin{gathered}
\nu_{1 \ell}=\frac{1}{V_{\ell}^{3}}\left(\frac{\mathrm{d} V_{\ell}}{\mathrm{d} \mu_{\ell}}\right)^{2}\left(\frac{\mathrm{d} \mu_{\ell}}{\mathrm{d} \eta_{\ell}}\right)^{4}, \quad \nu_{2 \ell}=\frac{1}{V_{\ell}^{2}} \frac{\mathrm{d}^{2} V_{\ell}}{\mathrm{d} \mu_{\ell}^{2}}\left(\frac{\mathrm{d} \mu_{\ell}}{\mathrm{d} \eta_{\ell}}\right)^{4}, \quad \nu_{3 \ell}=\frac{1}{V_{\ell}^{2}} \frac{\mathrm{d} V_{\ell}}{\mathrm{d} \mu_{\ell}}\left(\frac{\mathrm{d} \mu_{\ell}}{\mathrm{d} \eta_{\ell}}\right)^{2} \frac{\mathrm{d}^{2} \mu_{\ell}}{\mathrm{d} \eta_{\ell}{ }^{2}}, \\
\nu_{4 \ell}=\frac{1}{V_{\ell}}\left(\frac{\mathrm{d}^{2} \mu_{\ell}}{\mathrm{d} \eta_{\ell}^{2}}\right)^{2}, \quad \nu_{5 \ell}=\frac{1}{V_{\ell}} \frac{\mathrm{d} \mu_{\ell}}{\mathrm{d} \eta_{\ell}} \frac{\mathrm{d}^{3} \mu_{\ell}}{\mathrm{d} \eta_{\ell}^{3}} \\
b_{\ell}=-6 \nu_{1 \ell}+3 \nu_{2 \ell}+12 \nu_{3 \ell}-3 \nu_{4 \ell}-4 \nu_{5 \ell}, \quad c_{\ell}=-4 \nu_{1 \ell}+2 \nu_{2 \ell}+9 \nu_{3 \ell}-3 \nu_{4 \ell}-3 \nu_{5 \ell}, \\
e_{\ell}=-2 \nu_{1 \ell}+\nu_{2 \ell}+5 \nu_{3 \ell}-2 \nu_{4 \ell}-2 \nu_{5 \ell}
\end{gathered}
$$

e

$$
\phi_{k \ell}=\mathrm{d}^{k} \phi_{\ell} / \mathrm{d} \tau_{\ell}^{k} \text { e } d_{k \ell}=\mathrm{d}^{k} d\left(\phi_{\ell}\right) / \mathrm{d} \phi_{\ell}^{k}, \text { para } k=1, \ldots, 4 \text { e } \ell=1, \ldots, n
$$

Considere $\boldsymbol{Z}_{\beta}^{(2)}=\boldsymbol{Z}_{\beta} \odot \boldsymbol{Z}_{\beta}, \boldsymbol{Z}_{\beta}^{(3)}=\boldsymbol{Z}_{\beta}^{(2)} \odot \boldsymbol{Z}_{\beta}$, etc, em que o símbolo $\odot$ denota o produto de Hadamard (produto direto) entre matrizes.

Desenvolvendo as expressões (3.4)-(3.6) e, após alguma álgebra, obtemos

$$
A_{1}=A_{11}+A_{12}+A_{13}+A_{14}
$$

sendo

$$
\begin{aligned}
& A_{11}=3\left\{\mathbf{1}^{\top} \boldsymbol{\Phi}(\boldsymbol{F}+2 \boldsymbol{G})\left[\boldsymbol{Z}_{\beta d}\left(\boldsymbol{Z}_{\beta}+\boldsymbol{Z}_{\beta_{2}}\right) \boldsymbol{Z}_{\beta_{2} d}-2 \boldsymbol{Z}_{\beta_{2} d} \boldsymbol{Z}_{\beta_{2}} \boldsymbol{Z}_{\beta_{2} d}+2\left(\boldsymbol{Z}_{\beta}-\boldsymbol{Z}_{\beta_{2}}\right) \odot \boldsymbol{Z}_{\beta_{2}}^{(2)}\right]\right. \\
& \times \boldsymbol{\Phi}(\boldsymbol{F}+2 \boldsymbol{G}) \mathbf{1}\} \\
& +3\left\{\mathbf { 1 } ^ { \top } \boldsymbol { \Phi } _ { 1 } \boldsymbol { W } \left[\boldsymbol{Z}_{\beta d}\left(\boldsymbol{Z}_{\lambda}+\boldsymbol{Z}_{\lambda_{2}}\right) \boldsymbol{Z}_{\beta_{2} d}-2 \boldsymbol{Z}_{\beta_{2} d} \boldsymbol{Z}_{\lambda_{2}} \boldsymbol{Z}_{\beta_{2} d}+4\left(\boldsymbol{Z}_{\beta}-\boldsymbol{Z}_{\beta_{2}}\right) \odot \boldsymbol{Z}_{\beta_{2}} \odot \boldsymbol{Z}_{\lambda_{2}}\right.\right. \\
& \left.\left.+2\left(\boldsymbol{Z}_{\lambda}-\boldsymbol{Z}_{\lambda_{2}}\right) \odot \boldsymbol{Z}_{\beta_{2}}^{(2)}\right] \boldsymbol{\Phi}_{1} \boldsymbol{W} \mathbf{1}\right\} \\
& -3\left\{\mathbf{1}^{\top} \boldsymbol{\Phi}_{1} \boldsymbol{W}\left[\boldsymbol{Z}_{\beta d}\left(\boldsymbol{Z}_{\lambda}+\boldsymbol{Z}_{\lambda_{2}}\right) \boldsymbol{Z}_{\lambda_{2} d}-2 \boldsymbol{Z}_{\beta_{2} d} \boldsymbol{Z}_{\lambda_{2}} \boldsymbol{Z}_{\lambda_{2} d}\right]\left(\boldsymbol{D}_{3} \boldsymbol{\Phi}_{1}^{3}+3 \boldsymbol{D}_{2} \boldsymbol{\Phi}_{1} \boldsymbol{\Phi}_{2}\right) \mathbf{1}\right\} \\
& -3\left\{\mathbf{1}^{\top}\left(\boldsymbol{D}_{3} \boldsymbol{\Phi}_{1}^{3}+3 \boldsymbol{D}_{2} \boldsymbol{\Phi}_{1} \boldsymbol{\Phi}_{2}\right)\left[\boldsymbol{Z}_{\lambda d}\left(\boldsymbol{Z}_{\lambda}+\boldsymbol{Z}_{\lambda_{2}}\right) \boldsymbol{Z}_{\beta_{2} d}-2 \boldsymbol{Z}_{\lambda_{2} d} \boldsymbol{Z}_{\lambda_{2}} \boldsymbol{Z}_{\beta_{2} d}\right] \boldsymbol{\Phi}_{1} W \mathbf{1}\right\} \\
& +3\left\{\mathbf { 1 } ^ { \top } ( \boldsymbol { D } _ { 3 } \boldsymbol { \Phi } _ { 1 } ^ { 3 } + 3 \boldsymbol { D } _ { 2 } \boldsymbol { \Phi } _ { 1 } \boldsymbol { \Phi } _ { 2 } ) \left[\boldsymbol{Z}_{\lambda d}\left(\boldsymbol{Z}_{\lambda}+\boldsymbol{Z}_{\lambda_{2}}\right) \boldsymbol{Z}_{\lambda_{2} d}-2 \boldsymbol{Z}_{\lambda_{2} d} \boldsymbol{Z}_{\lambda_{2}} \boldsymbol{Z}_{\lambda_{2} d}\right.\right. \\
& \left.\left.+2\left(\boldsymbol{Z}_{\lambda}-\boldsymbol{Z}_{\lambda_{2}}\right) \odot \boldsymbol{Z}_{\lambda_{2}}^{(2)}\right]\left(\boldsymbol{D}_{3} \boldsymbol{\Phi}_{1}^{3}+3 \boldsymbol{D}_{2} \boldsymbol{\Phi}_{1} \boldsymbol{\Phi}_{2}\right) \mathbf{1}\right\} \\
& A_{12}=12\left\{\mathbf{1}^{\top} \boldsymbol{\Phi}(\boldsymbol{F}+\boldsymbol{G})\left[\boldsymbol{Z}_{\beta d} \boldsymbol{Z}_{\beta} \boldsymbol{Z}_{\beta d}-\boldsymbol{Z}_{\beta_{2} d} \boldsymbol{Z}_{\beta_{2}} \boldsymbol{Z}_{\beta_{2} d}+\boldsymbol{Z}_{\beta}^{(3)}-\boldsymbol{Z}_{\beta_{2}}^{(3)}\right] \boldsymbol{\Phi}(\boldsymbol{F}+\boldsymbol{G}) \mathbf{1}\right\} \\
& +12\left\{\mathbf{1}^{\top}\left(\boldsymbol{D}_{3} \boldsymbol{\Phi}_{1}^{3}+2 \boldsymbol{D}_{2} \boldsymbol{\Phi}_{1} \boldsymbol{\Phi}_{2}\right)\left[\boldsymbol{Z}_{\lambda d} \boldsymbol{Z}_{\lambda} \boldsymbol{Z}_{\lambda d}-\boldsymbol{Z}_{\lambda_{2} d} \boldsymbol{Z}_{\lambda_{2}} \boldsymbol{Z}_{\lambda_{2} d}+\boldsymbol{Z}_{\lambda}^{(3)}-\boldsymbol{Z}_{\lambda_{2}}^{(3)}\right]\right. \\
& \left.\times\left(\boldsymbol{D}_{3} \boldsymbol{\Phi}_{1}^{3}+2 \boldsymbol{D}_{2} \boldsymbol{\Phi}_{1} \boldsymbol{\Phi}_{2}\right) \mathbf{1}\right\}
\end{aligned}
$$




$$
\begin{aligned}
A_{13}=- & 6\left\{\mathbf { 1 } ^ { \top } \boldsymbol { \Phi } ( \boldsymbol { F } + 2 \boldsymbol { G } ) \left[\left(\boldsymbol{Z}_{\beta}^{(2)}-\boldsymbol{Z}_{\beta_{2}}^{(2)}\right) \odot\left(\boldsymbol{Z}_{\beta}+\boldsymbol{Z}_{\beta_{2}}\right)+\left(\boldsymbol{Z}_{\beta d}-3 \boldsymbol{Z}_{\beta_{2} d}\right) \boldsymbol{Z}_{\beta_{2}} \boldsymbol{Z}_{\beta_{2} d}\right.\right. \\
& \left.\left.+\left(\boldsymbol{Z}_{\beta d}+\boldsymbol{Z}_{\beta_{2} d}\right) \boldsymbol{Z}_{\beta} \boldsymbol{Z}_{\beta d}+2 \boldsymbol{Z}_{\beta_{2}}^{(2)} \odot\left(\boldsymbol{Z}_{\beta}-\boldsymbol{Z}_{\beta_{2}}\right)\right] \boldsymbol{\Phi}(\boldsymbol{F}+\boldsymbol{G}) \mathbf{1}\right\} \\
& -6\left\{\mathbf{1}^{\top} \boldsymbol{\Phi}_{1} \boldsymbol{W}\left[\left(\boldsymbol{Z}_{\beta}^{(2)}-\boldsymbol{Z}_{\beta_{2}}^{(2)}\right) \odot\left(\boldsymbol{Z}_{\lambda}+\boldsymbol{Z}_{\lambda_{2}}\right)+2 \boldsymbol{Z}_{\beta_{2}}^{(2)} \odot\left(\boldsymbol{Z}_{\lambda}-\boldsymbol{Z}_{\lambda_{2}}\right)\right] \boldsymbol{\Phi}_{1} \boldsymbol{W} \mathbf{1}\right\} \\
& +6\left\{\mathbf{1}^{\top} \boldsymbol{\Phi}_{1} \boldsymbol{W}\left[\left(\boldsymbol{Z}_{\beta d}+\boldsymbol{Z}_{\beta_{2} d}\right) \boldsymbol{Z}_{\lambda} \boldsymbol{Z}_{\lambda d}+\left(\boldsymbol{Z}_{\beta d}-3 \boldsymbol{Z}_{\beta_{2} d}\right) \boldsymbol{Z}_{\lambda_{2}} \boldsymbol{Z}_{\lambda_{2} d}\right]\left(\boldsymbol{D}_{3} \boldsymbol{\Phi}_{1}^{3}+2 \boldsymbol{D}_{2} \boldsymbol{\Phi}_{1} \boldsymbol{\Phi}_{2}\right) \mathbf{1}\right\} \\
& -6\left\{\mathbf { 1 } ^ { \top } ( \boldsymbol { D } _ { 3 } \boldsymbol { \Phi } _ { 1 } ^ { 3 } + 3 \boldsymbol { D } _ { 2 } \boldsymbol { \Phi } _ { 1 } \boldsymbol { \Phi } _ { 2 } ) \left[\left(\boldsymbol{Z}_{\lambda}^{(2)}-\boldsymbol{Z}_{\lambda_{2}}^{(2)}\right) \odot\left(\boldsymbol{Z}_{\lambda}+\boldsymbol{Z}_{\lambda_{2}}\right)+\left(\boldsymbol{Z}_{\lambda d}-3 \boldsymbol{Z}_{\lambda_{2} d}\right) \boldsymbol{Z}_{\lambda_{2}} \boldsymbol{Z}_{\lambda_{2} d}\right.\right. \\
& \left.\left.\quad+\left(\boldsymbol{Z}_{\lambda d}+\boldsymbol{Z}_{\lambda_{2} d}\right) \boldsymbol{Z}_{\lambda} \boldsymbol{Z}_{\lambda d}+2 \boldsymbol{Z}_{\lambda_{2}}^{(2)} \odot\left(\boldsymbol{Z}_{\lambda}-\boldsymbol{Z}_{\lambda_{2}}\right)\right]\left(\boldsymbol{D}_{3} \boldsymbol{\Phi}_{1}^{3}+2 \boldsymbol{D}_{2} \boldsymbol{\Phi}_{1} \boldsymbol{\Phi}_{2}\right) \mathbf{1}\right\} \\
& \quad \\
A_{14}= & 6\left\{\mathbf{1}^{\top} \boldsymbol{\Phi} \boldsymbol{B}\left(\boldsymbol{Z}_{\beta d}-\boldsymbol{Z}_{\beta_{2} d}\right) \boldsymbol{Z}_{\beta_{2} d} \mathbf{1}\right\}+6\left\{\mathbf{1}^{\top} \boldsymbol{\Phi}_{2} \boldsymbol{W}\left[\left(\boldsymbol{Z}_{\beta d}-\boldsymbol{Z}_{\beta_{2} d}\right) \boldsymbol{Z}_{\lambda_{d}}+\left(\boldsymbol{Z}_{\lambda d}-\boldsymbol{Z}_{\lambda_{2} d}\right) \boldsymbol{Z}_{\beta_{2} d}\right] \mathbf{1}\right\} \\
+ & 6\left\{\mathbf{1}^{\top}\left(\boldsymbol{D}_{4} \boldsymbol{\Phi}_{1}^{4}+6 \boldsymbol{D}_{3} \boldsymbol{\Phi}_{1}^{2} \boldsymbol{\Phi}_{2}+3 \boldsymbol{D}_{2} \boldsymbol{\Phi}_{2}^{2}+4 \boldsymbol{D}_{2} \boldsymbol{\Phi}_{1} \boldsymbol{\Phi}_{3}\right)\left(\boldsymbol{Z}_{\lambda d}-\boldsymbol{Z}_{\lambda_{2} d}\right) \boldsymbol{Z}_{\lambda_{2} d} \mathbf{1}\right\} \\
- & 6\left\{\mathbf{1}^{\top} \boldsymbol{\Phi} \boldsymbol{C}\left(\boldsymbol{Z}_{\beta d}-\boldsymbol{Z}_{\beta_{2} d}\right)\left(3 \boldsymbol{Z}_{\beta_{2} d}+\boldsymbol{Z}_{\beta d}\right) \mathbf{1}\right\} \\
- & 6\left\{\mathbf{1}^{\top}\left(\boldsymbol{D}_{4} \boldsymbol{\Phi}_{1}^{4}+6 \boldsymbol{D}_{3} \boldsymbol{\Phi}_{1}^{2} \boldsymbol{\Phi}_{2}+3 \boldsymbol{D}_{2} \boldsymbol{\Phi}_{2}^{2}+3 \boldsymbol{D}_{2} \boldsymbol{\Phi}_{1} \boldsymbol{\Phi}_{3}\right)\left(\boldsymbol{Z}_{\lambda d}-\boldsymbol{Z}_{\lambda_{2} d}\right)\left(\boldsymbol{Z}_{\lambda d}+3 \boldsymbol{Z}_{\lambda_{2} d}\right) \mathbf{1}\right\} \\
+ & 12\left\{\mathbf{1}^{\top} \boldsymbol{\Phi} \boldsymbol{E}\left(\boldsymbol{Z}_{\beta d}^{(2)}-\boldsymbol{Z}_{\beta_{2} d}^{(2)}\right) \mathbf{1}\right\} \\
+ & 12\left\{\boldsymbol{1}^{\top}\left(\boldsymbol{D}_{4} \boldsymbol{\Phi}_{1}^{4}+5 \boldsymbol{D}_{3} \boldsymbol{\Phi}_{1}^{2} \boldsymbol{\Phi}_{2}+2 \boldsymbol{D}_{2} \boldsymbol{\Phi}_{2}^{2}+2 \boldsymbol{D}_{2} \boldsymbol{\Phi}_{1} \boldsymbol{\Phi}_{3}\right)\left(\boldsymbol{Z}_{\lambda d}^{(2)}-\boldsymbol{Z}_{\lambda_{2} d}^{(2)}\right) \mathbf{1}\right\}
\end{aligned}
$$

$$
A_{2}=A_{21}+A_{22}+A_{23},
$$


em que

$$
\begin{aligned}
& A_{21}=-3\left\{\boldsymbol { 1 } ^ { \top } \boldsymbol { \Phi } ( \boldsymbol { F } + 2 \boldsymbol { G } ) \left[\frac{1}{4}\left(\boldsymbol{Z}_{\beta d}-\boldsymbol{Z}_{\beta_{2} d}\right)\left(\boldsymbol{Z}_{\beta}-\boldsymbol{Z}_{\beta_{2}}\right)\left(3 \boldsymbol{Z}_{\beta d}+\boldsymbol{Z}_{\beta_{2} d}\right)\right.\right. \\
& \left.\left.+\left(\boldsymbol{Z}_{\beta d}-\boldsymbol{Z}_{\beta_{2} d}\right) \boldsymbol{Z}_{\beta_{2}}\left(\boldsymbol{Z}_{\beta d}-\boldsymbol{Z}_{\beta_{2} d}\right)+\frac{1}{2}\left(\boldsymbol{Z}_{\beta}-\boldsymbol{Z}_{\beta_{2}}\right)^{(2)} \odot\left(\boldsymbol{Z}_{\beta}+3 \boldsymbol{Z}_{\beta_{2}}\right)\right] \boldsymbol{\Phi}(\boldsymbol{F}+2 \boldsymbol{G}) \mathbf{1}\right\} \\
& -3\left\{\boldsymbol { 1 } ^ { \top } \boldsymbol { \Phi } _ { 1 } \boldsymbol { W } \left[\frac{1}{4}\left(\boldsymbol{Z}_{\beta d}-\boldsymbol{Z}_{\beta_{2} d}\right)\left(\boldsymbol{Z}_{\lambda}-\boldsymbol{Z}_{\lambda_{2}}\right)\left(3 \boldsymbol{Z}_{\beta d}+\boldsymbol{Z}_{\beta_{2} d}\right)+\left(\boldsymbol{Z}_{\beta d}-\boldsymbol{Z}_{\beta_{2} d}\right) \boldsymbol{Z}_{\lambda_{2}}\left(\boldsymbol{Z}_{\beta d}-\boldsymbol{Z}_{\beta_{2} d}\right)\right.\right. \\
& \left.+\frac{1}{2}\left(\boldsymbol{Z}_{\beta}-\boldsymbol{Z}_{\beta_{2}}\right)^{(2)} \odot\left(\boldsymbol{Z}_{\lambda}+3 \boldsymbol{Z}_{\lambda_{2}}\right)+\left(\boldsymbol{Z}_{\beta}-\boldsymbol{Z}_{\beta_{2}}\right) \odot\left(\boldsymbol{Z}_{\lambda}-\boldsymbol{Z}_{\lambda_{2}}\right) \odot\left(\boldsymbol{Z}_{\beta}+3 \boldsymbol{Z}_{\beta_{2}}\right)\right] \\
& \left.\times \Phi_{1} W 1\right\} \\
& +3\left\{\boldsymbol{1}^{\top} \boldsymbol{\Phi}_{1} \boldsymbol{W}\left(\boldsymbol{Z}_{\beta d}-\boldsymbol{Z}_{\beta_{2} d}\right)\left[\frac{1}{4}\left(\boldsymbol{Z}_{\lambda}-\boldsymbol{Z}_{\lambda_{2}}\right)\left(3 \boldsymbol{Z}_{\lambda d}+\boldsymbol{Z}_{\lambda_{2} d}\right)+\boldsymbol{Z}_{\lambda_{2}}\left(\boldsymbol{Z}_{\lambda d}-\boldsymbol{Z}_{\lambda_{2} d}\right)\right]\right. \\
& \left.\times\left(\boldsymbol{D}_{3} \boldsymbol{\Phi}_{1}^{3}+3 \boldsymbol{D}_{2} \boldsymbol{\Phi}_{1} \boldsymbol{\Phi}_{2}\right) \mathbf{1}\right\} \\
& +3\left\{\boldsymbol { 1 } ^ { \top } ( \boldsymbol { D } _ { 3 } \boldsymbol { \Phi } _ { 1 } ^ { 3 } + 3 \boldsymbol { D } _ { 2 } \boldsymbol { \Phi } _ { 1 } \boldsymbol { \Phi } _ { 2 } ) ( \boldsymbol { Z } _ { \lambda d } - \boldsymbol { Z } _ { \lambda _ { 2 } d } ) \left[\frac{1}{4}\left(\boldsymbol{Z}_{\lambda}-\boldsymbol{Z}_{\lambda_{2}}\right)\left(3 \boldsymbol{Z}_{\beta d}+\boldsymbol{Z}_{\beta_{2} d}\right)\right.\right. \\
& \left.\left.+Z_{\lambda_{2}}\left(Z_{\beta d}-Z_{\beta_{2} d}\right)\right] \Phi_{1} W \mathbf{1}\right\} \\
& -3\left\{\boldsymbol { 1 } ^ { \top } ( \boldsymbol { D } _ { 3 } \boldsymbol { \Phi } _ { 1 } ^ { 3 } + 3 \boldsymbol { D } _ { 2 } \boldsymbol { \Phi } _ { 1 } \boldsymbol { \Phi } _ { 2 } ) \left[\frac{1}{4}\left(\boldsymbol{Z}_{\lambda d}-\boldsymbol{Z}_{\lambda_{2} d}\right)\left(\boldsymbol{Z}_{\lambda}-\boldsymbol{Z}_{\lambda_{2}}\right)\left(3 \boldsymbol{Z}_{\lambda d}+\boldsymbol{Z}_{\lambda_{2} d}\right)\right.\right. \\
& \left.+\left(\boldsymbol{Z}_{\lambda d}-\boldsymbol{Z}_{\lambda_{2} d}\right) \boldsymbol{Z}_{\lambda_{2}}\left(\boldsymbol{Z}_{\lambda d}-\boldsymbol{Z}_{\lambda_{2} d}\right)+\frac{1}{2}\left(\boldsymbol{Z}_{\lambda}-\boldsymbol{Z}_{\lambda_{2}}\right)^{(2)} \odot\left(\boldsymbol{Z}_{\lambda}+3 \boldsymbol{Z}_{\lambda_{2}}\right)\right] \\
& \left.\times\left(\boldsymbol{D}_{3} \boldsymbol{\Phi}_{1}^{3}+3 \boldsymbol{D}_{2} \boldsymbol{\Phi}_{1} \boldsymbol{\Phi}_{2}\right) 1\right\}
\end{aligned}
$$

$$
\begin{aligned}
A_{22}= & 6\left\{\mathbf{1}^{\top} \boldsymbol{\Phi}(\boldsymbol{F}+2 \boldsymbol{G})\left[\left(\boldsymbol{Z}_{\beta}-\boldsymbol{Z}_{\beta_{2}}\right) \odot\left(\boldsymbol{Z}_{\beta}^{(2)}-\boldsymbol{Z}_{\beta_{2}}^{(2)}\right)+\left(\boldsymbol{Z}_{\beta d}-\boldsymbol{Z}_{\beta_{2} d}\right)\left(\boldsymbol{Z}_{\beta} \boldsymbol{Z}_{\beta d}-\boldsymbol{Z}_{\beta_{2}} \boldsymbol{Z}_{\beta_{2} d}\right)\right]\right. \\
& \times \boldsymbol{\Phi}(\boldsymbol{F}+\boldsymbol{G}) \mathbf{1}\} \\
+ & 6\left\{\mathbf{1}^{\top} \boldsymbol{\Phi} \boldsymbol{W}\left[\left(\boldsymbol{Z}_{\lambda}-\boldsymbol{Z}_{\lambda_{2}}\right) \odot\left(\boldsymbol{Z}_{\beta}^{(2)}-\boldsymbol{Z}_{\beta_{2}}^{(2)}\right)\right] \boldsymbol{\Phi}_{1} \boldsymbol{W} \mathbf{1}\right\} \\
+ & 6\left\{\mathbf { 1 } ^ { \top } ( \boldsymbol { D } _ { 3 } \boldsymbol { \Phi } _ { 1 } ^ { 3 } + 3 \boldsymbol { D } _ { 2 } \boldsymbol { \Phi } _ { 1 } \boldsymbol { \Phi } _ { 2 } ) \left[\left(\boldsymbol{Z}_{\lambda}-\boldsymbol{Z}_{\lambda_{2}}\right) \odot\left(\boldsymbol{Z}_{\lambda}^{(2)}-\boldsymbol{Z}_{\lambda_{2}}^{(2)}\right)\right.\right. \\
& \left.\left.+\left(\boldsymbol{Z}_{\lambda d}-\boldsymbol{Z}_{\lambda_{2} d}\right)\left(\boldsymbol{Z}_{\lambda} \boldsymbol{Z}_{\lambda d}-\boldsymbol{Z}_{\lambda_{2}} \boldsymbol{Z}_{\lambda_{2} d}\right)\right]\left(\boldsymbol{D}_{3} \boldsymbol{\Phi}_{1}^{3}+2 \boldsymbol{D}_{2} \boldsymbol{\Phi}_{1} \boldsymbol{\Phi}_{2}\right) \mathbf{1}\right\} \\
-6\left\{\mathbf{1}^{\top} \boldsymbol{\Phi}_{1} \boldsymbol{W}\left(\boldsymbol{Z}_{\beta d}-\boldsymbol{Z}_{\beta_{2} d}\right)\left(\boldsymbol{Z}_{\lambda} \boldsymbol{Z}_{\lambda d}-\boldsymbol{Z}_{\lambda_{2}} \boldsymbol{Z}_{\lambda_{2} d}\right)\left(\boldsymbol{D}_{3} \boldsymbol{\Phi}_{1}^{3}+2 \boldsymbol{D}_{2} \boldsymbol{\Phi}_{1} \boldsymbol{\Phi}_{2}\right) \mathbf{1}\right\} & \\
& \\
A_{23}= & 3\left\{\mathbf{1}^{\top} \boldsymbol{\Phi}(2 \boldsymbol{C}-\boldsymbol{B})\left(\boldsymbol{Z}_{\beta d}-\boldsymbol{Z}_{\beta_{2} d}\right)^{(2)} \mathbf{1}\right\} \\
& +3\left\{\mathbf{1}^{\top}\left(\boldsymbol{D}_{4} \boldsymbol{\Phi}_{1}^{4}+6 \boldsymbol{D}_{3} \boldsymbol{\Phi}_{1}^{2} \boldsymbol{\Phi}_{2}+3 \boldsymbol{D}_{2} \boldsymbol{\Phi}_{2}^{2}+2 \boldsymbol{D}_{2} \boldsymbol{\Phi}_{1} \boldsymbol{\Phi}_{3}\right)\left(\boldsymbol{Z}_{\lambda d}-\boldsymbol{Z}_{\lambda_{2} d}\right)^{(2)} \mathbf{1}\right\}
\end{aligned}
$$


e

$$
\begin{aligned}
A_{3}= & \mathbf{1}^{\top} \boldsymbol{\Phi}(\boldsymbol{F}+2 \boldsymbol{G})\left[\frac{3}{4}\left(\boldsymbol{Z}_{\beta d}-\boldsymbol{Z}_{\beta_{2} d}\right)\left(\boldsymbol{Z}_{\beta}-\boldsymbol{Z}_{\beta_{2}}\right)\left(\boldsymbol{Z}_{\beta d}-\boldsymbol{Z}_{\beta_{2} d}\right)+\frac{1}{2}\left(\boldsymbol{Z}_{\beta}-\boldsymbol{Z}_{\beta_{2}}\right)^{(3)}\right] \boldsymbol{\Phi}(\boldsymbol{F}+2 \boldsymbol{G}) \mathbf{1} \\
+ & \mathbf{1}^{\top} \boldsymbol{\Phi}_{1} \boldsymbol{W}\left[\frac{3}{4}\left(\boldsymbol{Z}_{\beta d}-\boldsymbol{Z}_{\beta_{2} d}\right)\left(\boldsymbol{Z}_{\lambda}-\boldsymbol{Z}_{\lambda_{2}}\right)\left(\boldsymbol{Z}_{\beta d}-\boldsymbol{Z}_{\beta_{2} d}\right)+\frac{3}{2}\left(\boldsymbol{Z}_{\beta}-\boldsymbol{Z}_{\beta_{2}}\right)^{(2)} \odot\left(\boldsymbol{Z}_{\lambda}-\boldsymbol{Z}_{\lambda_{2}}\right)\right] \\
& \quad \times \boldsymbol{\Phi}_{1} \boldsymbol{W} \mathbf{1} \\
- & \frac{3}{2} \mathbf{1}^{\top} \boldsymbol{\Phi}_{1} \boldsymbol{W}\left(\boldsymbol{Z}_{\beta d}-\boldsymbol{Z}_{\beta_{2} d}\right)\left(\boldsymbol{Z}_{\lambda}-\boldsymbol{Z}_{\lambda_{2}}\right)\left(\boldsymbol{Z}_{\lambda d}-\boldsymbol{Z}_{\lambda_{2} d}\right)\left(\boldsymbol{D}_{3} \boldsymbol{\Phi}_{1}^{3}+3 \boldsymbol{D}_{2} \boldsymbol{\Phi}_{1} \boldsymbol{\Phi}_{2}\right) \mathbf{1} \\
+ & \mathbf{1}^{\top}\left(\boldsymbol{D}_{3} \boldsymbol{\Phi}_{1}^{3}+3 \boldsymbol{D}_{2} \boldsymbol{\Phi}_{1} \boldsymbol{\Phi}_{2}\right)\left[\frac{3}{4}\left(\boldsymbol{Z}_{\lambda d}-\boldsymbol{Z}_{\lambda_{2} d}\right)\left(\boldsymbol{Z}_{\lambda}-\boldsymbol{Z}_{\lambda_{2}}\right)\left(\boldsymbol{Z}_{\lambda d}-\boldsymbol{Z}_{\lambda_{2} d}\right)+\frac{1}{2}\left(\boldsymbol{Z}_{\lambda}-\boldsymbol{Z}_{\lambda_{2}}\right)^{(3)}\right] \\
& \quad \times\left(\boldsymbol{D}_{3} \boldsymbol{\Phi}_{1}^{3}+3 \boldsymbol{D}_{2} \boldsymbol{\Phi}_{1} \boldsymbol{\Phi}_{2}\right) \mathbf{1} .
\end{aligned}
$$

Maiores detalhes sobre a dedução destas expressões encontram-se no Apêndice B. Caso haja a presença de parâmetros desconhecidos, estes devem ser substituídos por suas respectivas estimativas de máxima verossimilhanças sob a hipótese nula.

\subsubsection{Hipótese sobre um subconjunto de $\beta$}

Suponha que estamos interessados em testar $\mathcal{H}_{0}: \boldsymbol{\beta}_{1}=\boldsymbol{\beta}_{1}^{(0)}$ contra a hipótese alternativa $\mathcal{H}_{1}: \boldsymbol{\beta}_{1} \neq \boldsymbol{\beta}_{1}^{(0)}$. Para este caso, basta considerar $\boldsymbol{Z}_{\lambda}=\boldsymbol{Z}_{\lambda_{2}}$. Assim, os coeficientes da estatística gradiente aperfeiçoada são dados por:

$$
\begin{aligned}
A_{11}=3 & \left\{\mathbf{1}^{\top} \boldsymbol{\Phi}(\boldsymbol{F}+2 \boldsymbol{G})\left[\boldsymbol{Z}_{\beta d}\left(\boldsymbol{Z}_{\beta}+\boldsymbol{Z}_{\beta_{2}}\right) \boldsymbol{Z}_{\beta_{2} d}-2 \boldsymbol{Z}_{\beta_{2} d} \boldsymbol{Z}_{\beta_{2}} \boldsymbol{Z}_{\beta_{2} d}+2\left(\boldsymbol{Z}_{\beta}-\boldsymbol{Z}_{\beta_{2}}\right) \odot \boldsymbol{Z}_{\beta_{2}}^{(2)}\right]\right. \\
& \times \boldsymbol{\Phi}(\boldsymbol{F}+2 \boldsymbol{G}) \mathbf{1}\} \\
+ & 6\left\{\mathbf{1}^{\top} \boldsymbol{\Phi}_{1} \boldsymbol{W}\left[\left(\boldsymbol{Z}_{\beta d}-\boldsymbol{Z}_{\beta_{2} d}\right) \boldsymbol{Z}_{\lambda} \boldsymbol{Z}_{\beta_{2} d}+2\left(\boldsymbol{Z}_{\beta}-\boldsymbol{Z}_{\beta_{2}}\right) \odot \boldsymbol{Z}_{\beta_{2}} \odot \boldsymbol{Z}_{\lambda_{2}}\right] \boldsymbol{\Phi}_{1} \boldsymbol{W} \mathbf{1}\right. \\
- & 6\left\{\mathbf{1}^{\top} \boldsymbol{\Phi}_{1} \boldsymbol{W}\left(\boldsymbol{Z}_{\beta d}-\boldsymbol{Z}_{\beta_{2} d}\right) \boldsymbol{Z}_{\lambda} \boldsymbol{Z}_{\lambda d}\left(\boldsymbol{D}_{3} \boldsymbol{\Phi}_{1}^{3}+3 \boldsymbol{D}_{2} \boldsymbol{\Phi}_{1} \boldsymbol{\Phi}_{2}\right) \mathbf{1}\right\} \\
A_{12}= & 12\left\{\mathbf{1}^{\top} \boldsymbol{\Phi}(\boldsymbol{F}+\boldsymbol{G})\left[\boldsymbol{Z}_{\beta d} \boldsymbol{Z}_{\beta} \boldsymbol{Z}_{\beta d}-\boldsymbol{Z}_{\beta_{2} d} \boldsymbol{Z}_{\beta_{2}} \boldsymbol{Z}_{\beta_{2} d}+\boldsymbol{Z}_{\beta}^{(3)}-\boldsymbol{Z}_{\beta_{2}}^{(3)}\right] \boldsymbol{\Phi}(\boldsymbol{F}+\boldsymbol{G}) \mathbf{1}\right\}, \\
& \\
A_{13}= & -6\left\{\mathbf { 1 } ^ { \top } \boldsymbol { \Phi } ( \boldsymbol { F } + 2 \boldsymbol { G } ) \left[\left(\boldsymbol{Z}_{\beta}^{(2)}-\boldsymbol{Z}_{\beta_{2}}^{(2)}\right) \odot\left(\boldsymbol{Z}_{\beta}+\boldsymbol{Z}_{\beta_{2}}\right)+\left(\boldsymbol{Z}_{\beta d}-3 \boldsymbol{Z}_{\beta_{2} d}\right) \boldsymbol{Z}_{\beta_{2}} \boldsymbol{Z}_{\beta_{2} d}\right.\right. \\
& \left.\left.+\left(\boldsymbol{Z}_{\beta d}+\boldsymbol{Z}_{\beta_{2} d}\right) \boldsymbol{Z}_{\beta} \boldsymbol{Z}_{\beta d}+2 \boldsymbol{Z}_{\beta_{2}}^{(2)} \odot\left(\boldsymbol{Z}_{\beta}-\boldsymbol{Z}_{\beta_{2}}\right)\right] \boldsymbol{\Phi}(\boldsymbol{F}+\boldsymbol{G}) \mathbf{1}\right\} \\
& -12\left\{\mathbf{1}^{\top} \boldsymbol{\Phi}_{1} \boldsymbol{W}\left[\left(\boldsymbol{Z}_{\beta}^{(2)}-\boldsymbol{Z}_{\beta_{2}}^{(2)}\right) \odot \boldsymbol{Z}_{\lambda}\right] \boldsymbol{\Phi}_{1} \boldsymbol{W} \mathbf{1}\right\} \\
& +12\left\{\mathbf{1}^{\top} \boldsymbol{\Phi}_{1} \boldsymbol{W}\left[\left(\boldsymbol{Z}_{\beta d}-\boldsymbol{Z}_{\beta_{2} d}\right) \boldsymbol{Z}_{\lambda} \boldsymbol{Z}_{\lambda d}\right]\left(\boldsymbol{D}_{3} \boldsymbol{\Phi}_{1}^{3}+2 \boldsymbol{D}_{2} \boldsymbol{\Phi}_{1} \boldsymbol{\Phi}_{2}\right) \mathbf{1}\right\}
\end{aligned}
$$




$$
\begin{gathered}
A_{14}=6\left\{\mathbf{1}^{\top} \boldsymbol{\Phi} \boldsymbol{B}\left(\boldsymbol{Z}_{\beta d}-\boldsymbol{Z}_{\beta_{2} d}\right) \boldsymbol{Z}_{\beta_{2} d} \mathbf{1}\right\}+6\left\{\mathbf{1}^{\top} \boldsymbol{\Phi}_{2} \boldsymbol{W}\left(\boldsymbol{Z}_{\beta d}-\boldsymbol{Z}_{\beta_{2} d}\right) \boldsymbol{Z}_{\lambda d} \mathbf{1}\right\} \\
-6\left\{\mathbf{1}^{\top} \boldsymbol{\Phi} \boldsymbol{C}\left(\boldsymbol{Z}_{\beta d}-\boldsymbol{Z}_{\beta_{2} d}\right)\left(3 \boldsymbol{Z}_{\beta_{2} d}+\boldsymbol{Z}_{\beta d}\right) \mathbf{1}\right\} \\
+12\left\{\mathbf{1}^{\top} \boldsymbol{\Phi} \boldsymbol{E}\left(\boldsymbol{Z}_{\beta d}^{(2)}-\boldsymbol{Z}_{\beta_{2} d}^{(2)}\right) \mathbf{1}\right\}
\end{gathered}
$$

\subsubsection{Hipótese sobre um subconjunto de $\lambda$}

Suponha que estamos interessados em testar $\mathcal{H}_{0}: \boldsymbol{\lambda}_{1}=\boldsymbol{\lambda}_{1}^{(0)}$ contra a hipótese alternativa $\mathcal{H}_{0}: \boldsymbol{\lambda}_{1} \neq \boldsymbol{\lambda}_{1}^{(0)}$. Para este caso, basta considerar $\boldsymbol{Z}_{\beta}=\boldsymbol{Z}_{\beta_{2}}$. Assim, os coeficientes da estatística gradiente aperfeiçoada são dados por:

$$
\begin{aligned}
A_{11}= & 3\left\{\mathbf{1}^{\top} \boldsymbol{\Phi}_{1} \boldsymbol{W}\left[\boldsymbol{Z}_{\beta d}\left(\boldsymbol{Z}_{\lambda}-\boldsymbol{Z}_{\lambda_{2}}\right) \boldsymbol{Z}_{\beta d}+2\left(\boldsymbol{Z}_{\lambda}-\boldsymbol{Z}_{\lambda_{2}}\right) \odot \boldsymbol{Z}_{\beta}^{(2)}\right] \boldsymbol{\Phi}_{1} \boldsymbol{W} \mathbf{1}\right\} \\
- & 3\left\{\mathbf{1}^{\top} \boldsymbol{\Phi}_{1} \boldsymbol{W}\left[\boldsymbol{Z}_{\beta d}\left(\boldsymbol{Z}_{\lambda}-\boldsymbol{Z}_{\lambda_{2}}\right) \boldsymbol{Z}_{\lambda_{2} d}\right]\left(\boldsymbol{D}_{3} \boldsymbol{\Phi}_{1}^{3}+3 \boldsymbol{D}_{2} \boldsymbol{\Phi}_{1} \boldsymbol{\Phi}_{2}\right) \mathbf{1}\right\} \\
- & 3\left\{\mathbf{1}^{\top}\left(\boldsymbol{D}_{3} \boldsymbol{\Phi}_{1}^{3}+3 \boldsymbol{D}_{2} \boldsymbol{\Phi}_{1} \boldsymbol{\Phi}_{2}\right)\left[\boldsymbol{Z}_{\lambda d}\left(\boldsymbol{Z}_{\lambda}+\boldsymbol{Z}_{\lambda_{2}}\right) \boldsymbol{Z}_{\beta d}-2 \boldsymbol{Z}_{\lambda_{2} d} \boldsymbol{Z}_{\lambda_{2}} \boldsymbol{Z}_{\beta d}\right] \boldsymbol{\Phi}_{1} \boldsymbol{W} \mathbf{1}\right\} \\
+ & 3\left\{\mathbf{1}^{\top}\left(\boldsymbol{D}_{3} \boldsymbol{\Phi}_{1}^{3}+3 \boldsymbol{D}_{2} \boldsymbol{\Phi}_{1} \boldsymbol{\Phi}_{2}\right)\left[\boldsymbol{Z}_{\lambda d}\left(\boldsymbol{Z}_{\lambda}+\boldsymbol{Z}_{\lambda_{2}}\right) \boldsymbol{Z}_{\lambda_{2} d}-2 \boldsymbol{Z}_{\lambda_{2} d} \boldsymbol{Z}_{\lambda_{2}} \boldsymbol{Z}_{\lambda_{2} d}+2\left(\boldsymbol{Z}_{\lambda}-\boldsymbol{Z}_{\lambda_{2}}\right) \odot \boldsymbol{Z}_{\lambda_{2}}^{(2)}\right]\right. \\
& \left.\times\left(\boldsymbol{D}_{3} \boldsymbol{\Phi}_{1}^{3}+3 \boldsymbol{D}_{2} \boldsymbol{\Phi}_{1} \boldsymbol{\Phi}_{2}\right) \mathbf{1}\right\} \\
& \\
& \left.\quad \times\left(\boldsymbol{D}_{3} \boldsymbol{\Phi}_{1}^{3}+2 \boldsymbol{D}_{2} \boldsymbol{\Phi}_{1} \boldsymbol{\Phi}_{2}\right) \mathbf{1}\right\}
\end{aligned}
$$




$$
\begin{aligned}
& A_{13}=-12\left\{\mathbf{1}^{\top} \boldsymbol{\Phi}_{1} \boldsymbol{W}\left[\boldsymbol{Z}_{\beta}^{(2)} \odot\left(\boldsymbol{Z}_{\lambda}-\boldsymbol{Z}_{\lambda_{2}}\right)\right] \boldsymbol{\Phi}_{1} \boldsymbol{W} \mathbf{1}\right\} \\
& +12\left\{\mathbf{1}^{\top} \boldsymbol{\Phi}_{1} \boldsymbol{W}\left[\boldsymbol{Z}_{\beta d}\left(\boldsymbol{Z}_{\lambda} \boldsymbol{Z}_{\lambda d}-\boldsymbol{Z}_{\lambda_{2}} \boldsymbol{Z}_{\lambda_{2} d}\right)\right]\left(\boldsymbol{D}_{3} \boldsymbol{\Phi}_{1}^{3}+2 \boldsymbol{D}_{2} \boldsymbol{\Phi}_{1} \boldsymbol{\Phi}_{2}\right) \mathbf{1}\right\} \\
& -6\left\{\mathbf { 1 } ^ { \top } ( \boldsymbol { D } _ { 3 } \boldsymbol { \Phi } _ { 1 } ^ { 3 } + 3 \boldsymbol { D } _ { 2 } \boldsymbol { \Phi } _ { 1 } \boldsymbol { \Phi } _ { 2 } ) \left[\left(\boldsymbol{Z}_{\lambda}^{(2)}-\boldsymbol{Z}_{\lambda_{2}}^{(2)}\right) \odot\left(\boldsymbol{Z}_{\lambda}+\boldsymbol{Z}_{\lambda_{2}}\right)+\left(\boldsymbol{Z}_{\lambda d}-3 \boldsymbol{Z}_{\lambda_{2} d}\right) \boldsymbol{Z}_{\lambda_{2}} \boldsymbol{Z}_{\lambda_{2} d}\right.\right. \\
& \left.\left.+\left(\boldsymbol{Z}_{\lambda d}+\boldsymbol{Z}_{\lambda_{2} d}\right) \boldsymbol{Z}_{\lambda} \boldsymbol{Z}_{\lambda d}+2 \boldsymbol{Z}_{\lambda_{2}}^{(2)} \odot\left(\boldsymbol{Z}_{\lambda}-\boldsymbol{Z}_{\lambda_{2}}\right)\right]\left(\boldsymbol{D}_{3} \boldsymbol{\Phi}_{1}^{3}+2 \boldsymbol{D}_{2} \boldsymbol{\Phi}_{1} \boldsymbol{\Phi}_{2}\right) \mathbf{1}\right\}, \\
& A_{14}=6\left\{\mathbf{1}^{\top} \boldsymbol{\Phi}_{2} \boldsymbol{W}\left(\boldsymbol{Z}_{\lambda d}-\boldsymbol{Z}_{\lambda_{2} d}\right) \boldsymbol{Z}_{\beta_{d}} \mathbf{1}\right\} \\
& +6\left\{\mathbf{1}^{\top}\left(\boldsymbol{D}_{4} \boldsymbol{\Phi}_{1}^{4}+6 \boldsymbol{D}_{3} \boldsymbol{\Phi}_{1}^{2} \boldsymbol{\Phi}_{2}+3 \boldsymbol{D}_{2} \boldsymbol{\Phi}_{2}^{2}+4 \boldsymbol{D}_{2} \boldsymbol{\Phi}_{1} \boldsymbol{\Phi}_{3}\right)\left(\boldsymbol{Z}_{\lambda d}-\boldsymbol{Z}_{\lambda_{2} d}\right) \boldsymbol{Z}_{\lambda_{2} d} \mathbf{1}\right\} \\
& -6\left\{\mathbf{1}^{\top}\left(\boldsymbol{D}_{4} \boldsymbol{\Phi}_{1}^{4}+6 \boldsymbol{D}_{3} \boldsymbol{\Phi}_{1}^{2} \boldsymbol{\Phi}_{2}+3 \boldsymbol{D}_{2} \boldsymbol{\Phi}_{2}^{2}+3 \boldsymbol{D}_{2} \boldsymbol{\Phi}_{1} \boldsymbol{\Phi}_{3}\right)\left(\boldsymbol{Z}_{\lambda d}-\boldsymbol{Z}_{\lambda_{2} d}\right)\left(\boldsymbol{Z}_{\lambda d}+3 \boldsymbol{Z}_{\lambda_{2} d}\right) \mathbf{1}\right\} \\
& +12\left\{\mathbf{1}^{\top}\left(\boldsymbol{D}_{4} \boldsymbol{\Phi}_{1}^{4}+5 \boldsymbol{D}_{3} \boldsymbol{\Phi}_{1}^{2} \boldsymbol{\Phi}_{2}+2 \boldsymbol{D}_{2} \boldsymbol{\Phi}_{2}^{2}+2 \boldsymbol{D}_{2} \boldsymbol{\Phi}_{1} \boldsymbol{\Phi}_{3}\right)\left(\boldsymbol{Z}_{\lambda d}^{(2)}-\boldsymbol{Z}_{\lambda_{2} d}^{(2)}\right) \mathbf{1}\right\}, \\
& A_{21}=3\left\{\mathbf{1}^{\top}\left(\boldsymbol{D}_{3} \boldsymbol{\Phi}_{1}^{3}+3 \boldsymbol{D}_{2} \boldsymbol{\Phi}_{1} \boldsymbol{\Phi}_{2}\right)\left(\boldsymbol{Z}_{\lambda d}-\boldsymbol{Z}_{\lambda_{2} d}\right)\left(\boldsymbol{Z}_{\lambda}-\boldsymbol{Z}_{\lambda_{2}}\right) \boldsymbol{Z}_{\beta d} \boldsymbol{\Phi}_{1} \boldsymbol{W} \mathbf{1}\right\} \\
& -3\left\{\boldsymbol { 1 } ^ { \top } ( \boldsymbol { D } _ { 3 } \boldsymbol { \Phi } _ { 1 } ^ { 3 } + 3 \boldsymbol { D } _ { 2 } \boldsymbol { \Phi } _ { 1 } \boldsymbol { \Phi } _ { 2 } ) \left[\frac{1}{4}\left(\boldsymbol{Z}_{\lambda d}-\boldsymbol{Z}_{\lambda_{2} d}\right)\left(\boldsymbol{Z}_{\lambda}-\boldsymbol{Z}_{\lambda_{2}}\right)\left(3 \boldsymbol{Z}_{\lambda d}+\boldsymbol{Z}_{\lambda_{2} d}\right)\right.\right. \\
& \left.+\left(\boldsymbol{Z}_{\lambda d}-\boldsymbol{Z}_{\lambda_{2} d}\right) \boldsymbol{Z}_{\lambda_{2}}\left(\boldsymbol{Z}_{\lambda d}-\boldsymbol{Z}_{\lambda_{2} d}\right)+\frac{1}{2}\left(\boldsymbol{Z}_{\lambda}-\boldsymbol{Z}_{\lambda_{2}}\right)^{(2)} \odot\left(\boldsymbol{Z}_{\lambda}+3 \boldsymbol{Z}_{\lambda_{2}}\right)\right] \\
& \left.\times\left(\boldsymbol{D}_{3} \boldsymbol{\Phi}_{1}^{3}+3 \boldsymbol{D}_{2} \boldsymbol{\Phi}_{1} \boldsymbol{\Phi}_{2}\right) \mathbf{1}\right\} \\
& A_{22}=6\left\{\mathbf { 1 } ^ { \top } ( \boldsymbol { D } _ { 3 } \boldsymbol { \Phi } _ { 1 } ^ { 3 } + 3 \boldsymbol { D } _ { 2 } \boldsymbol { \Phi } _ { 1 } \boldsymbol { \Phi } _ { 2 } ) \left[\left(\boldsymbol{Z}_{\lambda}-\boldsymbol{Z}_{\lambda_{2}}\right) \odot\left(\boldsymbol{Z}_{\lambda}^{(2)}-\boldsymbol{Z}_{\lambda_{2}}^{(2)}\right)\right.\right. \\
& \left.\left.+\left(\boldsymbol{Z}_{\lambda d}-\boldsymbol{Z}_{\lambda_{2} d}\right)\left(\boldsymbol{Z}_{\lambda} \boldsymbol{Z}_{\lambda d}-\boldsymbol{Z}_{\lambda_{2}} \boldsymbol{Z}_{\lambda_{2} d}\right)\right]\left(\boldsymbol{D}_{3} \boldsymbol{\Phi}_{1}^{3}+2 \boldsymbol{D}_{2} \boldsymbol{\Phi}_{1} \boldsymbol{\Phi}_{2}\right) \mathbf{1}\right\}, \\
& A_{23}=\left\{\mathbf{1}^{\top}\left(\boldsymbol{D}_{4} \boldsymbol{\Phi}_{1}^{4}+6 \boldsymbol{D}_{3} \boldsymbol{\Phi}_{1}^{2} \boldsymbol{\Phi}_{2}+3 \boldsymbol{D}_{2} \boldsymbol{\Phi}_{2}^{2}+2 \boldsymbol{D}_{2} \boldsymbol{\Phi}_{1} \boldsymbol{\Phi}_{3}\right)\left(\boldsymbol{Z}_{\lambda d}-\boldsymbol{Z}_{\lambda_{2} d}\right)^{(2)} \mathbf{1}\right\}
\end{aligned}
$$

e

$$
\begin{aligned}
A_{3}= & \mathbf{1}^{\top}\left(\boldsymbol{D}_{3} \boldsymbol{\Phi}_{1}^{3}+3 \boldsymbol{D}_{2} \boldsymbol{\Phi}_{1} \boldsymbol{\Phi}_{2}\right)\left[\frac{3}{4}\left(\boldsymbol{Z}_{\lambda d}-\boldsymbol{Z}_{\lambda_{2} d}\right)\left(\boldsymbol{Z}_{\lambda}-\boldsymbol{Z}_{\lambda_{2}}\right)\left(\boldsymbol{Z}_{\lambda d}-\boldsymbol{Z}_{\lambda_{2} d}\right)+\frac{1}{2}\left(\boldsymbol{Z}_{\lambda}-\boldsymbol{Z}_{\lambda_{2}}\right)^{(3)}\right] \\
& \times\left(\boldsymbol{D}_{3} \boldsymbol{\Phi}_{1}^{3}+3 \boldsymbol{D}_{2} \boldsymbol{\Phi}_{1} \boldsymbol{\Phi}_{2}\right) \mathbf{1}
\end{aligned}
$$

\subsection{Resultados numéricos}

Nesta seção, nosso objetivo é comparar, via simulação, os desempenhos de vários testes baseados nas estatísticas da razão de verossimilhanças, escore, Wald e gradiente usuais e suas versões aperfeiçoadas.

Comparamos o desempenho de treze testes: teste da razão de verossimilhanças usual 
$(R V)$, teste da razão de verossimilhanças ajustada via ajuste de Skovgaard $\left(R V_{a}\right)^{1}$, teste da razão de verossimilhanças corrigida via correção de Bartlett $\left(R V_{b}\right)$, teste escore usual $(S R)$, teste escore corrigido via correção tipo-Bartlett $\left(S R_{b}\right)$, teste de Wald usual $(W)$, teste de Wald modificado $\left(W_{2}\right)$, teste gradiente usual $(T)$, teste gradiente corrigido via correção tipoBartlett $\left(T_{b}\right)$, e os testes da razão de verossimilhanças, escore, Wald e gradiente bootstrap ( $R V_{\text {boot }}, S R_{\text {boot }}, W_{\text {boot }}$ e $T_{\text {boot }}$, respectivamente).

Iremos analisar os desempenhos dos testes cujo interesse é testar os efeitos na média e na dispersão separadamente. Os desempenhos são avaliados em função da proximidade da probabilidade de rejeição da hipótese nula, sendo esta verdadeira (probabilidade do erro tipo I), aos respectivos níveis nominais dos testes. Avaliamos também o poder dos testes aperfeiçoados em estudo.

As simulações realizadas foram baseadas nos modelos de regressão gama e normal inversa com componentes sistemáticas definidas por

$$
\mu_{\ell}=\beta_{1}+\beta_{2} x_{\ell 2}+\ldots+\beta_{p} x_{\ell p} \quad \text { e } \quad \log \left(\phi_{\ell}\right)=\lambda_{1}+\lambda_{2} s_{\ell 2}+\ldots+\lambda_{q} s_{\ell q}
$$

em que as covariáveis $x_{2}, \ldots, x_{p}$ e $s_{2}, \ldots, s_{q}$ foram obtidas a partir da distribuição $\mathcal{U}(1,2)$ de forma independente. Estas covariáveis foram mantidas constantes durante as simulações. Todos os parâmetros, exceto aqueles fixados na hipótese nula, foram definidos iguais a um.

Todas as simulações foram realizadas utilizando a linguagem de programação matricial ox (Doornik, 2006). A maximização do logaritmo da função de verossimilhança com respeito a $\boldsymbol{\beta}$ e $\boldsymbol{\lambda}$ foi feita através do método quasi-Newton BFGS (Nocedal e Wright, 2006) com primeiras derivadas analíticas. A quantidade de réplicas de Monte Carlo e bootstrap foram fixadas em 5000 e 500, respectivamente, e foram considerados os seguintes níveis nominais: $\alpha=10 \%, 5 \%$ e $1 \%$.

Para cada tamanho da amostra e cada nível nominal considerado, calculamos as taxas de rejeição de cada teste, isto é, estimamos, via simulação, $P\left(R V>z_{\alpha}\right), P\left(R V_{a}>z_{\alpha}\right)$, $P\left(R V_{b}>z_{\alpha}\right), P\left(S R>z_{\alpha}\right), P\left(S R_{a}>z_{\alpha}\right), P\left(W>z_{\alpha}\right), P\left(W_{2}>z_{\alpha}\right), P\left(T>z_{\alpha}\right), P\left(T_{b}>z_{\alpha}\right)$ em que $z_{\alpha}$ é o quantil $(1-\alpha)$ da distribuição $\chi^{2}$ de referência. Em outras palavras, calculamos para cada estatística, a porcentagem de vezes em que a hipótese nula foi rejeitada.

Para os testes baseados nas estatísticas $R V_{\text {boot }}, S R_{\text {boot }}, W_{\text {boot }}$ e $T_{\text {boot }}$, as taxas de rejeição são obtidas das probabilidades: $P\left(R V>\widehat{q}_{(1-\alpha)}\right), P\left(S R>\widehat{q}_{(1-\alpha)}\right), P\left(W>\widehat{q}_{(1-\alpha)}\right)$ e $P(T>$ $\left.\widehat{q}_{(1-\alpha)}\right)$ em que $\widehat{q}_{(1-\alpha)}$ é o quantil bootstrap $(1-\alpha)$ que é estimado através do procedimento descrito na Seção 2.5.

Para as simulações de poder, as taxas de rejeição foram obtidas sob diferentes hipóteses alternativas. Adicionalmente, na avaliação dos testes $R V, R V_{\mathrm{a}}, R V_{\mathrm{b}}, S R, S R_{\mathrm{b}}, W, W_{2}, T$ e $T_{\mathrm{b}}$, utilizou-se os valores críticos estimados obtidos a partir das simulações de tamanho desses testes ao invés dos valores tabelados da distribuição qui-quadrado de referência.

\footnotetext{
${ }^{1}$ Expressão (5) dada em Ferrari e Cysneiros (2008).
} 


\subsubsection{Modelo gama}

Os resultados de simulação apresentados aqui baseiam-se no modelo de regressão gama com componentes sistemáticas dadas em (3.9).

Na Tabela 3.1 apresentamos as taxas de rejeição considerando a hipótese $\mathcal{H}_{0}: \beta_{p}=0$, $n=30, q=2$ e diferentes valores de $p$. Variamos $p$ para analisar o efeito do número de covariáveis presentes no modelo nos diversos testes. Observe que os testes da razão de verossimilhanças $(R V)$ e Wald $(W)$ são extremamente liberais, rejeitando a hipótese nula com mais frequência que o esperado com base no nível nominal, para qualquer valor de $p$. Os testes escore $(S R)$ e gradiente $(T)$ também são liberais em quase todos os casos, mas possuem um tamanho bem menos distorcido que os testes da razão de verossimilhanças e Wald em todos os casos. Por exemplo, quando $p=6$ e $\alpha=5 \%$ as taxas de rejeição dos testes foram $11,08 \%(R V), 7,68 \%(S R), 15,02 \%(W)$ e $9,32 \%(T)$. Os testes $W_{2}$ e $T_{\mathrm{b}}$ possuem desempenhos superiores aos testes $W$ e $T$, respectivamente, principalmente quando o número de parâmetros $p$ aumenta. Constatamos ainda que $W_{2}$ é ligeiramente melhor que $R V$. Podemos notar também que o impacto do número de parâmetros é bem menos acentuado nos testes corrigidos $R V_{\mathrm{a}}, R V_{\mathrm{b}}, S R_{\mathrm{b}}$ e $T_{\mathrm{b}}$. Para os testes $R V_{\mathrm{a}}$ e $R V_{\mathrm{b}}$, o ajuste de Skovgaard e o fator de correção de Bartlett, respectivamente, fizeram com que as taxas de rejeição ficassem mais próximas dos níveis nominais considerados. Por exemplo, para $p=5$ e $\alpha=10 \%$, o teste $R V$ apresentou taxa de rejeição $17,18 \%$, enquanto suas versões modificadas apresentaram taxas $12,06 \%\left(R V_{\mathrm{a}}\right)$ e $12,72 \%\left(R V_{\mathrm{b}}\right)$. Os testes bootstrap, em geral, apresentaram melhor desempenho que os demais testes, mas são parecidos entre si.

Na Tabela 3.2 fixamos a hipótese nula em $\mathcal{H}_{0}: \beta_{p}=0, p=2, n=30$ e diferentes valores de $q$. Os testes que tiveram suas taxas de rejeição mais afetadas pelo aumento do número de parâmetros da dispersão foram os testes $W, R V, T$ e $W_{2}$. Os demais testes mostraram desempenhos razoáveis, sendo que os testes $R V_{\mathrm{a}}$ e $R V_{\mathrm{b}}$ apresentaram tamanhos empíricos mais instáveis em relação aos respectivos níveis nominais. A taxa de rejeição do teste Wald para $q=5$ e $\alpha=5 \%$ foi $19,56 \%$, ou seja, quase o quádruplo do nível nominal considerado, as demais taxas de rejeição dos testes $R V, R V_{\mathrm{a}}, R V_{\mathrm{b}}, S R, S R_{\mathrm{b}}, W_{2}, T, T_{\mathrm{b}}$, $R V_{\text {boot }}, S R_{\text {boot }}, W_{\text {boot }}$ e $T_{\text {boot }}$ foram $11,56 \%, 7,32 \%, 6,64 \%, 5,28 \%, 4,86 \%, 11,26 \%, 9,12 \%$, $4,96 \%, 5,24 \%, 5,42 \%, 5,26 \%$ e $5,72 \%$, respectivamente. As demais conclusões anteriores obtidas da Tabela 3.1 também se verificam aqui.

Na Tabela 3.3, consideramos a hipótese nula em $\mathcal{H}_{0}: \beta_{p}=0, p=3, q=3$ e variamos o tamanho da amostra em 20,30 e 40. Observamos que conforme o tamanho da amostra aumenta, as taxas de rejeição de todos os testes aproximam-se dos respectivos níveis nominais. Além disto, percebemos que os testes $S R_{\mathrm{b}}, T_{\mathrm{b}}$ e os bootstrap apresentaram taxas de rejeição próximas aos correspondentes níveis nominais para todos os valores de $n$. Vale ressaltar aqui que mesmo para $n=40$, os testes baseados nas estatísticas $R V, W, W_{2}$ e $T$ são ainda liberais. Por exemplo, para $\alpha=10 \%$, as taxas de rejeição dos respectivos testes são $14,02 \%(R V)$, 
$16,96 \%(W), 12,32 \%\left(W_{2}\right)$ e $12,86 \%(T)$, respectivamente. As demais conclusões anteriores adquiridas a partir das Tabelas 3.1 e 3.2 também podem ser constatadas aqui.

Na Tabela 3.4, tomamos a hipótese nula $\mathcal{H}_{0}: \lambda_{2}=\ldots=\lambda_{q}=0, q=3, p=2 \mathrm{e}$ diversificamos o tamanho da amostra em 20,30 e 40. Percebemos que as taxas de rejeição de todos os testes aproximam-se dos respectivos níveis nominais, à medida que o tamanho da amostra cresce. Observamos que os testes $R V, W, T$ e $W_{2}$ apresentam altas distorções de tamanhos, sendo a distorção de $W$ a mais acentuada. Notamos também que os testes $S R, S R_{\mathrm{b}}$ e $T_{\mathrm{b}}$ mostraram-se conservativos. Verificamos ainda que o teste $R V_{\mathrm{a}}$ é ligeiramente melhor que o teste $R V_{\mathrm{b}}$. Por fim constatamos que as taxas do teste gradiente corrigido $\left(T_{\mathrm{b}}\right)$ encontram-se bem mais próximas dos níveis nominais que aquelas apresentadas pelo teste gradiente $(T)$. A título de exemplo, para $n=20$, as taxas de rejeição para os níveis $\alpha=10 \%$ e $\alpha=5 \%$ são, respectivamente, $17,70 \%$ e $9,68 \%$ para $T$ e $10,30 \%$ e $4,14 \%$ para $T_{\mathrm{b}}$. Os testes bootstrap obtiveram um desempenho satisfatório.

Na Tabela 3.5, fixamos a hipótese nula $\mathcal{H}_{0}: \lambda_{2}=\ldots=\lambda_{q}=0$ e $p=1$ e variamos o tamanho da amostra em 20,30 e 40 e $q$ em 2,3 e 4. É possível observar que o teste Wald $(W)$ é bastante liberal, à medida que o número de parâmetros aumenta. Os testes da razão de verossimilhanças $(R V)$, Wald modificado $\left(W_{2}\right)$ e gradiente $(T)$ também são liberais, mas possuem um tamanho muito menos distorcido que o teste $W$. Podemos perceber também que os testes escore $(S R)$ e gradiente corrigido $\left(T_{\mathrm{b}}\right)$ são conservativos, sendo este último em quase todos os casos. Notamos ainda que conforme o tamanho da amostra aumenta, os testes aproximam-se dos respectivos níveis nominais. Dentre os testes da razão de verossimilhanças modificadas, o teste $R V_{\mathrm{a}}$ apresentou resultados ligeiramente melhores. Por fim, os testes bootstrap e $S R_{\mathrm{b}}$ proporcionaram uma considerável redução na distorção de tamanho.

Na Tabela 3.6 apresentamos os resultados de simulação para o poder. Fixamos $n=30$, $p=1, q=2$ e $\alpha=10 \%$ e consideramos a hipótese $\mathcal{H}_{1}: \lambda_{2}=\epsilon$, em que diferentes valores de $\epsilon$ foram considerados. Desconsideramos os testes usuais, a saber, $R V, S R, W$ e $T$. Como esperado, o poder dos testes aumenta à medida que $\epsilon$ aumenta. Podemos observar que os poderes de todos os testes são bem semelhantes.

Na Figura 3.1 apresentamos o gráfico dos quantis assintóticos versus as discrepâncias relativas dos quantis. A discrepância relativa é definida como a diferença entre o quantil exato (estimado via simulação) e o quantil assintótico dividido por este último. Quanto mais próxima de zero estiver a discrepância, melhor a aproximação da distribuição exata sob a hipótese nula da estatística de teste pela distribuição limite $\chi^{2}$. Consideramos a hipótese nula $\mathcal{H}_{0}: \beta_{3}=0, p=q=3$ e $n=40$, ou seja, os quantis assintóticos são obtidos da distribuição $\chi_{1}^{2}$. A figura confirma a tendência do teste Wald $(W)$ em ser demasiadamente liberal. Os testes da razão de verossimilhanças $(R V)$, gradiente $(T)$ e Wald modificado $\left(W_{2}\right)$ também são liberais mas bem menos que $W$. Os demais testes rejeitam a hipótese nula com menos frequência, comparados com o nível nominal. Nota-se que a distribuição do teste $T_{\mathrm{b}}$ é muito mais próxima da distribuição de referência do que a distribuição de $T$. A melhor 
concordância entre os quantis exatos e assintóticos é obtida por $T_{\mathrm{b}}$.

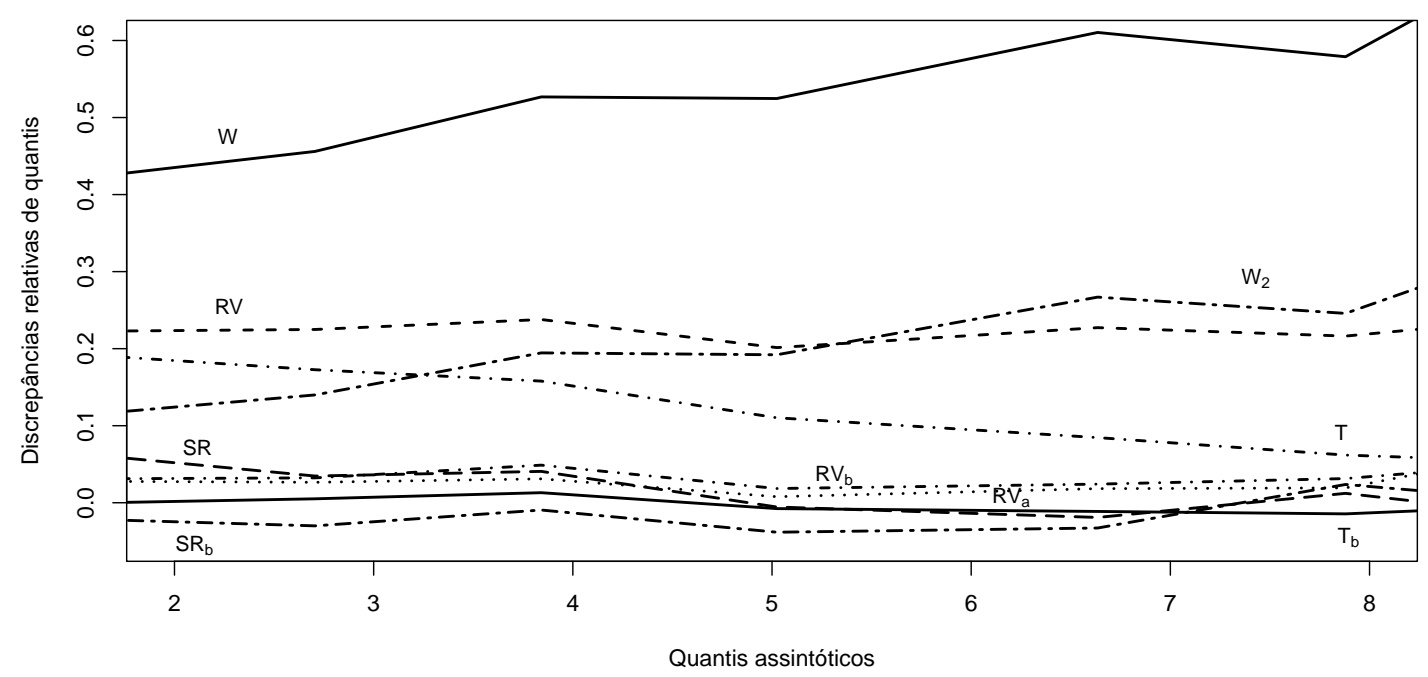

Figura 3.1: Discrepâncias relativas de quantis - modelo gama com $n=40, p=3$ e $q=3$.

\subsubsection{Modelo normal inversa}

Os resultados de simulação apresentados aqui baseiam-se no modelo de regressão normal inversa com componentes sistemáticas dadas em (3.9).

Na Tabela 3.7 apresentamos as taxas de rejeição considerando a hipótese $\mathcal{H}_{0}: \beta_{p}=0, n=$ $30, q=2$ e diferentes valores de $p$. Variamos $p$ para analisar o efeito do número de covariáveis presentes no modelo nos diversos testes. Note que os testes da razão de verossimilhanças usual $(R V)$, ajustada $\left(R V_{\mathrm{a}}\right)$ e corrigida Bartlett $\left(R V_{\mathrm{b}}\right)$, os testes de Wald usual $(W)$ e modificado $\left(W_{2}\right)$ e o teste gradiente $(T)$ são extremamente liberais, principalmente quando o número de parâmetros aumenta. O teste escore $(S R)$ também é liberal em quase todos casos, porém possui um tamanho bem menos distorcido que os testes citados anteriormente em todas as situações. Por exemplo, para $p=5$ e $\alpha=5 \%$ as taxas de rejeição dos testes foram $17,28 \%(R V), 10,12 \%\left(R V_{\mathrm{a}}\right), 9,82 \%\left(R V_{\mathrm{b}}\right), 6,18 \%(S R), 28,98 \%(W), 16,58 \%\left(W_{2}\right)$ e $9,22 \%$ $(T)$. Podemos observar que a influência do número de parâmetros é bem menos acentuada nos testes assintóticos $S R, S R_{\mathrm{b}}$ e $T_{\mathrm{b}}$. Para o teste $T_{\mathrm{b}}$, o fator de correção tipo-Bartlett fez com que as taxas de rejeição ficassem mais próximas dos níveis nominais considerados, sendo que produziu taxas claramente melhores em relação a sua versão usual. Nota-se ainda que os testes $S R$ e $S R_{\mathrm{b}}$ possuem desempenhos semelhantes. Os testes bootstrap, em geral, possibilitaram uma significativa redução na distorção do tamanho, especialmente para os testes da razão de verossimilhanças e Wald, em que suas versões modificadas ficaram a desejar.

Na Tabela 3.8 fixamos a hipótese nula em $\mathcal{H}_{0}: \beta_{p}=0, p=2, n=30$ e diferentes 


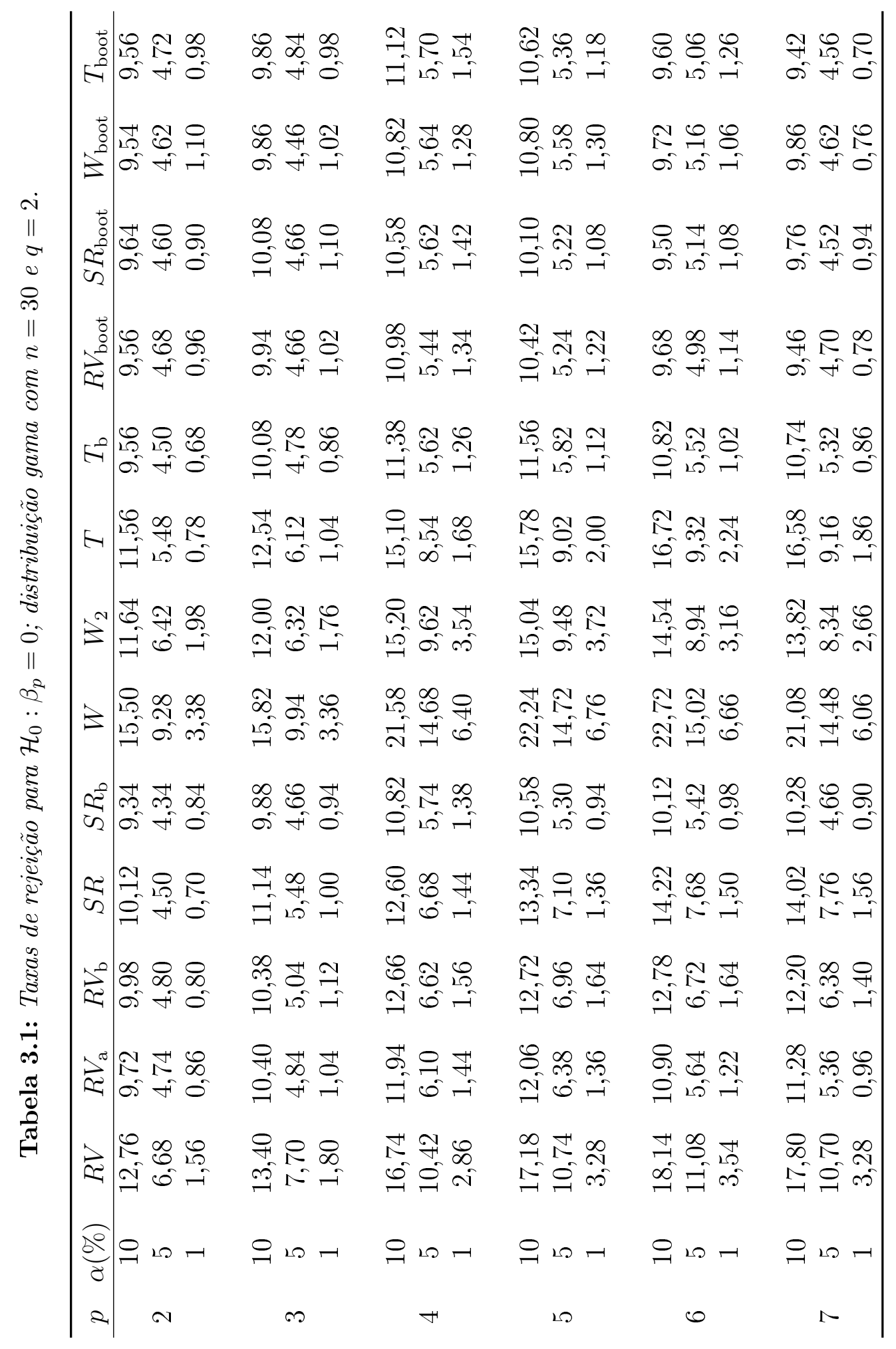


Tabela 3.2: Taxas de rejeição para $\mathcal{H}_{0}: \beta_{p}=0$; distribuição gama com $n=30$ e $p=2$.

\begin{tabular}{|c|c|c|c|c|c|c|}
\hline \multirow[b]{2}{*}{ Estatística } & \multicolumn{3}{|c|}{$q=2$} & \multicolumn{3}{|c|}{$q=3$} \\
\hline & $\alpha=10 \%$ & $\alpha=5 \%$ & $\alpha=1 \%$ & $\alpha=10 \%$ & $\alpha=5 \%$ & $\alpha=1 \%$ \\
\hline$R V$ & 12,76 & 6,68 & 1,56 & 15,42 & 9,12 & 2,54 \\
\hline$R V_{\mathrm{a}}$ & 9,72 & 4,74 & 0,86 & 11,52 & 5,80 & 1,48 \\
\hline$R V_{\mathrm{b}}$ & 9,98 & 4,80 & 0,80 & 12,04 & 6,02 & 1,44 \\
\hline$S R$ & 10,12 & 4,50 & 0,70 & 11,44 & 5,68 & 0,98 \\
\hline$S R_{\mathrm{b}}$ & 9,34 & 4,34 & 0,84 & 10,26 & 5,26 & 1,04 \\
\hline$W$ & 15,50 & 9,28 & 3,38 & 20,28 & 13,86 & 5,80 \\
\hline$W_{2}$ & 11,64 & 6,42 & 1,98 & 14,76 & 8,60 & 3,18 \\
\hline$T$ & 11,56 & 5,48 & 0,78 & 14,40 & 7,54 & 1,56 \\
\hline$T_{\mathrm{b}}$ & 9,56 & 4,50 & 0,68 & 10,88 & 5,36 & 1,14 \\
\hline$R V_{\text {boot }}$ & 9,56 & 4,68 & 0,96 & 10,56 & 5,44 & 1,38 \\
\hline$S R_{\text {boot }}$ & 9,64 & 4,60 & 0,90 & 10,62 & 5,48 & 1,28 \\
\hline$W_{\text {boot }}$ & 9,54 & 4,62 & 1,10 & 10,78 & 5,36 & 1,32 \\
\hline \multirow[t]{2}{*}{$T_{\text {boot }}$} & 9,56 & 4,72 & 0,98 & 10,82 & 5,68 & 1,52 \\
\hline & \multicolumn{3}{|c|}{$q=4$} & \multicolumn{3}{|c|}{$q=5$} \\
\hline Estatística & $\alpha=10 \%$ & $\alpha=5 \%$ & $\alpha=1 \%$ & $\alpha=10 \%$ & $\alpha=5 \%$ & $\alpha=1 \%$ \\
\hline$R V$ & 16,70 & 9,98 & 2,84 & 18,38 & 11,56 & 3,56 \\
\hline$R V_{\mathrm{a}}$ & 11,80 & 6,18 & 1,26 & 13,74 & 7,32 & 1,96 \\
\hline$R V_{\mathrm{b}}$ & 11,46 & 6,00 & 1,26 & 12,68 & 6,64 & 1,40 \\
\hline$S R$ & 10,50 & 5,26 & 0,70 & 11,22 & 5,28 & 0,74 \\
\hline$S R_{\mathrm{b}}$ & 9,70 & 5,02 & 0,84 & 9,98 & 4,86 & 0,84 \\
\hline$W$ & 23,90 & 16,74 & 8,22 & 26,98 & 19,56 & 10,08 \\
\hline$W_{2}$ & 15,54 & 9,72 & 3,66 & 17,28 & 11,26 & 4,68 \\
\hline$T$ & 14,86 & 7,88 & 1,18 & 16,34 & 9,12 & 1,76 \\
\hline$T_{\mathrm{b}}$ & 9,98 & 4,88 & 0,78 & 10,38 & 4,96 & 0,92 \\
\hline$R V_{\text {boot }}$ & 9,88 & 4,94 & 1,08 & 10,10 & 5,24 & 1,22 \\
\hline$S R_{\text {boot }}$ & 9,76 & 5,20 & 1,10 & 10,50 & 5,42 & 1,24 \\
\hline$W_{\text {boot }}$ & 9,72 & 4,80 & 1,22 & 10,08 & 5,26 & 1,08 \\
\hline$T_{\text {boot }}$ & 9,96 & 5,26 & 1,14 & 10,66 & 5,72 & 1,34 \\
\hline
\end{tabular}




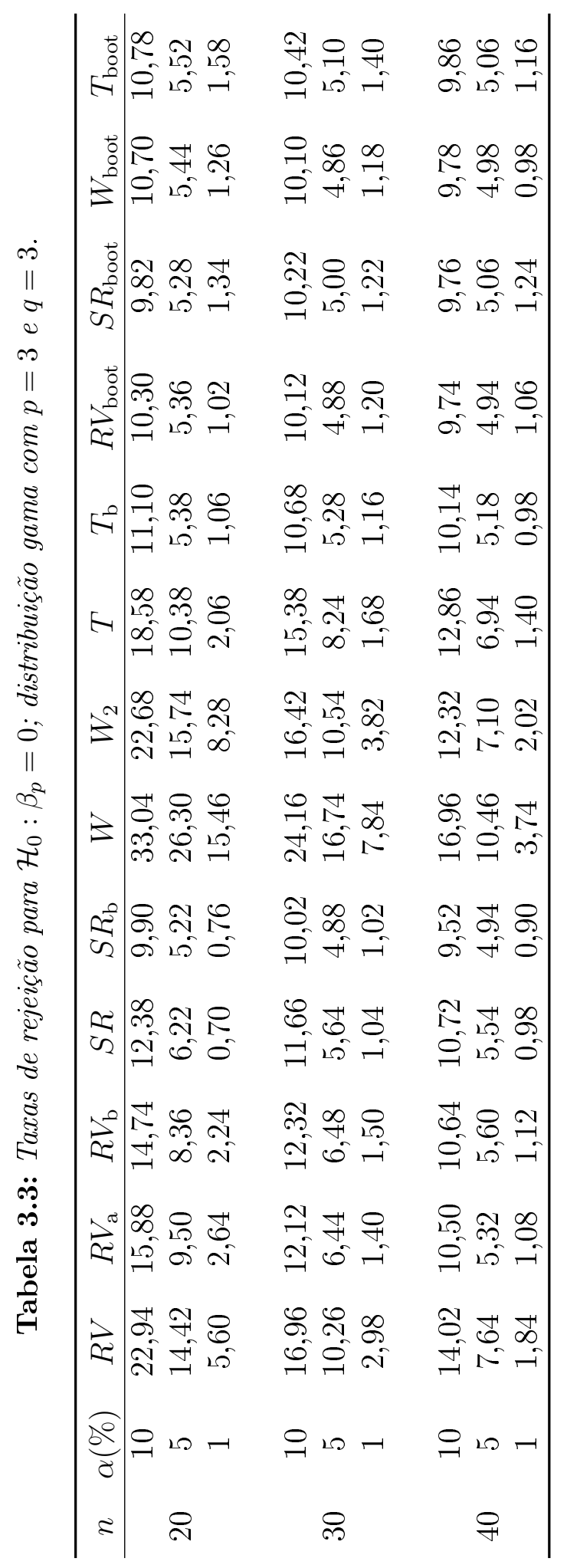




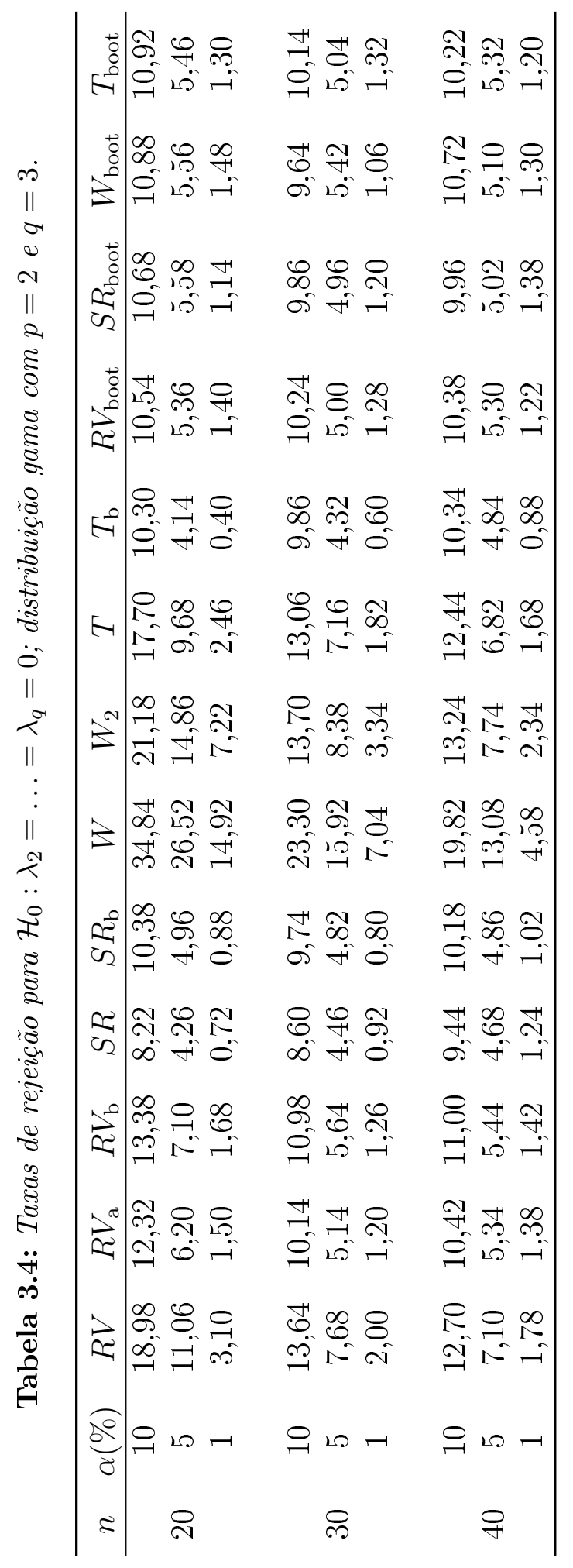




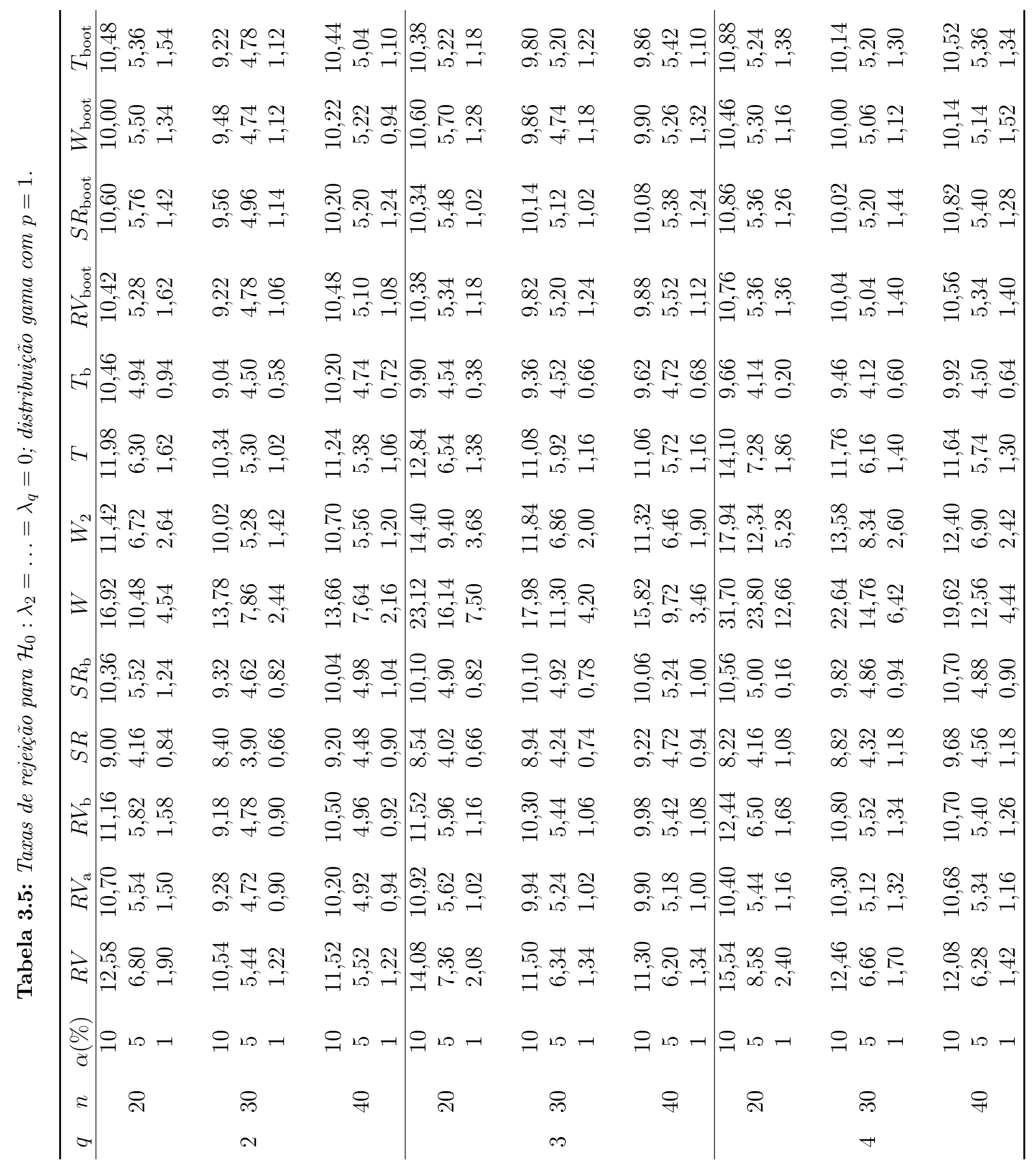


Tabela 3.6: Poder para $\mathcal{H}_{1}: \lambda_{2}=\epsilon ; n=30, p=1, q=2, \alpha=10 \%$ e distribuição gama.

\begin{tabular}{c|ccccccccc}
\hline$\epsilon$ & $R V_{\mathrm{a}}$ & $R V_{\mathrm{b}}$ & $S R_{\mathrm{b}}$ & $W_{2}$ & $T_{\mathrm{b}}$ & $R V_{\text {boot }}$ & $S R_{\text {boot }}$ & $W_{\text {boot }}$ & $T_{\text {boot }}$ \\
\hline 0,5 & 16,06 & 16,32 & 15,84 & 15,56 & 16,04 & 15,14 & 15,16 & 14,82 & 15,10 \\
1,5 & 42,76 & 43,16 & 41,40 & 41,00 & 42,84 & 41,38 & 40,18 & 40,08 & 41,46 \\
2,5 & 75,78 & 76,06 & 72,66 & 75,52 & 75,84 & 74,80 & 71,64 & 74,54 & 74,90 \\
3,5 & 94,06 & 94,12 & 91,30 & 94,62 & 94,06 & 93,82 & 90,84 & 94,40 & 93,78 \\
\hline
\end{tabular}

valores de $q$. Podemos observar que os testes assintóticos $S R, S R_{\mathrm{b}}$ e $T_{\mathrm{b}}$ e os testes bootstrap praticamente não são afetados com o aumento de $q$, o mesmo não acontecendo com os demais testes. Percebemos que os testes escore corrigido $\left(S R_{\mathrm{b}}\right)$ e usual $(S R)$ são conservativos, sendo este último em quase todos os casos. Note também que os testes $R V, W, W_{2}$ e $T$ são liberais, sendo o teste de Wald $(W)$ o que possui uma maior distorção de tamanho.

Na Tabela 3.9, consideramos a hipótese nula em $\mathcal{H}_{0}: \beta_{p}=0, p=3, q=3$ e variamos o tamanho da amostra em 20,30 e 40. Observamos que conforme o tamanho da amostra aumenta, as taxas de rejeição de todos os testes aproximam-se dos respectivos níveis nominais. O teste Wald usual $(W)$ apresentou taxas de rejeição muito acima dos níveis nominais considerados. Por exemplo, quando $n=20$ e $\alpha=5 \%$, a taxa de rejeição do teste de Wald foi de $19,78 \%$, isto é, quase o quádruplo do nível nominal fixado. Percebemos que as taxas de rejeição do teste gradiente foram atenuadas pela correção tipo-Bartlett. Como ilustração, para $n=30$ e $\alpha=5 \%$, a taxa do teste gradiente foi $7,60 \%$ enquanto que a taxa do teste gradiente corrigido foi $5,30 \%$. Notamos ainda que os testes $S R, S R_{\mathrm{b}}, T_{\mathrm{b}}$ e os bootstrap apresentaram taxas de rejeição próximas aos correspondentes níveis nominais para todos os valores de $n$.

Na Tabela 3.10, tomamos a hipótese nula $\mathcal{H}_{0}: \lambda_{2}=\ldots=\lambda_{q}=0, q=3, p=2 \mathrm{e}$ diversificamos o tamanho da amostra em 20,30 e 40. Percebemos que as taxas de rejeição de todos os testes aproximam-se dos respectivos níveis nominais, à medida que o tamanho da amostra cresce. Observamos que os testes $R V, W, T$ e $W_{2}$ apresentam altas distorções de tamanhos, sendo a distorção de $W$ a mais acentuada. Notamos também que os testes $S R$ e $T_{\mathrm{b}}$ mostraram-se conservativos. Verificamos ainda que os testes $R V_{\mathrm{a}}$ e $R V_{\mathrm{b}}$ apresentaram comportamentos semelhantes. Os testes bootstrap possuem, em geral, desempenho superior aos demais testes.

Na Tabela 3.11, fixamos a hipótese nula $\mathcal{H}_{0}: \lambda_{2}=\ldots=\lambda_{q}=0$ e $p=1$ e variamos o tamanho da amostra em 20,30 e 40 e $q$ em 2,3 e 4. É possível notar que conforme o tamanho da amostra aumenta, os testes aproximam-se dos respectivos níveis nominais. Observamos também que o teste Wald $(W)$ possui altas distorções de tamanho à medida que o número de parâmetros aumenta. Os testes $R V, W_{2}$ e $T$ são liberais, mas menos distorcidos do que $W$. Podemos perceber ainda que os testes escore $(S R)$ e gradiente corrigido $\left(T_{\mathrm{b}}\right)$ são conservativos, sendo este último em quase todos os casos. Os testes da razão de verossimilhanças 
modificadas $\left(R V_{\mathrm{a}}\right.$ e $\left.R V_{\mathrm{b}}\right)$ apresentaram resultados semelhantes. Por fim, os testes bootstrap e $S R_{\mathrm{b}}$ proporcionaram uma considerável redução na distorção de tamanho.

Na Tabela 3.12 apresentamos os resultados de simulação para o poder. Fixamos $n=30$, $p=1, q=2$ e $\alpha=10 \%$ e consideramos a hipótese $\mathcal{H}_{1}: \lambda_{2}=\epsilon$, em que diferentes valores de $\epsilon$ foram considerados. Desconsideramos apenas os testes usuais, a saber, $R V, S R, W$ e $T$. Como esperado, o poder dos testes aumenta à medida que $\epsilon$ aumenta. Podemos observar que os poderes dos testes $R V_{\mathrm{b}}$ e $T_{\mathrm{b}}$ são similares, o mesmo acontecendo com $R V_{\text {boot }}$ e $T_{\text {boot }}$. Notamos ainda que o teste $R V_{\mathrm{a}}$ é ligeiramente mais poderoso que os demais.

Na Figura 3.2 apresentamos o gráfico dos quantis assintóticos versus as discrepâncias relativas dos quantis. A discrepância relativa foi definida na Seção 3.4.1. Consideramos a hipótese nula $\mathcal{H}_{0}: \beta_{3}=0, p=q=3$ e $n=40$, ou seja, os quantis assintóticos são obtidos da distribuição $\chi_{1}^{2}$. A figura confirma a tendência do teste Wald $(W)$ em ser demasiadamente liberal. Os testes da razão de verossimilhanças $(R V)$, gradiente $(T)$ e Wald modificado $\left(W_{2}\right)$ também são liberais mas bem menos que $W$. Os demais testes rejeitam a hipótese nula com menos frequência, comparados ao nível nominal. Nota-se que as menores discrepâncias são dadas pelos testes $S R, S R_{\mathrm{b}}$ e $T_{\mathrm{b}}$. 


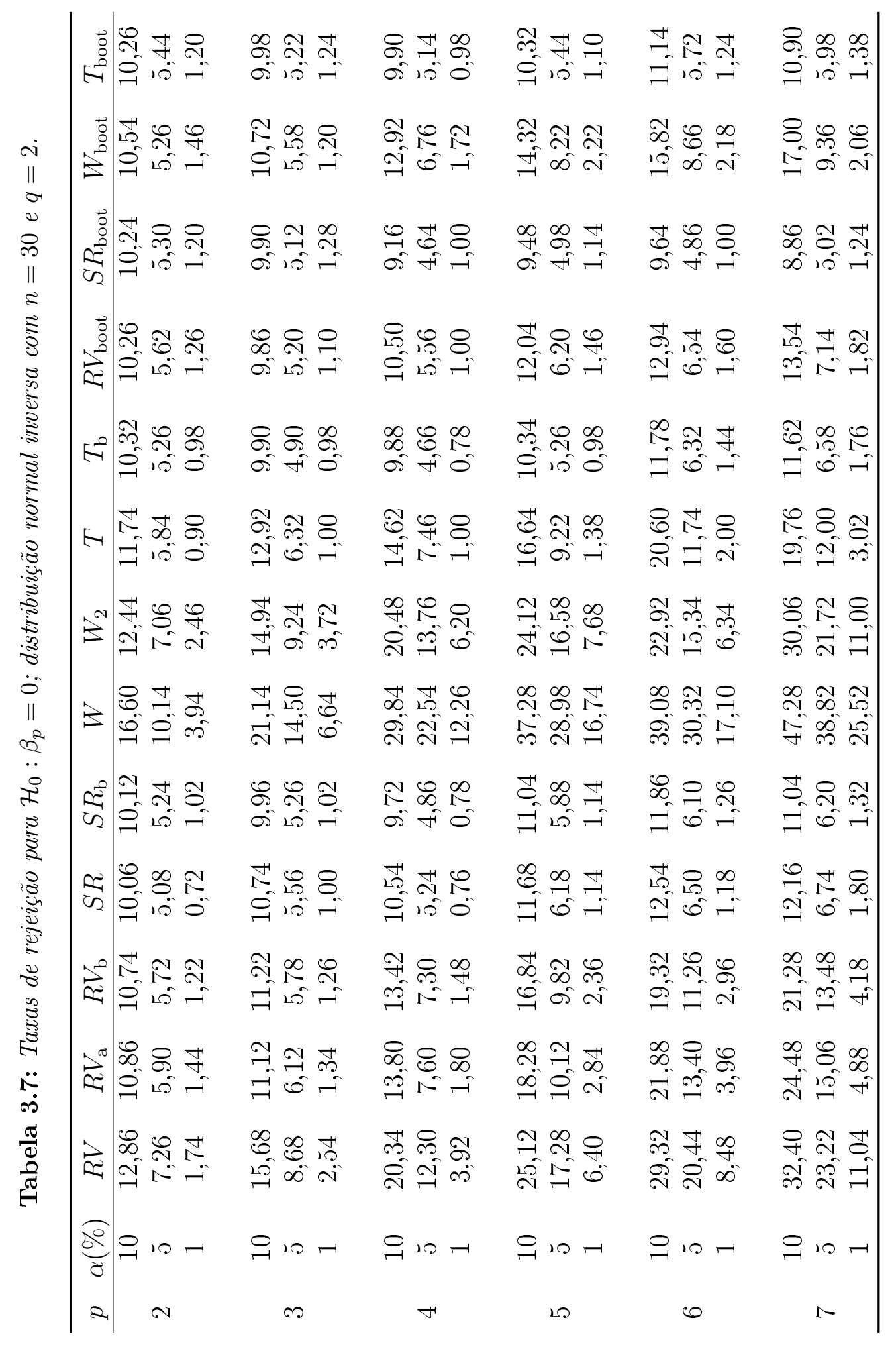


Tabela 3.8: Taxas de rejeição para $\mathcal{H}_{0}: \beta_{p}=0$; distribuição normal inversa com $n=30$ e $p=2$.

\begin{tabular}{|c|c|c|c|c|c|c|}
\hline \multirow[b]{2}{*}{ Estatística } & \multicolumn{3}{|c|}{$q=2$} & \multicolumn{3}{|c|}{$q=3$} \\
\hline & $\alpha=10 \%$ & $\alpha=5 \%$ & $\alpha=1 \%$ & $\alpha=10 \%$ & $\alpha=5 \%$ & $\alpha=1 \%$ \\
\hline$R V$ & 12,96 & 6,58 & 1,70 & 14,26 & 7,72 & 2,16 \\
\hline$R V_{\mathrm{a}}$ & 10,40 & 5,22 & 1,18 & 11,18 & 5,78 & 1,30 \\
\hline$R V_{\mathrm{b}}$ & 10,32 & 4,98 & 1,04 & 10,72 & 5,50 & 1,20 \\
\hline$S R$ & 9,62 & 4,36 & 0,58 & 9,64 & 4,44 & 0,68 \\
\hline$S R_{\mathrm{b}}$ & 9,72 & 4,66 & 0,78 & 9,60 & 4,66 & 0,90 \\
\hline$W$ & 16,68 & 9,92 & 3,54 & 19,36 & 12,18 & 5,18 \\
\hline$W_{2}$ & 11,80 & 6,90 & 2,26 & 12,98 & 7,68 & 2,40 \\
\hline$T$ & 11,36 & 5,36 & 0,82 & 12,84 & 6,58 & 1,26 \\
\hline$T_{\mathrm{b}}$ & 9,86 & 4,72 & 0,88 & 10,08 & 5,08 & 1,06 \\
\hline$R V_{\text {boot }}$ & 10,32 & 4,98 & 1,26 & 10,02 & 5,14 & 1,20 \\
\hline$S R_{\text {boot }}$ & 10,16 & 4,90 & 0,92 & 10,00 & 4,92 & 0,98 \\
\hline$W_{\text {boot }}$ & 10,16 & 5,22 & 1,24 & 10,02 & 5,04 & 1,30 \\
\hline$T_{\text {boot }}$ & 10,36 & 4,96 & 1,18 & 10,20 & 5,14 & 1,18 \\
\hline \multirow[b]{2}{*}{ Estatística } & \multicolumn{3}{|c|}{$q=4$} & \multicolumn{3}{|c|}{$q=5$} \\
\hline & $\alpha=10 \%$ & $\alpha=5 \%$ & $\alpha=1 \%$ & $\alpha=10 \%$ & $\alpha=5 \%$ & $\alpha=1 \%$ \\
\hline$R V$ & 16,24 & 9,38 & 2,90 & 18,52 & 11,46 & 3,84 \\
\hline$R V_{\mathrm{a}}$ & 12,28 & 6,52 & 1,58 & 14,22 & 8,22 & 2,44 \\
\hline$R V_{\mathrm{b}}$ & 11,76 & 5,98 & 1,36 & 12,34 & 6,88 & 1,60 \\
\hline$S R$ & 10,10 & 4,68 & 0,76 & 10,04 & 4,58 & 0,60 \\
\hline$S R_{\mathrm{b}}$ & 9,66 & 4,68 & 0,98 & 9,46 & 4,46 & 0,78 \\
\hline$W$ & 23,40 & 16,74 & 7,56 & 28,60 & 20,74 & 11,60 \\
\hline$W_{2}$ & 15,98 & 9,16 & 3,68 & 17,56 & 11,92 & 5,34 \\
\hline$T$ & 14,70 & 7,82 & 1,56 & 15,88 & 9,14 & 1,68 \\
\hline$T_{\mathrm{b}}$ & 10,22 & 4,94 & 1,04 & 10,14 & 5,14 & 0,84 \\
\hline$R V_{\text {boot }}$ & 10,08 & 5,04 & 1,26 & 10,04 & 5,20 & 1,08 \\
\hline$S R_{\text {boot }}$ & 10,44 & 5,20 & 1,26 & 10,04 & 5,04 & 1,16 \\
\hline$W_{\text {boot }}$ & 9,84 & 4,94 & 1,26 & 9,88 & 4,90 & 1,16 \\
\hline$T_{\text {boot }}$ & 10,46 & 5,22 & 1,40 & 10,52 & 5,94 & 1,32 \\
\hline
\end{tabular}




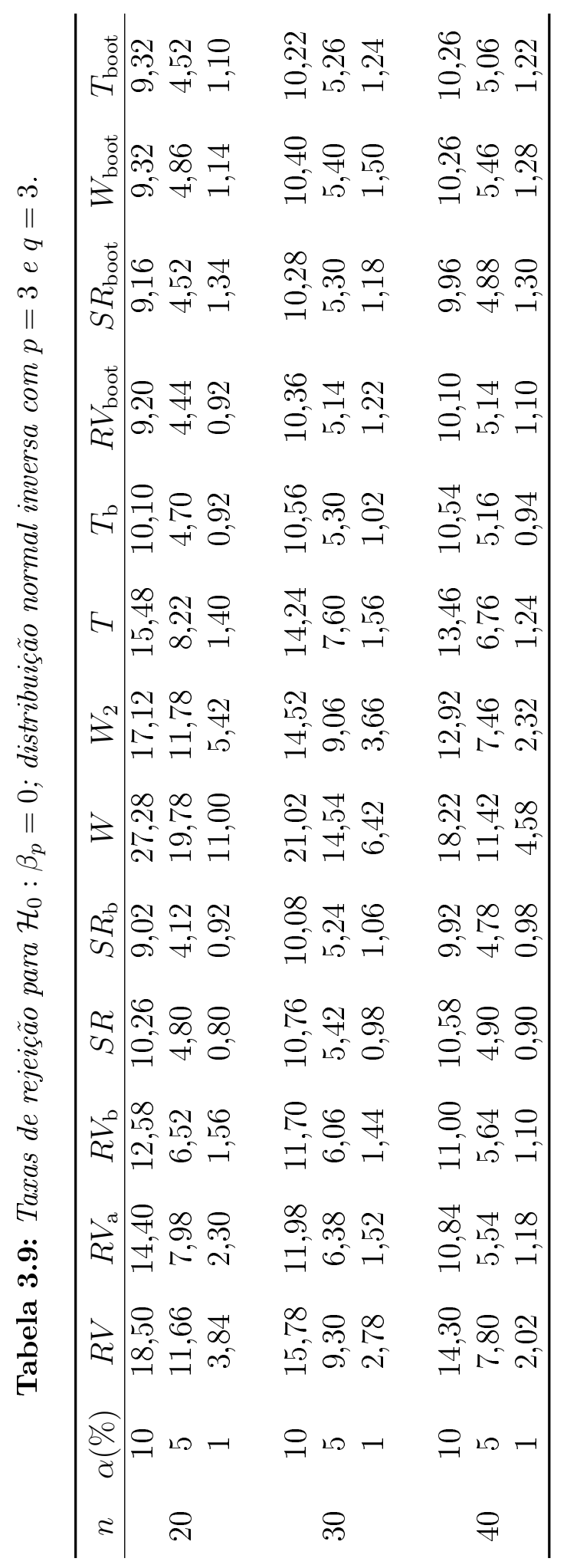




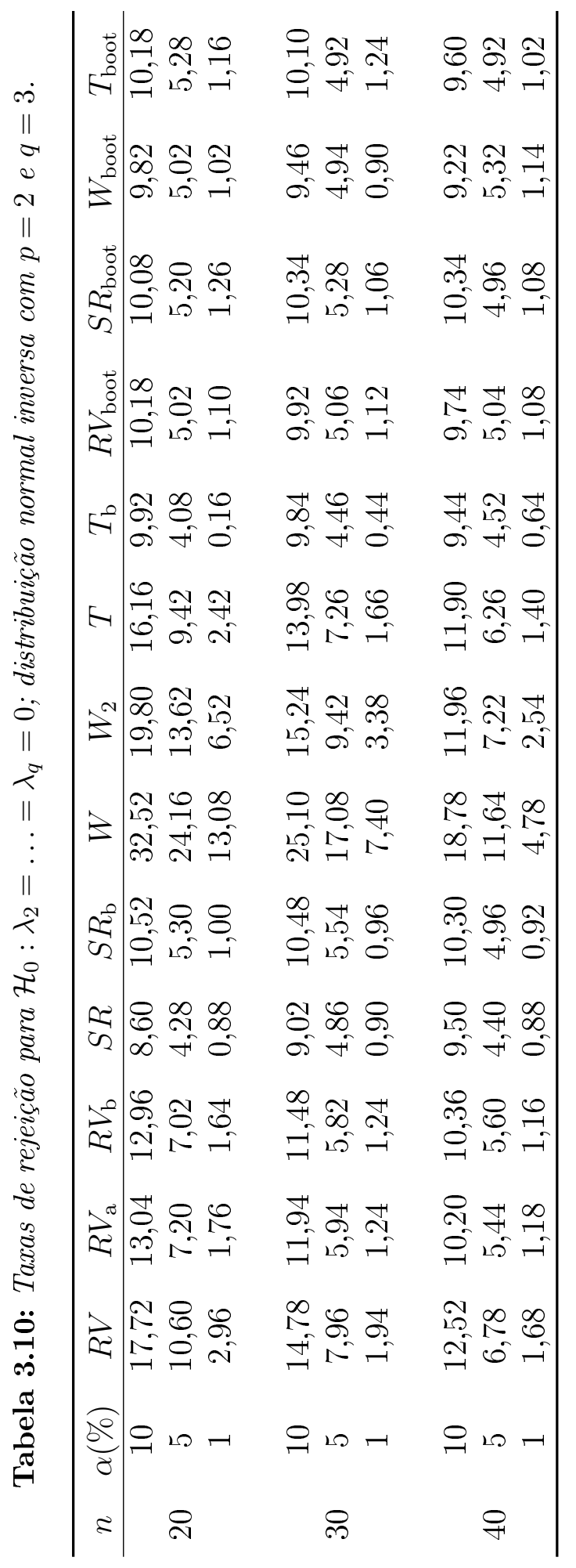




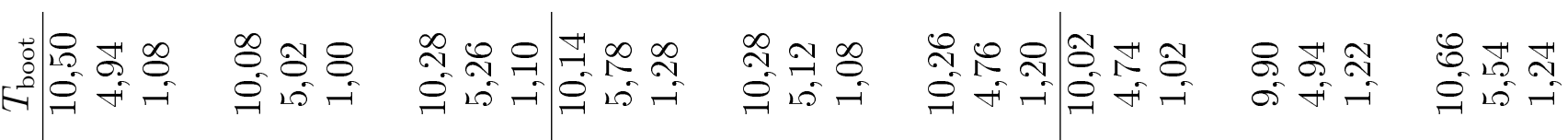

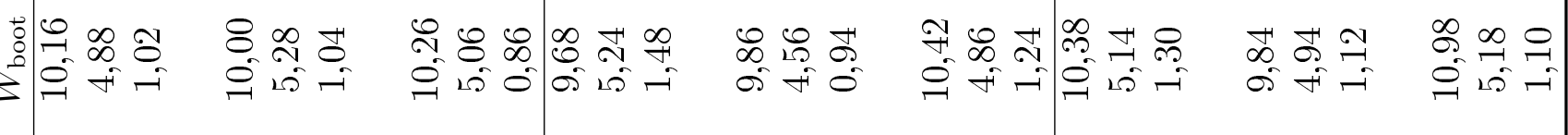

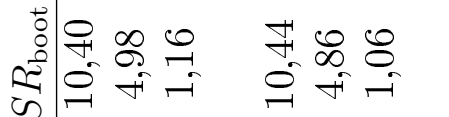

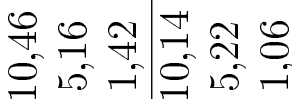

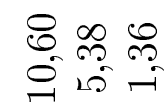

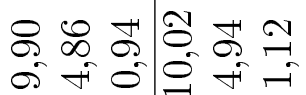

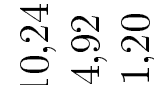

路 ํำ

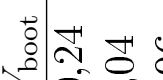

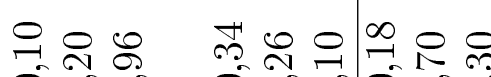

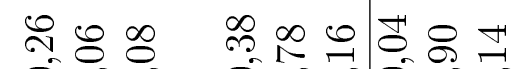

กิ 8 ก in 15

मी $\rightarrow$ or

$\because \propto \exists$ 位

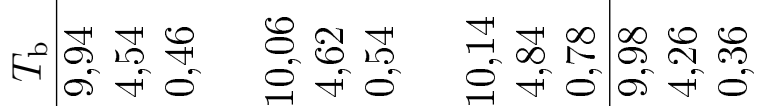

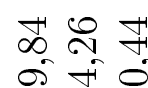

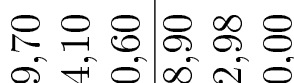

$\infty$. $015-7$

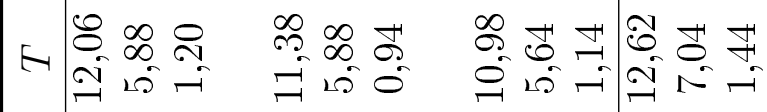

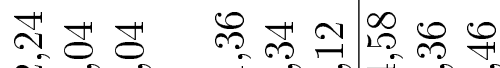

Iิ

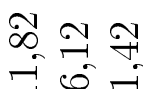

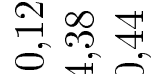

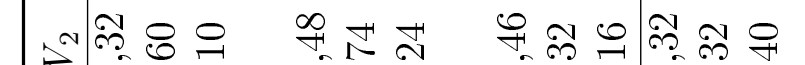

유 $\exists$ छे $\exists 10^{\circ}-100$

$\infty$ i i ปี่

\&

3 뉴

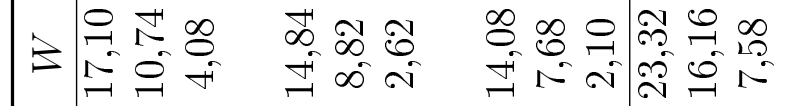

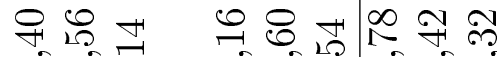

อิ

I 0 ต

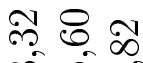

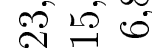

บิ

श

년

군

0 is

int 0

$\infty \stackrel{\infty}{\mathbb{1}}$

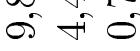

$\infty \infty$

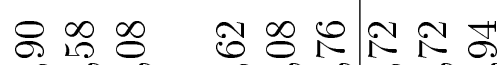

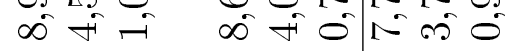

$\infty$

于1

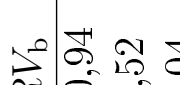

के

क十

$\infty \infty$ \&

in

+

$\infty 8$

$\infty \stackrel{\circ}{2} \sim$

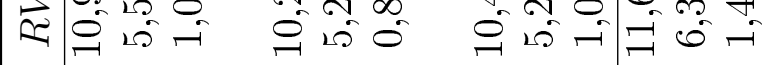

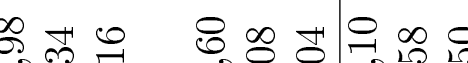

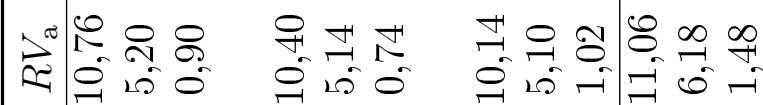

0 is

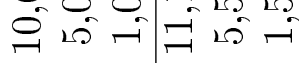

مิ

$\vec{N} \exists 10$

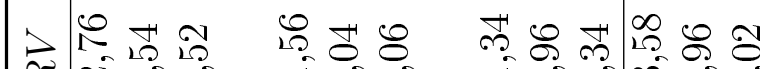

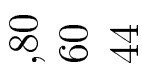

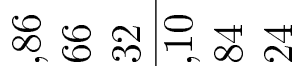

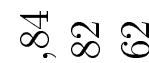

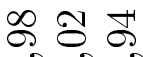
2100

$\frac{\pi}{8}$

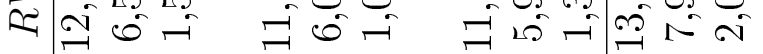

ำ

$\exists$ is $-100 \mathrm{~N}$

I 0

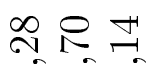

of

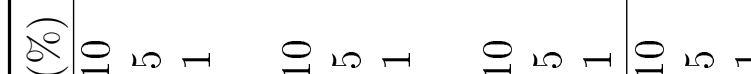

$\bigcirc 10-$

$ㅇ ㅛ-1010-$

$\bigoplus 10-$

$\exists 88$ $\exists=15$ | คి

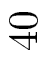

จ

$\stackrel{8}{\infty}$

$\vartheta$

๙

คి 
Tabela 3.12: Poder para $\mathcal{H}_{1}: \lambda_{2}=\epsilon ; n=30, p=1, q=2, \alpha=10 \%$ e distribuição normal inversa.

\begin{tabular}{c|ccccccccc}
\hline$\epsilon$ & $R V_{\mathrm{a}}$ & $R V_{\mathrm{b}}$ & $S R_{\mathrm{b}}$ & $W_{2}$ & $T_{\mathrm{b}}$ & $R V_{\text {boot }}$ & $S R_{\text {boot }}$ & $W_{\text {boot }}$ & $T_{\text {boot }}$ \\
\hline 0,5 & 14,36 & 14,14 & 13,72 & 13,64 & 14,06 & 14,20 & 14,36 & 14,20 & 14,12 \\
1,5 & 43,82 & 42,86 & 41,20 & 40,70 & 42,78 & 42,86 & 41,60 & 40,78 & 42,94 \\
2,5 & 77,40 & 76,74 & 72,94 & 75,78 & 76,58 & 76,42 & 73,22 & 75,76 & 76,38 \\
3,5 & 93,94 & 93,78 & 90,56 & 94,28 & 93,66 & 93,64 & 90,82 & 94,28 & 93,64 \\
\hline
\end{tabular}

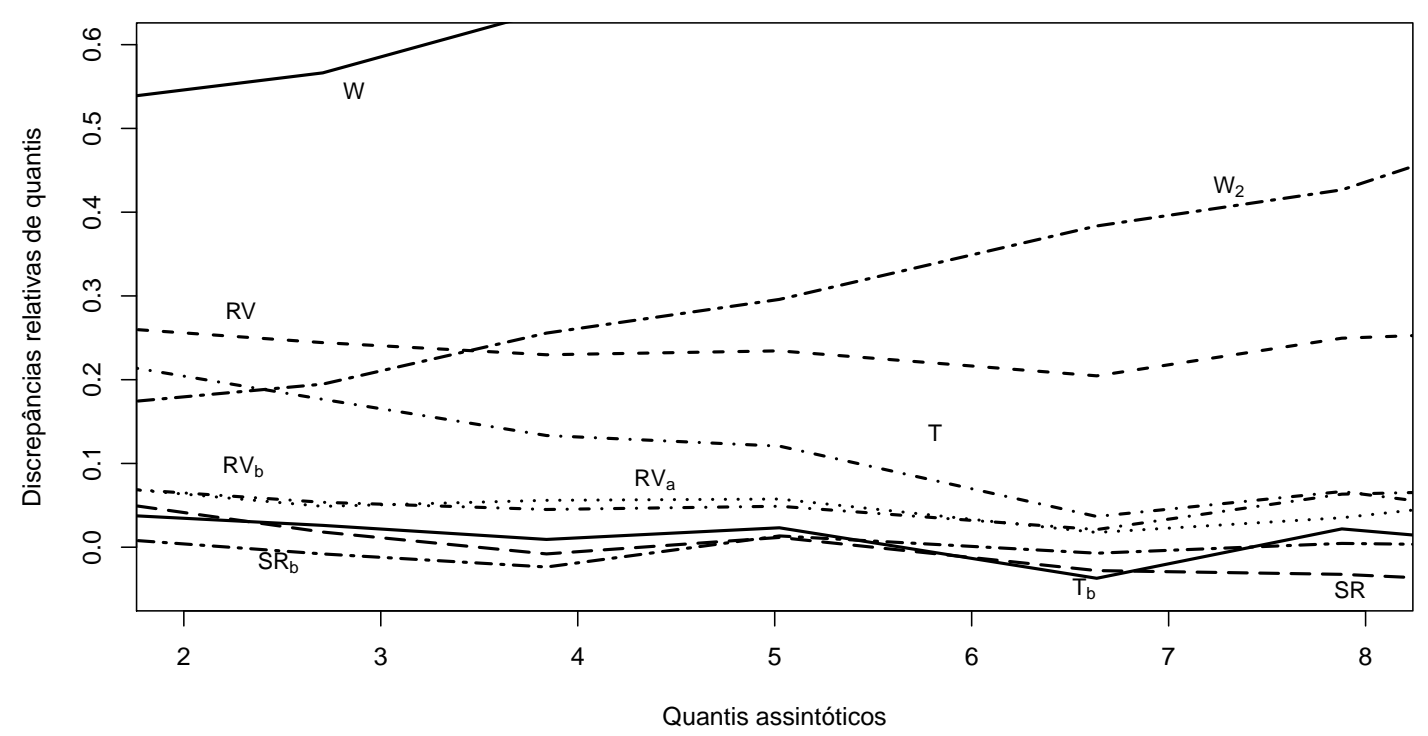

Figura 3.2: Discrepâncias relativas de quantis - modelo normal inversa com $n=40, p=3$ e $q=3$.

\subsection{Aplicação}

Para ilustrar uma aplicação das estatísticas da razão de verossimilhanças, escore, Wald e gradiente usuais, suas versões corrigidas e Wald modificada, consideramos o conjunto de dados analisados em Simonoff e Tsai (1994). Estes dados representam as taxas mensais de retorno de ações de mercado $(x)$ e da Corporação Acme Cleveland $(Y)$, no período de Janeiro de 1986 a Dezembro de 1990. Na análise original destes dados, os autores sugeriram um modelo de regressão normal heteroscedástico $N\left(\mu_{\ell}, \phi_{\ell}^{-1}\right)$, para prever $Y_{\ell}$ em termos de uma função linear do retorno do mercado $x_{\ell}$, considerando os componentes sistemáticos para a média e para a precisão: $\mu_{\ell}=\beta_{1}+\beta_{2} x_{\ell} \mathrm{e}-\log \left(\phi_{\ell}\right)=\lambda_{1}+\lambda_{2} x_{\ell}$, para $\ell=1, \ldots, 59 \mathrm{em}$ que a observação 22 , a qual reflete que o mercado perdeu aproximadamente um terço de seu valor durante um período de duas semanas no mês de Outubro de 1987 (ver Simonoff e Tsai (1994, p. 359)), foi removida a partir da análise. 
Estamos interessados em testar a hipótese nula $\mathcal{H}_{0}: \lambda_{2}=0$ (homocedasticidade).

As estimativas dos parâmetros encontram-se na Tabela 3.13. Podemos observar na Tabela 3.14 que se considerarmos o nível nominal de 5\% a hipótese nula somente é rejeitada quando a inferência é baseada no teste Wald usual. No entanto, se o nível nominal considerado for de $10 \%$, a hipótese nula só não é rejeitada pelo teste escore.

Tabela 3.13: Estimativas dos parâmetros.

\begin{tabular}{lcccc}
\hline Parâmetro & $\beta_{1}$ & $\beta_{2}$ & $\lambda_{1}$ & $\lambda_{2}$ \\
\hline Estimativa & $-0,0049$ & 1,2528 & $-4,4104$ & 8,0923 \\
Erro padrão & 0,0197 & 0,2487 & 0,2651 & 4,0088 \\
\hline
\end{tabular}

Tabela 3.14: Valores das estatísticas de teste e p-valores para testar $\mathcal{H}_{0}: \lambda_{2}=0$.

\begin{tabular}{lcc}
\hline Estatística & valor & p-valor \\
\hline$R V$ & 3,3286 & 0,0681 \\
$R V_{\mathrm{a}}$ & 3,0950 & 0,0785 \\
$R V_{\mathrm{b}}$ & 3,1204 & 0,0773 \\
$S R$ & 2,6984 & 0,1004 \\
$S R_{\mathrm{b}}$ & 2,8886 & 0,0892 \\
$W$ & 4,0748 & 0,0435 \\
$W_{2}$ & 3,3916 & 0,0655 \\
$T$ & 3,3160 & 0,0686 \\
$T_{\mathrm{b}}$ & 3,0860 & 0,0790 \\
$R V_{\text {boot }}$ & & 0,0760 \\
$S R_{\text {boot }}$ & & 0,0805 \\
$W_{\text {boot }}$ & & 0,0775 \\
$T_{\text {boot }}$ & & 0,0765 \\
\hline
\end{tabular}

\subsection{Conclusões}

Em linhas gerais, os testes da razão de verossimilhanças, Wald, Wald modificado e gradiente podem ser demasiadamente liberais em amostras de tamanho pequeno, rejeitando a hipótese nula muito mais frequentemente do que o esperado com base no nível nominal selecionado. O teste escore mostrou-se menos distorcido, podendo ser conservativo em alguns casos. O fator de correção tipo-Bartlett para os testes escore e gradiente faz com que a taxa de rejeição empírica fique mais próxima do nível nominal do teste, sendo este fato mais perceptível no que se refere ao teste gradiente usual. Os testes da razão de verossimilhanças ajustado e corrigido apresentam maior distorção de tamanho que os testes escore e gradiente corrigidos. Os testes bootstrap, apesar de computacionalmente custosos, 
conduziram a uma considerável redução da distorção de tamanho observada para os testes assintóticos, especialmente para os testes da razão de verossimilhanças e Wald, cujas versões usuais são tipicamente liberais. Ainda que os testes escore e gradiente corrigidos e os testes bootstrap exijam algum esforço computacional, eles são menos distorcidos, na maioria dos casos analisados, sendo portanto, recomendados em aplicações práticas. 


\section{Capítulo 4}

\section{Coeficiente de Assimetria}

Neste capítulo, desenvolvemos expressões matriciais de ordem $n^{-1 / 2}$ para o coeficiente de assimetria da distribuição do EMV em MLGHs. Estas expressões podem ser utilizadas para averiguar se a distribuição do EMV encontra-se próxima da distribuição normal. Estudos de simulação foram realizados a fim de avaliar a assimetria da distribuição do EMV.

\subsection{Introdução}

Uma possível medida utilizada para verificar a não normalidade de uma distribuição é a assimetria. O coeficiente de assimetria é comumente definido como o terceiro cumulante padronizado de Pearson, dado por $\gamma_{1}=\kappa_{3} / \kappa_{2}^{3 / 2}$, em que $\kappa_{r}$ é o $r$-ésimo cumulante da distribuição. Distribuições para as quais $\gamma_{1}>0\left(\gamma_{1}<0\right)$ são positivamente (negativamente) assimétricas e terão uma cauda mais longa (curta) à direita e mais curta (longa) à esquerda. Distribuições simétricas possuem $\gamma_{1}=0$. No entanto, $\gamma_{1}=0$ não implica em simetria. Um exemplo disto é apresentado em Kendall e Stuart (1977, Exercício 3.26), em que a distribuição é assimétrica e possui os cumulantes de ordem ímpar iguais a zero.

Bowman e Shenton (1998) expandiram o terceiro momento central da distribuição dos EMVs até a ordem $n^{-2}$ e, a partir desta expansão, obtiveram o coeficiente de assimetria até a ordem $n^{-1 / 2}$ da distribuição dos EMVs.

O objetivo deste capítulo é obter o coeficiente de assimetria de ordem $n^{-1 / 2}$ da distribuição dos EMVs em MLGHs, estendendo assim os resultados de Cordeiro e Cordeiro (2001).

\subsection{Coeficiente de assimetria da distribuição do EMV}

Consideremos um modelo de regressão qualquer, indexado pelo vetor de parâmetros $\boldsymbol{\theta}=$ $\left(\theta_{1}, \ldots, \theta_{k}\right)^{\top}$. Seja $\kappa_{3}\left(\hat{\theta}_{a}\right)=E\left[\hat{\theta}_{a}-E\left(\hat{\theta}_{a}\right)\right]^{3}$ o terceiro momento central do EMV de $\theta_{a}, \hat{\theta}_{a}$, para $a=1, \ldots, k$. A expressão geral para o terceiro momento central de ordem $n^{-2}$ dos 
EMVs é dada em Bowman e Shenton (1998) por

$$
\kappa_{3}\left(\hat{\theta}_{a}\right)=\sum \kappa^{a, r} \kappa^{a, s} \kappa^{a, t}\left(\kappa_{r, s, t}+3 \kappa_{r s t}+6 \kappa_{r s, t}\right)
$$

em que o somatório é sobre todos os $k$ parâmetros $\theta_{1}, \ldots, \theta_{k}$.

\subsubsection{Coeficiente de assimetria para a distribuição de $\hat{\beta}$}

Seja $\boldsymbol{\theta}=\left(\boldsymbol{\beta}^{\top}, \boldsymbol{\lambda}^{\top}\right)^{\top}$ o vetor de parâmetros de dimensão $p+q \operatorname{com} \boldsymbol{\beta}=\left(\beta_{1}, \ldots, \beta_{p}\right)^{\top}$ e $\boldsymbol{\lambda}=\left(\lambda_{1}, \ldots, \lambda_{q}\right)^{\top}$ associado ao MLGH definido por (1.1) e (1.2). Da expressão (4.1) podemos escrever o terceiro momento central do EMV de $\beta_{a}, \hat{\beta}_{a}$, para $a=1, \ldots, p$ como

$$
\kappa_{3}\left(\hat{\beta}_{a}\right)=\sum_{\beta, \lambda} \kappa^{a, r} \kappa^{a, s} \kappa^{a, t}\left(\kappa_{r, s, t}+3 \kappa_{r s t}+6 \kappa_{r s, t}\right)
$$

em que o somatório é sobre todas as combinações dos $(p+q)$ parâmetros $\beta_{1}, \ldots, \beta_{p}$ e $\lambda_{1}, \ldots, \lambda_{q}$. A expressão (4.2) é simples de calcular devido à invariância dos $\kappa$ 's sob permutação dos parâmetros $\boldsymbol{\beta}$ e devido à ortogonalidade entre $\boldsymbol{\beta}$ e $\boldsymbol{\lambda}$.

Utilizando os cumulantes definidos no Apêndice A, obtemos

$$
\begin{aligned}
\kappa_{r, s, t}+3 \kappa_{r s t}+6 \kappa_{r s, t} & =\sum_{\ell=1}^{n} \phi_{\ell}\left[\left(f_{\ell}-g_{\ell}\right)-3\left(f_{\ell}+2 g_{\ell}\right)+6 g_{\ell}\right] x_{\ell r} x_{\ell s} x_{\ell t} \\
& =-\sum_{\ell=1}^{n} \phi_{\ell}\left(2 f_{\ell}+g_{\ell}\right) x_{\ell r} x_{\ell s} x_{\ell t} .
\end{aligned}
$$

Substituindo (4.3) em (4.2) e invertendo a ordem dos somatórios, obtemos

$$
\kappa_{3}\left(\hat{\beta}_{a}\right)=-\sum_{\ell=1}^{n}\left\{\left(\sum_{r=1}^{p} \kappa^{a, r} x_{r \ell}\right)\left(\sum_{s=1}^{p} \kappa^{a, s} x_{s \ell}\right)\left(\sum_{t=1}^{p} \kappa^{a, t} x_{t \ell}\right)\left[\phi_{\ell}\left(2 f_{\ell}+g_{\ell}\right)\right]\right\} .
$$

Segue portanto que a expressão (4.4) pode ser escrita em notação matricial como

$$
\kappa_{3}(\hat{\boldsymbol{\beta}})=-\left(\boldsymbol{P}_{\beta} \odot \boldsymbol{P}_{\beta} \odot \boldsymbol{P}_{\beta}\right) \boldsymbol{\Phi}(2 \boldsymbol{F}+\boldsymbol{G}) \mathbf{1},
$$

em que $\boldsymbol{P}_{\beta}=\left(\boldsymbol{X}^{\top} \boldsymbol{W} \boldsymbol{\Phi} \boldsymbol{X}\right)^{-1} \boldsymbol{X}^{\top}, \boldsymbol{F}=\operatorname{diag}\left\{f_{1}, \ldots, f_{n}\right\} \operatorname{com} f_{\ell}=V_{\ell}^{-1}\left(\mathrm{~d} \mu_{\ell} / \mathrm{d} \eta_{\ell}\right)\left(\mathrm{d}^{2} \mu_{\ell} / \mathrm{d} \eta_{\ell}^{2}\right)$, $\boldsymbol{G}=\operatorname{diag}\left\{g_{1}, \ldots, g_{n}\right\} \operatorname{com} g_{\ell}=f_{\ell}-V_{\ell}^{-2} \mathrm{~d} V_{\ell} / \mathrm{d} \mu_{\ell}\left(\mathrm{d} \mu_{\ell} / \mathrm{d} \eta_{\ell}\right)^{3}, \boldsymbol{\Phi}=\operatorname{diag}\left\{\phi_{1}, \ldots, \phi_{n}\right\}, \mathbf{1}$ é um vetor de uns de dimensão $n \times 1$ e $\odot$ denota o produto direto entre matrizes.

A expressão (4.5) generaliza a expressão (2.5) em Cordeiro e Cordeiro (2001). 


\subsubsection{Coeficiente de assimetria para a distribuição de $\hat{\lambda}$}

Utilizando o mesmo contexto da Subseção 4.2.1 podemos escrever o terceiro momento central do EMV de $\lambda_{A}, \hat{\lambda}_{A}$, para $A=1, \ldots, q$ como

$$
\kappa_{3}\left(\hat{\lambda}_{A}\right)=\sum_{\beta, \lambda} \kappa^{A, R} \kappa^{A, S} \kappa^{A, T}\left(\kappa_{R, S, T}+3 \kappa_{R S T}+6 \kappa_{R S, T}\right)
$$

em que o somatório é sobre todas as combinações dos $(p+q)$ parâmetros $\beta_{1}, \ldots, \beta_{p}$ e $\lambda_{1}, \ldots, \lambda_{q}$. A expressão (4.6) é simples de calcular devido à invariância dos $\kappa$ 's sob permutação dos parâmetros $\boldsymbol{\lambda}$ e devido à ortogonalidade entre $\boldsymbol{\beta}$ e $\boldsymbol{\lambda}$.

Utilizando os cumulantes definidos no Apêndice A, obtemos

$$
\begin{aligned}
\kappa_{R, S, T}+3 \kappa_{R S T}+6 \kappa_{R S, T} & =\sum_{\ell=1}^{n}\left[-d_{3 \ell} \phi_{1 \ell}^{3}+3\left(d_{3 \ell} \phi_{1 \ell}^{3}+2 d_{2 \ell} \phi_{1 \ell} \phi_{2 \ell}\right)-6 d_{2 \ell} \phi_{1 \ell} \phi_{2 \ell}\right] s_{\ell R} s_{\ell S} x_{\ell T} \\
& =\sum_{\ell=1}^{n}\left(2 d_{3 \ell} \phi_{1 \ell}^{3}+3 d_{2 \ell} \phi_{1 \ell} \phi_{2 \ell}\right) s_{\ell R} s_{\ell S} x_{\ell T}
\end{aligned}
$$

Substituindo (4.7) em (4.6) e invertendo a ordem dos somatórios, obtemos

$$
\kappa_{3}\left(\hat{\lambda}_{A}\right)=\sum_{\ell=1}^{n}\left\{\left(\sum_{R=1}^{q} \kappa^{A, R} s_{R \ell}\right)\left(\sum_{S=1}^{q} \kappa^{A, S} s_{S \ell}\right)\left(\sum_{T=1}^{q} \kappa^{A, T} s_{T \ell}\right)\left(2 d_{3 \ell} \phi_{1 \ell}^{3}+3 d_{2 \ell} \phi_{1 \ell} \phi_{2 \ell}\right)\right\} .
$$

Segue portanto que a expressão (4.8) pode ser escrita em notação matricial como

$$
\kappa_{3}(\hat{\boldsymbol{\lambda}})=\left(\boldsymbol{P}_{\lambda} \odot \boldsymbol{P}_{\lambda} \odot \boldsymbol{P}_{\lambda}\right)\left(2 \boldsymbol{D}_{3} \boldsymbol{\Phi}_{1}^{3}+3 \boldsymbol{D}_{2} \boldsymbol{\Phi}_{1} \boldsymbol{\Phi}_{2}\right) \mathbf{1}
$$

em que $\boldsymbol{P}_{\lambda}=\left(-\boldsymbol{S}^{\top} \boldsymbol{D}_{2} \boldsymbol{\Phi}_{1}^{2} \boldsymbol{S}\right)^{-1} \boldsymbol{S}^{\top}, \boldsymbol{\Phi}_{1}=\operatorname{diag}\left\{\phi_{11}, \ldots, \phi_{1 n}\right\}, \boldsymbol{\Phi}_{2}=\operatorname{diag}\left\{\phi_{21}, \ldots, \phi_{2 n}\right\}, \boldsymbol{D}_{2}=$ $\operatorname{diag}\left\{d_{21}, \ldots, d_{2 n}\right\}, \boldsymbol{D}_{3}=\operatorname{diag}\left\{d_{31}, \ldots, d_{3 n}\right\}$ sendo $\phi_{k \ell}=\mathrm{d}^{k} \phi_{\ell} / \mathrm{d} \tau_{\ell}^{k}$ e $d_{k \ell}=\mathrm{d}^{k} d\left(\phi_{\ell}\right) / \mathrm{d} \phi_{\ell}^{k}$, para $k=1, \ldots, 4$ e $\ell=1, \ldots, n, 1$ é um vetor de uns de dimensão $n \times 1$ e $\odot$ denota o produto direto entre matrizes.

A expressão (4.9) generaliza a expressão (4.1) em Cordeiro e Cordeiro (2001).

\subsection{Resultados numéricos}

Apresentamos a seguir alguns resultados de simulações de Monte Carlo para comparar o coeficiente de assimetria de ordem $n^{-1 / 2}$ com o coeficiente de assimetria amostral, o qual é definido pela razão dos momentos amostrais. As simulações realizadas foram baseadas nos 
modelos de regressão gama e normal inversa com componentes sistemáticas definidas por

$$
\mu_{\ell}=\beta_{1}+\beta_{2} x_{\ell 2} \quad \text { e } \quad \log \left(\phi_{\ell}\right)=\lambda_{1}+\lambda_{2} s_{\ell 2}
$$

em que a covariável $x_{2}$ foi obtida a partir das distribuições $\mathcal{U}(0,1)$ e Exponencial(6) e consideramos $s_{2}=x_{2}$. Para cada tamanho da amostra as covariáveis foram mantidas constantes durante as simulações. Os valores verdadeiros dos parâmetros foram fixados em $\beta_{1}=2$, $\beta_{2}=3, \lambda_{1}=4$ e $\lambda_{2}=2$.

A quantidade de réplicas foi estabelecida em 10000. Em cada réplica, ajustamos o modelo e calculamos o seu EMV $\hat{\boldsymbol{\theta}}$. O coeficiente de assimetria amostral é definido por $\boldsymbol{g}_{1}=m_{3} / \mathrm{m}_{2}^{3 / 2}$, em que $m_{r}=\sum_{i=1}^{10000}\left(\hat{\boldsymbol{\theta}}_{i}-\overline{\hat{\boldsymbol{\theta}}}\right)^{r} / 10000$ para $r=2$ e 3 e $\overline{\hat{\boldsymbol{\theta}}}=\sum_{i=1}^{10000} \hat{\boldsymbol{\theta}}_{i} / 10000$. Todas as simulações foram realizadas utilizando a linguagem de programação matricial ox (Doornik, 2006).

Podemos fazer alguns comentários referentes às Tabelas 4.1 e 4.2. Primeiro, a diferença entre os valores dos coeficientes de assimetria de ordem $n^{-1 / 2}$ e amostral é maior quando o tamanho da amostra é pequeno. Segundo, a distribuição da variável resposta possui uma maior influencia sobre os coeficientes de assimetria assintótico e amostral nas distribuições dos EMVs dos parâmetros $\beta_{1}$ e $\beta_{2}$. Terceiro, os coeficientes de assimetria assintóticos das distribuições dos EMVs dos parâmetros da dispersão são praticamente os mesmos independentemente da distribuição da variável resposta. Quarto, a mudança da distribuição das covariáveis produziu resultados diferentes para os coeficientes de assimetria amostral e assintótico, uma vez que, para os modelos com covariável Exponencial(6), os coeficientes de assimetria são todos positivos, enquanto que, para os modelos com covariável $\mathcal{U}(0,1)$, os coeficientes de assimetria para as distribuições dos parâmetros $\hat{\boldsymbol{\beta}}_{2}$ e $\hat{\boldsymbol{\lambda}}_{2}$ são negativos.

Nas Figuras 4.1 a 4.4 comparamos as densidades estimadas dos EMVs com a densidade da distribuição normal. Os parâmetros utilizados para a distribuição normal são as médias e as variâncias obtidas dos respectivos EMVs. Podemos perceber nas Figuras 4.1 e 4.2 uma leve assimetria da distribuição de $\hat{\lambda}_{2}$ e uma assimetria um pouco mais acentuada para a distribuição de $\hat{\lambda}_{1}$. Já nas Figuras 4.3 e 4.4 percebemos uma assimetria mais acentuada para a distribuição de $\hat{\lambda}_{2}$. De um modo geral, nas Figuras 4.1 a 4.4 , as distribuições de $\hat{\beta}_{1}$ e $\hat{\beta}_{2}$ são praticamente simétricas.

\subsection{Conclusões}

Neste capítulo, consideramos a classe dos MLGHs e obtivemos fórmulas matriciais para o coeficiente de assimetria de ordem $n^{-1 / 2}$. Estas fórmulas são úteis para verificar se a aproximação da distribuição do EMV em relação à distribuição normal é adequada em amostras de tamanho pequeno a moderado. Os estudos de simulação indicaram que, em geral, o coeficiente de assimetria da distribuição dos EMVs é influenciado tanto pela distribuição da 
variável resposta quanto pela distribuição da covariável. Estes estudos também mostraram, de modo geral, que a distribuição de $\hat{\boldsymbol{\beta}}$ é mais simétrica que a de $\hat{\boldsymbol{\lambda}}$.

Tabela 4.1: Coeficiente de assimetria amostral e de ordem $n^{-1 / 2}$ e covariável $\mathcal{U}(0,1)$.

\begin{tabular}{|c|c|c|c|c|c|c|c|c|c|}
\hline & \multirow[b]{2}{*}{$n$} & \multicolumn{4}{|c|}{ modelo gama } & \multicolumn{4}{|c|}{ modelo normal inversa } \\
\hline & & $\hat{\beta}_{1}$ & $\hat{\beta}_{2}$ & $\hat{\lambda}_{1}$ & $\hat{\lambda}_{2}$ & $\hat{\beta}_{1}$ & $\hat{\beta}_{2}$ & $\hat{\lambda}_{1}$ & $\hat{\lambda}_{2}$ \\
\hline$g_{1}$ & 20 & 0,0996 & $-0,0136$ & 0,4963 & $-0,1222$ & 0,4294 & $-0,1256$ & 0,5226 & $-0,1510$ \\
\hline$\gamma_{1}$ & 20 & 0,0671 & $-0,0226$ & 0,4312 & $-0,0705$ & 0,1674 & $-0,0309$ & 0,4277 & $-0,0691$ \\
\hline$g_{1}$ & 40 & 0,0743 & $-0,0378$ & 0,2759 & $-0,0178$ & 0,1909 & $-0,0504$ & 0,2532 & $-0,0238$ \\
\hline$\gamma_{1}$ & 40 & 0,0455 & $-0,0165$ & 0,2741 & $-0,0181$ & 0,1231 & $-0,0295$ & 0,2717 & $-0,0170$ \\
\hline$g_{1}$ & 60 & 0,0452 & $-0,0143$ & 0,2402 & $-0,0119$ & 0,1350 & $-0,0299$ & 0,1858 & $-0,0046$ \\
\hline$\gamma_{1}$ & 00 & 0,0380 & $-0,0139$ & 0,2247 & $-0,0196$ & 0,1028 & $-0,0256$ & 0,2227 & $-0,0187$ \\
\hline$g_{1}$ & 80 & 0,0374 & $-0,0043$ & 0,1891 & $-0,0126$ & 0,0879 & $-0,0013$ & 0,2018 & $-0,0221$ \\
\hline$\gamma_{1}$ & & 0,0333 & $-0,0106$ & 0,1897 & $-0,0010$ & 0,0891 & $-0,0176$ & 0,1880 & $-0,0002$ \\
\hline
\end{tabular}

Tabela 4.2: Coeficiente de assimetria amostral e de ordem $n^{-1 / 2}$ e covariável Exponencial(6).

\begin{tabular}{|c|c|c|c|c|c|c|c|c|c|}
\hline & \multirow[b]{2}{*}{$n$} & \multicolumn{4}{|c|}{ modelo gama } & \multicolumn{4}{|c|}{ modelo normal inversa } \\
\hline & & $\hat{\beta}_{1}$ & $\hat{\beta}_{2}$ & $\hat{\lambda}_{1}$ & $\hat{\lambda}_{2}$ & $\hat{\beta}_{1}$ & $\hat{\beta}_{2}$ & $\hat{\lambda}_{1}$ & $\hat{\lambda}_{2}$ \\
\hline$g_{1}$ & & 0,1458 & 0,0839 & 0,0741 & 0,6742 & 0,2203 & 0,4446 & 0,0640 & 0,7078 \\
\hline$\gamma_{1}$ & 20 & 0,0448 & 0,0251 & 0,2450 & 0,3991 & 0,0977 & 0,0814 & 0,2426 & 0,3981 \\
\hline$g_{1}$ & & 0,0400 & 0,0022 & 0,1535 & 0,4015 & 0,1026 & 0,1810 & 0,0947 & 0,4013 \\
\hline$\gamma_{1}$ & 40 & 0,0358 & 0,0287 & 0,1805 & 0,3394 & 0,0797 & 0,0812 & 0,1787 & 0,3387 \\
\hline$g_{1}$ & 60 & 0,0101 & 0,0239 & 0,0811 & 0,3641 & 0,0637 & 0,1342 & 0,1094 & 0,2920 \\
\hline$\gamma_{1}$ & 00 & 0,0282 & 0,0263 & 0,1390 & 0,2779 & 0,0620 & 0,0724 & 0,1376 & 0,2773 \\
\hline$g_{1}$ & 80 & 0,0075 & 0,0135 & 0,0849 & 0,3293 & 0,0412 & 0,0700 & 0,0710 & 0,3698 \\
\hline$\gamma_{1}$ & 80 & 0,0222 & 0,0266 & 0,0965 & 0,3128 & 0,0491 & 0,0726 & 0,0953 & 0,3125 \\
\hline
\end{tabular}



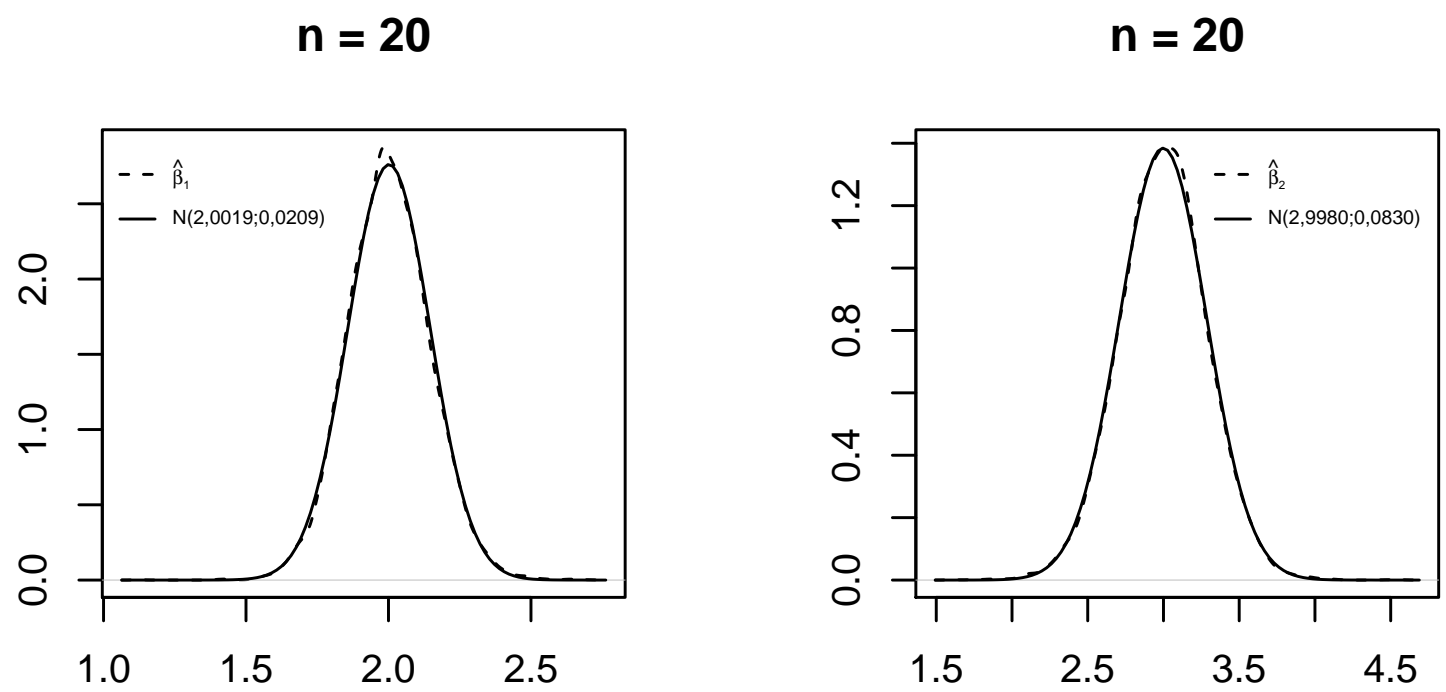

$\mathbf{n}=\mathbf{2 0}$

$n=20$
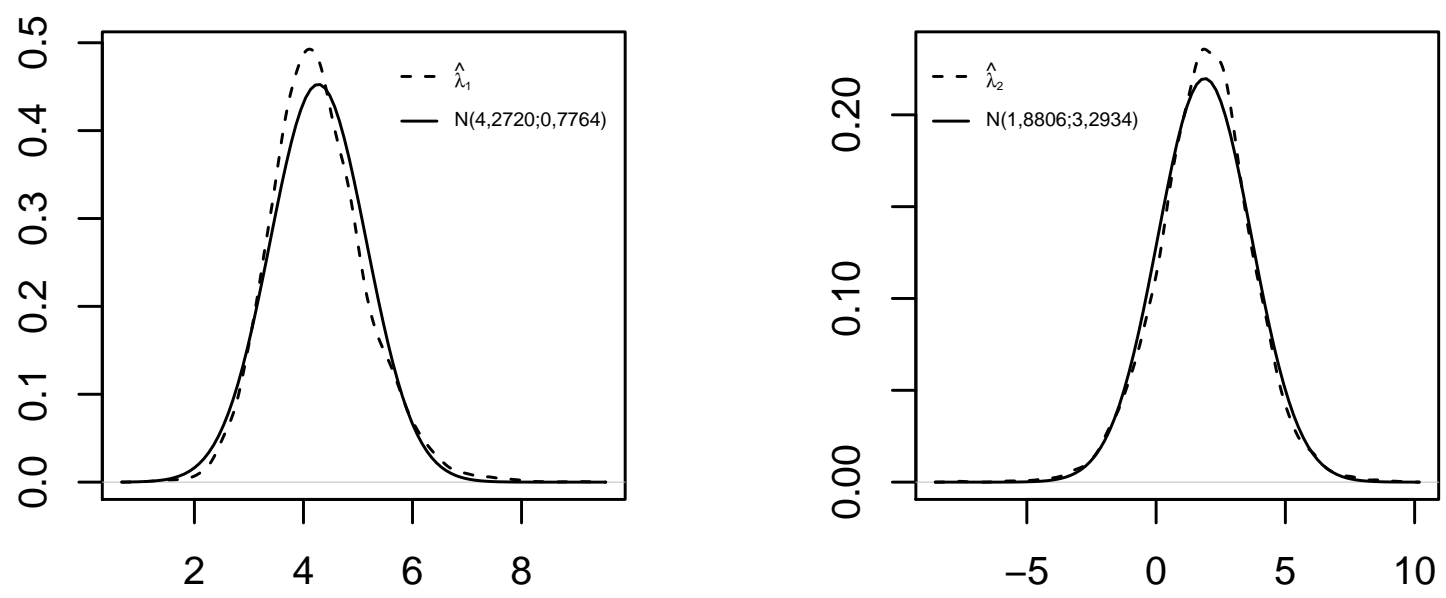

Figura 4.1: Densidades estimada e normal; modelo gama e covariável $\mathcal{U}(0,1)$. 

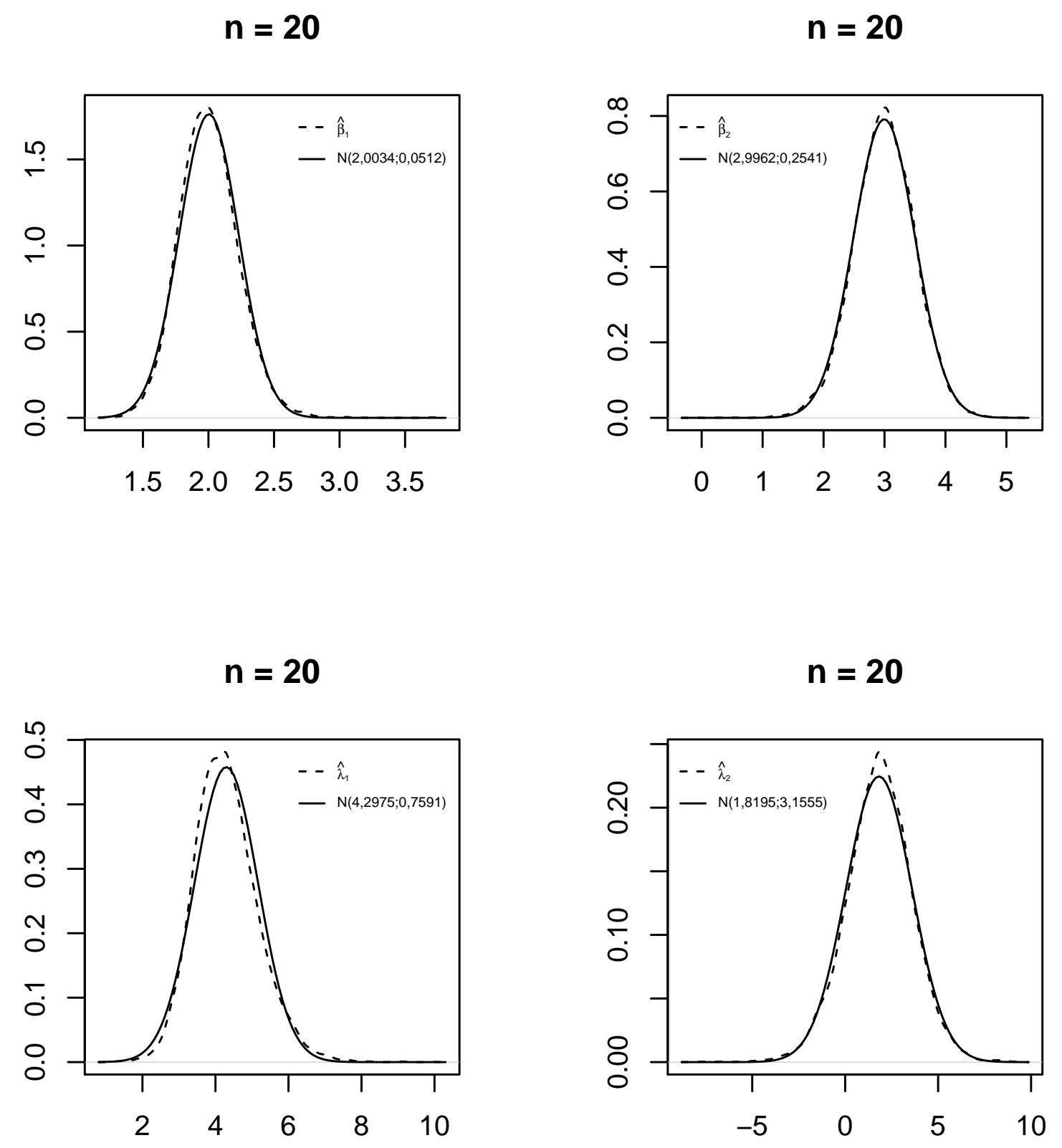

Figura 4.2: Densidades estimada e normal; modelo normal inversa e covariável $\mathcal{U}(0,1)$. 

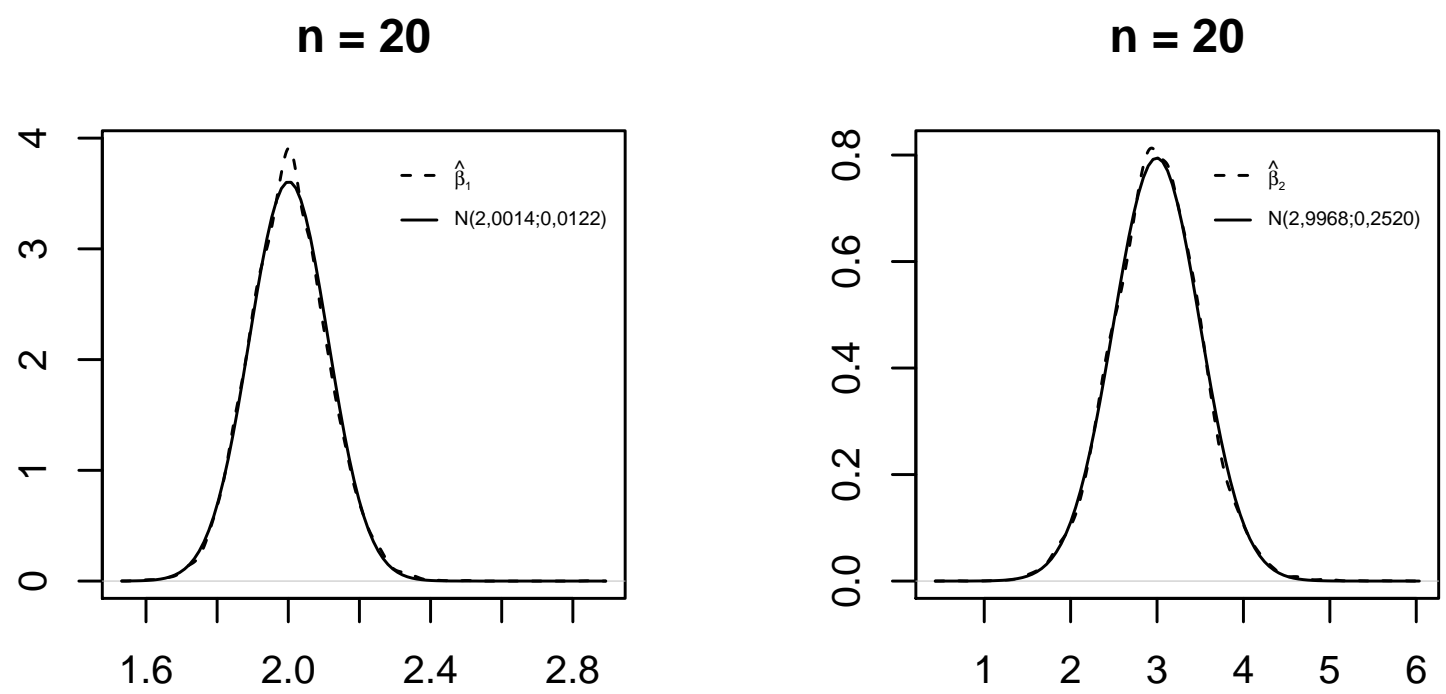

$$
\mathbf{n}=\mathbf{2 0}
$$
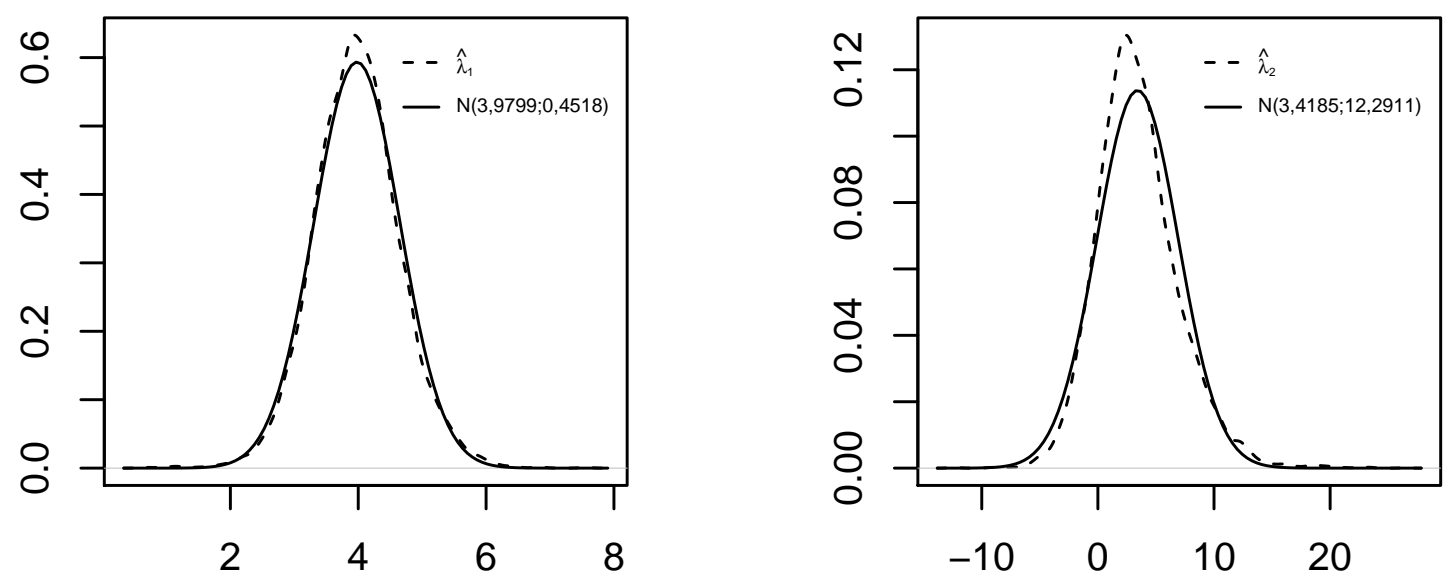

Figura 4.3: Densidades estimada e normal; modelo gama e covariável Exponencial(6). 

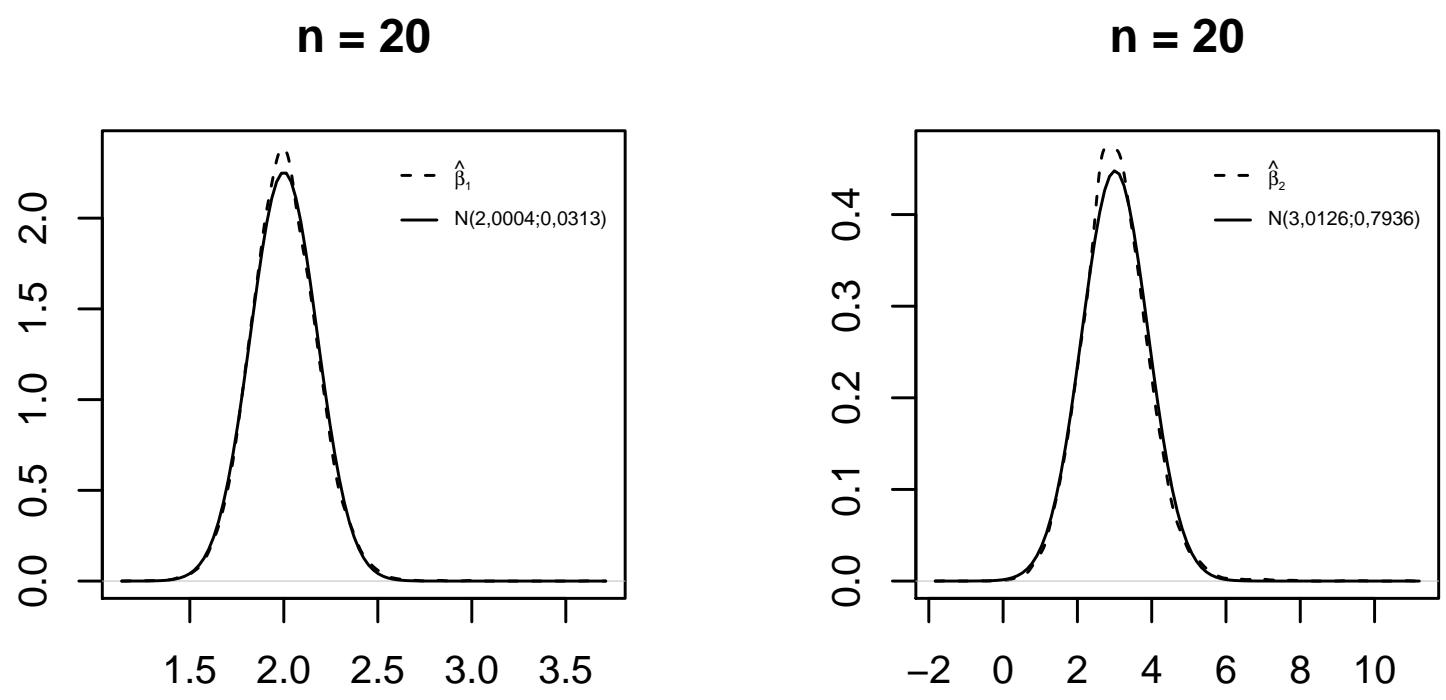

$\mathbf{n}=\mathbf{2 0}$

$\mathrm{n}=\mathbf{2 0}$
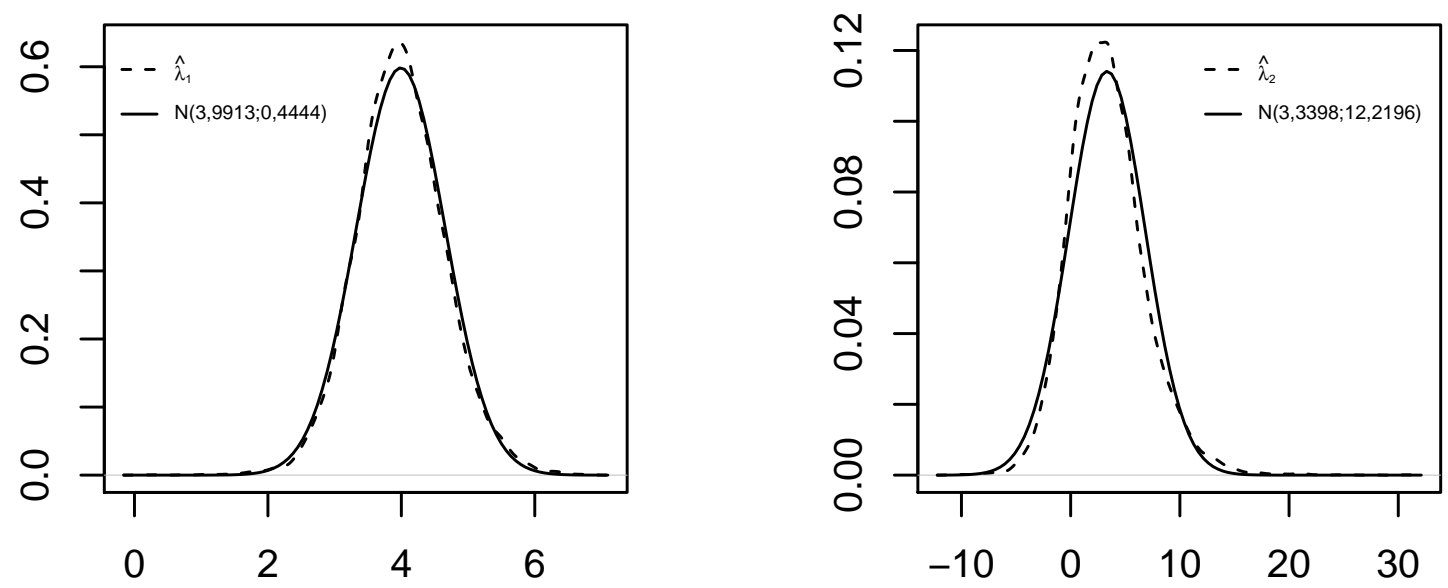

Figura 4.4: Densidades estimada e normal; modelo normal inversa e covariável Exponencial(6). 


\section{Capítulo 5}

\section{Coeficiente de Curtose}

Neste capítulo, desenvolvemos expressões matriciais de ordem $n^{-1}$ para o coeficiente de curtose da distribuição do EMV em MLGHs. A ideia por trás desta expressão é verificar se a aproximação da distribuição do EMV pela distribuição normal é apropriada quando o tamanho da amostra é pequeno ou moderado. Estudos de simulação foram realizados com o intuito de avaliar a curtose da distribuição do EMV. Por fim, aplicamos os resultados obtidos a um conjunto de dados reais.

\subsection{Introdução}

Curtose é uma medida amplamente utilizada para caracterizar o grau de achatamento da distribuição, frequentemente estabelecida em relação à distribuição normal. O coeficiente de curtose, também denominado de excesso de curtose, é definido como o quarto cumulante padronizado de Pearson, a saber, $\gamma=\kappa_{4} / \kappa_{2}^{2}=\left(\mu_{4} / \mu_{2}^{2}\right)-3$, em que $\kappa_{r}$ é o $r$-ésimo cumulante da distribuição e $\mu_{r}$ é o $r$-ésimo momento central da distribuição.

Distribuições para as quais $\gamma=0$ são chamadas mesocúrticas e possuem o mesmo achatamento da distribuição normal. As distribuições para as quais $\gamma>0$ são chamadas leptocúrticas e aquelas para as quais $\gamma<0$ são chamadas platicúrticas. A distribuição leptocúrtica tem um pico mais acentuado e caudas mais longas e mais pesadas, enquanto que a distribuição platicúrtica é caracterizada por um pico mais arredondado e caudas mais curtas e mais magras. O coeficiente de curtose está definido em $[-2,+\infty)$.

Bowman e Shenton (1999) expandiram o quarto momento central da distribuição dos EMVs até a ordem $n^{-3}$ e apresentaram uma nova definição para o coeficiente de curtose até a ordem $n^{-1}$ da distribuição dos EMVs.

O objetivo deste capítulo é obter o coeficiente de curtose até ordem $n^{-1}$ da distribuição dos EMVs em MLGHs. 


\subsection{Coeficiente de curtose da distribuição do EMV}

Consideremos um modelo de regressão qualquer, indexado pelo vetor de parâmetros $\boldsymbol{\theta}=$ $\left(\theta_{1}, \ldots, \theta_{k}\right)^{\top}$. Seja $\mu_{4}\left(\hat{\theta}_{a}\right)$ o quarto momento central de ordem $n^{-3}$ do EMV de $\theta_{a}, \hat{\theta}_{a}$, para $a=1, \ldots, k$. A sua expressão geral é dada em Bowman e Shenton (1998) por

$$
\mu_{4}\left(\hat{\theta}_{a}\right)=A_{40}+2 A_{32}+\frac{2}{3} A_{33}+\frac{3}{2} A_{22}
$$

em que

$$
\begin{gathered}
A_{40}=\sum \kappa^{a, r} \kappa^{a, s} \kappa^{a, t} \kappa^{a, u} \kappa_{r, s, t, u} \\
A_{32}=2 \sum \kappa^{a, r} \kappa^{s, t} \kappa^{a, u} \kappa^{a, v} \kappa^{a, w}\left(\kappa_{r s, t} \kappa_{u, v, w}+\kappa_{r s, u} \kappa_{t, v, w}+\kappa_{r s, v} \kappa_{t, u, w}+\kappa_{r s, w} \kappa_{t, u, v}\right) \\
+6 \sum \kappa^{a, r} \kappa^{a, u}\left(\kappa^{a, s} \kappa^{a, t}+\kappa^{a, a} \kappa^{s, t}\right) \kappa_{r s, t, u} \\
+\sum \kappa^{a, r} \kappa^{a, w}\left(\kappa^{s, t} \kappa^{a, u} \kappa^{a, v}+6 \kappa^{a, s} \kappa^{t, u} \kappa^{a, v}+3 \kappa^{a, a} \kappa^{s, u} \kappa^{t, v}\right) \kappa_{r s t} \kappa_{u, v, w} \\
-\left[\sum \kappa^{a, r} \kappa^{s, t}\left(2 \kappa_{r s, t}+\kappa_{r s t}\right)\right]\left[\sum \kappa^{a, u} \kappa^{a, v} \kappa^{a, w} \kappa_{u, v, w}\right] \\
A_{33}=\mathcal{A}_{1}+\mathcal{A}_{2}+\mathcal{A}_{3}+\mathcal{A}_{4},
\end{gathered}
$$

sendo

$$
\begin{gathered}
\mathcal{A}_{1}=9 \sum \kappa^{a, r}\left(2 \kappa^{a, a} \kappa^{s, u} \kappa^{a, t}+\kappa^{a, a} \kappa^{s, t} \kappa^{a, u}+2 \kappa^{a, s} \kappa^{a, t} \kappa^{a, u}\right) \kappa_{r s t, u} \\
\mathcal{A}_{2}=18 \sum \kappa^{a, a} \kappa^{a, r} \kappa^{s, t} \kappa^{a, u} \kappa_{r s, t u} \\
+36 \sum_{\beta, \lambda} \kappa^{a, r} \kappa^{s, t} \kappa^{a, w}\left(\kappa^{a, a} \kappa^{u, v}+\kappa^{a, u} \kappa^{a, v}\right) \kappa_{r s, v} \kappa_{t u, w} \\
+9 \sum_{\beta, \lambda} \kappa^{a, r} \kappa^{s, t}\left(2 \kappa^{a, a} \kappa^{u, w} \kappa^{a, v}+\kappa^{a, a} \kappa^{u, v} \kappa^{a, w}+2 \kappa^{a, u} \kappa^{a, v} \kappa^{a, w}\right) \kappa_{r s, w} \kappa_{t u v} \\
\mathcal{A}_{3}=6 \sum_{\sum^{a, r}} \kappa^{t, v}\left(3 \kappa^{a, a} \kappa^{s, u} \kappa^{a, w}+3 \kappa^{a, a} \kappa^{a, s} \kappa^{u, w}+4 \kappa^{a, a} \kappa^{s, w} \kappa^{a, u}+5 \kappa^{a, s} \kappa^{a, u} \kappa^{a, w}\right) \kappa_{r s t} \kappa_{u, v w} \\
+9 \sum_{\beta, \lambda} \kappa^{a, r} \kappa^{t, v}\left(2 \kappa^{a, a} \kappa^{s, u} \kappa^{a, w}+\kappa^{a, a} \kappa^{a, s} \kappa^{u, w}+2 \kappa^{a, s} \kappa^{a, u} \kappa^{a, w}\right) \kappa_{r s t} \kappa_{u v w} \\
\mathcal{A}_{4}=\sum \kappa^{a, r}\left(9 \kappa^{a, a} \kappa^{s, t} \kappa^{a, u}+6 \kappa^{a, s} \kappa^{a, t} \kappa^{a, u}\right) \kappa_{r s t u}
\end{gathered}
$$




$$
A_{22}=B_{1}-2 B_{2}+B_{3}
$$

em que

$$
B_{1}=C_{1}+C_{2}+C_{3}
$$

com

$$
\begin{aligned}
& C_{1}=4 \sum \kappa^{a, r} \kappa^{a, u}\left(\kappa^{a, a} \kappa^{s, t}+2 \kappa^{a, s} \kappa^{a, t}\right) \kappa_{r s, t u} \\
& +4 \sum \kappa^{a, r} \kappa^{a, u}\left(\kappa^{a, a} \kappa^{s, t} \kappa^{v, w}+\kappa^{a, a} \kappa^{s, w} \kappa^{t, v}+2 \kappa^{a, v} \kappa^{s, t} \kappa^{a, w}+2 \kappa^{a, s} \kappa^{t, v} \kappa^{a, w}\right. \\
& \left.+2 \kappa^{a, t} \kappa^{s, w} \kappa^{a, v}+2 \kappa^{a, t} \kappa^{s, v} \kappa^{a, w}+2 \kappa^{a, s} \kappa^{a, t} \kappa^{v, w}\right) \kappa_{r s, t} \kappa_{u v, w}, \\
& C_{2}=4 \sum \kappa^{a, r} \kappa^{a, u}\left(\kappa^{a, a} \kappa^{s, t} \kappa^{v, w}+\kappa^{a, a} \kappa^{s, w} \kappa^{t, v}+2 \kappa^{a, v} \kappa^{s, t} \kappa^{a, w}\right. \\
& +2 \kappa^{a, s} \kappa^{t, v} \kappa^{a, w}+\kappa^{a, a} \kappa^{s, v} \kappa^{t, w}+2 \kappa^{a, s} \kappa^{a, v} \kappa^{t, w} \\
& \left.+2 \kappa^{a, t} \kappa^{s, w} \kappa^{a, v}+2 \kappa^{a, t} \kappa^{s, v} \kappa^{a, w}+2 \kappa^{a, s} \kappa^{a, t} \kappa^{v, w}\right) \kappa_{r s, t} \kappa_{u v w}, \\
& C_{3}=\sum \kappa^{a, r} \kappa^{a, w}\left(\kappa^{a, a} \kappa^{s, t} \kappa^{u, v}+2 \kappa^{s, t} \kappa^{a, u} \kappa^{a, v}+2 \kappa^{a, a} \kappa^{s, u} \kappa^{t, v}\right. \\
& \left.+8 \kappa^{s, u} \kappa^{a, t} \kappa^{a, v}+2 \kappa^{a, s} \kappa^{a, t} \kappa^{u, v}\right) \kappa_{r s t} \kappa_{u v w}, \\
& B_{2}=\left[\sum \kappa^{a, r} \kappa^{s, t}\left(2 \kappa_{r s, t}+\kappa_{r s t}\right)\right]\left[\sum \kappa^{a, r}\left(\kappa^{a, a} \kappa^{s, t}+2 \kappa^{a, s} \kappa^{a, t}\right)\left(2 \kappa_{r s, t}+\kappa_{r s t}\right)\right]
\end{aligned}
$$

e

$$
B_{3}=\left[\sum \kappa^{a, r} \kappa^{s, t}\left(2 \kappa_{r s, t}+\kappa_{r s t}\right)\right]^{2} \kappa^{a, a}
$$

sendo o somatório sobre todos os $k$ parâmetros $\theta_{1}, \ldots, \theta_{k}$.

O coeficiente de curtose definido em Bowman e Shenton (1999) é expresso como

$$
\gamma_{2}\left(\hat{\theta}_{a}\right)=\left(\frac{\mu_{4}}{\left(\kappa^{a, a}\right)^{2}}-\frac{6 \sigma_{a a}}{\kappa^{a, a}}\right) / n
$$

em que $\sigma_{a a}$ é dada em (2.2).

\subsubsection{Coeficiente de curtose para a distribuição de $\hat{\boldsymbol{\beta}}$}

Seja $\boldsymbol{\theta}=\left(\boldsymbol{\beta}^{\top}, \boldsymbol{\lambda}^{\top}\right)^{\top}$ o vetor de parâmetros de dimensão $p+q \operatorname{com} \boldsymbol{\beta}=\left(\beta_{1}, \ldots, \beta_{p}\right)^{\top}$ e $\boldsymbol{\lambda}=\left(\lambda_{1}, \ldots, \lambda_{q}\right)^{\top}$ associado ao MLGH definido por (1.1) e (1.2).

Para as seções que seguem, utilizaremos a notação $[\cdots]_{d}$ para denotar a matriz diagonal 
correspondente a qualquer matriz quadrada, isto é, se $\boldsymbol{A}$ é uma matriz quadrada, então $[\boldsymbol{A}]_{d}$ será a sua matriz diagonal.

Definimos as seguintes matrizes:

$$
\begin{aligned}
& \boldsymbol{K}_{\beta}=\left(\boldsymbol{X}^{\top} \boldsymbol{W} \boldsymbol{\Phi} \boldsymbol{X}\right)^{-1}, \quad \boldsymbol{K}_{\lambda}=\left(-\boldsymbol{S}^{\top} \boldsymbol{D}_{2} \boldsymbol{\Phi}_{1}^{2} \boldsymbol{S}\right)^{-1} \\
& \boldsymbol{P}_{\beta}=\left(\boldsymbol{X}^{\top} \boldsymbol{W} \boldsymbol{\Phi} \boldsymbol{X}\right)^{-1} \boldsymbol{X}^{\top}, \quad \boldsymbol{P}_{\lambda}=\left(-\boldsymbol{S}^{\top} \boldsymbol{D}_{2} \boldsymbol{\Phi}_{1}^{2} \boldsymbol{S}\right)^{-1} \boldsymbol{S}^{\top}, \\
& \boldsymbol{Z}_{\beta}=\left\{z_{\beta \ell m}\right\}=\boldsymbol{X}\left(\boldsymbol{X}^{\top} \boldsymbol{W} \boldsymbol{\Phi} \boldsymbol{X}\right)^{-1} \boldsymbol{X}^{\top}, \quad \boldsymbol{Z}_{\lambda}=\left\{z_{\lambda \ell m}\right\}=\boldsymbol{S}\left(-\boldsymbol{S}^{\top} \boldsymbol{D}_{2} \boldsymbol{\Phi}_{1}^{2} \boldsymbol{S}\right)^{-1} \boldsymbol{S}^{\top} \\
& \boldsymbol{Z}_{\beta d}=\operatorname{diag}\left(z_{\beta 11}, \ldots, z_{\beta n n}\right), \quad \boldsymbol{Z}_{\lambda d}=\operatorname{diag}\left(z_{\lambda 11}, \ldots, z_{\lambda n n}\right), \\
& \boldsymbol{F}=\operatorname{diag}\left\{f_{1}, \ldots, f_{n}\right\}, \quad \boldsymbol{G}=\operatorname{diag}\left\{g_{1}, \ldots, g_{n}\right\}, \quad \boldsymbol{W}=\operatorname{diag}\left\{w_{1}, \ldots, w_{n}\right\}, \\
& \boldsymbol{N}=\operatorname{diag}\left\{n_{1}, \ldots, n_{n}\right\}, \quad \boldsymbol{Q}=\operatorname{diag}\left\{q_{1}, \ldots, q_{n}\right\}, \quad \boldsymbol{R}=\operatorname{diag}\left\{r_{1}, \ldots, r_{n}\right\}, \\
& \boldsymbol{T}=\operatorname{diag}\left\{t_{1}, \ldots, t_{n}\right\}, \quad \boldsymbol{B}=\operatorname{diag}\left\{b_{1}, \ldots, b_{n}\right\}, \\
& \boldsymbol{\Phi}_{1}=\operatorname{diag}\left\{\phi_{11}, \ldots, \phi_{1 n}\right\}, \quad \boldsymbol{\Phi}_{2}=\operatorname{diag}\left\{\phi_{21}, \ldots, \phi_{2 n}\right\}, \quad \boldsymbol{\Phi}_{3}=\operatorname{diag}\left\{\phi_{31}, \ldots, \phi_{3 n}\right\}, \\
& \boldsymbol{D}_{2}=\operatorname{diag}\left\{d_{21}, \ldots, d_{2 n}\right\}, \quad \boldsymbol{D}_{3}=\operatorname{diag}\left\{d_{31}, \ldots, d_{3 n}\right\} \quad \text { e } \quad \boldsymbol{D}_{4}=\operatorname{diag}\left\{d_{41}, \ldots, d_{4 n}\right\},
\end{aligned}
$$

com

$$
\begin{gathered}
f_{\ell}=\frac{1}{V_{\ell}} \frac{\mathrm{d} \mu_{\ell}}{\mathrm{d} \eta_{\ell}} \frac{\mathrm{d}^{2} \mu_{\ell}}{\mathrm{d} \eta_{\ell}^{2}}, \quad g_{\ell}=f_{\ell}-\frac{1}{V_{\ell}^{2}} \frac{\mathrm{d} V_{\ell}}{\mathrm{d} \mu_{\ell}}\left(\frac{\mathrm{d} \mu_{\ell}}{\mathrm{d} \eta_{\ell}}\right)^{3}, \quad w_{\ell}=\frac{1}{V_{\ell}}\left(\frac{\mathrm{d} \mu_{\ell}}{\mathrm{d} \eta_{\ell}}\right)^{2}, \\
\nu_{1 \ell}=\frac{1}{V_{\ell}^{3}}\left(\frac{\mathrm{d} V_{\ell}}{\mathrm{d} \mu_{\ell}}\right)^{2}\left(\frac{\mathrm{d} \mu_{\ell}}{\mathrm{d} \eta_{\ell}}\right)^{4}, \quad \nu_{2 \ell}=\frac{1}{V_{\ell}^{2}} \frac{\mathrm{d}^{2} V_{\ell}}{\mathrm{d} \mu_{\ell}^{2}}\left(\frac{\mathrm{d} \mu_{\ell}}{\mathrm{d} \eta_{\ell}}\right)^{4}, \quad \nu_{3 \ell}=\frac{1}{V_{\ell}^{2}} \frac{\mathrm{d} V_{\ell}}{\mathrm{d} \mu_{\ell}}\left(\frac{\mathrm{d} \mu_{\ell}}{\mathrm{d} \eta_{\ell}}\right)^{2} \frac{\mathrm{d}^{2} \mu_{\ell}}{\mathrm{d} \eta_{\ell}^{2}}, \\
\nu_{4 \ell}=\frac{1}{V_{\ell}}\left(\frac{\mathrm{d}^{2} \mu_{\ell}}{\mathrm{d} \eta_{\ell}^{2}}\right)^{2}, \quad \nu_{5 \ell}=\frac{1}{V_{\ell}} \frac{\mathrm{d} \mu_{\ell}}{\mathrm{d} \eta_{\ell}} \frac{\mathrm{d}^{3} \mu_{\ell}}{\mathrm{d} \eta_{\ell}{ }^{3}},
\end{gathered}
$$




$$
\begin{aligned}
& n_{\ell}=\nu_{1 \ell}+\nu_{2 \ell}, \quad q_{\ell}=\nu_{3 \ell}-\nu_{1 \ell}, \quad r_{\ell}=2 \nu_{1 \ell}-\nu_{2 \ell}-3 \nu_{3 \ell}+\nu_{5 \ell}, \\
& t_{\ell}=\nu_{1 \ell}-2 \nu_{2 \ell}+\nu_{4 \ell}, \quad b_{\ell}=-6 \nu_{1 \ell}+3 \nu_{2 \ell}+12 \nu_{3 \ell}-3 \nu_{4 \ell}-4 \nu_{5 \ell}
\end{aligned}
$$

e

$$
\phi_{k \ell}=\mathrm{d}^{k} \phi_{\ell} / \mathrm{d} \tau_{\ell}^{k} \text { e } d_{k \ell}=\mathrm{d}^{k} d\left(\phi_{\ell}\right) / \mathrm{d} \phi_{\ell}^{k}, \text { para } k=1, \ldots, 4 \text { e } \ell=1, \ldots, n
$$

Após uma longa álgebra, podemos escrever as quantidades (5.2)-(5.15), em notação matricial como

$$
\mathbf{A}_{40 \beta}=\left[\left(\boldsymbol{P}_{\beta} \odot \boldsymbol{P}_{\beta}\right) \mathbf{\Phi} \boldsymbol{N}\left(\boldsymbol{P}_{\beta} \odot \boldsymbol{P}_{\beta}\right)^{\top}\right]_{d},
$$

$$
\begin{aligned}
\mathbf{A}_{\mathbf{3 2} \beta}= & -6\left[\left(\boldsymbol{P}_{\beta} \odot \boldsymbol{P}_{\beta}\right) \boldsymbol{\Phi}(\boldsymbol{F}+\boldsymbol{G}) \boldsymbol{Z}_{\beta} \boldsymbol{\Phi}(\boldsymbol{F}-\boldsymbol{G})\left(\boldsymbol{P}_{\beta} \odot \boldsymbol{P}_{\beta}\right)^{\top}\right]_{d} \\
& +6\left[\left(\boldsymbol{P}_{\beta} \odot \boldsymbol{P}_{\beta}\right) \boldsymbol{\Phi} \boldsymbol{Q}\left(\boldsymbol{P}_{\beta} \odot \boldsymbol{P}_{\beta}\right)^{\top}\right]_{d}+6\left[\boldsymbol{K}_{\beta}^{-1}\right]_{d}\left[\boldsymbol{P}_{\beta} \boldsymbol{\Phi} \boldsymbol{Q} \boldsymbol{Z}_{\beta d} \boldsymbol{P}_{\beta}^{\top}\right]_{d} \\
& -3\left[\boldsymbol{K}_{\beta}^{-1}\right]_{d}\left[\boldsymbol{P}_{\beta} \boldsymbol{\Phi}(\boldsymbol{F}+2 \boldsymbol{G})\left(\boldsymbol{Z}_{\beta} \odot \boldsymbol{Z}_{\beta}\right) \boldsymbol{\Phi}(\boldsymbol{F}-\boldsymbol{G}) \boldsymbol{P}_{\beta}^{\top}\right]_{d} \\
& -6\left[\boldsymbol{K}_{\beta}^{-1}\right]_{d}\left[\boldsymbol{P}_{\beta} \boldsymbol{\Phi}_{1}^{2} \boldsymbol{\Phi}^{-1} \boldsymbol{W} \boldsymbol{Z}_{\lambda d} \boldsymbol{P}_{\beta}^{\top}\right]_{d} \\
& +6\left[\boldsymbol{K}_{\beta}^{-1}\right]_{d}\left[\boldsymbol{P}_{\beta} \boldsymbol{\Phi}_{1} \boldsymbol{W}\left(\boldsymbol{Z}_{\beta} \odot \boldsymbol{Z}_{\lambda}\right) \boldsymbol{\Phi}_{1} \boldsymbol{W} \boldsymbol{P}_{\beta}^{\top}\right]_{d},
\end{aligned}
$$

$$
\begin{aligned}
\mathcal{A}_{\mathbf{1} \beta}= & 27\left[\boldsymbol{K}_{\beta}^{-1}\right]_{d}\left[\boldsymbol{P}_{\beta} \boldsymbol{\Phi} \boldsymbol{R} \boldsymbol{Z}_{\beta d} \boldsymbol{P}_{\beta}^{\top}\right]_{d}+18\left[\left(\boldsymbol{P}_{\beta} \odot \boldsymbol{P}_{\beta}\right) \boldsymbol{\Phi} \boldsymbol{R}\left(\boldsymbol{P}_{\beta} \odot \boldsymbol{P}_{\beta}\right)^{\top}\right]_{d} \\
& +9\left[\boldsymbol{K}_{\beta}^{-1}\right]_{d}\left[\boldsymbol{P}_{\beta} \boldsymbol{\Phi}_{2} \boldsymbol{W} \boldsymbol{Z}_{\lambda d} \boldsymbol{P}_{\beta}^{\top}\right]_{d}
\end{aligned}
$$

$$
\begin{aligned}
\mathcal{A}_{\mathbf{2} \beta}= & 18\left[\boldsymbol{K}_{\beta}^{-1}\right]_{d}\left[\boldsymbol{P}_{\beta} \boldsymbol{\Phi} \boldsymbol{T} \boldsymbol{Z}_{\beta d} \boldsymbol{P}_{\beta}^{\top}\right]_{d} \\
& -18\left[\boldsymbol{K}_{\beta}^{-1}\right]_{d}\left[\boldsymbol{P}_{\beta} \boldsymbol{\Phi} \boldsymbol{G}\left(\boldsymbol{Z}_{\beta} \odot \boldsymbol{Z}_{\beta}\right) \boldsymbol{\Phi} \boldsymbol{F} \boldsymbol{P}_{\beta}^{\top}\right]_{d} \\
& -18\left[\left(\boldsymbol{P}_{\beta} \odot \boldsymbol{P}_{\beta}\right) \boldsymbol{\Phi} \boldsymbol{G} \boldsymbol{Z}_{\beta} \boldsymbol{\Phi} \boldsymbol{F}\left(\boldsymbol{P}_{\beta} \odot \boldsymbol{P}_{\beta}\right)^{\top}\right]_{d} \\
& -9\left[\boldsymbol{K}_{\beta}^{-1}\right]_{d}\left[\boldsymbol{P}_{\beta} \boldsymbol{\Phi} \boldsymbol{G} \operatorname{diag}\left[\boldsymbol{Z}_{\beta} \boldsymbol{Z}_{\beta d} \boldsymbol{\Phi}(\boldsymbol{F}+2 \boldsymbol{G}) \mathbf{1}\right] \boldsymbol{P}_{\beta}^{\top}\right]_{d} \\
& +18\left[\boldsymbol{K}_{\beta}^{-1}\right]_{d}\left[\boldsymbol{P}_{\beta} \boldsymbol{\Phi}_{1}^{2} \boldsymbol{\Phi}^{-1} \boldsymbol{W} \boldsymbol{Z}_{\lambda d} \boldsymbol{P}_{\beta}^{\top}\right]_{d} \\
& +18\left[\boldsymbol{K}_{\beta}^{-1}\right]_{d}\left[\boldsymbol{P}_{\beta} \boldsymbol{\Phi}_{1} \boldsymbol{W}\left(\boldsymbol{Z}_{\beta} \odot \boldsymbol{Z}_{\lambda}\right) \boldsymbol{\Phi}_{1} \boldsymbol{W} \boldsymbol{P}_{\beta}^{\top}\right]_{d} \\
& +18\left[\left(\boldsymbol{P}_{\beta} \odot \boldsymbol{P}_{\beta}\right) \boldsymbol{\Phi}_{1} \boldsymbol{W} \boldsymbol{Z}_{\lambda} \boldsymbol{\Phi}_{1} \boldsymbol{W}\left(\boldsymbol{P}_{\beta} \odot \boldsymbol{P}_{\beta}\right)^{\top}\right]_{d} \\
& -9\left[\boldsymbol{K}_{\beta}^{-1}\right]_{d}\left[\boldsymbol{P}_{\beta} \boldsymbol{\Phi}_{1} \boldsymbol{W} \operatorname{diag}\left(\boldsymbol{Z}_{\lambda} \boldsymbol{Z}_{\beta d} \boldsymbol{\Phi}_{1} \boldsymbol{W} \mathbf{1}\right) \boldsymbol{P}_{\beta}^{\top}\right]_{d} \\
& +9\left[\boldsymbol{K}_{\beta}^{-1}\right]_{d}\left[\boldsymbol{P}_{\beta} \boldsymbol{\Phi}_{1} \boldsymbol{W} \operatorname{diag}\left[\boldsymbol{Z}_{\lambda} \boldsymbol{Z}_{\lambda d}\left(\boldsymbol{D}_{\mathbf{3}} \boldsymbol{\Phi}_{1}^{3}+3 \boldsymbol{D}_{2} \boldsymbol{\Phi}_{1} \boldsymbol{\Phi}_{2}\right) \mathbf{1}\right] \boldsymbol{P}_{\beta}^{\top}\right]_{d},
\end{aligned}
$$




$$
\begin{aligned}
\mathcal{A}_{\mathbf{3} \beta}= & 6\left[\boldsymbol{K}_{\beta}^{-1}\right]_{d}\left[\boldsymbol{P}_{\beta} \boldsymbol{\Phi}(\boldsymbol{F}+2 \boldsymbol{G})\left(\boldsymbol{Z}_{\beta} \odot \boldsymbol{Z}_{\beta}\right) \boldsymbol{\Phi}(3 \boldsymbol{F}-\boldsymbol{G}) \boldsymbol{P}_{\beta}^{\top}\right]_{d} \\
& +9\left[\boldsymbol{K}_{\beta}^{-1}\right]_{d}\left[\boldsymbol{P}_{\beta} \boldsymbol{\Phi}(\boldsymbol{F}+2 \boldsymbol{G}) \operatorname{diag}\left(\boldsymbol{Z}_{\beta} \boldsymbol{Z}_{\beta d} \boldsymbol{\Phi} \boldsymbol{F} \mathbf{1}\right) \boldsymbol{P}_{\beta}^{\top}\right]_{d} \\
& +6\left[\left(\boldsymbol{P}_{\beta} \odot \boldsymbol{P}_{\beta}\right) \boldsymbol{\Phi}(\boldsymbol{F}+2 \boldsymbol{G}) \boldsymbol{Z}_{\beta} \boldsymbol{\Phi}(3 \boldsymbol{F}+\boldsymbol{G})\left(\boldsymbol{P}_{\beta} \odot \boldsymbol{P}_{\beta}\right)^{\top}\right]_{d} \\
& -30\left[\boldsymbol{K}_{\beta}^{-1}\right]_{d}\left[\boldsymbol{P}_{\beta} \boldsymbol{\Phi}_{1} \boldsymbol{W}\left(\boldsymbol{Z}_{\beta} \odot \boldsymbol{Z}_{\lambda}\right) \boldsymbol{\Phi}_{1} \boldsymbol{W} \boldsymbol{P}_{\beta}^{\top}\right]_{d} \\
& -9\left[\boldsymbol{K}_{\beta}^{-1}\right]_{d}\left[\boldsymbol{P}_{\beta} \boldsymbol{\Phi}_{1} \boldsymbol{W} \operatorname{diag}\left(\boldsymbol{Z}_{\lambda} \boldsymbol{Z}_{\beta d} \boldsymbol{\Phi}_{1} \boldsymbol{W} \mathbf{1}\right) \boldsymbol{P}_{\beta}^{\top}\right]_{d} \\
& -9\left[\boldsymbol{K}_{\beta}^{-1}\right]_{d}\left[\boldsymbol{P}_{\beta} \boldsymbol{\Phi}_{1} \boldsymbol{W} \operatorname{diag}\left[\boldsymbol{Z}_{\lambda} \boldsymbol{Z}_{\lambda d}\left(\boldsymbol{D}_{3} \boldsymbol{\Phi}_{1}^{3}+\boldsymbol{D}_{2} \boldsymbol{\Phi}_{1} \boldsymbol{\Phi}_{2}\right) \mathbf{1}\right]_{\beta}^{\top}\right]_{d} \\
& -12\left[\left(\boldsymbol{P}_{\beta} \odot \boldsymbol{P}_{\beta}\right) \boldsymbol{\Phi}_{1} \boldsymbol{W} \boldsymbol{Z}_{\lambda} \boldsymbol{\Phi}_{1} \boldsymbol{W}\left(\boldsymbol{P}_{\beta} \odot \boldsymbol{P}_{\beta}\right)^{\top}\right]_{d},
\end{aligned}
$$

$$
\begin{aligned}
\mathcal{A}_{\mathbf{4} \beta}= & 9\left[\boldsymbol{K}_{\beta}^{-1}\right]_{d}\left[\boldsymbol{P}_{\beta} \boldsymbol{\Phi} \boldsymbol{B} \boldsymbol{Z}_{\beta d} \boldsymbol{P}_{\beta}^{\top}\right]_{d}+6\left[\left(\boldsymbol{P}_{\beta} \odot \boldsymbol{P}_{\beta}\right) \boldsymbol{\Phi} \boldsymbol{B}\left(\boldsymbol{P}_{\beta} \odot \boldsymbol{P}_{\beta}\right)^{\top}\right]_{d} \\
& -9\left[\boldsymbol{K}_{\beta}^{-1}\right]_{d}\left[\boldsymbol{P}_{\beta} \boldsymbol{\Phi}_{2} \boldsymbol{W} \boldsymbol{Z}_{\lambda d} \boldsymbol{P}_{\beta}^{\top}\right]_{d}
\end{aligned}
$$

$$
\mathbf{A}_{33 \beta}=\mathcal{A}_{1 \beta}+\mathcal{A}_{\mathbf{2} \beta}+\mathcal{A}_{\mathbf{3} \beta}+\mathcal{A}_{\mathbf{4} \beta},
$$

$$
\begin{aligned}
\mathbf{C}_{\mathbf{1} \beta}= & 4\left[\boldsymbol{K}_{\beta}^{-1}\right]_{d}\left[\boldsymbol{P}_{\beta} \boldsymbol{\Phi} \boldsymbol{T} \boldsymbol{Z}_{\beta d} \boldsymbol{P}_{\beta}^{\top}\right]_{d} \\
& +8\left[\left(\boldsymbol{P}_{\beta} \odot \boldsymbol{P}_{\beta}\right) \boldsymbol{\Phi} \boldsymbol{T}\left(\boldsymbol{P}_{\beta} \odot \boldsymbol{P}_{\beta}\right)^{\top}\right]_{d} \\
& +4\left[\boldsymbol{K}_{\beta}^{-1}\right]_{d}\left[\left(\boldsymbol{P}_{\beta} \boldsymbol{Z}_{\beta d} \boldsymbol{\Phi} \boldsymbol{G} \mathbf{1}\right)\left(\boldsymbol{P}_{\beta} \boldsymbol{Z}_{\beta d} \boldsymbol{\Phi} \boldsymbol{G} \mathbf{1}\right)^{\top}\right]_{d} \\
& +4\left[\boldsymbol{K}_{\beta}^{-1}\right]_{d}\left[\boldsymbol{P}_{\beta} \boldsymbol{\Phi} \boldsymbol{G}\left(\boldsymbol{Z}_{\beta} \odot \boldsymbol{Z}_{\beta}\right) \boldsymbol{\Phi} \boldsymbol{G} \boldsymbol{P}_{\beta}^{\top}\right]_{d} \\
& +8\left[\left\{\boldsymbol{P}_{\beta} \boldsymbol{Z}_{\beta d} \boldsymbol{\Phi} \boldsymbol{G} \mathbf{1}\right\}\left\{\left(\boldsymbol{P}_{\beta} \odot \boldsymbol{P}_{\beta} \odot \boldsymbol{P}_{\beta}\right) \boldsymbol{\Phi} \boldsymbol{G} \mathbf{1}\right\}^{\top}\right]_{d} \\
& +24\left[\left(\boldsymbol{P}_{\beta} \odot \boldsymbol{P}_{\beta}\right) \boldsymbol{\Phi} \boldsymbol{G} \boldsymbol{Z}_{\beta} \boldsymbol{\Phi} \boldsymbol{G}\left(\boldsymbol{P}_{\beta} \odot \boldsymbol{P}_{\beta}\right)^{\top}\right]_{d} \\
& +8\left[\left\{\left(\boldsymbol{P}_{\beta} \odot \boldsymbol{P}_{\beta} \odot \boldsymbol{P}_{\beta}\right) \boldsymbol{\Phi} \boldsymbol{G} \mathbf{1}\right\}\left\{\boldsymbol{P}_{\beta} \boldsymbol{Z}_{\beta d} \boldsymbol{\Phi} \boldsymbol{G} \mathbf{1}\right\}^{\top}\right]_{d} \\
& +4\left[\boldsymbol{K}_{\beta}^{-1}\right]_{d}\left[\boldsymbol{P}_{\beta} \boldsymbol{\Phi}_{1}^{2} \boldsymbol{\Phi}^{-1} \boldsymbol{W} \boldsymbol{Z}_{\lambda d} \boldsymbol{P}_{\beta}^{\top}\right]_{d} \\
& +8\left[\left(\boldsymbol{P}_{\beta} \odot \boldsymbol{P}_{\beta}\right) \boldsymbol{\Phi}_{1} \boldsymbol{W} \boldsymbol{Z}_{\lambda} \boldsymbol{\Phi}_{1} \boldsymbol{W}\left(\boldsymbol{P}_{\beta} \odot \boldsymbol{P}_{\beta}\right)^{\top}\right]_{d},
\end{aligned}
$$

$$
\begin{aligned}
\mathbf{C}_{\mathbf{2} \beta}= & -4\left[\boldsymbol{K}_{\beta}^{-1}\right]_{d}\left[\left\{\boldsymbol{P}_{\beta} \boldsymbol{Z}_{\beta d} \boldsymbol{\Phi} \boldsymbol{G} \mathbf{1}\right\}\left\{\boldsymbol{P}_{\beta} \boldsymbol{Z}_{\beta d} \boldsymbol{\Phi}(\boldsymbol{F}+2 \boldsymbol{G}) \mathbf{1}\right\}^{\top}\right]_{d} \\
& -8\left[\boldsymbol{K}_{\beta}^{-1}\right]_{d}\left[\boldsymbol{P}_{\beta} \boldsymbol{\Phi} \boldsymbol{G}\left(\boldsymbol{Z}_{\beta} \odot \boldsymbol{Z}_{\beta}\right) \boldsymbol{\Phi}(\boldsymbol{F}+2 \boldsymbol{G}) \boldsymbol{P}_{\beta}^{\top}\right]_{d} \\
& -8\left[\left\{\boldsymbol{P}_{\beta} \boldsymbol{Z}_{\beta d} \boldsymbol{\Phi} \boldsymbol{G} \mathbf{1}\right\}\left\{\left(\boldsymbol{P}_{\beta} \odot \boldsymbol{P}_{\beta} \odot \boldsymbol{P}_{\beta}\right) \boldsymbol{\Phi}(\boldsymbol{F}+2 \boldsymbol{G}) \mathbf{1}\right\}^{\top}\right]_{d} \\
& -32\left[\left(\boldsymbol{P}_{\beta} \odot \boldsymbol{P}_{\beta}\right) \boldsymbol{\Phi} \boldsymbol{G} \boldsymbol{Z}_{\beta} \boldsymbol{\Phi}(\boldsymbol{F}+2 \boldsymbol{G})\left(\boldsymbol{P}_{\beta} \odot \boldsymbol{P}_{\beta}\right)^{\top}\right]_{d} \\
& -8\left[\left\{\left(\boldsymbol{P}_{\beta} \odot \boldsymbol{P}_{\beta} \odot \boldsymbol{P}_{\beta}\right) \boldsymbol{\Phi} \boldsymbol{G} \mathbf{1}\right\}\left\{\boldsymbol{P}_{\beta} \boldsymbol{Z}_{\beta d} \boldsymbol{\Phi}(\boldsymbol{F}+2 \boldsymbol{G}) \mathbf{1}\right\}^{\top}\right]_{d} \\
& -8\left[\boldsymbol{K}_{\beta}^{-1}\right]_{d}\left[\boldsymbol{P}_{\beta} \boldsymbol{\Phi}_{1} \boldsymbol{W}\left(\boldsymbol{Z}_{\beta} \odot \boldsymbol{Z}_{\lambda}\right) \boldsymbol{\Phi}_{1} \boldsymbol{W} \boldsymbol{P}_{\beta}^{\top}\right]_{d} \\
& -16\left[\left(\boldsymbol{P}_{\beta} \odot \boldsymbol{P}_{\beta}\right) \boldsymbol{\Phi}_{1} \boldsymbol{W} \boldsymbol{Z}_{\lambda} \boldsymbol{\Phi}_{1} \boldsymbol{W}\left(\boldsymbol{P}_{\beta} \odot \boldsymbol{P}_{\beta}\right)^{\top}\right]_{d},
\end{aligned}
$$




$$
\begin{aligned}
\mathbf{C}_{\mathbf{3}}=\left[\boldsymbol{K}_{\beta}^{-1}\right]_{d}\left[\left\{\boldsymbol{P}_{\beta} \boldsymbol{Z}_{\beta d} \boldsymbol{\Phi}(\boldsymbol{F}+2 \boldsymbol{G}) \mathbf{1}\right\}\left\{\boldsymbol{P}_{\beta} \boldsymbol{Z}_{\beta d} \boldsymbol{\Phi}(\boldsymbol{F}+2 \boldsymbol{G}) \mathbf{1}\right\}^{\top}\right]_{d} \\
+2\left[\left\{\boldsymbol{P}_{\beta} \boldsymbol{Z}_{\beta d} \boldsymbol{\Phi}(\boldsymbol{F}+2 \boldsymbol{G}) \mathbf{1}\right\}\left\{\left(\boldsymbol{P}_{\beta} \odot \boldsymbol{P}_{\beta} \odot \boldsymbol{P}_{\beta}\right) \boldsymbol{\Phi}(\boldsymbol{F}+2 \boldsymbol{G}) \mathbf{1}\right\}^{\top}\right]_{d} \\
+2\left[\boldsymbol{K}_{\beta}^{-1}\right]_{d}\left[\boldsymbol{P}_{\beta} \boldsymbol{\Phi}(\boldsymbol{F}+2 \boldsymbol{G})\left(\boldsymbol{Z}_{\beta} \odot \boldsymbol{Z}_{\beta}\right) \boldsymbol{\Phi}(\boldsymbol{F}+2 \boldsymbol{G}) \boldsymbol{P}_{\beta}^{\top}\right]_{d} \\
+8\left[\left(\boldsymbol{P}_{\beta} \odot \boldsymbol{P}_{\beta}\right) \boldsymbol{\Phi}(\boldsymbol{F}+2 \boldsymbol{G}) \boldsymbol{Z}_{\beta} \boldsymbol{\Phi}(\boldsymbol{F}+2 \boldsymbol{G})\left(\boldsymbol{P}_{\beta} \odot \boldsymbol{P}_{\beta}\right)^{\top}\right]_{d} \\
+2\left[\left\{\left(\boldsymbol{P}_{\beta} \odot \boldsymbol{P}_{\beta} \odot \boldsymbol{P}_{\beta}\right) \boldsymbol{\Phi}(\boldsymbol{F}+2 \boldsymbol{G}) \mathbf{1}\right\}\left\{\boldsymbol{P}_{\beta} \boldsymbol{Z}_{\beta d} \boldsymbol{\Phi}(\boldsymbol{F}+2 \boldsymbol{G}) \mathbf{1}\right\}^{\top}\right]_{d} \\
+4\left[\boldsymbol{K}_{\beta}^{-1}\right]_{d}\left[\boldsymbol{P}_{\beta} \boldsymbol{\Phi}_{1} \boldsymbol{W}\left(\boldsymbol{Z}_{\beta} \odot \boldsymbol{Z}_{\lambda}\right) \boldsymbol{\Phi}_{1} \boldsymbol{W} \boldsymbol{P}_{\beta}^{\top}\right]_{d} \\
+8\left[\left(\boldsymbol{P}_{\beta} \odot \boldsymbol{P}_{\beta}\right) \boldsymbol{\Phi}_{1} \boldsymbol{W} \boldsymbol{Z}_{\lambda} \boldsymbol{\Phi}_{1} \boldsymbol{W}\left(\boldsymbol{P}_{\beta} \odot \boldsymbol{P}_{\beta}\right)^{\top}\right]_{d}, \\
\\
\quad \mathbf{B}_{\mathbf{1} \beta}=\mathbf{C}_{\mathbf{1} \beta}+\mathbf{C}_{\mathbf{2} \beta}+\mathbf{C}_{\mathbf{3} \beta}, \\
\quad \mathbf{B}_{\mathbf{2} \beta}=\left[\boldsymbol{K}_{\beta}^{-1}\right]_{d}\left[\left(\boldsymbol{P}_{\beta} \boldsymbol{Z}_{\beta d} \boldsymbol{\Phi} \boldsymbol{F} \mathbf{1}\right)\left(\boldsymbol{P}_{\beta} \boldsymbol{Z}_{\beta d} \boldsymbol{\Phi} \boldsymbol{F} \mathbf{1}\right)^{\top}\right]_{d} \\
+2\left[\left\{\boldsymbol{P}_{\beta} \boldsymbol{Z}_{\beta d} \boldsymbol{\Phi} \boldsymbol{F} \mathbf{1}\right\}\left\{\left(\boldsymbol{P}_{\beta} \odot \boldsymbol{P}_{\beta} \odot \boldsymbol{P}_{\beta}\right) \boldsymbol{\Phi} \boldsymbol{F} \mathbf{1}\right\}^{\top}\right]_{d}, \\
\mathbf{B}_{\mathbf{3} \beta}=\left[\boldsymbol{K}_{\beta}^{-1}\right]_{d}\left[\left(\boldsymbol{P} \boldsymbol{Z}_{\beta d} \boldsymbol{\Phi} \boldsymbol{F} \mathbf{1}\right)\left(\boldsymbol{P}_{\beta} \boldsymbol{Z}_{\beta d} \boldsymbol{\Phi} \boldsymbol{F} \mathbf{1}\right)^{\top}\right]_{d}
\end{aligned}
$$

e

$$
\mathbf{A}_{22 \beta}=\mathbf{B}_{\mathbf{1} \beta}-2 \mathbf{B}_{\mathbf{2} \beta}+\mathbf{B}_{\mathbf{3} \beta}
$$

Portanto a expressão (5.1) pode ser escrita em notação matrical como

$$
\mu_{4}(\hat{\boldsymbol{\beta}})=\mathbf{A}_{\mathbf{4 0} \beta}+2 \mathbf{A}_{\mathbf{3 2} \beta}+\frac{2}{3} \mathbf{A}_{\mathbf{3 3} \beta}+\frac{3}{2} \mathbf{A}_{\mathbf{2 2} \beta}
$$

em que as matrizes $\mathbf{A}_{\mathbf{4 0} \beta}, \mathbf{A}_{\mathbf{3 2} \beta}, \mathbf{A}_{\mathbf{3 3} \beta}$ e $\mathbf{A}_{\mathbf{2 2} \beta}$ são dadas em (5.17) - (5.30).

Logo, escrevendo (5.16) em notação matricial, temos que o coeficiente de curtose de ordem $n^{-1}$ para a distribuição do EMV de $\boldsymbol{\beta}$ é dado por

$$
\gamma_{2}(\hat{\boldsymbol{\beta}})=(1 / n)\left\{\mu_{4}(\hat{\boldsymbol{\beta}})\left(\left[\boldsymbol{K}_{\beta}^{-1}\right]_{d}\right)^{-2}-6\left[\boldsymbol{\Sigma}_{\beta \beta}\right]_{d}\left(\left[\boldsymbol{K}_{\beta}^{-1}\right]_{d}\right)^{-1}\right\} \mathbf{1}_{p},
$$

em que $\mu_{4}(\hat{\boldsymbol{\beta}})$ é dado em $(5.31), \boldsymbol{K}_{\beta}$ é dado em (1.4), $\boldsymbol{\Sigma}_{\beta \beta}$ é dada pela soma das expressões (2.9)-(2.11) e $\mathbf{1}_{p}$ é um vetor de uns de dimensão $p \times 1$. 


\subsubsection{Coeficiente de curtose para a distribuição de $\hat{\lambda}$}

Novamente, após uma longa álgebra, podemos escrever as quantidades (5.2)-(5.15), em notação matricial como

$$
\mathbf{A}_{\mathbf{4 0 \lambda}}=-\left[\left(\boldsymbol{P}_{\lambda} \odot \boldsymbol{P}_{\lambda}\right) \boldsymbol{D}_{4} \boldsymbol{\Phi}_{1}^{4}\left(\boldsymbol{P}_{\lambda} \odot \boldsymbol{P}_{\lambda}\right)^{\top}\right]_{d}
$$

$$
\begin{aligned}
\mathbf{A}_{\mathbf{3 2 \lambda}}= & -6\left[\left(\boldsymbol{P}_{\lambda} \odot \boldsymbol{P}_{\lambda}\right)\left(\boldsymbol{D}_{3} \boldsymbol{\Phi}_{1}^{3}+2 \boldsymbol{D}_{2} \boldsymbol{\Phi}_{1} \boldsymbol{\Phi}_{2}\right) \boldsymbol{Z}_{\lambda} \boldsymbol{D}_{3} \boldsymbol{\Phi}_{1}^{3}\left(\boldsymbol{P}_{\lambda} \odot \boldsymbol{P}_{\lambda}\right)^{\top}\right]_{d} \\
& -6\left[\left(\boldsymbol{P}_{\lambda} \odot \boldsymbol{P}_{\lambda}\right) \boldsymbol{D}_{3} \boldsymbol{\Phi}_{1}^{2} \boldsymbol{\Phi}_{2}\left(\boldsymbol{P}_{\lambda} \odot \boldsymbol{P}_{\lambda}\right)^{\top}\right]_{d}-6\left[\boldsymbol{K}_{\lambda}^{-1}\right]_{d}\left[\boldsymbol{P}_{\lambda} \boldsymbol{D}_{3} \boldsymbol{\Phi}_{1}^{2} \boldsymbol{\Phi}_{2} \boldsymbol{Z}_{\lambda d} \boldsymbol{P}_{\lambda}^{\top}\right]_{d} \\
& -3\left[\boldsymbol{K}_{\lambda}^{-1}\right]_{d}\left[\boldsymbol{P}_{\lambda}\left(\boldsymbol{D}_{3} \boldsymbol{\Phi}_{1}^{3}+3 \boldsymbol{D}_{2} \boldsymbol{\Phi}_{1} \boldsymbol{\Phi}_{2}\right)\left(\boldsymbol{Z}_{\lambda} \odot \boldsymbol{Z}_{\lambda}\right) \boldsymbol{D}_{3} \boldsymbol{\Phi}_{1}^{3} \boldsymbol{P}_{\lambda}^{\top}\right]_{d} \\
& -6\left[\boldsymbol{K}_{\lambda}^{-1}\right]_{d}\left[\boldsymbol{P}_{\lambda} \boldsymbol{\Phi}_{1}^{2} \boldsymbol{\Phi}^{-1} \boldsymbol{W} \boldsymbol{Z}_{\beta d} \boldsymbol{P}_{\lambda}^{\top}\right]_{d} \\
& +3\left[\boldsymbol{K}_{\lambda}^{-1}\right]_{d}\left[\boldsymbol{P}_{\lambda} \boldsymbol{\Phi}_{1} \boldsymbol{W}\left(\boldsymbol{Z}_{\beta} \odot \boldsymbol{Z}_{\beta}\right) \boldsymbol{\Phi}_{1} \boldsymbol{W} \boldsymbol{P}_{\lambda}^{\top}\right]_{d}
\end{aligned}
$$

$$
\begin{aligned}
\mathcal{A}_{\mathbf{1} \lambda}= & -27\left[\boldsymbol{K}_{\lambda}^{-1}\right]_{d}\left[\boldsymbol{P}_{\lambda} \boldsymbol{D}_{2} \boldsymbol{\Phi}_{1} \boldsymbol{\Phi}_{3} \boldsymbol{Z}_{\lambda d} \boldsymbol{P}_{\lambda}^{\top}\right]_{d}-18\left[\left(\boldsymbol{P}_{\lambda} \odot \boldsymbol{P}_{\lambda}\right) \boldsymbol{D}_{2} \boldsymbol{\Phi}_{1} \boldsymbol{\Phi}_{3}\left(\boldsymbol{P}_{\lambda} \odot \boldsymbol{P}_{\lambda}\right)^{\top}\right]_{d} \\
& +18\left[\boldsymbol{K}_{\lambda}^{-1}\right]_{d}\left[\boldsymbol{P}_{\lambda} \boldsymbol{\Phi}_{2} \boldsymbol{W} \boldsymbol{Z}_{\beta d} \boldsymbol{P}_{\lambda}^{\top}\right]_{d}
\end{aligned}
$$

$$
\begin{aligned}
\mathcal{A}_{\mathbf{2} \lambda}= & -18\left[\boldsymbol{K}_{\lambda}^{-1}\right]_{d}\left[\boldsymbol{P}_{\lambda} \boldsymbol{D}_{2} \boldsymbol{\Phi}_{2}^{2} \boldsymbol{Z}_{\lambda d} \boldsymbol{P}_{\lambda}^{\top}\right]_{d} \\
& -18\left[\boldsymbol{K}_{\lambda}^{-1}\right]_{d}\left[\boldsymbol{P}_{\lambda} \boldsymbol{D}_{2} \boldsymbol{\Phi}_{1} \boldsymbol{\Phi}_{2}\left(\boldsymbol{Z}_{\lambda} \odot \boldsymbol{Z}_{\lambda}\right)\left(\boldsymbol{D}_{3} \boldsymbol{\Phi}_{1}^{3}+\boldsymbol{D}_{2} \boldsymbol{\Phi}_{1} \boldsymbol{\Phi}_{2}\right) \boldsymbol{P}_{\lambda}^{\top}\right]_{d} \\
& -18\left[\left(\boldsymbol{P}_{\lambda} \odot \boldsymbol{P}_{\lambda}\right) \boldsymbol{D}_{2} \boldsymbol{\Phi}_{1} \boldsymbol{\Phi}_{2} \boldsymbol{Z}_{\lambda}\left(\boldsymbol{D}_{3} \boldsymbol{\Phi}_{1}^{3}+\boldsymbol{D}_{2} \boldsymbol{\Phi}_{\mathbf{1}} \boldsymbol{\Phi}_{2}\right)\left(\boldsymbol{P}_{\lambda} \odot \boldsymbol{P}_{\lambda}\right)^{\top}\right]_{d} \\
& -9\left[\boldsymbol{K}_{\lambda}^{-1}\right]_{d}\left[\boldsymbol{P}_{\lambda} \boldsymbol{D}_{2} \boldsymbol{\Phi}_{1} \boldsymbol{\Phi}_{2} \operatorname{diag}\left[\boldsymbol{Z}_{\lambda} \boldsymbol{Z}_{\lambda d}\left(\boldsymbol{D}_{3} \boldsymbol{\Phi}_{1}^{3}+3 \boldsymbol{D}_{2} \boldsymbol{\Phi}_{1} \boldsymbol{\Phi}_{2}\right) \mathbf{1}\right] \boldsymbol{P}_{\lambda}^{\top}\right]_{d} \\
& +18\left[\boldsymbol{K}_{\lambda}^{-1}\right]_{d}\left[\boldsymbol{P}_{\lambda} \boldsymbol{\Phi}_{1}^{2} \boldsymbol{\Phi}^{-1} \boldsymbol{W} \boldsymbol{Z}_{\beta d} \boldsymbol{P}_{\lambda}^{\top}\right]_{d} \\
& -18\left[\boldsymbol{K}_{\lambda}^{-1}\right]_{d}\left[\boldsymbol{P}_{\lambda} \boldsymbol{\Phi}_{1} \boldsymbol{W}\left(\boldsymbol{Z}_{\beta} \odot \boldsymbol{Z}_{\beta}\right) \boldsymbol{\Phi}_{1} \boldsymbol{W} \boldsymbol{P}_{\lambda}^{\top}\right]_{d} \\
& +9\left[\boldsymbol{K}_{\lambda}^{-1}\right]_{d}\left[\boldsymbol{P}_{\lambda} \boldsymbol{D}_{2} \boldsymbol{\Phi}_{1} \boldsymbol{\Phi}_{2} \operatorname{diag}\left[\boldsymbol{Z}_{\lambda} \boldsymbol{Z}_{\beta d} \boldsymbol{\Phi}_{1} \boldsymbol{W} \mathbf{1}\right] \boldsymbol{P}_{\lambda}^{\top}\right]_{d},
\end{aligned}
$$

$$
\begin{aligned}
\mathcal{A}_{\mathbf{3} \lambda}= & 6\left[\boldsymbol{K}_{\lambda}^{-1}\right]_{d}\left[\boldsymbol{P}_{\lambda}\left(\boldsymbol{D}_{3} \boldsymbol{\Phi}_{1}^{3}+3 \boldsymbol{D}_{2} \boldsymbol{\Phi}_{1} \boldsymbol{\Phi}_{2}\right)\left(\boldsymbol{Z}_{\lambda} \odot \boldsymbol{Z}_{\lambda}\right)\left(3 \boldsymbol{D}_{3} \boldsymbol{\Phi}_{1}^{3}+2 \boldsymbol{D}_{2} \boldsymbol{\Phi}_{1} \boldsymbol{\Phi}_{2}\right) \boldsymbol{P}_{\lambda}^{\top}\right]_{d} \\
& +9\left[\boldsymbol{K}_{\lambda}^{-1}\right]_{d}\left[\boldsymbol{P}_{\lambda}\left(\boldsymbol{D}_{3} \boldsymbol{\Phi}_{1}^{3}+3 \boldsymbol{D}_{2} \boldsymbol{\Phi}_{1} \boldsymbol{\Phi}_{2}\right) \operatorname{diag}\left[\boldsymbol{Z}_{\lambda} \boldsymbol{Z}_{\lambda d}\left(\boldsymbol{D}_{3} \boldsymbol{\Phi}_{1}^{3}+\boldsymbol{D}_{2} \boldsymbol{\Phi}_{1} \boldsymbol{\Phi}_{2}\right) \mathbf{1}\right] \boldsymbol{P}_{\lambda}^{\top}\right]_{d} \\
& +9\left[\boldsymbol{K}_{\lambda}^{-1}\right]_{d}\left[\boldsymbol{P}_{\lambda}\left(\boldsymbol{D}_{3} \boldsymbol{\Phi}_{1}^{3}+3 \boldsymbol{D}_{2} \boldsymbol{\Phi}_{1} \boldsymbol{\Phi}_{2}\right) \operatorname{diag}\left(\boldsymbol{Z}_{\lambda} \boldsymbol{Z}_{\beta d} \boldsymbol{\Phi}_{1} \boldsymbol{W} \mathbf{1}\right) \boldsymbol{P}_{\lambda}^{\top}\right]_{d} \\
& +6\left[\left(\boldsymbol{P}_{\lambda} \odot \boldsymbol{P}_{\lambda}\right)\left(\boldsymbol{D}_{3} \boldsymbol{\Phi}_{1}^{3}+3 \boldsymbol{D}_{2} \boldsymbol{\Phi}_{1} \boldsymbol{\Phi}_{2}\right) \boldsymbol{Z}_{\lambda}\left(3 \boldsymbol{D}_{3} \boldsymbol{\Phi}_{1}^{3}+4 \boldsymbol{D}_{2} \boldsymbol{\Phi}_{1} \boldsymbol{\Phi}_{2}\right)\left(\boldsymbol{P}_{\lambda} \odot \boldsymbol{P}_{\lambda}\right)^{\top}\right]_{d}, \\
\mathcal{A}_{4 \lambda}= & 9\left[\boldsymbol{K}_{\lambda}^{-1}\right]_{d}\left[\boldsymbol{P}_{\lambda}\left(\boldsymbol{D}_{4} \boldsymbol{\Phi}_{1}^{4}+6 \boldsymbol{D}_{3} \boldsymbol{\Phi}_{1}^{2} \boldsymbol{\Phi}_{2}+3 \boldsymbol{D}_{2} \boldsymbol{\Phi}_{2}^{2}+4 \boldsymbol{D}_{2} \boldsymbol{\Phi}_{1} \boldsymbol{\Phi}_{3}\right) \boldsymbol{Z}_{\lambda d} \boldsymbol{P}_{\lambda}^{\top}\right]_{d} \\
& +6\left[\left(\boldsymbol{P}_{\lambda} \odot \boldsymbol{P}_{\lambda}\right)\left(\boldsymbol{D}_{4} \boldsymbol{\Phi}_{1}^{4}+6 \boldsymbol{D}_{3} \boldsymbol{\Phi}_{1}^{2} \boldsymbol{\Phi}_{2}+3 \boldsymbol{D}_{2} \boldsymbol{\Phi}_{2}^{2}+4 \boldsymbol{D}_{2} \boldsymbol{\Phi}_{1} \boldsymbol{\Phi}_{3}\right)\left(\boldsymbol{P}_{\lambda} \odot \boldsymbol{P}_{\lambda}\right)^{\top}\right]_{d} \\
& -9\left[\boldsymbol{K}_{\lambda}^{-1}\right]_{d}\left[\boldsymbol{P}_{\lambda} \boldsymbol{\Phi}_{2} \boldsymbol{W} \boldsymbol{Z}_{\beta d} \boldsymbol{P}_{\lambda}^{\top}\right]_{d},
\end{aligned}
$$




$$
\mathrm{A}_{33 \lambda}=\mathcal{A}_{1 \lambda}+\mathcal{A}_{2 \lambda}+\mathcal{A}_{3 \lambda}+\mathcal{A}_{4 \lambda},
$$

$$
\begin{aligned}
\mathbf{C}_{\boldsymbol{1} \lambda}= & -4\left[\boldsymbol{K}_{\lambda}^{-1}\right]_{d}\left[\boldsymbol{P}_{\lambda} \boldsymbol{D}_{2} \boldsymbol{\Phi}_{2}^{2} \boldsymbol{Z}_{\lambda d} \boldsymbol{P}_{\lambda}^{\top}\right]_{d} \\
& -8\left[\left(\boldsymbol{P}_{\lambda} \odot \boldsymbol{P}_{\lambda}\right) \boldsymbol{D}_{2} \boldsymbol{\Phi}_{2}^{2}\left(\boldsymbol{P}_{\lambda} \odot \boldsymbol{P}_{\lambda}\right)^{\top}\right]_{d} \\
& +4\left[\boldsymbol{K}_{\lambda}^{-1}\right]_{d}\left[\left(\boldsymbol{P}_{\lambda} \boldsymbol{Z}_{\lambda d} \boldsymbol{D}_{2} \boldsymbol{\Phi}_{1} \boldsymbol{\Phi}_{2} \mathbf{1}\right)\left(\boldsymbol{P}_{\lambda} \boldsymbol{Z}_{\lambda d} \boldsymbol{D}_{2} \boldsymbol{\Phi}_{1} \boldsymbol{\Phi}_{2} \mathbf{1}\right)^{\top}\right]_{d} \\
& +4\left[\boldsymbol{K}_{\lambda}^{-1}\right]_{d}\left[\boldsymbol{P}_{\lambda} \boldsymbol{D}_{2} \boldsymbol{\Phi}_{1} \boldsymbol{\Phi}_{2}\left(\boldsymbol{Z}_{\lambda} \odot \boldsymbol{Z}_{\lambda}\right) \boldsymbol{D}_{2} \boldsymbol{\Phi}_{1} \boldsymbol{\Phi}_{2} \boldsymbol{P}_{\lambda}^{\top}\right]_{d} \\
& +24\left[\left(\boldsymbol{P}_{\lambda} \odot \boldsymbol{P}_{\lambda}\right) \boldsymbol{D}_{2} \boldsymbol{\Phi}_{1} \boldsymbol{\Phi}_{2} \boldsymbol{Z}_{\lambda} \boldsymbol{D}_{2} \boldsymbol{\Phi}_{1} \boldsymbol{\Phi}_{2}\left(\boldsymbol{P}_{\lambda} \odot \boldsymbol{P}_{\lambda}\right)^{\top}\right]_{d} \\
& +8\left[\left\{\boldsymbol{P}_{\lambda} \boldsymbol{Z}_{\lambda d} \boldsymbol{D}_{2} \boldsymbol{\Phi}_{1} \boldsymbol{\Phi}_{2} \mathbf{1}\right\}\left\{\left(\boldsymbol{P}_{\lambda} \odot \boldsymbol{P}_{\lambda} \odot \boldsymbol{P}_{\lambda}\right) \boldsymbol{D}_{2} \boldsymbol{\Phi}_{1} \boldsymbol{\Phi}_{2} \mathbf{1}\right\}^{\top}\right]_{d} \\
& +8\left[\left\{\left(\boldsymbol{P}_{\lambda} \odot \boldsymbol{P}_{\lambda} \odot \boldsymbol{P}_{\lambda}\right) \boldsymbol{D}_{2} \boldsymbol{\Phi}_{1} \boldsymbol{\Phi}_{2} \mathbf{1}\right\}\left\{\boldsymbol{P}_{\lambda} \boldsymbol{Z}_{\lambda d} \boldsymbol{D}_{2} \boldsymbol{\Phi}_{1} \boldsymbol{\Phi}_{2} \mathbf{1}\right\}^{\top}\right]_{d} \\
& +4\left[\boldsymbol{K}_{\lambda}^{-1}\right]_{d}\left[\boldsymbol{P}_{\lambda} \boldsymbol{\Phi}_{1}^{2} \boldsymbol{\Phi}^{-1} \boldsymbol{W} \boldsymbol{Z}_{\beta d} \boldsymbol{P}_{\lambda}^{\top}\right]_{d} \\
& -4\left[\boldsymbol{K}_{\lambda}^{-1}\right]_{d}\left[\left(\boldsymbol{P}_{\lambda} \boldsymbol{Z}_{\lambda d} \boldsymbol{D}_{2} \boldsymbol{\Phi}_{1} \boldsymbol{\Phi}_{2} \mathbf{1}\right)\left(\boldsymbol{P}_{\lambda} \boldsymbol{Z}_{\beta d} \boldsymbol{\Phi}_{1} \boldsymbol{W} \mathbf{1}\right)^{\top}\right]_{d} \\
& -4\left[\boldsymbol{K}_{\lambda}^{-1}\right]_{d}\left[\left(\boldsymbol{P}_{\lambda} \boldsymbol{Z}_{\beta d} \boldsymbol{\Phi}_{1} \boldsymbol{W} \mathbf{1}\right)\left(\boldsymbol{P}_{\lambda} \boldsymbol{Z}_{\lambda d} \boldsymbol{D}_{2} \boldsymbol{\Phi}_{1} \boldsymbol{\Phi}_{2} \mathbf{1}\right)^{\top}\right]_{d} \\
& +4\left[\boldsymbol{K}_{\lambda}^{-1}\right]_{d}\left[\left(\boldsymbol{P}_{\lambda} \boldsymbol{Z}_{\beta d} \boldsymbol{\Phi}_{1} \boldsymbol{W} \mathbf{1}\right)\left(\boldsymbol{P}_{\lambda} \boldsymbol{Z}_{\beta d} \boldsymbol{\Phi}_{1} \boldsymbol{W} \mathbf{1}\right)^{\top}\right]_{d} \\
& +4\left[\boldsymbol{K}_{\lambda}^{-1}\right]_{d}\left[\boldsymbol{P}_{\lambda} \boldsymbol{\Phi}_{1} \boldsymbol{W}\left(\boldsymbol{Z}_{\beta} \odot \boldsymbol{Z}_{\beta}\right) \boldsymbol{\Phi}_{1} \boldsymbol{W} \boldsymbol{P}_{\lambda}^{\top}\right]_{d} \\
& -8\left[\left\{\boldsymbol{P}_{\lambda} \boldsymbol{Z}_{\beta d} \boldsymbol{\Phi}_{1} \boldsymbol{W} \mathbf{1}\right\}\left\{\left(\boldsymbol{P}_{\lambda} \odot \boldsymbol{P}_{\lambda} \odot \boldsymbol{P}_{\lambda}\right) \boldsymbol{D}_{2} \boldsymbol{\Phi}_{1} \boldsymbol{\Phi}_{2} \mathbf{1}\right\}^{\top}\right]_{d} \\
& -8\left[\left\{\left(\boldsymbol{P}_{\lambda} \odot \boldsymbol{P}_{\lambda} \odot \boldsymbol{P}_{\lambda}\right) \boldsymbol{D}_{2} \boldsymbol{\Phi}_{1} \boldsymbol{\Phi}_{2} \mathbf{1}\right\}\left\{\boldsymbol{P}_{\lambda} \boldsymbol{Z}_{\beta d} \boldsymbol{\Phi}_{1} \boldsymbol{W} \mathbf{1}\right\}^{\top}\right]_{d}
\end{aligned}
$$

$$
\begin{aligned}
\mathbf{C}_{\mathbf{2} \lambda}= & -4\left[\boldsymbol{K}_{\lambda}^{-1}\right]_{d}\left[\left\{\boldsymbol{P}_{\lambda} \boldsymbol{Z}_{\lambda d} \boldsymbol{D}_{2} \boldsymbol{\Phi}_{1} \boldsymbol{\Phi}_{2} \mathbf{1}\right\}\left\{\boldsymbol{P}_{\lambda} \boldsymbol{Z}_{\lambda d}\left(\boldsymbol{D}_{3} \boldsymbol{\Phi}_{1}^{3}+3 \boldsymbol{D}_{2} \boldsymbol{\Phi}_{1} \boldsymbol{\Phi}_{2}\right) \mathbf{1}\right\}^{\top}\right]_{d} \\
& -8\left[\boldsymbol{K}_{\lambda}^{-1}\right]_{d}\left[\boldsymbol{P}_{\lambda} \boldsymbol{D}_{2} \boldsymbol{\Phi}_{1} \boldsymbol{\Phi}_{2}\left(\boldsymbol{Z}_{\lambda} \odot \boldsymbol{Z}_{\lambda}\right)\left(\boldsymbol{D}_{3} \boldsymbol{\Phi}_{1}^{3}+3 \boldsymbol{D}_{2} \boldsymbol{\Phi}_{1} \boldsymbol{\Phi}_{2}\right) \boldsymbol{P}_{\lambda}^{\top}\right]_{d} \\
& -8\left[\left\{\boldsymbol{P}_{\lambda} \boldsymbol{Z}_{\lambda d} \boldsymbol{D}_{2} \boldsymbol{\Phi}_{1} \boldsymbol{\Phi}_{2} \mathbf{1}\right\}\left\{\left(\boldsymbol{P}_{\lambda} \odot \boldsymbol{P}_{\lambda} \odot \boldsymbol{P}_{\lambda}\right)\left(\boldsymbol{D}_{3} \boldsymbol{\Phi}_{1}^{3}+3 \boldsymbol{D}_{2} \boldsymbol{\Phi}_{1} \boldsymbol{\Phi}_{2}\right)\right\}^{\top}\right]_{d} \\
& -8\left[\left\{\left(\boldsymbol{P}_{\lambda} \odot \boldsymbol{P}_{\lambda} \odot \boldsymbol{P}_{\lambda}\right) \boldsymbol{D}_{2} \boldsymbol{\Phi}_{1} \boldsymbol{\Phi}_{2} \mathbf{1}\right\}\left\{\boldsymbol{P}_{\lambda} \boldsymbol{Z}_{\lambda d}\left(\boldsymbol{D}_{3} \boldsymbol{\Phi}_{1}^{3}+3 \boldsymbol{D}_{2} \boldsymbol{\Phi}_{1} \boldsymbol{\Phi}_{2}\right) \mathbf{1}\right\}^{\top}\right]_{d} \\
& -32\left[\left(\boldsymbol{P}_{\lambda} \odot \boldsymbol{P}_{\lambda}\right) \boldsymbol{D}_{2} \boldsymbol{\Phi}_{1} \boldsymbol{\Phi}_{2} \boldsymbol{Z}_{\lambda}\left(\boldsymbol{D}_{3} \boldsymbol{\Phi}_{1}^{3}+3 \boldsymbol{D}_{2} \boldsymbol{\Phi}_{1} \boldsymbol{\Phi}_{2}\right)\left(\boldsymbol{P}_{\lambda} \odot \boldsymbol{P}_{\lambda}\right)^{\top}\right]_{d} \\
& +4\left[\boldsymbol{K}_{\lambda}^{-1}\right]_{d}\left[\left(\boldsymbol{P}_{\lambda} \boldsymbol{Z}_{\lambda d} \boldsymbol{D}_{2} \boldsymbol{\Phi}_{1} \boldsymbol{\Phi}_{2} \mathbf{1}\right)\left(\boldsymbol{P}_{\lambda} \boldsymbol{Z}_{\beta d} \boldsymbol{\Phi}_{1} \boldsymbol{W} \mathbf{1}\right)^{\top}\right]_{d} \\
& +4\left[\boldsymbol{K}_{\lambda}^{-1}\right]_{d}\left[\left\{\boldsymbol{P}_{\lambda} \boldsymbol{Z}_{\beta d} \boldsymbol{\Phi}_{1} \boldsymbol{W} \mathbf{1}\right\}\left\{\boldsymbol{P}_{\lambda} \boldsymbol{Z}_{\lambda d}\left(\boldsymbol{D}_{3} \boldsymbol{\Phi}_{1}^{3}+3 \boldsymbol{D}_{2} \boldsymbol{\Phi}_{1} \boldsymbol{\Phi}_{2}\right) \mathbf{1}\right\}^{\top}\right]_{d} \\
& -4\left[\boldsymbol{K}_{\lambda}^{-1}\right]_{d}\left[\left(\boldsymbol{P}_{\lambda} \boldsymbol{Z}_{\beta d} \boldsymbol{\Phi}_{1} \boldsymbol{W} \mathbf{1}\right)\left(\boldsymbol{P}_{\lambda} \boldsymbol{Z}_{\beta d} \boldsymbol{\Phi}_{1} \boldsymbol{W} \mathbf{1}\right)^{\top}\right]_{d} \\
& -8\left[\boldsymbol{K}_{\lambda}^{-1}\right]_{d}\left[\boldsymbol{P}_{\lambda} \boldsymbol{\Phi}_{1} \boldsymbol{W}\left(\boldsymbol{Z}_{\beta} \odot \boldsymbol{Z}_{\beta}\right) \boldsymbol{\Phi}_{1} \boldsymbol{W} \boldsymbol{P}_{\lambda}^{\top}\right]_{d} \\
& +8\left[\left\{\boldsymbol{P}_{\lambda} \boldsymbol{Z}_{\beta d} \boldsymbol{\Phi}_{1} \boldsymbol{W} 1\right\}\left\{\left(\boldsymbol{P}_{\lambda} \odot \boldsymbol{P}_{\lambda} \odot \boldsymbol{P}_{\lambda}\right)\left(\boldsymbol{D}_{3} \boldsymbol{\Phi}_{1}^{3}+3 \boldsymbol{D}_{2} \boldsymbol{\Phi}_{1} \boldsymbol{\Phi}_{2}\right) \mathbf{1}\right\}^{\top}\right]_{d} \\
& +8\left[\left\{\left(\boldsymbol{P}_{\lambda} \odot \boldsymbol{P}_{\lambda} \odot \boldsymbol{P}_{\lambda}\right) \boldsymbol{D}_{2} \boldsymbol{\Phi}_{1} \boldsymbol{\Phi}_{2} \mathbf{1}\right\}\left\{\boldsymbol{P}_{\lambda} \boldsymbol{Z}_{\beta d} \boldsymbol{\Phi}_{1} \boldsymbol{W} \mathbf{1}\right\}^{\top}\right]_{d},
\end{aligned}
$$




$$
\begin{aligned}
\mathbf{C}_{\mathbf{3} \lambda}= & {\left[\boldsymbol{K}_{\lambda}^{-1}\right]_{d}\left[\left\{\boldsymbol{P}_{\lambda} \boldsymbol{Z}_{\lambda d}\left(\boldsymbol{D}_{3} \boldsymbol{\Phi}_{1}^{3}+3 \boldsymbol{D}_{2} \boldsymbol{\Phi}_{1} \boldsymbol{\Phi}_{2}\right) \mathbf{1}\right\}\left\{\boldsymbol{P}_{\lambda} \boldsymbol{Z}_{\lambda d}\left(\boldsymbol{D}_{3} \boldsymbol{\Phi}_{1}^{3}+3 \boldsymbol{D}_{2} \boldsymbol{\Phi}_{1} \boldsymbol{\Phi}_{2}\right) \mathbf{1}\right\}^{\top}\right]_{d} } \\
& +2\left[\boldsymbol{K}_{\lambda}^{-1}\right]_{d}\left[\boldsymbol{P}_{\lambda}\left(\boldsymbol{D}_{3} \boldsymbol{\Phi}_{1}^{3}+3 \boldsymbol{D}_{2} \boldsymbol{\Phi}_{1} \boldsymbol{\Phi}_{2}\right)\left(\boldsymbol{Z}_{\lambda} \odot \boldsymbol{Z}_{\lambda}\right)\left(\boldsymbol{D}_{3} \boldsymbol{\Phi}_{1}^{3}+3 \boldsymbol{D}_{2} \boldsymbol{\Phi}_{1} \boldsymbol{\Phi}_{2}\right) \boldsymbol{P}_{\lambda}^{\top}\right]_{d} \\
& +8\left[\left(\boldsymbol{P}_{\lambda} \odot \boldsymbol{P}_{\lambda}\right)\left(\boldsymbol{D}_{3} \boldsymbol{\Phi}_{1}^{3}+3 \boldsymbol{D}_{2} \boldsymbol{\Phi}_{1} \boldsymbol{\Phi}_{2}\right) \boldsymbol{Z}_{\lambda}\left(\boldsymbol{D}_{3} \boldsymbol{\Phi}_{1}^{3}+3 \boldsymbol{D}_{2} \boldsymbol{\Phi}_{1} \boldsymbol{\Phi}_{2}\right)\left(\boldsymbol{P}_{\lambda} \odot \boldsymbol{P}_{\lambda}\right)^{\top}\right]_{d} \\
& +2\left[\left\{\boldsymbol{P}_{\lambda} \boldsymbol{Z}_{\lambda d}\left(\boldsymbol{D}_{3} \boldsymbol{\Phi}_{1}^{3}+3 \boldsymbol{D}_{2} \boldsymbol{\Phi}_{1} \boldsymbol{\Phi}_{2}\right) \mathbf{1}\right\}\left\{\left(\boldsymbol{P}_{\lambda} \odot \boldsymbol{P}_{\lambda} \odot \boldsymbol{P}_{\lambda}\right)\left(\boldsymbol{D}_{3} \boldsymbol{\Phi}_{1}^{3}+3 \boldsymbol{D}_{2} \boldsymbol{\Phi}_{1} \boldsymbol{\Phi}_{2}\right) \mathbf{1}\right\}^{\top}\right]_{d} \\
& +2\left[\left\{\left(\boldsymbol{P}_{\lambda} \odot \boldsymbol{P}_{\lambda} \odot \boldsymbol{P}_{\lambda}\right)\left(\boldsymbol{D}_{3} \boldsymbol{\Phi}_{1}^{3}+3 \boldsymbol{D}_{2} \boldsymbol{\Phi}_{1} \boldsymbol{\Phi}_{2}\right) \mathbf{1}\right\}\left\{\boldsymbol{P}_{\lambda} \boldsymbol{Z}_{\lambda d}\left(\boldsymbol{D}_{3} \boldsymbol{\Phi}_{1}^{3}+3 \boldsymbol{D}_{2} \boldsymbol{\Phi}_{1} \boldsymbol{\Phi}_{2}\right) \mathbf{1}\right\}^{\top}\right]_{d} \\
& -\left[\boldsymbol{K}_{\lambda}^{-1}\right]_{d}\left[\left\{\boldsymbol{P}_{\lambda} \boldsymbol{Z}_{\lambda d}\left(\boldsymbol{D}_{3} \boldsymbol{\Phi}_{1}^{3}+3 \boldsymbol{D}_{2} \boldsymbol{\Phi}_{1} \boldsymbol{\Phi}_{2}\right) \mathbf{1}\right\}\left\{\boldsymbol{P}_{\lambda} \boldsymbol{Z}_{\beta d} \boldsymbol{\Phi}_{1} \boldsymbol{W} \mathbf{1}\right\}^{\top}\right]_{d} \\
& -\left[\boldsymbol{K}_{\lambda}^{-1}\right]_{d}\left[\left\{\boldsymbol{P}_{\lambda} \boldsymbol{Z}_{\beta d} \boldsymbol{\Phi}_{1} \boldsymbol{W} \mathbf{1}\right\}\left\{\boldsymbol{P}_{\lambda} \boldsymbol{Z}_{\lambda d}\left(\boldsymbol{D}_{3} \boldsymbol{\Phi}_{1}^{3}+3 \boldsymbol{D}_{2} \boldsymbol{\Phi}_{1} \boldsymbol{\Phi}_{2}\right) \mathbf{1}\right\}^{\top}\right]_{d} \\
& +\left[\boldsymbol{K}_{\lambda}^{-1}\right]_{d}\left[\left(\boldsymbol{P}_{\lambda} \boldsymbol{Z}_{\beta d} \boldsymbol{\Phi}_{1} \boldsymbol{W} \mathbf{1}\right)\left(\boldsymbol{P}_{\lambda} \boldsymbol{Z}_{\beta d} \boldsymbol{\Phi}_{1} \boldsymbol{W} \mathbf{1}\right)^{\top}\right]_{d} \\
& +2\left[\boldsymbol{K}_{\lambda}^{-1}\right]_{d}\left[\boldsymbol{P}_{\lambda} \boldsymbol{\Phi}_{1} \boldsymbol{W}\left(\boldsymbol{Z}_{\beta} \odot \boldsymbol{Z}_{\beta}\right) \boldsymbol{\Phi}_{1} \boldsymbol{W} \boldsymbol{P}_{\lambda}^{\top}\right]_{d} \\
& -2\left[\left\{\boldsymbol{P}_{\lambda} \boldsymbol{Z}_{\beta d} \boldsymbol{\Phi}_{1} \boldsymbol{W}\right\}\left\{\left(\boldsymbol{P}_{\lambda} \odot \boldsymbol{P}_{\lambda} \odot \boldsymbol{P}_{\lambda}\right)\left(\boldsymbol{D}_{3} \boldsymbol{\Phi}_{1}^{3}+3 \boldsymbol{D}_{2} \boldsymbol{\Phi}_{1} \boldsymbol{\Phi}_{2}\right) \mathbf{1}\right\}^{\top}\right]_{d} \\
& -2\left[\left\{\left(\boldsymbol{P}_{\lambda} \odot \boldsymbol{P}_{\lambda} \odot \boldsymbol{P}_{\lambda}\right)\left(\boldsymbol{D}_{3} \boldsymbol{\Phi}_{1}^{3}+3 \boldsymbol{D}_{2} \boldsymbol{\Phi}_{1} \boldsymbol{\Phi}_{2}\right) \mathbf{1}\right\}\left\{\boldsymbol{P}_{\lambda} \boldsymbol{Z}_{\beta d} \boldsymbol{\Phi}_{1} \boldsymbol{W}\right\}^{\top}\right]_{d},
\end{aligned}
$$

$$
\mathbf{B}_{1 \lambda}=\mathbf{C}_{1 \lambda}+\mathbf{C}_{2 \lambda}+\mathbf{C}_{3 \lambda}
$$

$$
\begin{aligned}
\mathbf{B}_{\mathbf{2} \lambda}= & {\left[\boldsymbol{K}_{\lambda}^{-1}\right]_{d}\left[\left\{\boldsymbol{P}_{\lambda} \boldsymbol{Z}_{\lambda d}\left(\boldsymbol{D}_{\mathbf{3}} \boldsymbol{\Phi}_{1}^{3}+\boldsymbol{D}_{2} \boldsymbol{\Phi}_{1} \boldsymbol{\Phi}_{2}\right) \mathbf{1}\right\}\left\{\boldsymbol{P}_{\lambda} \boldsymbol{Z}_{\lambda d}\left(\boldsymbol{D}_{3} \boldsymbol{\Phi}_{1}^{3}+\boldsymbol{D}_{2} \boldsymbol{\Phi}_{1} \boldsymbol{\Phi}_{2}\right) \mathbf{1}\right\}^{\top}\right]_{d} } \\
& +2\left[\left\{\boldsymbol{P}_{\lambda} \boldsymbol{Z}_{\lambda d}\left(\boldsymbol{D}_{3} \boldsymbol{\Phi}_{1}^{3}+\boldsymbol{D}_{2} \boldsymbol{\Phi}_{1} \boldsymbol{\Phi}_{2}\right) \mathbf{1}\right\}\left\{\left(\boldsymbol{P}_{\lambda} \odot \boldsymbol{P}_{\lambda} \odot \boldsymbol{P}_{\lambda}\right)\left(\boldsymbol{D}_{3} \boldsymbol{\Phi}_{1}^{3}+\boldsymbol{D}_{2} \boldsymbol{\Phi}_{1} \boldsymbol{\Phi}_{2}\right) \mathbf{1}\right\}^{\top}\right]_{d} \\
& +\left[\boldsymbol{K}_{\lambda}^{-1}\right]_{d}\left[\left\{\boldsymbol{P}_{\lambda} \boldsymbol{Z}_{\lambda d}\left(\boldsymbol{D}_{3} \boldsymbol{\Phi}_{1}^{3}+\boldsymbol{D}_{2} \boldsymbol{\Phi}_{1} \boldsymbol{\Phi}_{2}\right) \mathbf{1}\right\}\left\{\boldsymbol{P}_{\lambda} \boldsymbol{Z}_{\beta d} \boldsymbol{\Phi}_{1} \boldsymbol{W} \mathbf{1}\right\}^{\top}\right]_{d} \\
& +\left[\boldsymbol{K}_{\lambda}^{-1}\right]_{d}\left[\left\{\boldsymbol{P}_{\lambda} \boldsymbol{Z}_{\beta d} \boldsymbol{\Phi}_{1} \boldsymbol{W} \mathbf{1}\right\}\left\{\boldsymbol{P}_{\lambda} \boldsymbol{Z}_{\lambda d}\left(\boldsymbol{D}_{3} \boldsymbol{\Phi}_{1}^{3}+\boldsymbol{D}_{2} \boldsymbol{\Phi}_{1} \boldsymbol{\Phi}_{2}\right) \mathbf{1}\right\}^{\top}\right]_{d} \\
& +\left[\boldsymbol{K}_{\lambda}^{-1}\right]_{d}\left[\left\{\boldsymbol{P}_{\lambda} \boldsymbol{Z}_{\beta d} \boldsymbol{\Phi}_{1} \boldsymbol{W} \mathbf{1}\right\}\left\{\boldsymbol{P}_{\lambda} \boldsymbol{Z}_{\beta d} \boldsymbol{\Phi}_{1} \boldsymbol{W} \mathbf{1}\right\}^{\top}\right]_{d} \\
& +2\left[\left\{\boldsymbol{P}_{\lambda} \boldsymbol{Z}_{\beta d} \boldsymbol{\Phi}_{1} \boldsymbol{W} \mathbf{1}\right\}\left\{\left(\boldsymbol{P}_{\lambda} \odot \boldsymbol{P}_{\lambda} \odot \boldsymbol{P}_{\lambda}\right)\left(\boldsymbol{D}_{3} \boldsymbol{\Phi}_{1}^{3}+\boldsymbol{D}_{2} \boldsymbol{\Phi}_{1} \boldsymbol{\Phi}_{2}\right) \mathbf{1}\right\}^{\top}\right]_{d},
\end{aligned}
$$

$$
\begin{aligned}
\mathbf{B}_{\mathbf{3} \lambda}= & {\left[\boldsymbol{K}_{\lambda}^{-1}\right]_{d}\left[\left\{\boldsymbol{P}_{\lambda} \boldsymbol{Z}_{\lambda d}\left(\boldsymbol{D}_{\mathbf{3}} \boldsymbol{\Phi}_{1}^{3}+\boldsymbol{D}_{2} \boldsymbol{\Phi}_{1} \boldsymbol{\Phi}_{2}\right) \mathbf{1}\right\}\left\{\boldsymbol{P}_{\lambda} \boldsymbol{Z}_{\lambda d}\left(\boldsymbol{D}_{3} \boldsymbol{\Phi}_{1}^{3}+\boldsymbol{D}_{2} \boldsymbol{\Phi}_{1} \boldsymbol{\Phi}_{2}\right) \mathbf{1}\right\}^{\top}\right]_{d} } \\
& +\left[\boldsymbol{K}_{\lambda}^{-1}\right]_{d}\left[\left\{\boldsymbol{P}_{\lambda} \boldsymbol{Z}_{\lambda d}\left(\boldsymbol{D}_{3} \boldsymbol{\Phi}_{1}^{3}+\boldsymbol{D}_{2} \boldsymbol{\Phi}_{1} \boldsymbol{\Phi}_{2}\right) \mathbf{1}\right\}\left\{\boldsymbol{P}_{\lambda} \boldsymbol{Z}_{\beta d} \boldsymbol{\Phi}_{1} \boldsymbol{W}\right\}^{\top}\right]_{d} \\
& +\left[\boldsymbol{K}_{\lambda}^{-1}\right]_{d}\left[\left\{\boldsymbol{P}_{\lambda} \boldsymbol{Z}_{\beta d} \boldsymbol{\Phi}_{1} \boldsymbol{W} \mathbf{1}\right\}\left\{\boldsymbol{P}_{\lambda} \boldsymbol{Z}_{\lambda d}\left(\boldsymbol{D}_{3} \boldsymbol{\Phi}_{1}^{3}+\boldsymbol{D}_{2} \boldsymbol{\Phi}_{1} \boldsymbol{\Phi}_{2}\right) \mathbf{1}\right\}^{\top}\right]_{d} \\
& +\left[\boldsymbol{K}_{\lambda}^{-1}\right]_{d}\left[\left\{\boldsymbol{P}_{\lambda} \boldsymbol{Z}_{\beta d} \boldsymbol{\Phi}_{1} \boldsymbol{W} \mathbf{1}\right\}\left\{\boldsymbol{P}_{\lambda} \boldsymbol{Z}_{\beta d} \boldsymbol{\Phi}_{1} \boldsymbol{W} \mathbf{1}\right\}^{\top}\right]_{d}
\end{aligned}
$$

$$
\mathbf{A}_{\mathbf{2 2} \lambda}=\mathbf{B}_{\mathbf{1 \lambda}}-2 \mathbf{B}_{\mathbf{2} \lambda}+\mathbf{B}_{\mathbf{3} \lambda}
$$

Portanto, o quarto momento central de ordem $n^{-1}$ com respeito ao parâmetro $\boldsymbol{\lambda}$ pode ser escrito em notação matrical como

$$
\mu_{4}(\hat{\boldsymbol{\lambda}})=\mathbf{A}_{\mathbf{4 0 \lambda}}+2 \mathbf{A}_{\mathbf{3 2 \lambda}}+\frac{2}{3} \mathbf{A}_{\mathbf{3 3 \lambda}}+\frac{3}{2} \mathbf{A}_{\mathbf{2 2 \lambda}}
$$


em que as matrizes $\mathbf{A}_{\mathbf{4 0} \lambda}, \mathbf{A}_{\mathbf{3 2} \lambda}, \mathbf{A}_{\mathbf{3 3} \lambda}$ e $\mathbf{A}_{\mathbf{2 2} \lambda}$ são dadas em (5.32) - (5.45).

Logo, temos que o coeficiente de curtose de ordem $n^{-1}$ da distribuição do EMV de $\boldsymbol{\lambda}$ é dado por

$$
\gamma_{2}(\hat{\boldsymbol{\lambda}})=(1 / n)\left\{\mu_{4}(\hat{\boldsymbol{\lambda}})\left(\left[\boldsymbol{K}_{\lambda}^{-1}\right]_{d}\right)^{-2}-6\left[\boldsymbol{\Sigma}_{\lambda \lambda}\right]_{d}\left(\left[\boldsymbol{K}_{\lambda}^{-1}\right]_{d}\right)^{-1}\right\} \mathbf{1}_{q}
$$

em que $\mu_{4}(\hat{\boldsymbol{\lambda}})$ é dado em $(5.46), \boldsymbol{K}_{\lambda}$ é dado em (1.4), $\boldsymbol{\Sigma}_{\lambda \lambda}$ é dada pela soma das expressões (2.15)-(2.17) e $\mathbf{1}_{q}$ é um vetor de uns de dimensão $q \times 1$.

\subsection{Casos particulares}

Consideremos o mesmo contexto utilizado na Subseção 2.1.2 em que o modelo é definido por (1.1) e (1.2) com $t_{2}(\phi)$ agora sendo definido como $t_{2}(\phi)=\boldsymbol{\tau}=\lambda \mathbf{1}$, em que $\lambda$ é um escalar e 1 é um vetor $n \times 1$ de uns. Estabelecendo a ligação identidade para a função de ligação da dispersão temos $\boldsymbol{\phi}=\boldsymbol{\tau}=\lambda \mathbf{1}$ e isto implica que $\boldsymbol{\phi}=\left(\phi_{1}, \ldots, \phi_{n}\right)^{\top}=(\lambda, \ldots, \lambda)^{\top}$, ou seja, $\phi_{\ell}=\lambda$ para todo $\ell=1, \ldots, n$. Considere $\phi_{\ell}=\phi$ para todo $\ell=1, \ldots, n$, segue portanto que $\phi=\lambda$.

Utilizando as matrizes definidas em (2.18)-(2.23) nas expressões (5.17)-(5.30) e (5.32)(5.45), obtemos

$$
\begin{gathered}
\mathbf{A}_{\mathbf{4 0} \beta}=\phi^{-3}\left[(\boldsymbol{P} \odot \boldsymbol{P}) \boldsymbol{N}(\boldsymbol{P} \odot \boldsymbol{P})^{\top}\right]_{d}, \\
\mathbf{A}_{\mathbf{3 2} \beta}=-6 \phi^{-3}\left[(\boldsymbol{P} \odot \boldsymbol{P})(\boldsymbol{F}+\boldsymbol{G}) \boldsymbol{Z}(\boldsymbol{F}-\boldsymbol{G})(\boldsymbol{P} \odot \boldsymbol{P})^{\top}\right]_{d} \\
+6 \phi^{-3}\left[(\boldsymbol{P} \odot \boldsymbol{P}) \boldsymbol{Q}(\boldsymbol{P} \odot \boldsymbol{P})^{\top}\right]_{d} \\
+6 \phi^{-3}\left[\left(\boldsymbol{X}^{\top} \boldsymbol{W} \boldsymbol{X}\right)^{-1}\right]_{d}\left[\boldsymbol{P} \boldsymbol{Q} \boldsymbol{Z}_{d} \boldsymbol{P}^{\top}\right]_{d} \\
-3 \phi^{-3}\left[\left(\boldsymbol{X}^{\top} \boldsymbol{W} \boldsymbol{X}\right)^{-1}\right]_{d}\left[\boldsymbol{P}(\boldsymbol{F}+2 \boldsymbol{G})(\boldsymbol{Z} \odot \boldsymbol{Z})(\boldsymbol{F}-\boldsymbol{G}) \boldsymbol{P}^{\top}\right]_{d}, \\
\mathcal{A}_{\mathbf{1} \beta}=27 \phi^{-3}\left[\left(\boldsymbol{X}^{\top} \boldsymbol{W} \boldsymbol{X}\right)^{-1}\right]_{d}\left[\boldsymbol{P} \boldsymbol{R} \boldsymbol{Z}_{d} \boldsymbol{P}^{\top}\right]_{d}+18 \phi^{-3}\left[(\boldsymbol{P} \odot \boldsymbol{P}) \boldsymbol{R}(\boldsymbol{P} \odot \boldsymbol{P})^{\top}\right]_{d},
\end{gathered}
$$




$$
\begin{aligned}
& \mathcal{A}_{\mathbf{2} \beta}=18 \phi^{-3}\left[\left(\boldsymbol{X}^{\top} \boldsymbol{W} \boldsymbol{X}\right)^{-1}\right]_{d}\left[\boldsymbol{P} \boldsymbol{T} \boldsymbol{Z}_{d} \boldsymbol{P}^{\top}\right]_{d} \\
& -18 \phi^{-3}\left[\left(\boldsymbol{X}^{\top} \boldsymbol{W} \boldsymbol{X}\right)^{-1}\right]_{d}\left[\boldsymbol{P G}(\boldsymbol{Z} \odot \boldsymbol{Z}) \boldsymbol{F} \boldsymbol{P}^{\top}\right]_{d} \\
& -18 \phi^{-3}\left[(\boldsymbol{P} \odot \boldsymbol{P}) \boldsymbol{G} \boldsymbol{Z} \boldsymbol{F}(\boldsymbol{P} \odot \boldsymbol{P})^{\top}\right]_{d} \\
& -9 \phi^{-3}\left[\left(\boldsymbol{X}^{\top} \boldsymbol{W} \boldsymbol{X}\right)^{-1}\right]_{d}\left[\boldsymbol{P} \boldsymbol{G} \operatorname{diag}\left[\boldsymbol{Z} \boldsymbol{Z}_{d}(\boldsymbol{F}+2 \boldsymbol{G}) \mathbf{1}\right] \boldsymbol{P}^{\top}\right]_{d} \\
& -\frac{36}{n d_{2} \phi^{4}}\left[\left(\boldsymbol{X}^{\top} \boldsymbol{W} \boldsymbol{X}\right)^{-1}\right]_{d}\left[\boldsymbol{P} \boldsymbol{W} \boldsymbol{P}^{\top}\right]_{d} \\
& -\frac{18}{n d_{2} \phi^{4}}\left[(\boldsymbol{P} \odot \boldsymbol{P}) \boldsymbol{W} \mathbf{1 1}{ }^{\top} \boldsymbol{W}(\boldsymbol{P} \odot \boldsymbol{P})^{\top}\right]_{d} \\
& +\frac{9 p}{n d_{2} \phi^{4}}\left[\left(\boldsymbol{X}^{\top} \boldsymbol{W} \boldsymbol{X}\right)^{-1}\right]_{d}\left[\boldsymbol{P} \boldsymbol{W} \boldsymbol{P}^{\top}\right]_{d} \\
& +\frac{9 d_{3}}{n d_{2}^{2} \phi^{3}}\left[\left(\boldsymbol{X}^{\top} \boldsymbol{W} \boldsymbol{X}\right)^{-1}\right]_{d}\left[\boldsymbol{P} \boldsymbol{W} \boldsymbol{P}^{\top}\right]_{d}, \\
& \mathcal{A}_{\mathbf{3} \beta}=6 \phi^{-3}\left[\left(\boldsymbol{X}^{\top} \boldsymbol{W} \boldsymbol{X}\right)^{-1}\right]_{d}\left[\boldsymbol{P}(\boldsymbol{F}+2 \boldsymbol{G})(\boldsymbol{Z} \odot \boldsymbol{Z})(3 \boldsymbol{F}-\boldsymbol{G}) \boldsymbol{P}^{\top}\right]_{d} \\
& +9 \phi^{-3}\left[\left(\boldsymbol{X}^{\top} \boldsymbol{W} \boldsymbol{X}\right)^{-1}\right]_{d}\left[\boldsymbol{P}(\boldsymbol{F}+2 \boldsymbol{G}) \operatorname{diag}\left(\boldsymbol{Z} \boldsymbol{Z}_{d} \boldsymbol{F} \mathbf{1}\right) \boldsymbol{P}^{\top}\right]_{d} \\
& +6 \phi^{-3}\left[(\boldsymbol{P} \odot \boldsymbol{P})(\boldsymbol{F}+2 \boldsymbol{G}) \boldsymbol{Z}(3 \boldsymbol{F}+\boldsymbol{G})(\boldsymbol{P} \odot \boldsymbol{P})^{\top}\right]_{d} \\
& +\frac{30}{n d_{2} \phi^{4}}\left[\left(\boldsymbol{X}^{\top} \boldsymbol{W} \boldsymbol{X}\right)^{-1}\right]_{d}\left[\boldsymbol{P} \boldsymbol{W} \boldsymbol{Z} \boldsymbol{W} \boldsymbol{P}^{\top}\right]_{d} \\
& +\frac{9 p}{n d_{2} \phi^{4}}\left[\left(\boldsymbol{X}^{\top} \boldsymbol{W} \boldsymbol{X}\right)^{-1}\right]_{d}\left[\boldsymbol{P} \boldsymbol{W} \boldsymbol{P}^{\top}\right]_{d} \\
& -\frac{9 d_{3}}{n d_{2}^{2} \phi^{3}}\left[\left(\boldsymbol{X}^{\top} \boldsymbol{W} \boldsymbol{X}\right)^{-1}\right]_{d}\left[\boldsymbol{P} \boldsymbol{W} \boldsymbol{P}^{\top}\right]_{d} \\
& +\frac{12}{n d_{2} \phi^{4}}\left[(\boldsymbol{P} \odot \boldsymbol{P}) \boldsymbol{W} \mathbf{1 1}{ }^{\top} \boldsymbol{W}(\boldsymbol{P} \odot \boldsymbol{P})^{\top}\right]_{d}, \\
& \mathcal{A}_{\mathbf{4} \beta}=9 \phi^{3}\left[\left(\boldsymbol{X}^{\top} \boldsymbol{W} \boldsymbol{X}\right)^{-1}\right]_{d}\left[\boldsymbol{P} \boldsymbol{B} \boldsymbol{Z}_{d} \boldsymbol{P}^{\top}\right]_{d}+6 \phi^{-3}\left[(\boldsymbol{P} \odot \boldsymbol{P}) \boldsymbol{B}(\boldsymbol{P} \odot \boldsymbol{P})^{\top}\right]_{d}, \\
& \mathbf{A}_{\mathbf{3 3} \beta}=\mathcal{A}_{\mathbf{1} \beta}+\mathcal{A}_{\mathbf{2} \beta}+\mathcal{A}_{\mathbf{3} \beta}+\mathcal{A}_{\mathbf{4} \beta},
\end{aligned}
$$




$$
\begin{aligned}
& \mathbf{C}_{\mathbf{1} \beta}=4 \phi^{-3}\left[\left(\boldsymbol{X}^{\top} \boldsymbol{W} \boldsymbol{X}\right)^{-1}\right]_{d}\left[\boldsymbol{P} \boldsymbol{T} \boldsymbol{Z}_{d} \boldsymbol{P}^{\top}\right]_{d} \\
& +8 \phi^{-3}\left[(\boldsymbol{P} \odot \boldsymbol{P}) \boldsymbol{T}(\boldsymbol{P} \odot \boldsymbol{P})^{\top}\right]_{d} \\
& +4 \phi^{-3}\left[\left(\boldsymbol{X}^{\top} \boldsymbol{W} \boldsymbol{X}\right)^{-1}\right]_{d}\left[\left(\boldsymbol{P} \boldsymbol{Z}_{d} \boldsymbol{G} \mathbf{1}\right)\left(\boldsymbol{P} \boldsymbol{Z}_{d} \boldsymbol{G} \mathbf{1}\right)^{\top}\right]_{d} \\
& +4 \phi^{-3}\left[\left(\boldsymbol{X}^{\top} \boldsymbol{W} \boldsymbol{X}\right)^{-1}\right]_{d}\left[\boldsymbol{P G}(\boldsymbol{Z} \odot \boldsymbol{Z}) \boldsymbol{G} \boldsymbol{P}^{\top}\right]_{d} \\
& +8 \phi^{-3}\left[\left\{\boldsymbol{P} \boldsymbol{Z}_{d} \boldsymbol{G} \mathbf{1}\right\}\{(\boldsymbol{P} \odot \boldsymbol{P} \odot \boldsymbol{P}) \boldsymbol{G} \mathbf{1}\}^{\top}\right]_{d} \\
& +24 \phi^{-3}\left[(\boldsymbol{P} \odot \boldsymbol{P}) \boldsymbol{G} \boldsymbol{Z} \boldsymbol{G}(\boldsymbol{P} \odot \boldsymbol{P})^{\top}\right]_{d} \\
& +8 \phi^{-3}\left[\{(\boldsymbol{P} \odot \boldsymbol{P} \odot \boldsymbol{P}) \boldsymbol{G} \mathbf{1}\}\left\{\boldsymbol{P} \boldsymbol{Z}_{d} \boldsymbol{G} \mathbf{1}\right\}^{\top}\right]_{d}, \\
& -\frac{4}{n d_{2} \phi^{4}}\left[\left(\boldsymbol{X}^{\top} \boldsymbol{W} \boldsymbol{X}\right)^{-1}\right]_{d}\left[\boldsymbol{P} \boldsymbol{W} \boldsymbol{P}^{\top}\right]_{d} \\
& -\frac{8}{n d_{2} \phi^{4}}\left[(\boldsymbol{P} \odot \boldsymbol{P}) \boldsymbol{W} \mathbf{1 1}{ }^{\top} \boldsymbol{W}(\boldsymbol{P} \odot \boldsymbol{P})^{\top}\right]_{d}, \\
& \mathbf{C}_{\mathbf{2} \beta}=-4 \phi^{-3}\left[\left(\boldsymbol{X}^{\top} \boldsymbol{W} \boldsymbol{X}\right)^{-1}\right]_{d}\left[\left\{\boldsymbol{P} \boldsymbol{Z}_{d} \boldsymbol{G} \mathbf{1}\right\}\left\{\boldsymbol{P} \boldsymbol{Z}_{d}(\boldsymbol{F}+2 \boldsymbol{G}) \mathbf{1}\right\}^{\top}\right]_{d} \\
& -8 \phi^{-3}\left[\left(\boldsymbol{X}^{\top} \boldsymbol{W} \boldsymbol{X}\right)^{-1}\right]_{d}\left[\boldsymbol{P G}(\boldsymbol{Z} \odot \boldsymbol{Z})(\boldsymbol{F}+2 \boldsymbol{G}) \boldsymbol{P}^{\top}\right]_{d} \\
& -8 \phi^{-3}\left[\left\{\boldsymbol{P} \boldsymbol{Z}_{d} \boldsymbol{G} \mathbf{1}\right\}\{(\boldsymbol{P} \odot \boldsymbol{P} \odot \boldsymbol{P})(\boldsymbol{F}+2 \boldsymbol{G}) \mathbf{1}\}^{\top}\right]_{d} \\
& -32 \phi^{-3}\left[(\boldsymbol{P} \odot \boldsymbol{P}) \boldsymbol{G} \boldsymbol{Z}(\boldsymbol{F}+2 \boldsymbol{G})(\boldsymbol{P} \odot \boldsymbol{P})^{\top}\right]_{d} \\
& -8 \phi^{-3}\left[\{(\boldsymbol{P} \odot \boldsymbol{P} \odot \boldsymbol{P}) \boldsymbol{G} \mathbf{1}\}\left\{\boldsymbol{P} \boldsymbol{Z}_{d}(\boldsymbol{F}+2 \boldsymbol{G}) \mathbf{1}\right\}^{\top}\right]_{d}, \\
& +\frac{8}{n d_{2} \phi^{4}}\left[\left(\boldsymbol{X}^{\top} \boldsymbol{W} \boldsymbol{X}\right)^{-1}\right]_{d}\left[\boldsymbol{P} \boldsymbol{W} \boldsymbol{Z} \boldsymbol{W} \boldsymbol{P}^{\top}\right]_{d} \\
& +\frac{16}{n d_{2} \phi^{4}}\left[(\boldsymbol{P} \odot \boldsymbol{P}) \boldsymbol{W} \mathbf{1 1}{ }^{\top} \boldsymbol{W}(\boldsymbol{P} \odot \boldsymbol{P})^{\top}\right]_{d}, \\
& \mathbf{C}_{\mathbf{3} \beta}=\phi^{-3}\left[\left(\boldsymbol{X}^{\top} \boldsymbol{W} \boldsymbol{X}\right)^{-1}\right]_{d}\left[\left\{\boldsymbol{P} \boldsymbol{Z}_{d}(\boldsymbol{F}+2 \boldsymbol{G}) \mathbf{1}\right\}\left\{\boldsymbol{P} \boldsymbol{Z}_{d}(\boldsymbol{F}+2 \boldsymbol{G}) \mathbf{1}\right\}^{\top}\right]_{d} \\
& +2 \phi^{-3}\left[\left\{\boldsymbol{P} \boldsymbol{Z}_{d}(\boldsymbol{F}+2 \boldsymbol{G}) \mathbf{1}\right\}\{(\boldsymbol{P} \odot \boldsymbol{P} \odot \boldsymbol{P})(\boldsymbol{F}+2 \boldsymbol{G}) \mathbf{1}\}^{\top}\right]_{d} \\
& +2 \phi^{-3}\left[\left(\boldsymbol{X}^{\top} \boldsymbol{W} \boldsymbol{X}\right)^{-1}\right]_{d}\left[\boldsymbol{P}(\boldsymbol{F}+2 \boldsymbol{G})(\boldsymbol{Z} \odot \boldsymbol{Z})(\boldsymbol{F}+2 \boldsymbol{G}) \boldsymbol{P}^{\top}\right]_{d} \\
& +8 \phi^{-3}\left[(\boldsymbol{P} \odot \boldsymbol{P})(\boldsymbol{F}+2 \boldsymbol{G}) \boldsymbol{Z}(\boldsymbol{F}+2 \boldsymbol{G})(\boldsymbol{P} \odot \boldsymbol{P})^{\top}\right]_{d} \\
& +2 \phi^{-3}\left[\{(\boldsymbol{P} \odot \boldsymbol{P} \odot \boldsymbol{P})(\boldsymbol{F}+2 \boldsymbol{G}) \mathbf{1}\}\left\{\boldsymbol{P} \boldsymbol{Z}_{d}(\boldsymbol{F}+2 \boldsymbol{G}) \mathbf{1}\right\}^{\top}\right]_{d} \\
& -\frac{4}{n d_{2} \phi^{4}}\left[\left(\boldsymbol{X}^{\top} \boldsymbol{W} \boldsymbol{X}\right)^{-1}\right]_{d}\left[\boldsymbol{P} \boldsymbol{W} \boldsymbol{Z} \boldsymbol{W} \boldsymbol{P}^{\top}\right]_{d} \\
& -\frac{8}{n d_{2} \phi^{4}}\left[(\boldsymbol{P} \odot \boldsymbol{P}) \boldsymbol{W} \mathbf{1 1}{ }^{\top} \boldsymbol{W}(\boldsymbol{P} \odot \boldsymbol{P})^{\top}\right]_{d}, \\
& \mathbf{B}_{\mathbf{1} \beta}=\mathbf{C}_{\mathbf{1} \beta}+\mathbf{C}_{\mathbf{2} \beta}+\mathbf{C}_{\mathbf{3} \beta},
\end{aligned}
$$




$$
\begin{aligned}
& \mathbf{B}_{\mathbf{2} \beta}=\phi^{-3}\left[\left(\boldsymbol{X}^{\top} \boldsymbol{W} \boldsymbol{X}\right)^{-1}\right]_{d}\left[\left(\boldsymbol{P} \boldsymbol{Z}_{d} \boldsymbol{F} \mathbf{1}\right)\left(\boldsymbol{P} \boldsymbol{Z}_{d} \boldsymbol{F} \mathbf{1}\right)^{\top}\right]_{d} \\
& +2 \phi^{-3}\left[\left\{\boldsymbol{P} \boldsymbol{Z}_{d} \boldsymbol{F} \mathbf{1}\right\}\{(\boldsymbol{P} \odot \boldsymbol{P} \odot \boldsymbol{P}) \boldsymbol{F} \mathbf{1}\}^{\top}\right]_{d}, \\
& \mathbf{B}_{\mathbf{3} \beta}=\phi^{-3}\left[\left(\boldsymbol{X}^{\top} \boldsymbol{W} \boldsymbol{X}\right)^{-1}\right]_{d}\left[\left(\boldsymbol{P} \boldsymbol{Z}_{d} \boldsymbol{F} \mathbf{1}\right)\left(\boldsymbol{P} \boldsymbol{Z}_{d} \boldsymbol{F} \mathbf{1}\right)^{\top}\right]_{d}, \\
& \mathbf{A}_{\mathbf{2} \beta}=\mathbf{B}_{\mathbf{1} \beta}-2 \mathbf{B}_{\mathbf{2} \beta}+\mathbf{B}_{\mathbf{3} \beta}, \\
& A_{40 \phi}=-\frac{d_{4}}{n^{3} d_{2}^{4}} \\
& A_{32 \phi}=\frac{6 d_{3}^{2}}{n^{2} d_{2}^{4}}+\frac{3 d_{3}^{2}}{n^{3} d_{2}^{5}}+\frac{6 p}{n^{3} d_{2}^{3} \phi^{2}}-\frac{3}{n^{3} d_{2}^{3} \phi^{2}} \mathbf{1}^{\top} \boldsymbol{W}(\boldsymbol{Z} \odot \boldsymbol{Z}) \boldsymbol{W} \mathbf{1}, \\
& \mathcal{A}_{1 \phi}=0, \\
& \mathcal{A}_{2 \phi}=\frac{p}{n^{2} d_{2}^{2} \phi^{2}}-\frac{1}{n^{2} d_{2}^{2} \phi^{2}} \mathbf{1}^{\top} \boldsymbol{W}(\boldsymbol{Z} \odot \boldsymbol{Z}) \boldsymbol{W} \mathbf{1}, \\
& \mathcal{A}_{3 \phi}=-\frac{9 d_{3}^{2}}{n^{3} d_{2}^{5}}+\frac{9 p d_{3}}{n^{3} d_{2}^{4} \phi} \\
& \mathcal{A}_{4 \phi}=\frac{15 d_{4}}{n^{3} d_{2}^{4}} \\
& A_{33 \phi}=\mathcal{A}_{1 \phi}+\mathcal{A}_{2 \phi}+\mathcal{A}_{3 \phi}+\mathcal{A}_{4 \phi}, \\
& C_{1 \phi}=-\frac{p}{n^{3} d_{2}^{3} \phi^{2}}(4+p)+\frac{4}{n^{3} d_{2}^{3} \phi^{2}} \mathbf{1}^{\top} \boldsymbol{W}(\boldsymbol{Z} \odot \boldsymbol{Z}) \boldsymbol{W} \mathbf{1}, \\
& C_{2 \phi}=\frac{12 p d_{3}}{n^{3} d_{2}^{4} \phi}+\frac{4 p^{2}}{n^{3} d_{2}^{3} \phi^{2}}-\frac{8}{n^{3} d_{2}^{3} \phi^{2}} \mathbf{1}^{\top} \boldsymbol{W}(\boldsymbol{Z} \odot \boldsymbol{Z}) \boldsymbol{W} \mathbf{1},
\end{aligned}
$$




$$
\begin{gathered}
C_{3 \phi}=\frac{d_{3}^{2}}{n^{3} d_{2}^{5}}-\frac{6 p d_{3}}{n^{3} d_{2}^{4} \phi}-\frac{p^{2}}{n^{3} d_{2}^{3} \phi^{2}}+\frac{2}{n^{3} d_{2}^{3} \phi^{2}} \mathbf{1}^{\top} \boldsymbol{W}(\boldsymbol{Z} \odot \boldsymbol{Z}) \boldsymbol{W} \mathbf{1} \\
B_{1 \phi}=C_{1 \phi}+C_{2 \phi}+C_{3 \phi} \\
B_{2 \phi}=-\frac{3 d_{3}^{2}}{n^{3} d_{2}^{5}}+\frac{4 p d_{3}}{n^{3} d_{2}^{4} \phi}-\frac{p^{2}}{n^{3} d_{2}^{3} \phi^{2}} \\
B_{3 \phi}=-\frac{d_{3}^{2}}{n^{3} d_{2}^{5}}+\frac{2 p d_{3}}{n^{3} d_{2}^{4} \phi}-\frac{p^{2}}{n^{3} d_{2}^{3} \phi^{2}}
\end{gathered}
$$

e

$$
A_{22 \phi}=B_{1 \phi}-2 B_{2 \phi}+B_{3 \phi}
$$

Considerando agora o parâmetro de dispersão conhecido, obtemos

$$
\begin{aligned}
& \mathbf{A}_{\mathbf{4 0} \beta}=\phi^{-3}\left[(\boldsymbol{P} \odot \boldsymbol{P}) \boldsymbol{N}(\boldsymbol{P} \odot \boldsymbol{P})^{\top}\right]_{d}, \\
& \mathbf{A}_{\mathbf{3 2} \beta}=-6 \phi^{-3}\left[(\boldsymbol{P} \odot \boldsymbol{P})(\boldsymbol{F}+\boldsymbol{G}) \boldsymbol{Z}(\boldsymbol{F}-\boldsymbol{G})(\boldsymbol{P} \odot \boldsymbol{P})^{\top}\right]_{d} \\
& +6 \phi^{-3}\left[(\boldsymbol{P} \odot \boldsymbol{P}) \boldsymbol{Q}(\boldsymbol{P} \odot \boldsymbol{P})^{\top}\right]_{d} \\
& +6 \phi^{-3}\left[\left(\boldsymbol{X}^{\top} \boldsymbol{W} \boldsymbol{X}\right)^{-1}\right]_{d}\left[\boldsymbol{P Q} \boldsymbol{Z}_{d} \boldsymbol{P}^{\top}\right]_{d} \\
& -3 \phi^{-3}\left[\left(\boldsymbol{X}^{\top} \boldsymbol{W} \boldsymbol{X}\right)^{-1}\right]_{d}\left[\boldsymbol{P}(\boldsymbol{F}+2 \boldsymbol{G})(\boldsymbol{Z} \odot \boldsymbol{Z})(\boldsymbol{F}-\boldsymbol{G}) \boldsymbol{P}^{\top}\right]_{d}, \\
& \mathcal{A}_{\mathbf{1} \beta}=27 \phi^{-3}\left[\left(\boldsymbol{X}^{\top} \boldsymbol{W} \boldsymbol{X}\right)^{-1}\right]_{d}\left[\boldsymbol{P} \boldsymbol{R} \boldsymbol{Z}_{d} \boldsymbol{P}^{\top}\right]_{d}+18 \phi^{-3}\left[(\boldsymbol{P} \odot \boldsymbol{P}) \boldsymbol{R}(\boldsymbol{P} \odot \boldsymbol{P})^{\top}\right]_{d}, \\
& \mathcal{A}_{\mathbf{2} \beta}=18 \phi^{-3}\left[\left(\boldsymbol{X}^{\top} \boldsymbol{W} \boldsymbol{X}\right)^{-1}\right]_{d}\left[\boldsymbol{P} \boldsymbol{T} \boldsymbol{Z}_{d} \boldsymbol{P}^{\top}\right]_{d} \\
& -18 \phi^{-3}\left[\left(\boldsymbol{X}^{\top} \boldsymbol{W} \boldsymbol{X}\right)^{-1}\right]_{d}\left[\boldsymbol{P G}(\boldsymbol{Z} \odot \boldsymbol{Z}) \boldsymbol{F} \boldsymbol{P}^{\top}\right]_{d} \\
& -18 \phi^{-3}\left[(\boldsymbol{P} \odot \boldsymbol{P}) \boldsymbol{G} \boldsymbol{Z} \boldsymbol{F}(\boldsymbol{P} \odot \boldsymbol{P})^{\top}\right]_{d} \\
& -9 \phi^{-3}\left[\left(\boldsymbol{X}^{\top} \boldsymbol{W} \boldsymbol{X}\right)^{-1}\right]_{d}\left[\boldsymbol{P} \boldsymbol{G} \operatorname{diag}\left[\boldsymbol{Z} \boldsymbol{Z}_{d}(\boldsymbol{F}+2 \boldsymbol{G}) \mathbf{1}\right] \boldsymbol{P}^{\top}\right]_{d}, \\
& \mathcal{A}_{\mathbf{3} \beta}=6 \phi^{-3}\left[\left(\boldsymbol{X}^{\top} \boldsymbol{W} \boldsymbol{X}\right)^{-1}\right]_{d}\left[\boldsymbol{P}(\boldsymbol{F}+2 \boldsymbol{G})(\boldsymbol{Z} \odot \boldsymbol{Z})(3 \boldsymbol{F}-\boldsymbol{G}) \boldsymbol{P}^{\top}\right]_{d} \\
& +9 \phi^{-3}\left[\left(\boldsymbol{X}^{\top} \boldsymbol{W} \boldsymbol{X}\right)^{-1}\right]_{d}\left[\boldsymbol{P}(\boldsymbol{F}+2 \boldsymbol{G}) \operatorname{diag}\left(\boldsymbol{Z} \boldsymbol{Z}_{d} \boldsymbol{F} \mathbf{1}\right) \boldsymbol{P}^{\top}\right]_{d} \\
& +6 \phi^{-3}\left[(\boldsymbol{P} \odot \boldsymbol{P})(\boldsymbol{F}+2 \boldsymbol{G}) \boldsymbol{Z}(3 \boldsymbol{F}+\boldsymbol{G})(\boldsymbol{P} \odot \boldsymbol{P})^{\top}\right]_{d},
\end{aligned}
$$




$$
\begin{aligned}
& \mathcal{A}_{\mathbf{4} \beta}=9 \phi^{3}\left[\left(\boldsymbol{X}^{\top} \boldsymbol{W} \boldsymbol{X}\right)^{-1}\right]_{d}\left[\boldsymbol{P} \boldsymbol{B} \boldsymbol{Z}_{d} \boldsymbol{P}^{\top}\right]_{d}+6 \phi^{-3}\left[(\boldsymbol{P} \odot \boldsymbol{P}) \boldsymbol{B}(\boldsymbol{P} \odot \boldsymbol{P})^{\top}\right]_{d} \\
& \mathbf{A}_{\mathbf{3 3} \beta}=\mathcal{A}_{\mathbf{1} \beta}+\mathcal{A}_{\mathbf{2} \beta}+\mathcal{A}_{\mathbf{3} \beta}+\mathcal{A}_{\mathbf{4} \beta}, \\
& \mathbf{C}_{\mathbf{1} \beta}=4 \phi^{-3}\left[\left(\boldsymbol{X}^{\top} \boldsymbol{W} \boldsymbol{X}\right)^{-1}\right]_{d}\left[\boldsymbol{P} \boldsymbol{T} \boldsymbol{Z}_{d} \boldsymbol{P}^{\top}\right]_{d} \\
& +8 \phi^{-3}\left[(\boldsymbol{P} \odot \boldsymbol{P}) \boldsymbol{T}(\boldsymbol{P} \odot \boldsymbol{P})^{\top}\right]_{d} \\
& +4 \phi^{-3}\left[\left(\boldsymbol{X}^{\top} \boldsymbol{W} \boldsymbol{X}\right)^{-1}\right]_{d}\left[\left(\boldsymbol{P} \boldsymbol{Z}_{d} \boldsymbol{G} \mathbf{1}\right)\left(\boldsymbol{P} \boldsymbol{Z}_{d} \boldsymbol{G} \mathbf{1}\right)^{\top}\right]_{d} \\
& +4 \phi^{-3}\left[\left(\boldsymbol{X}^{\top} \boldsymbol{W} \boldsymbol{X}\right)^{-1}\right]_{d}\left[\boldsymbol{P G}(\boldsymbol{Z} \odot \boldsymbol{Z}) \boldsymbol{G} \boldsymbol{P}^{\top}\right]_{d} \\
& +8 \phi^{-3}\left[\left\{\boldsymbol{P} \boldsymbol{Z}_{d} \boldsymbol{G} \mathbf{1}\right\}\{(\boldsymbol{P} \odot \boldsymbol{P} \odot \boldsymbol{P}) \boldsymbol{G} \mathbf{1}\}^{\top}\right]_{d} \\
& +24 \phi^{-3}\left[(\boldsymbol{P} \odot \boldsymbol{P}) \boldsymbol{G} \boldsymbol{Z} \boldsymbol{G}(\boldsymbol{P} \odot \boldsymbol{P})^{\top}\right]_{d} \\
& +8 \phi^{-3}\left[\{(\boldsymbol{P} \odot \boldsymbol{P} \odot \boldsymbol{P}) \boldsymbol{G} \mathbf{1}\}\left\{\boldsymbol{P} \boldsymbol{Z}_{d} \boldsymbol{G} \mathbf{1}\right\}^{\top}\right]_{d}, \\
& \mathbf{C}_{\mathbf{2} \beta}=-4 \phi^{-3}\left[\left(\boldsymbol{X}^{\top} \boldsymbol{W} \boldsymbol{X}\right)^{-1}\right]_{d}\left[\left\{\boldsymbol{P} \boldsymbol{Z}_{d} \boldsymbol{G} \mathbf{1}\right\}\left\{\boldsymbol{P} \boldsymbol{Z}_{d}(\boldsymbol{F}+2 \boldsymbol{G}) \mathbf{1}\right\}^{\top}\right]_{d} \\
& -8 \phi^{-3}\left[\left(\boldsymbol{X}^{\top} \boldsymbol{W} \boldsymbol{X}\right)^{-1}\right]_{d}\left[\boldsymbol{P G}(\boldsymbol{Z} \odot \boldsymbol{Z})(\boldsymbol{F}+2 \boldsymbol{G}) \boldsymbol{P}^{\top}\right]_{d} \\
& -8 \phi^{-3}\left[\left\{\boldsymbol{P} \boldsymbol{Z}_{d} \boldsymbol{G} \mathbf{1}\right\}\{(\boldsymbol{P} \odot \boldsymbol{P} \odot \boldsymbol{P})(\boldsymbol{F}+2 \boldsymbol{G}) \mathbf{1}\}^{\top}\right]_{d} \\
& -32 \phi^{-3}\left[(\boldsymbol{P} \odot \boldsymbol{P}) \boldsymbol{G} \boldsymbol{Z}(\boldsymbol{F}+2 \boldsymbol{G})(\boldsymbol{P} \odot \boldsymbol{P})^{\top}\right]_{d} \\
& -8 \phi^{-3}\left[\{(\boldsymbol{P} \odot \boldsymbol{P} \odot \boldsymbol{P}) \boldsymbol{G} \mathbf{1}\}\left\{\boldsymbol{P} \boldsymbol{Z}_{d}(\boldsymbol{F}+2 \boldsymbol{G}) \mathbf{1}\right\}^{\top}\right]_{d}, \\
& \mathbf{C}_{\mathbf{3} \beta}=\phi^{-3}\left[\left(\boldsymbol{X}^{\top} \boldsymbol{W} \boldsymbol{X}\right)^{-1}\right]_{d}\left[\left\{\boldsymbol{P} \boldsymbol{Z}_{d}(\boldsymbol{F}+2 \boldsymbol{G}) \mathbf{1}\right\}\left\{\boldsymbol{P} \boldsymbol{Z}_{d}(\boldsymbol{F}+2 \boldsymbol{G}) \mathbf{1}\right\}^{\top}\right]_{d} \\
& +2 \phi^{-3}\left\{\left[\boldsymbol{P} \boldsymbol{Z}_{d}(\boldsymbol{F}+2 \boldsymbol{G}) \mathbf{1}\right\}\{(\boldsymbol{P} \odot \boldsymbol{P} \odot \boldsymbol{P})(\boldsymbol{F}+2 \boldsymbol{G}) \mathbf{1}\}^{\top}\right]_{d} \\
& +2 \phi^{-3}\left[\left(\boldsymbol{X}^{\top} \boldsymbol{W} \boldsymbol{X}\right)^{-1}\right]_{d}\left[\boldsymbol{P}(\boldsymbol{F}+2 \boldsymbol{G})(\boldsymbol{Z} \odot \boldsymbol{Z})(\boldsymbol{F}+2 \boldsymbol{G}) \boldsymbol{P}^{\top}\right]_{d} \\
& +8 \phi^{-3}\left[(\boldsymbol{P} \odot \boldsymbol{P})(\boldsymbol{F}+2 \boldsymbol{G}) \boldsymbol{Z}(\boldsymbol{F}+2 \boldsymbol{G})(\boldsymbol{P} \odot \boldsymbol{P})^{\top}\right]_{d} \\
& +2 \phi^{-3}\left[\{(\boldsymbol{P} \odot \boldsymbol{P} \odot \boldsymbol{P})(\boldsymbol{F}+2 \boldsymbol{G}) \mathbf{1}\}\left\{\boldsymbol{P} \boldsymbol{Z}_{d}(\boldsymbol{F}+2 \boldsymbol{G}) \mathbf{1}\right\}^{\top}\right]_{d}, \\
& \mathbf{B}_{\mathbf{1} \beta}=\mathbf{C}_{\mathbf{1} \beta}+\mathbf{C}_{\mathbf{2} \beta}+\mathbf{C}_{\mathbf{3} \beta}, \\
& \mathbf{B}_{\mathbf{2} \beta}=\phi^{-3}\left[\left(\boldsymbol{X}^{\top} \boldsymbol{W} \boldsymbol{X}\right)^{-1}\right]_{d}\left[\left(\boldsymbol{P} \boldsymbol{Z}_{d} \boldsymbol{F} \mathbf{1}\right)\left(\boldsymbol{P} \boldsymbol{Z}_{d} \boldsymbol{F} \mathbf{1}\right)^{\top}\right]_{d} \\
& +2 \phi^{-3}\left[\left\{\boldsymbol{P} \boldsymbol{Z}_{d} \boldsymbol{F} \mathbf{1}\right\}\{(\boldsymbol{P} \odot \boldsymbol{P} \odot \boldsymbol{P}) \boldsymbol{F} \mathbf{1}\}^{\top}\right]_{d}, \\
& \mathbf{B}_{\mathbf{3} \beta}=\phi^{-3}\left[\left(\boldsymbol{X}^{\top} \boldsymbol{W} \boldsymbol{X}\right)^{-1}\right]_{d}\left[\left(\boldsymbol{P} \boldsymbol{Z}_{d} \boldsymbol{F} \mathbf{1}\right)\left(\boldsymbol{P} \boldsymbol{Z}_{d} \boldsymbol{F} \mathbf{1}\right)^{\top}\right]_{d}
\end{aligned}
$$




$$
\mathbf{A}_{22 \beta}=\mathbf{B}_{1 \beta}-2 \mathbf{B}_{2 \beta}+\mathbf{B}_{\mathbf{3} \beta} .
$$

\subsection{Resultados numéricos}

Apresentamos a seguir alguns resultados de simulações de Monte Carlo para comparar o coeficiente de curtose de ordem $n^{-1}$ com o coeficiente de curtose amostral, o qual é definido pela razão dos momentos amostrais. As simulações realizadas foram baseadas nos modelos de regressão gama e normal inversa com componentes sistemáticas definidas por

$$
\mu_{\ell}^{-1}=\beta_{1}+\beta_{2} x_{\ell 2} \quad \text { e } \log \left(\phi_{\ell}\right)=\lambda_{1}+\lambda_{2} s_{\ell 2},
$$

em que a covariável $x_{2}$ foi obtida a partir das distribuições $\mathcal{U}(0,1)$ e Weibull $(2,2)$ e consideramos $s_{2}=x_{2}$. Para cada tamanho da amostra as covariáveis foram mantidas constantes durante as simulações. Os valores verdadeiros dos parâmetros foram fixados em $\beta_{1}=3 / 2$, $\beta_{2}=1 / 2, \lambda_{1}=3$ e $\lambda_{2}=2$.

A quantidade de réplicas foi estabelecida em 10.000. Em cada réplica, ajustamos o modelo e calculamos o seu EMV $\hat{\boldsymbol{\theta}}$. O coeficiente de excesso de curtose amostral é definido por $\boldsymbol{g}_{2}=m_{4} / m_{2}^{2}-3$, em que $m_{r}=\sum_{i=1}^{10000}\left(\hat{\boldsymbol{\theta}}_{i}-\overline{\hat{\boldsymbol{\theta}}}\right)^{r} / 10000$ para $r=2$ e 4 e $\overline{\hat{\boldsymbol{\theta}}}=\sum_{i=1}^{10000} \hat{\boldsymbol{\theta}}_{i} / 10000$. Todas as simulações foram realizadas utilizando a linguagem de programação matricial ox (Doornik, 2006).

Podemos observar nas Tabelas 5.1 e 5.2 que, conforme esperado, o coeficiente de curtose de ordem $n^{-1}$ da distribuição do EMV de $\boldsymbol{\theta}$ decresce à medida que o tamanho da amostra aumenta, em concordância com a teoria assintótica de segunda ordem. Notamos também que a diferença entre os valores dos coeficientes de curtose de ordem $n^{-1}$ e amostral é maior quando o tamanho da amostra é pequeno. Percebemos ainda que os valores do coeficiente de curtose de ordem $n^{-1}$ são próximos, independentemente da distribuição da variável resposta. Finalmente, constatamos que a mudança na distribuição das covariáveis afeta, principalmente, os valores do coeficiente de curtose amostral.

Nas Figuras 5.1, 5.2, 5.3 e 5.4 comparamos as densidades estimadas dos EMVs com a densidade da distribuição normal. Os parâmetros utilizados para a distribuição normal são as médias e as variâncias obtidas dos respectivos EMVs. Podemos perceber em todas as Figuras que a aproximação das distribuições de $\hat{\boldsymbol{\lambda}}$ pela distribuição normal não é apropriada. 
Tabela 5.1: Coeficiente de excesso de curtose amostral e de ordem $n^{-1}$ e covariável $\mathcal{U}(0,1)$.

\begin{tabular}{|c|c|c|c|c|c|c|c|c|c|}
\hline & \multirow[b]{2}{*}{$n$} & \multicolumn{4}{|c|}{ modelo gama } & \multicolumn{4}{|c|}{ modelo normal inversa } \\
\hline & & $\hat{\beta}_{1}$ & $\hat{\beta}_{2}$ & $\hat{\lambda}_{1}$ & $\hat{\lambda}_{2}$ & $\hat{\beta}_{1}$ & $\hat{\beta}_{2}$ & $\hat{\lambda}_{1}$ & $\hat{\lambda}_{2}$ \\
\hline$g_{2}$ & 10 & 1,0880 & 0,8318 & 3,1606 & 2,5669 & 1,3535 & 1,5003 & 3,8364 & 3,7986 \\
\hline$\gamma_{2}$ & 10 & 0,7762 & 0,7669 & 2,0275 & 1,6132 & 0,8346 & 0,8043 & 2,2072 & 1,8747 \\
\hline$g_{2}$ & & 0,2650 & 0,2131 & 0,7189 & 0,6098 & 0,3468 & 0,2595 & 0,7722 & 0,6014 \\
\hline$\gamma_{2}$ & 20 & 0,1779 & 0,1858 & 0,4585 & 0,4022 & 0,1892 & 0,1911 & 0,5008 & 0,4036 \\
\hline$g_{2}$ & 40 & 0,0619 & 0,0954 & 0,0591 & 0,1678 & 0,0595 & 0,0756 & 0,3070 & 0,3074 \\
\hline$\gamma_{2}$ & 40 & 0,0414 & 0,0472 & 0,1012 & 0,1012 & 0,0446 & 0,0474 & 0,1182 & 0,1095 \\
\hline$g_{2}$ & 60 & 0,0379 & 0,0124 & 0,0585 & 0,0590 & 0,0511 & 0,0635 & 0,0454 & 0,0900 \\
\hline$\gamma_{2}$ & & 0,0187 & 0,0213 & 0,0474 & 0,0487 & 0,0200 & 0,0211 & 0,0533 & 0,0493 \\
\hline
\end{tabular}

Tabela 5.2: Coeficiente de excesso de curtose amostral e de ordem $n^{-1}$ e covariável Weibull(2,2).

\begin{tabular}{|c|c|c|c|c|c|c|c|c|c|}
\hline & \multirow[b]{2}{*}{$n$} & \multicolumn{4}{|c|}{ modelo gama } & \multicolumn{4}{|c|}{ modelo normal inversa } \\
\hline & & $\hat{\beta}_{1}$ & $\hat{\beta}_{2}$ & $\hat{\lambda}_{1}$ & $\hat{\lambda}_{2}$ & $\hat{\beta}_{1}$ & $\hat{\beta}_{2}$ & $\hat{\lambda}_{1}$ & $\hat{\lambda}_{2}$ \\
\hline$g_{2}$ & 10 & 1,6738 & 2,1371 & 2,3880 & 2,0480 & 1,1027 & 1,2252 & 3,5583 & 3,3991 \\
\hline$\gamma_{2}$ & 10 & 0,7423 & 0,7875 & 1,7224 & 1,7187 & 0,8194 & 0,8057 & 2,1066 & 1,9131 \\
\hline$g_{2}$ & & 0,1357 & 0,1905 & 0,6887 & 0,8034 & 0,3366 & 0,2726 & 0,8205 & 0,6674 \\
\hline$\gamma_{2}$ & 20 & 0,1777 & 0,2003 & 0,4123 & 0,4612 & 0,1890 & 0,1903 & 0,4957 & 0,4246 \\
\hline$g_{2}$ & 40 & 0,0273 & 0,0078 & 0,2147 & 0,3240 & 0,0156 & 0,1401 & 0,3923 & 0,5393 \\
\hline$\gamma_{2}$ & 40 & 0,0491 & 0,0683 & 0,1009 & 0,1660 & 0,0525 & 0,0717 & 0,1091 & 0,1781 \\
\hline$g_{2}$ & 60 & 0,0128 & 0,0898 & 0,1938 & 0,3147 & 0,0693 & 0,1162 & 0,0969 & 0,2445 \\
\hline$\gamma_{2}$ & 00 & 0,0223 & 0,0309 & 0,0492 & 0,0790 & 0,0250 & 0,0339 & 0,0517 & 0,0827 \\
\hline
\end{tabular}



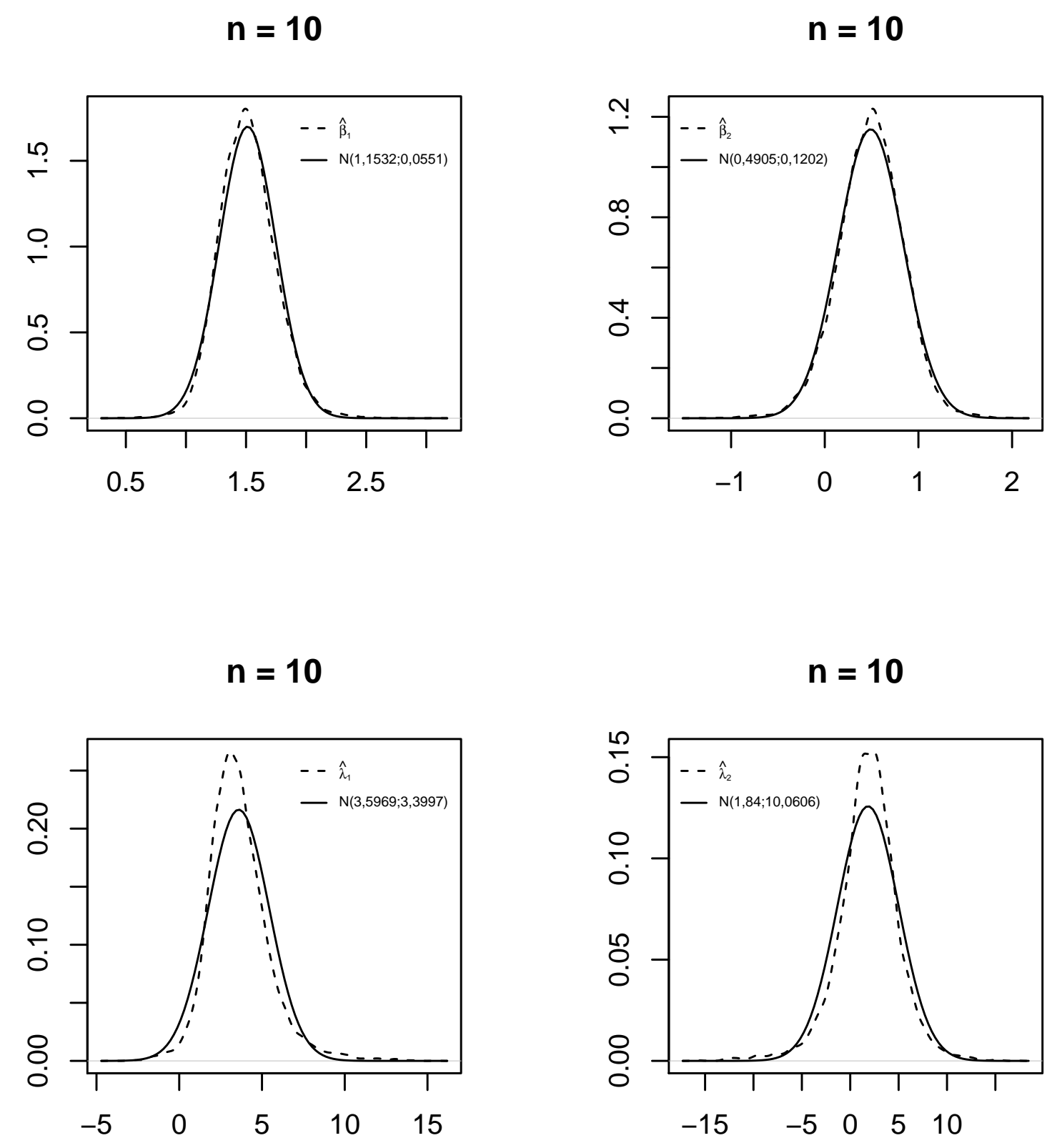

Figura 5.1: Densidades estimada e normal; modelo gama e covariável $\mathcal{U}(0,1)$. 

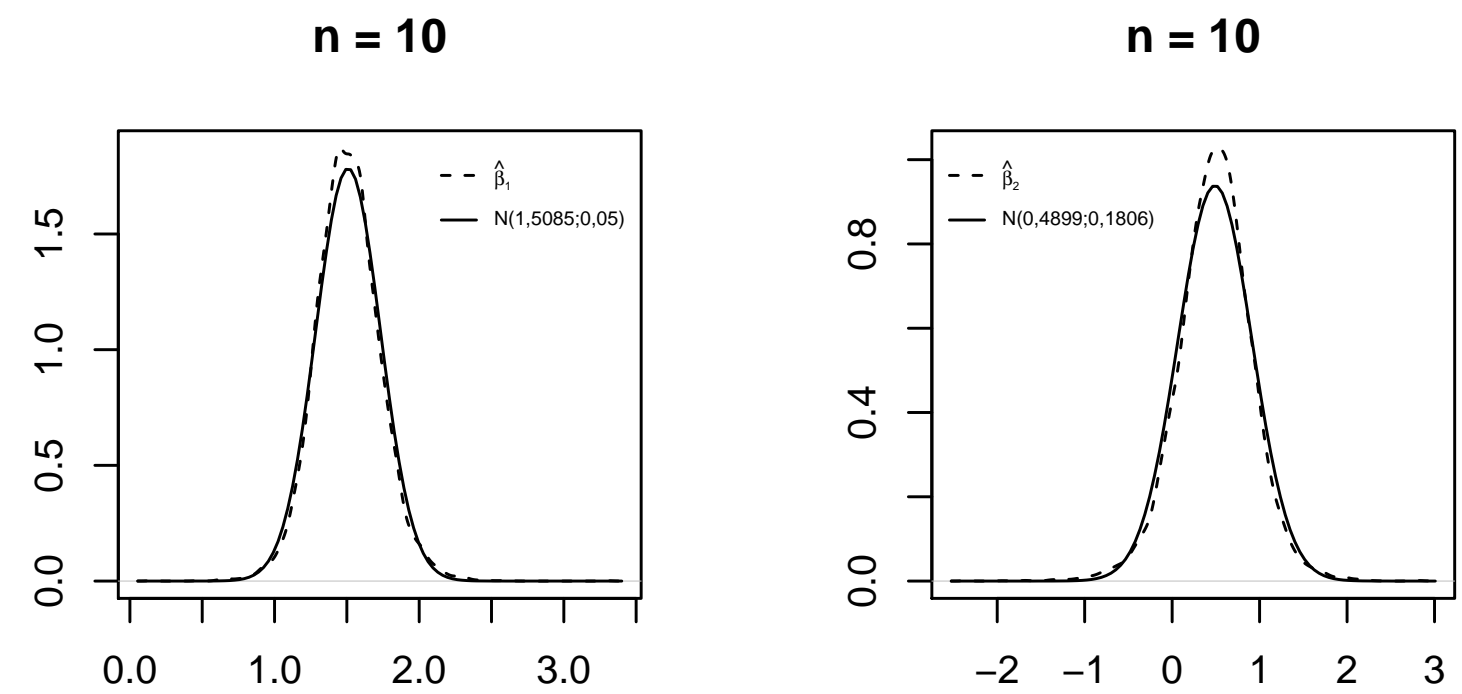

$$
n=10
$$
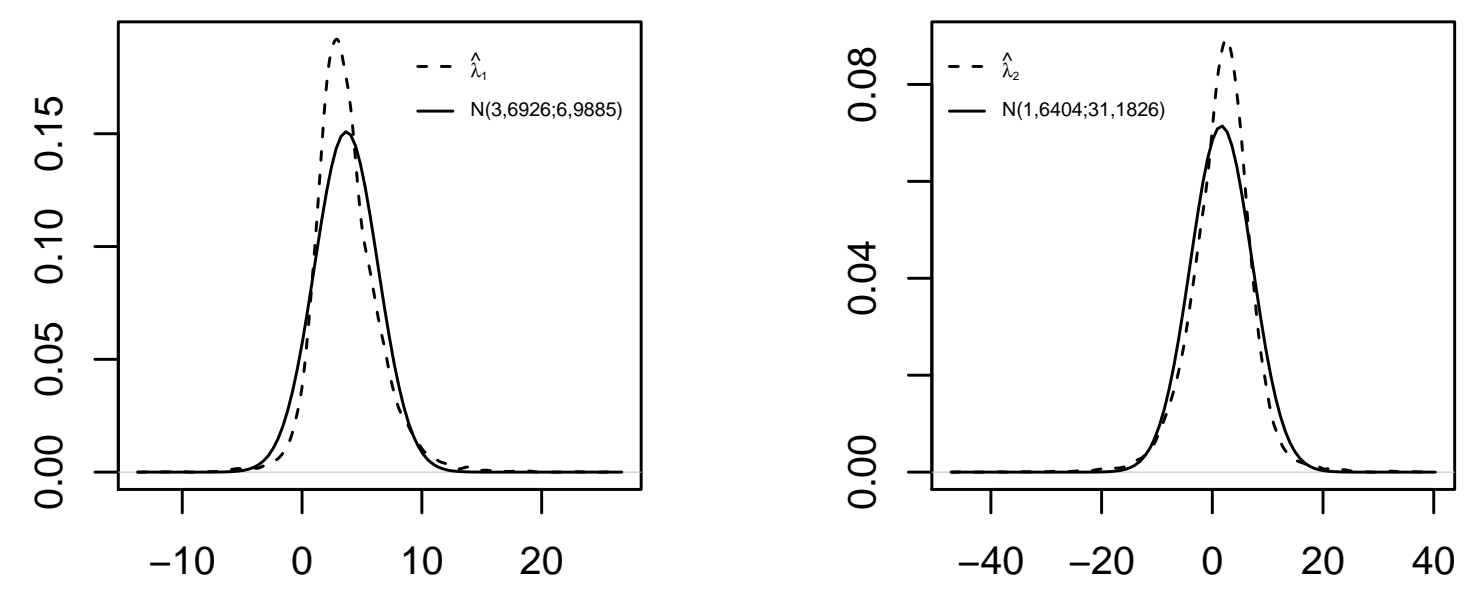

Figura 5.2: Densidades estimada e normal; modelo normal inversa e covariável $\mathcal{U}(0,1)$. 

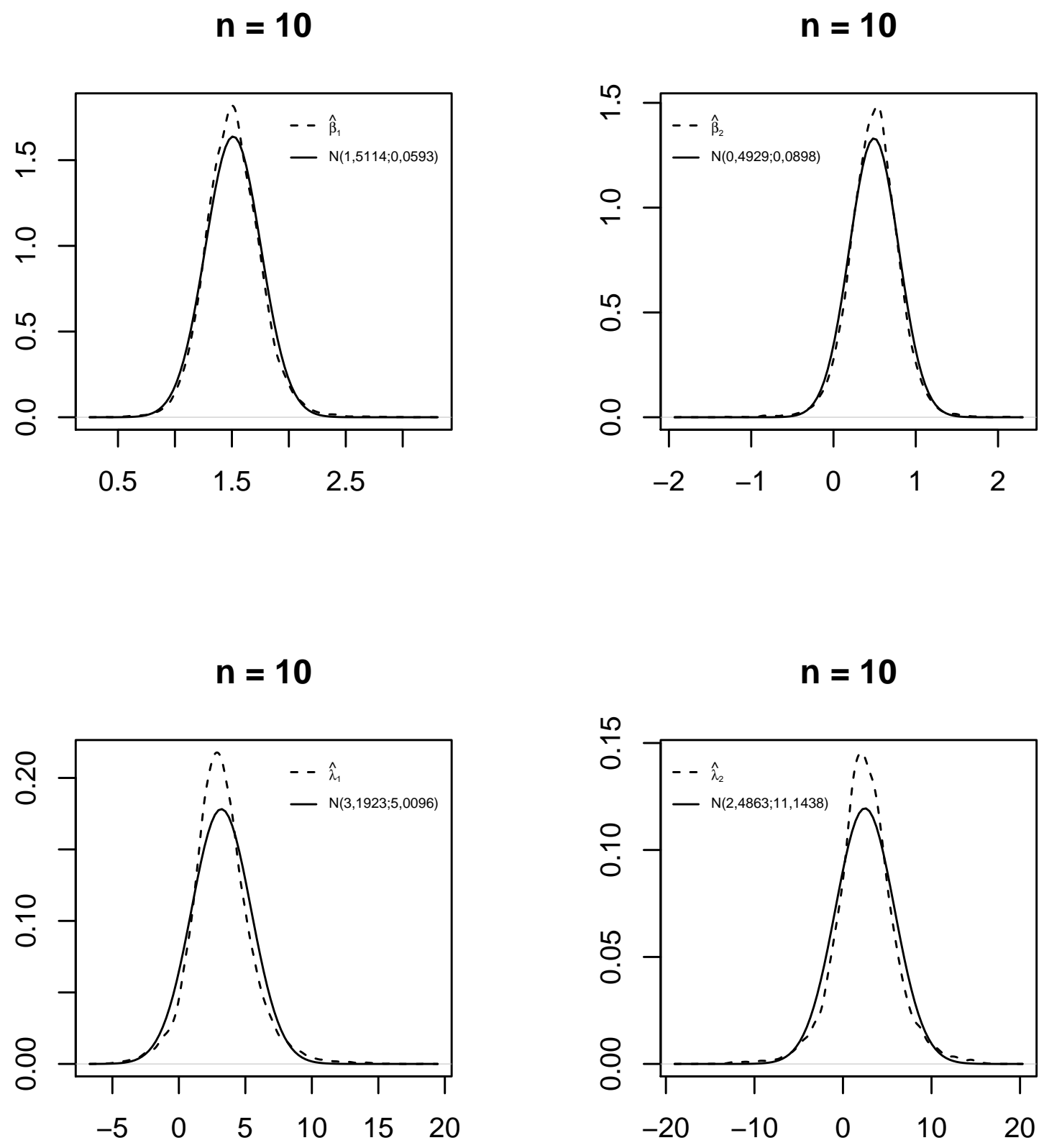

Figura 5.3: Densidades estimada e normal; modelo gama e covariável Weibull(2,2). 
$n=10$

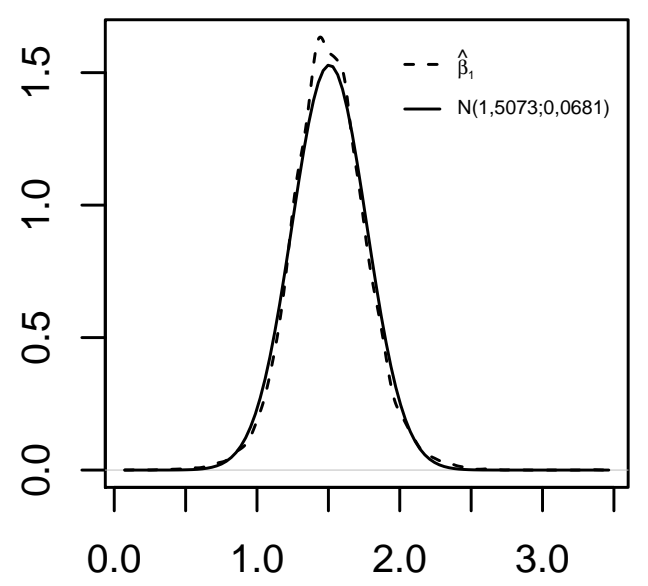

$\mathrm{n}=\mathbf{1 0}$

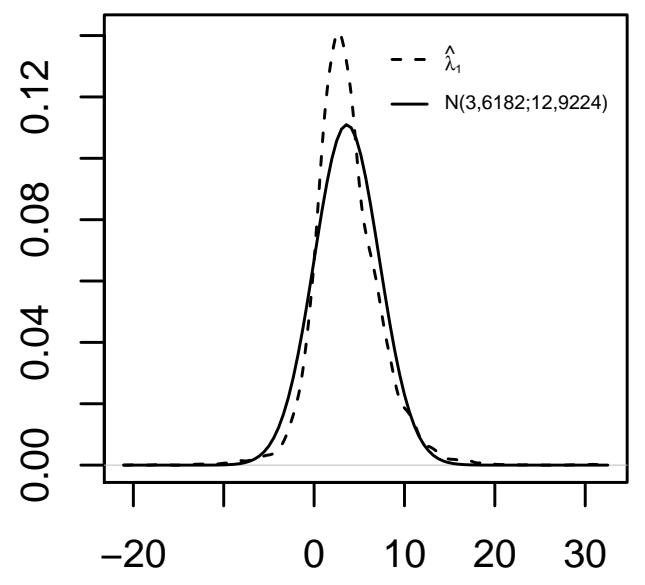

$n=10$

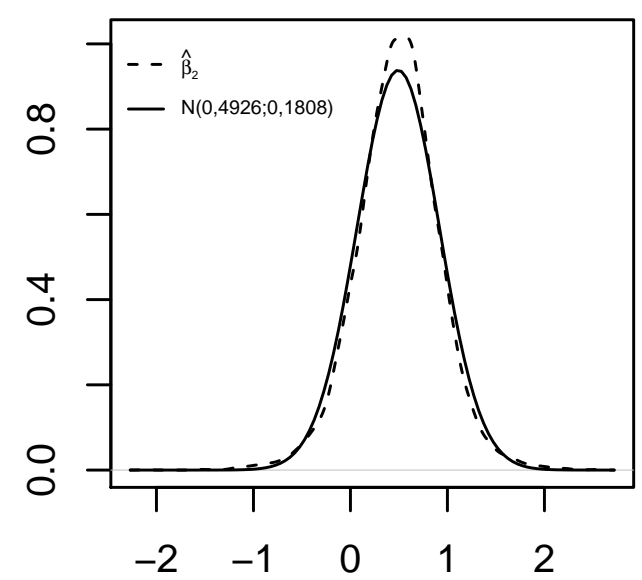

$n=10$

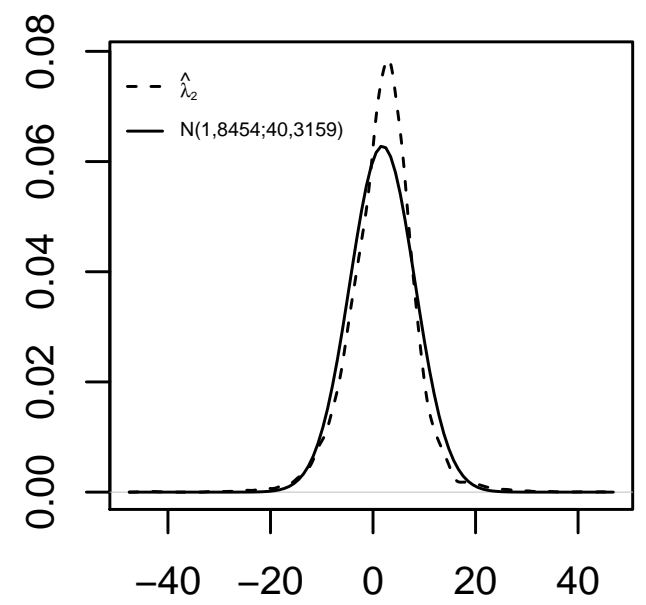

Figura 5.4: Densidades estimada e normal; modelo normal inversa e covariável Weibull(2,2). 


\subsection{Aplicação}

Consideremos os dados descritos em Montgomery et al. (2001, p. 76) referentes a serviços de manutenção e reposição de latas e garrafas de bebidas em máquinas de venda automáticas. A variável resposta $Y$ é o tempo (em minutos) gasto no serviço, as covariáveis $x_{2}$ e $x_{3}$ utilizadas são, respectivamente, o número de bebidas estocadas e a distância percorrida (em pés). Para modelar esses dados Lemonte (2013) assumiu um modelo de regressão gama heteroscedástico $G\left(\mu_{\ell}, \phi_{\ell}\right)$, uma vez que a variável resposta é positiva. As componentes sistemáticas do modelo foram definidas como $\log \left(\mu_{\ell}\right)=\beta_{1}+\beta_{2} x_{\ell 2}+\beta_{3} x_{\ell 3}$ e $\log \left(\phi_{\ell}\right)=\lambda_{1}+\lambda_{2} x_{\ell 2}$ para $\ell=1, \ldots, 25$.

Exibimos na Tabela 5.3 as estimativas dos parâmetros, bem como seus coeficientes de assimetria e curtose estimados. Podemos observar nesta tabela que a curtose estimada para a distribuição dos EMVs sugere que a aproximação normal não é adequada, embora as distribuições dos EMVs dos parâmetros $\beta_{1}, \beta_{2}$ e $\beta_{3}$ aparentem ser simétricas.

Tabela 5.3: Estimativas dos parâmetros, assimetria e curtose estimada.

\begin{tabular}{ccccc}
\hline Parâmetro & Estimativa & Erro padrão & $\gamma_{1}$ & $\gamma_{2}$ \\
\hline$\beta_{1}$ & 2,4231 & 0,0486 & $-0,0307$ & 0,1537 \\
$\beta_{2}$ & 0,0465 & 0,0055 & $-0,0076$ & 0,4264 \\
$\beta_{3}$ & 0,0004 & 0,0001 & 0,0084 & 0,4877 \\
$\lambda_{1}$ & 2,7580 & 0,4615 & 0,1307 & 0,2832 \\
$\lambda_{2}$ & 0,1168 & 0,0419 & 0,5275 & 0,9876 \\
\hline
\end{tabular}

\subsection{Conclusões}

Neste capítulo, consideramos a classe dos MLGHs e obtivemos fórmulas matriciais para o coeficiente de curtose de ordem $n^{-1}$. Estas fórmulas são úteis para verificar se a aproximação da distribuição do EMV em relação à distribuição normal é adequada em amostras de tamanho pequeno a moderado. Os estudos de simulação indicaram que o coeficiente de curtose amostral é mais sensível à mudança na distribuição das covariáveis, enquanto que o coeficiente de curtose de ordem $n^{-1}$, de um modo geral, independe da distribuição da variável resposta e da distribuição das covariáveis. 


\section{Capítulo 6}

\section{Conclusões e Pesquisas Futuras}

Nesta tese, obtivemos refinamentos assintóticos em modelos lineares generalizados heteroscedásticos.

No Capítulo 2, derivamos a matriz de covariâncias de ordem $n^{-2}$ dos EMVs corrigidos pelo viés de ordem $n^{-1}$, que pode ser utilizada para modificar o teste de Wald. Os estudos de simulação indicaram que os valores da matriz de covariâncias de segunda ordem dos EMVs corrigidos encontram-se mais próximos dos valores da matriz de covariâncias amostrais quando comparados com os da matriz de covariâncias de primeira ordem dos EMVs corrigidos. Estes estudos mostraram também que o uso da matriz de covariâncias de segunda ordem juntamente com os EMVs corrigidos produziram uma melhora significativa em relação às distorções de tamanho do teste $W_{2}$ quando comparado com os testes $W$ e $W_{1}$. Os estudos mostraram ainda que o teste $W_{\text {boot }}$ apresentou um desempenho melhor que os demais testes.

No Capítulo 3, calculamos o fator de correção tipo-Bartlett para a estatística gradiente. Os estudos de simulação mostraram que os testes da razão de verossimilhanças, Wald, Wald modificado $\left(W_{2}\right)$ e gradiente podem ser severamente liberais em amostras de tamanho pequeno, rejeitando a hipótese nula muito mais do que o esperado para cada nível nominal selecionado. O teste escore mostrou-se menos distorcido, podendo ser conservativo em alguns casos. O fator de correção tipo-Bartlett para os testes escore e gradiente fez com que a taxa de rejeição empírica ficasse mais próxima do nível nominal do teste. Os testes da razão de verossimilhanças ajustado e corrigido apresentaram maior distorção de tamanho que os testes escore e gradiente corrigidos. Os testes bootstrap conduziram a uma considerável redução da distorção de tamanho observada para os testes assintóticos, principalmente para os testes da razão de verossimilhanças e Wald, cujas versões usuais são tipicamente liberais.

No Capítulo 4, deduzimos fórmulas bastante simples para o cálculo do coeficiente de assimetria de ordem $n^{-1 / 2}$, o qual pode servir como uma medida de afastamento da distribuição normal. Os estudos de simulação indicaram que, em geral, o coeficiente de assimetria da distribuição dos EMVs é influenciado tanto pela distribuição da variável resposta quanto pela distribuição da covariável.

No Capítulo 5, desenvolvemos fórmulas matriciais para o coeficiente de curtose de ordem 
$n^{-1}$, o qual pode ser útil para verificar se a aproximação da distribuição do EMV em relação à distribuição normal é adequada em amostras de tamanho pequeno a moderado. Os estudos de simulação indicaram que, de um modo geral, o coeficiente de curtose de ordem $n^{-1}$ independe da distribuição da variável resposta e da distribuição das covariáveis, enquanto que o coeficiente de curtose amostral é mais sensível à mudança na distribuição das covariáveis.

Diversos trabalhos poderão ser desenvolvidos a partir das ideias desta tese. Podemos citar:

1. Cálculo da matriz de covariâncias de ordem $n^{-2}$ dos estimadores corrigidos pelo viés de ordem $n^{-1}$ para os modelos de regressão valor extremo;

2. Obtenção dos fatores de correção tipo-Bartlett para a estatística gradiente em modelos de dispersão heteroscedásticos;

3. Cálculo do coeficiente de curtose para os modelos não-lineares da família exponencial;

4. Implementação das quantidades obtidas nesta tese no software $\mathrm{R}$ ( $\mathrm{R}$ Development Core, 2009).

Desejamos, através dos resultados desta tese, ter colaborado de forma expressiva na pesquisa de refinamentos de métodos assintóticos na classe dos modelos lineares generalizados heteroscedásticos. 


\section{Apêndice A}

\section{Identidades de Bartlett e Cumulantes}

\section{A.1 Identidades de Bartlett}

As identidades abaixo podem ser encontradas em Cordeiro (1991, p. 120).

- $\kappa_{r}=0$,

- $\kappa_{r s}+\kappa_{r, s}=0$,

- $\kappa_{r s t}+\kappa_{r, s t}-\kappa_{s t}^{(r)}=0$,

- $\kappa_{r, s, t}-2 \kappa_{r s t}+\Sigma_{(3)} \kappa_{r s}^{(t)}=0$,

- $\kappa_{r s t u}+\kappa_{r, s t u}-\kappa_{s t u}^{(r)}=0$,

- $\kappa_{r, s, t u}=\kappa_{r s t u}-\kappa_{r t u}^{(s)}-\kappa_{s t u}^{(r)}+\kappa_{t u}^{(r s)}-\kappa_{r s, t u}$,

- $\kappa_{r, s, t, u}=-3 \kappa_{r s t u}+2 \Sigma_{(4)} \kappa_{r s t}^{(u)}-\Sigma_{(6)} \kappa_{r s}^{(t u)}+\Sigma_{(3)} \kappa_{r s, t u}$,

- $\kappa_{r s t u}+\Sigma_{(4)} \kappa_{r, s t u}+\Sigma_{(3)} \kappa_{r s, t u}+\Sigma_{(6)} \kappa_{r, s, t u}+\kappa_{r, s, t, u}=0$,

em que $\Sigma_{(k)}$ representa o somatório sobre todas as k combinações de índices.

\section{A.2 Cumulantes}

- $\kappa_{r s}=-\sum_{\ell=1}^{n} \phi_{\ell} w_{\ell} x_{\ell r} x_{\ell s}$,

- $\kappa_{R S}=\sum_{\ell=1}^{n} d_{2 \ell} \phi_{1 \ell}^{2} s_{\ell R} s_{\ell S}$,

- $\kappa_{r s t}=-\sum_{\ell=1}^{n} \phi_{\ell}\left(f_{\ell}+2 g_{\ell}\right) x_{\ell r} x_{\ell s} x_{\ell t}$,

- $\kappa_{r s, t}=\sum_{\ell=1}^{n} \phi_{\ell} g_{\ell} x_{\ell r} x_{\ell s} x_{\ell t}$,

- $\kappa_{r, s, t}=\sum_{\ell=1}^{n} \phi_{\ell}\left(f_{\ell}-g_{\ell}\right) x_{\ell r} x_{\ell s} x_{\ell t}$, 
- $\kappa_{R s, t}=-\kappa_{R s t}=-\kappa_{R, s, t}=-\kappa_{s t}^{(R)}=\sum_{\ell=1}^{n} \phi_{1 \ell} w_{\ell} x_{\ell s} x_{\ell t} s_{\ell R}$,

- $\kappa_{R, s t}=\kappa_{R S t}=\kappa_{R, S, t}=\kappa_{R, S t}=\kappa_{R S, t}=\kappa_{R S}^{(t)}=\kappa_{R s}^{(t)}=\kappa_{R s}^{(T)}=0$,

- $\kappa_{R S T}=\sum_{\ell=1}^{n}\left(d_{3 \ell} \phi_{1 \ell}^{3}+3 d_{2 \ell} \phi_{1 \ell} \phi_{2 \ell}\right) s_{\ell R} s_{\ell S} s_{\ell T}$,

- $\kappa_{R S, T}=-\sum_{\ell=1}^{n} d_{2 \ell} \phi_{1 \ell} \phi_{2 \ell} s_{\ell R} s_{\ell S} s_{\ell T}$,

- $\kappa_{R, S, T}=-\sum_{\ell=1}^{n} d_{3 \ell} \phi_{1 \ell}^{3} s_{\ell R} s_{\ell S} s_{\ell T}$,

- $\kappa_{R S}^{(T)}=\sum_{\ell=1}^{n}\left(d_{3 \ell} \phi_{1 \ell}^{3}+2 d_{2 \ell} \phi_{1 \ell} \phi_{2 \ell}\right) s_{\ell R} s_{\ell S} s_{\ell T}$,

$$
\begin{aligned}
-\kappa_{r s t u}=\sum_{\ell=1}^{n} \phi_{\ell}\{ & -\frac{6}{V^{3}}\left(\frac{d V}{d \mu}\right)^{2}\left(\frac{d \mu}{d \eta}\right)^{4}+\frac{3}{V^{2}} \frac{d^{2} V}{d \mu^{2}}\left(\frac{d \mu}{d \eta}\right)^{4}+\frac{12}{V^{2}} \frac{d V}{d \mu}\left(\frac{d \mu}{d \eta}\right)^{2} \frac{d^{2} \mu}{d \eta^{2}}-\frac{3}{V}\left(\frac{d^{2} \mu}{d \eta^{2}}\right)^{2} \\
& \left.-\frac{4}{V} \frac{d \mu}{d \eta} \frac{d^{3} \mu}{d \eta^{3}}\right\}_{\ell}^{x_{\ell r} x_{\ell s} x_{\ell t} x_{\ell u}},
\end{aligned}
$$

- $\kappa_{r s, t u}=\sum_{\ell=1}^{n} \phi_{\ell}\left\{\frac{1}{V^{3}}\left(\frac{d V}{d \mu}\right)^{2}\left(\frac{d \mu}{d \eta}\right)^{4}-\frac{2}{V^{2}} \frac{d V}{d \mu}\left(\frac{d \mu}{d \eta}\right)^{2} \frac{d^{2} \mu}{d \eta^{2}}+\frac{1}{V}\left(\frac{d^{2} \mu}{d \eta^{2}}\right)^{2}\right\}_{\ell} x_{\ell r} x_{\ell s} x_{\ell t} x_{\ell u}$

- $\kappa_{r, s, t u}=\sum_{\ell=1}^{n} \phi_{\ell}\left\{\frac{1}{V^{2}} \frac{d V}{d \mu}\left(\frac{d \mu}{d \eta}\right)^{2} \frac{d^{2} \mu}{d \eta^{2}}-\frac{1}{V^{3}}\left(\frac{d V}{d \mu}\right)^{2}\left(\frac{d \mu}{d \eta}\right)^{4}\right\}_{\ell} x_{\ell r} x_{\ell s} x_{\ell t} x_{\ell u}$

- $\kappa_{r, s, t, u}=\sum_{\ell=1}^{n} \phi_{\ell}\left\{\frac{1}{V^{3}}\left(\frac{d V}{d \mu}\right)^{2}\left(\frac{d \mu}{d \eta}\right)^{4}+\frac{1}{V^{2}} \frac{d^{2} V}{d \mu^{2}}\left(\frac{d \mu}{d \eta}\right)^{4}\right\}_{\ell} x_{\ell r} x_{\ell s} x_{\ell t} x_{\ell u}$

- $\kappa_{r s t, u}=\sum_{\ell=1}^{n} \phi_{\ell}\left\{\frac{2}{V^{3}}\left(\frac{d V}{d \mu}\right)^{2}\left(\frac{d \mu}{d \eta}\right)^{4}-\frac{1}{V^{2}} \frac{d^{2} V}{d \mu^{2}}\left(\frac{d \mu}{d \eta}\right)^{4}-\frac{3}{V^{2}} \frac{d V}{d \mu}\left(\frac{d \mu}{d \eta}\right)^{2} \frac{d^{2} \mu}{d \eta^{2}}+\frac{1}{V} \frac{d \mu}{d \eta} \frac{d^{3} \mu}{d \eta^{3}}\right\}_{\ell}$

$$
x_{\ell r} x_{\ell s} x_{\ell t} x_{\ell u},
$$

- $\kappa_{\text {stu }}^{(r)}=\sum_{\ell=1}^{n} \phi_{\ell}\left\{-\frac{4}{V^{3}}\left(\frac{d V}{d \mu}\right)^{2}\left(\frac{d \mu}{d \eta}\right)^{4}+\frac{2}{V^{2}} \frac{d^{2} V}{d \mu^{2}}\left(\frac{d \mu}{d \eta}\right)^{4}+\frac{9}{V^{2}} \frac{d V}{d \mu}\left(\frac{d \mu}{d \eta}\right)^{2} \frac{d^{2} \mu}{d \eta^{2}}-\frac{3}{V}\left(\frac{d^{2} \mu}{d \eta^{2}}\right)^{2}\right.$

$$
\left.-\frac{3}{V} \frac{d \mu}{d \eta} \frac{d^{3} \mu}{d \eta^{3}}\right\}_{\ell} x_{\ell r} x_{\ell s} x_{\ell t} x_{\ell u},
$$


- $\kappa_{t u}^{(r s)}=\sum_{\ell=1}^{n} \phi_{\ell}\left\{-\frac{2}{V^{3}}\left(\frac{d V}{d \mu}\right)^{2}\left(\frac{d \mu}{d \eta}\right)^{4}+\frac{1}{V^{2}} \frac{d^{2} V}{d \mu^{2}}\left(\frac{d \mu}{d \eta}\right)^{4}+\frac{5}{V^{2}} \frac{d V}{d \mu}\left(\frac{d \mu}{d \eta}\right)^{2} \frac{d^{2} \mu}{d \eta^{2}}-\frac{2}{V}\left(\frac{d^{2} \mu}{d \eta^{2}}\right)^{2}\right.$

$$
\left.-\frac{2}{V} \frac{d \mu}{d \eta} \frac{d^{3} \mu}{d \eta^{3}}\right\}_{\ell} x_{\ell r} x_{\ell s} x_{\ell t} x_{\ell u}
$$

- $\kappa_{r s t U}=-\sum_{\ell=1}^{n} \phi_{1 \ell}\left(f_{\ell}+2 g_{\ell}\right) x_{\ell r} x_{\ell s} x_{\ell t} s_{\ell U}$,

- $\kappa_{r, s t U}=-\kappa_{r s, t, U}=\kappa_{r s, t U}=\sum_{\ell=1}^{n} \phi_{1 \ell} g_{\ell} x_{\ell r} x_{\ell s} x_{\ell t} s_{\ell U}$,

- $\kappa_{r, s, t U}=\sum_{\ell=1}^{n} \phi_{1 \ell}\left(f_{\ell}-g_{\ell}\right) x_{\ell r} x_{\ell s} x_{\ell t} s_{\ell U}$,

- $\kappa_{r s t, U}=0$,

- $\kappa_{R S t u}=\kappa_{R S, t, u}=\kappa_{S t u}^{(R)}=-\kappa_{R S t, u}=-\sum_{\ell=1}^{n} \phi_{2 \ell} w_{\ell} x_{\ell t} x_{\ell u} s_{\ell R} s_{\ell S}$,

- $\kappa_{R t, S u}=-\kappa_{R, S t, u}=\frac{1}{2} \kappa_{R, S, t, u}=\sum_{\ell=1}^{n} \phi_{1 \ell}^{2} \phi_{\ell}^{-1} w_{\ell} x_{\ell t} x_{\ell u} s_{\ell R} s_{\ell S}$,

- $\kappa_{R, S t u}=\kappa_{R, S, t u}=\kappa_{R S, t u}=\kappa_{R S t}^{(u)}=0$,

- $\kappa_{R S T U}=\sum_{\ell=1}^{n}\left(d_{4 \ell} \phi_{1 \ell}^{4}+6 d_{3 \ell} \phi_{1 \ell}^{2} \phi_{2 \ell}+3 d_{2 \ell} \phi_{2 \ell}^{2}+4 d_{2 \ell} \phi_{1 \ell} \phi_{3 \ell}\right) s_{\ell R} s_{\ell S} s_{\ell T} s_{\ell U}$,

- $\kappa_{R, S, T U}=-\sum_{\ell=1}^{n} d_{3 \ell} \phi_{1 \ell}^{2} \phi_{2 \ell} s_{\ell R} s_{\ell S} s_{\ell T} s_{\ell U}$,

- $\kappa_{R S, T U}=-\sum_{\ell=1}^{n} d_{2 \ell} \phi_{2 \ell}^{2} s_{\ell R} s_{\ell S} s_{\ell T} s_{\ell U}$,

- $\kappa_{R, S, T, U}=-\sum_{\ell=1}^{n} d_{4 \ell} \phi_{1 \ell}^{4} s_{\ell R} s_{\ell S} s_{\ell T} s_{\ell U}$,

- $\kappa_{R S}^{(T U)}=\sum_{\ell=1}^{n}\left(d_{4 \ell} \phi_{1 \ell}^{4}+5 d_{3 \ell} \phi_{1 \ell}^{2} \phi_{2 \ell}+2 d_{2 \ell} \phi_{2 \ell}^{2}+2 d_{2 \ell} \phi_{1 \ell} \phi_{3 \ell}\right) s_{\ell R} s_{\ell S} s_{\ell T} s_{\ell U}$,

- $\kappa_{R S T}^{(U)}=\sum_{\ell=1}^{n}\left(d_{4 \ell} \phi_{1 \ell}^{4}+6 d_{3 \ell} \phi_{1 \ell}^{2} \phi_{2 \ell}+3 d_{2 \ell} \phi_{2 \ell}^{2}+3 d_{2 \ell} \phi_{1 \ell} \phi_{3 \ell}\right) s_{\ell R} s_{\ell S} s_{\ell T} s_{\ell U}$. 


\section{Apêndice B}

\section{Fator de correção tipo-Bartlett}

Neste apêndice apresentamos a obtenção das quantidades $A_{1}, A_{2}$ e $A_{3}$ referentes ao teste de hipóteses $\mathcal{H}_{0}: \boldsymbol{\beta}_{1}=\boldsymbol{\beta}_{1}^{(0)}, \boldsymbol{\lambda}_{1}=\boldsymbol{\lambda}_{1}^{(0)}$ versus $\mathcal{H}_{1}$ : pelo menos umas das igualdades da hipótese $\mathcal{H}_{0}$ não se verifica.

Seja $\boldsymbol{\theta}=\left(\boldsymbol{\beta}^{\top}, \boldsymbol{\lambda}^{\top}\right)^{\top}$, devido à ortogonalidade global entre $\boldsymbol{\beta}$ e $\boldsymbol{\lambda}$, a matriz de informação total de Fisher $\boldsymbol{K}(\boldsymbol{\theta})$ e sua inversa $\boldsymbol{K}^{-1}(\boldsymbol{\theta})$ são bloco-diagonais e podem ser escritas como

$$
\boldsymbol{K}(\boldsymbol{\theta})=\left[\begin{array}{cc}
\boldsymbol{K}_{\beta} & \mathbf{0} \\
\mathbf{0} & \boldsymbol{K}_{\lambda}
\end{array}\right] \quad \text { e } \quad \boldsymbol{K}^{-1}(\boldsymbol{\theta})=\left[\begin{array}{cc}
\boldsymbol{K}_{\beta}^{-1} & \mathbf{0} \\
\mathbf{0} & \boldsymbol{K}_{\lambda}^{-1}
\end{array}\right]
$$

A partição $\boldsymbol{\beta}=\left(\boldsymbol{\beta}_{1}^{\top}, \boldsymbol{\beta}_{2}^{\top}\right)^{\top}$, com $\boldsymbol{\beta}_{1}=\left(\beta_{1}, \ldots, \beta_{p_{1}}\right)^{\top}$ e $\boldsymbol{\beta}_{2}=\left(\beta_{p_{1}+1}, \ldots, \beta_{p}\right)^{\top}$, e $\boldsymbol{\lambda}=$ $\left(\boldsymbol{\lambda}_{1}^{\top}, \boldsymbol{\lambda}_{2}^{\top}\right)^{\top} \operatorname{com} \boldsymbol{\lambda}_{1}=\left(\lambda_{1}, \ldots, \lambda_{q_{1}}\right)^{\top}$ e $\boldsymbol{\lambda}_{2}=\left(\lambda_{q_{1}+1}, \ldots, \lambda_{q}\right)^{\top}$ induz às seguintes partições

$$
\begin{gathered}
\boldsymbol{K}_{\beta}=\left[\begin{array}{ll}
\boldsymbol{K}_{\beta 11} & \boldsymbol{K}_{\beta 12} \\
\boldsymbol{K}_{\beta 21} & \boldsymbol{K}_{\beta 22}
\end{array}\right], \quad \boldsymbol{K}_{\beta}^{-1}=\left[\begin{array}{ll}
\boldsymbol{K}_{\beta}^{11} & \boldsymbol{K}_{\beta}^{12} \\
\boldsymbol{K}_{\beta}^{21} & \boldsymbol{K}_{\beta}^{22}
\end{array}\right], \\
\boldsymbol{K}_{\lambda}=\left[\begin{array}{ll}
\boldsymbol{K}_{\lambda 11} & \boldsymbol{K}_{\lambda 12} \\
\boldsymbol{K}_{\lambda 21} & \boldsymbol{K}_{\lambda 22}
\end{array}\right] \quad \text { e } \quad \boldsymbol{K}_{\lambda}^{-1}=\left[\begin{array}{ll}
\boldsymbol{K}_{\lambda}^{11} & \boldsymbol{K}_{\lambda}^{12} \\
\boldsymbol{K}_{\lambda}^{21} & \boldsymbol{K}_{\lambda}^{22}
\end{array}\right] .
\end{gathered}
$$

Sejam as matrizes

$$
\boldsymbol{A}=\left[\begin{array}{cc}
\boldsymbol{A}_{\beta} & \mathbf{0} \\
\mathbf{0} & \boldsymbol{A}_{\lambda}
\end{array}\right] \quad \text { e } \boldsymbol{M}=\left[\begin{array}{cc}
\boldsymbol{M}_{\beta} & \mathbf{0} \\
\mathbf{0} & \boldsymbol{M}_{\lambda}
\end{array}\right]
$$

em que

$$
\boldsymbol{A}_{\beta}=\left[\begin{array}{cc}
\mathbf{0} & \mathbf{0} \\
\mathbf{0} & \boldsymbol{K}_{\beta 22}^{-1}
\end{array}\right], \quad \boldsymbol{A}_{\lambda}=\left[\begin{array}{cc}
\mathbf{0} & \mathbf{0} \\
\mathbf{0} & \boldsymbol{K}_{\lambda 22}^{-1}
\end{array}\right]
$$




$$
\boldsymbol{M}_{\beta}=\boldsymbol{K}_{\beta}^{-1}-\boldsymbol{A}_{\beta} \quad \text { e } \quad \boldsymbol{M}_{\lambda}=\boldsymbol{K}_{\lambda}^{-1}-\boldsymbol{A}_{\lambda}
$$

Os coeficientes $A_{1}, A_{2}$ e $A_{3}$ são agora obtidos substituindo os cumulantes do Apêndice A nas expressões (3.4), (3.5) e (3.6). Para facilitar os cálculos, escrevemos $A_{1}=A_{11}+A_{12}+$ $A_{13}+A_{14}$ e $A_{2}=A_{21}+A_{22}+A_{23}$. Temos então

$$
\begin{gathered}
A_{11}=3 \sum_{\beta, \lambda} \kappa_{r s t} \kappa_{u v w}\left[m^{r s} a^{v w}\left(m^{t u}+2 a^{t u}\right)+a^{r s} m^{t u} a^{v w}+2 m^{r u} a^{s v} a^{t w}\right], \\
A_{12}=-12 \sum_{\beta, \lambda} \kappa_{r s}^{(t)} \kappa_{u v}^{(w)}\left(\kappa^{r t} \kappa^{s u} \kappa^{v w}+a^{r t} a^{s u} a^{v w}+\kappa^{t u} \kappa^{r v} \kappa^{s w}+a^{t u} a^{r v} a^{s w}\right), \\
A_{13}=-6 \sum_{\beta, \lambda} \kappa_{r s t} \kappa_{u v}^{(w)}\left[\left(a^{t w}-\kappa^{t w}\right)\left(\kappa^{r u} \kappa^{s v}-a^{r u} a^{s v}\right)+m^{r s}\left(a^{t u} a^{v w}+\kappa^{t u} \kappa^{v w}\right)\right. \\
\left.\quad+2 a^{s t}\left(\kappa^{r u} \kappa^{v w}-a^{r u} a^{v w}\right)+2 a^{s u} a^{t v} m^{r w}\right], \\
A_{14}=6 \sum_{\beta, \lambda} \kappa_{r s t u} m^{r s} a^{t u}-6 \sum_{\beta, \lambda} \kappa_{r s t}^{(u)}\left[m^{r s}\left(a^{t u}-\kappa^{t u}\right)+2 m^{r u} a^{s t}\right] \\
+12 \sum_{\beta, \lambda} \kappa_{s t}^{(r u)}\left(\kappa^{r s} \kappa^{t u}-a^{r s} a^{t u}\right),
\end{gathered}
$$

$$
\begin{gathered}
A_{21}=-3 \sum_{\beta, \lambda} \kappa_{r s t} \kappa_{u v w}\left[\frac{1}{4} m^{r s} m^{t u}\left(4 a^{v w}+3 m^{w v}\right)+m^{r s} a^{t u} m^{v w}+\frac{1}{2} m^{r u} m^{s v}\left(4 a^{t w}+m^{t w}\right)\right], \\
A_{22}=6 \sum_{\beta, \lambda} \kappa_{r s t} \kappa_{u v}^{(w)}\left[m^{t w}\left(\kappa^{r u} \kappa^{s v}-a^{r u} a^{s v}\right)+m^{r s}\left(\kappa^{t u} \kappa^{v w}-a^{t u} a^{v w}\right)\right], \\
A_{23}=6 \sum_{\beta, \lambda} \kappa_{r s t}^{(u)} m^{r s} m^{t u}-3 \sum_{\beta, \lambda} \kappa_{r s t u} m^{r s} m^{t u}
\end{gathered}
$$

e

$$
A_{3}=\frac{3}{4} \sum_{\beta, \lambda} \kappa_{r s t} \kappa_{u v w} m^{r s} m^{t u} m^{v w}+\frac{1}{2} \sum_{\beta, \lambda} \kappa_{r s t} \kappa_{u v w} m^{r u} m^{s v} m^{t w}
$$


Apresentaremos com detalhes a obtenção da quantidade $A_{3}$, as demais quantidades são obtidas de maneira análoga e serão omitidas aqui. Temos então,

$$
\begin{aligned}
A_{3}= & \frac{3}{4} \sum_{\beta} \kappa_{r s t} \kappa_{u v w} m^{r s} m^{t u} m^{v w}+\frac{1}{2} \sum_{\beta} \kappa_{r s t} \kappa_{u v w} m^{r u} m^{s v} m^{t w} \\
& \frac{3}{4} \sum_{\beta, \lambda} \kappa_{r s t} \kappa_{u V W} m^{r s} m^{t u} m^{V W}+\frac{1}{2} \sum_{\beta, \lambda} \kappa_{r s T} \kappa_{u v W} m^{r u} m^{s v} m^{T W} \\
& \frac{3}{4} \sum_{\beta, \lambda} \kappa_{r s T} \kappa_{U v w} m^{r s} m^{T U} m^{v w}+\frac{1}{2} \sum_{\beta, \lambda} \kappa_{r S t} \kappa_{u V w} m^{r u} m^{S V} m^{t w} \\
& \frac{3}{4} \sum_{\beta, \lambda} \kappa_{R S t} \kappa_{u v w} m^{R S} m^{t u} m^{v w}+\frac{1}{2} \sum_{\beta, \lambda} \kappa_{R s t} \kappa_{U v w} m^{R U} m^{s v} m^{t w} \\
& \frac{3}{4} \sum_{\beta, \lambda} \kappa_{r s T} \kappa_{U V W} m^{r s} m^{T U} m^{V W}+\frac{1}{2} \sum_{\beta, \lambda} \kappa_{r S T} \kappa_{u V W} m^{r u} m^{S V} m^{T W} \\
& \frac{3}{4} \sum_{\beta, \lambda} \kappa_{R S t} \kappa_{u V W} m^{R S} m^{t u} m^{V W}+\frac{1}{2} \sum_{\beta, \lambda} \kappa_{R s T} \kappa_{U v W} m^{R U} m^{s v} m^{T W} \\
& \frac{3}{4} \sum_{\beta, \lambda} \kappa_{R S T} \kappa_{U v w} m^{R S} m^{T U} m^{v w}+\frac{1}{2} \sum_{\beta, \lambda} \kappa_{R S t} \kappa_{U V w} m^{R U} m^{S V} m^{t w} \\
& \frac{3}{4} \sum_{\lambda} \kappa_{R S T} \kappa_{U V W} m^{R S} m^{T U} m^{V W}+\frac{1}{2} \sum_{\lambda} \kappa_{R S T} \kappa_{U V W} m^{R U} m^{S V} m^{T W} .
\end{aligned}
$$

Substituindo os cumulantes do Apêndice A na expressão anterior, obtemos 


$$
\begin{aligned}
A_{3}= & \sum_{\beta}\left\{-\sum_{\ell=1}^{n} \phi_{\ell}\left(f_{\ell}+2 g_{\ell}\right) x_{\ell r} x_{\ell s} x_{\ell t}\right\}\left\{-\sum_{m=1}^{n} \phi_{m}\left(f_{m}+2 g_{m}\right) x_{m u} x_{m v} x_{m w}\right\} \\
& \times\left(\frac{3}{4} m^{r s} m^{t u} m^{v w}+\frac{1}{2} m^{r u} m^{s v} m^{t w}\right) \\
& +\frac{1}{2} \sum_{\beta, \lambda}\left\{-\sum_{\ell=1}^{n} \phi_{1 \ell} w_{\ell} x_{\ell r} x_{\ell s} s_{\ell T}\right\}\left\{-\sum_{m=1}^{n} \phi_{1 m} w_{m} x_{m u} x_{m v} s_{m W}\right\} m^{r u} m^{s v} m^{T W} \\
& +\frac{3}{4} \sum_{\beta, \lambda}\left\{-\sum_{\ell=1}^{n} \phi_{1 \ell} w_{\ell} x_{\ell r} x_{\ell s} s_{\ell T}\right\}\left\{-\sum_{m=1}^{n} \phi_{1 m} w_{m} s_{m U} x_{m v} x_{m w}\right\} m^{r s} m^{T U} m^{v w} \\
& +\frac{1}{2} \sum_{\beta, \lambda}\left\{-\sum_{\ell=1}^{n} \phi_{1 \ell} w_{\ell} x_{\ell r} s_{\ell S} x_{\ell t}\right\}\left\{-\sum_{m=1}^{n} \phi_{1 m} w_{m} x_{m u} s_{m V} x_{m w}\right\} m^{r u} m^{S V} m^{t w} \\
+ & \frac{1}{2} \sum_{\beta, \lambda}\left\{-\sum_{\ell=1}^{n} \phi_{1 \ell} w_{\ell} s_{\ell R} x_{\ell s} x_{\ell t}\right\}\left\{-\sum_{m=1}^{n} \phi_{1 m} w_{m} s_{m U} x_{m v} x_{m w}\right\} m^{R U} m^{s v} m^{t w} \\
+ & \frac{3}{4} \sum_{\beta, \lambda}\left\{-\sum_{\ell=1}^{n} \phi_{1 \ell} w_{\ell} x_{\ell r} x_{\ell s} s_{\ell T}\right\}\left\{\sum_{m=1}^{n}\left(d_{3 m} \phi_{1 m}^{3}+3 d_{2 m} \phi_{1 m} \phi_{2 m}\right) s_{\ell U} s_{\ell V} s_{\ell W}\right\} m^{r s} m^{T U} m^{V W} \\
+ & \frac{3}{4} \sum_{\beta, \lambda}\left\{\sum_{\ell=1}^{n}\left(d_{3 \ell} \phi_{1 \ell}^{3}+3 d_{2 \ell} \phi_{1 \ell} \phi_{2 \ell}\right) s_{\ell R} s_{\ell S} s_{\ell T}\right\}\left\{-\sum_{m=1}^{n} \phi_{1 m} w_{m} s_{m U} x_{m v} s_{m w}\right\} m^{R S} m^{T U} m^{v w} \\
+ & \sum_{\lambda}\left\{\sum_{\ell=1}^{n}\left(d_{3 \ell} \phi_{1 \ell}^{3}+3 d_{2 \ell} \phi_{1 \ell} \phi_{2 \ell}\right) s_{\ell R} s_{\ell S} s_{\ell T}\right\}\left\{\sum_{\ell=1}^{n}\left(d_{3 m} \phi_{1 m}^{3}+3 d_{2 m} \phi_{1 m} \phi_{2 m}\right) s_{m U} s_{m V} s_{m W}\right\} \\
& \times\left(\frac{3}{4} m^{R S} m^{T U} m^{V W}+\frac{1}{2} m^{R U} m^{S V} m^{T W}\right) \cdot
\end{aligned}
$$

Invertendo a ordem dos somatórios e rearranjando os termos, obtemos 


$$
\begin{aligned}
& A_{3}=\sum_{\ell, m=1}^{n}\left\{[ \phi _ { \ell } ( f _ { \ell } + 2 g _ { \ell } ) ] \left[\frac{3}{4}\left(\sum_{r, s=1}^{p} x_{\ell r} m^{r s} x_{s \ell}\right)\left(\sum_{t, u=1}^{p} x_{\ell t} m^{t u} x_{u m}\right)\left(\sum_{v, w=1}^{p} x_{m v} m^{v w} x_{w m}\right)\right.\right. \\
& \left.\left.+\frac{1}{2}\left(\sum_{r, u=1}^{p} x_{\ell r} m^{r u} x_{u m}\right)\left(\sum_{s, v=1}^{p} x_{\ell s} m^{s v} x_{v m}\right)\left(\sum_{t, w=1}^{p} x_{\ell t} m^{t w} x_{w m}\right)\right]\left[\phi_{m}\left(f_{m}+2 g_{m}\right)\right]\right\} \\
& +\sum_{\ell, m=1}^{n}\left\{( \phi _ { 1 \ell } w _ { \ell } ) \left[\frac{3}{4}\left(\sum_{r, s=1}^{p} x_{\ell r} m^{r s} x_{s \ell}\right)\left(\sum_{T, U=1}^{q} s_{\ell T} m^{T U} s_{U m}\right)\left(\sum_{v, w=1}^{p} x_{m v} m^{v w} x_{w m}\right)\right.\right. \\
& \left.\left.+\frac{3}{2}\left(\sum_{r, u=1}^{p} x_{\ell r} m^{r u} x_{u m}\right)\left(\sum_{s, v=1}^{p} x_{\ell s} m^{s v} x_{v m}\right)\left(\sum_{T, W=1}^{q} s_{\ell T} m^{T W} s_{W m}\right)\right]\left(\phi_{1 m} w_{m}\right)\right\} \\
& -\frac{3}{4} \sum_{\ell, m=1}^{n}\left\{\left(\phi_{1 \ell} w_{\ell}\right)\left(\sum_{r, s=1}^{p} x_{\ell r} m^{r s} x_{s \ell}\right)\left(\sum_{T, U=1}^{q} s_{\ell T} m^{T U} s_{U m}\right)\left(\sum_{V, W=1}^{q} s_{m V} m^{V W} s_{W m}\right)\right. \\
& \left.\times\left(d_{3 m} \phi_{1 m}^{3}+3 d_{2 m} \phi_{1 m} \phi_{2 m}\right)\right\} \\
& -\frac{3}{4} \sum_{\ell, m=1}^{n}\left\{\left(d_{3 m} \phi_{1 m}^{3}+3 d_{2 m} \phi_{1 m} \phi_{2 m}\right)\left(\sum_{R, S=1}^{q} s_{\ell R} m^{R S} s_{S \ell}\right)\left(\sum_{T, U=1}^{q} s_{\ell T} m^{T U} s_{U m}\right)\right. \\
& \left.\times\left(\sum_{v, w=1}^{p} x_{m v} m^{v w} x_{w m}\right)\left(\phi_{1 \ell} w_{\ell}\right)\right\} \\
& +\sum_{\ell, m=1}^{n}\left\{( d _ { 3 \ell } \phi _ { 1 \ell } ^ { 3 } + 3 d _ { 2 \ell } \phi _ { 1 \ell } \phi _ { 2 \ell } ) \left[\frac{3}{4}\left(\sum_{R, S=1}^{q} s_{\ell R} m^{R S} s_{S \ell}\right)\left(\sum_{T, U=1}^{q} s_{\ell T} m^{T U} s_{S m}\right)\right.\right. \\
& \times\left(\sum_{V, W=1}^{q} s_{m V} m^{V W} s_{W m}\right)+\frac{1}{2}\left(\sum_{R, S=1}^{q} s_{\ell R} m^{R S} s_{S \ell}\right)\left(\sum_{T, U=1}^{q} s_{\ell T} m^{T U} s_{S m}\right) \\
& \left.\left.\times\left(\sum_{V, W=1}^{q} s_{m V} m^{V W} s_{W m}\right)\right]\left(d_{3 m} \phi_{1 m}^{3}+3 d_{2 m} \phi_{1 m} \phi_{2 m}\right)\right\} .
\end{aligned}
$$

Em notação matricial temos,

$$
\begin{aligned}
A_{3}= & \mathbf{1}^{\top} \boldsymbol{\Phi}(\boldsymbol{F}+2 \boldsymbol{G})\left[\frac{3}{4}\left(\boldsymbol{Z}_{\beta d}-\boldsymbol{Z}_{\beta_{1} d}\right)\left(\boldsymbol{Z}_{\beta}-\boldsymbol{Z}_{\beta_{1}}\right)\left(\boldsymbol{Z}_{\beta d}-\boldsymbol{Z}_{\beta_{1} d}\right)+\frac{1}{2}\left(\boldsymbol{Z}_{\beta}-\boldsymbol{Z}_{\beta_{1}}\right)^{(3)}\right] \boldsymbol{\Phi}(\boldsymbol{F}+2 \boldsymbol{G}) \mathbf{1} \\
+ & \mathbf{1}^{\top} \boldsymbol{\Phi}_{1} \boldsymbol{W}\left[\frac{3}{4}\left(\boldsymbol{Z}_{\beta d}-\boldsymbol{Z}_{\beta_{1} d}\right)\left(\boldsymbol{Z}_{\lambda}-\boldsymbol{Z}_{\lambda_{1}}\right)\left(\boldsymbol{Z}_{\beta d}-\boldsymbol{Z}_{\beta_{1} d}\right)+\frac{3}{2}\left(\boldsymbol{Z}_{\beta}-\boldsymbol{Z}_{\beta_{1}}\right)^{(2)} \odot\left(\boldsymbol{Z}_{\lambda}-\boldsymbol{Z}_{\lambda_{1}}\right)\right] \boldsymbol{\Phi}_{1} \boldsymbol{W} \mathbf{1} \\
& -\frac{3}{2} \mathbf{1}^{\top} \boldsymbol{\Phi}_{1} \boldsymbol{W}\left(\boldsymbol{Z}_{\beta d}-\boldsymbol{Z}_{\beta_{1} d}\right)\left(\boldsymbol{Z}_{\lambda}-\boldsymbol{Z}_{\lambda_{1}}\right)\left(\boldsymbol{Z}_{\lambda d}-\boldsymbol{Z}_{\lambda_{1} d}\right)\left(\boldsymbol{D}_{3} \boldsymbol{\Phi}_{1}^{3}+3 \boldsymbol{D}_{2} \boldsymbol{\Phi}_{1} \boldsymbol{\Phi}_{2}\right) \mathbf{1} \\
+ & \mathbf{1}^{\top}\left(\boldsymbol{D}_{3} \boldsymbol{\Phi}_{1}^{3}+3 \boldsymbol{D}_{2} \boldsymbol{\Phi}_{1} \boldsymbol{\Phi}_{2}\right)\left[\frac{3}{4}\left(\boldsymbol{Z}_{\lambda d}-\boldsymbol{Z}_{\lambda_{1} d}\right)\left(\boldsymbol{Z}_{\lambda}-\boldsymbol{Z}_{\lambda_{1}}\right)\left(\boldsymbol{Z}_{\lambda d}-\boldsymbol{Z}_{\lambda_{1} d}\right)+\frac{1}{2}\left(\boldsymbol{Z}_{\lambda}-\boldsymbol{Z}_{\lambda_{1}}\right)^{(3)}\right] \\
& \quad \times\left(\boldsymbol{D}_{3} \boldsymbol{\Phi}_{1}^{3}+3 \boldsymbol{D}_{2} \boldsymbol{\Phi}_{1} \boldsymbol{\Phi}_{2}\right) \mathbf{1} .
\end{aligned}
$$




\section{Apêndice C}

\section{Obtenção das quantidades $A_{40}, A_{32}, A_{33}$ \\ e $A_{22}$ do coeficiente de curtose para a distribuição do $\operatorname{EMV} \hat{\boldsymbol{\theta}}=\left(\hat{\boldsymbol{\beta}}^{\top}, \hat{\boldsymbol{\lambda}}^{\top}\right)^{\top}$}

Neste apêndice apresentamos a obtenção das quantidades $A_{40}, A_{32}$ e $A_{33}$ e $A_{22}$ referente ao coeficiente de curtose da distribuição do $\operatorname{EMV} \hat{\boldsymbol{\theta}}=\left(\hat{\boldsymbol{\beta}}^{\top}, \hat{\boldsymbol{\lambda}}^{\top}\right)^{\top}$.

Como a obtenção dessas quantidades requer uma álgebra longa e tediosa, apresentaremos apenas os detalhes para a obtenção de $A_{32}$, as demais quantidades são obtidas de maneira análoga e serão omitidas aqui.

C.1 Obtenção de $A_{32}$ para o coeficiente de curtose da distribuição de $\hat{\boldsymbol{\beta}}$

Para facilitar os cálculos, escrevemos $A_{32 \beta}=A_{32 \beta}^{1}+A_{32 \beta}^{2}+A_{32 \beta}^{3}+A_{32 \beta}^{4}$. Temos então da expressão (5.3) que

$$
\begin{gathered}
A_{32 \beta}^{1}=2 \sum_{\beta, \lambda} \kappa^{a, r} \kappa^{s, t} \kappa^{a, u} \kappa^{a, v} \kappa^{a, w}\left(\kappa_{r s, t} \kappa_{u, v, w}+\kappa_{r s, u} \kappa_{t, v, w}+\kappa_{r s, v} \kappa_{t, u, w}+\kappa_{r s, w} \kappa_{t, u, v}\right) \\
A_{32 \beta}^{2}=6 \sum_{\beta, \lambda} \kappa^{a, r} \kappa^{a, u}\left(\kappa^{a, s} \kappa^{a, t}+\kappa^{a, a} \kappa^{s, t}\right) \kappa_{r s, t, u} \\
A_{32 \beta}^{3}=\sum_{\beta, \lambda} \kappa^{a, r} \kappa^{a, w}\left(\kappa^{s, t} \kappa^{a, u} \kappa^{a, v}+6 \kappa^{a, s} \kappa^{t, u} \kappa^{a, v}+3 \kappa^{a, a} \kappa^{s, u} \kappa^{t, v}\right) \kappa_{r s t} \kappa_{u, v, w}
\end{gathered}
$$


e

$$
A_{32 \beta}^{4}=-\left[\sum_{\beta, \lambda} \kappa^{a, r} \kappa^{s, t}\left(2 \kappa_{r s, t}+\kappa_{r s t}\right)\right]\left[\sum_{\beta, \lambda} \kappa^{a, u} \kappa^{a, v} \kappa^{a, w} \kappa_{u, v, w}\right] .
$$

Desenvolvendo as expressões anteriores, obtemos

$$
\begin{aligned}
A_{32 \beta}^{1}= & 2 \sum_{\beta} \kappa^{a, r} \kappa^{s, t} \kappa^{a, u} \kappa^{a, v} \kappa^{a, w}\left(\kappa_{r s, t} \kappa_{u, v, w}+\kappa_{r s, u} \kappa_{t, v, w}+\kappa_{r s, v} \kappa_{t, u, w}+\kappa_{r s, w} \kappa_{t, u, v}\right) \\
& +2 \sum_{\beta, \lambda} \kappa^{a, r} \kappa^{S, T} \kappa^{a, u} \kappa^{a, v} \kappa^{a, w}\left(\kappa_{r S, T} \kappa_{u, v, w}+\kappa_{r S, u} \kappa_{T, v, w}+\kappa_{r S, v} \kappa_{T, u, w}+\kappa_{r S, w} \kappa_{T, u, v}\right) \\
& A_{32 \beta}^{2}=6 \sum_{\beta} \kappa^{a, r} \kappa^{a, u} \kappa^{a, s} \kappa^{a, t} \kappa_{r s, t, u}+6 \sum_{\beta} \kappa^{a, r} \kappa^{a, u} \kappa^{a, a} \kappa^{s, t} \kappa_{r s, t, u} \\
& +6 \sum_{\beta, \lambda} \kappa^{a, r} \kappa^{a, u} \kappa^{a, a} \kappa^{S, T} \kappa_{r S, T, u}, \\
A_{32 \beta}^{3}= & \sum_{\beta} \kappa^{a, r} \kappa^{a, w} \kappa^{s, t} \kappa^{a, u} \kappa^{a, v} \kappa_{r s t} \kappa_{u, v, w}+6 \sum_{\beta} \kappa^{a, r} \kappa^{a, w} \kappa^{a, s} \kappa^{t, u} \kappa^{a, v} \kappa_{r s t} \kappa_{u, v, w} \\
& +3 \sum_{\beta} \kappa^{a, r} \kappa^{a, w} \kappa^{a, a} \kappa^{s, u} \kappa^{t, v} \kappa_{r s t} \kappa_{u, v, w}+\sum_{\beta, \lambda} \kappa^{a, r} \kappa^{a, w} \kappa^{S, T} \kappa^{a, u} \kappa^{a, v} \kappa_{r S T} \kappa_{u, v, w} \\
& +6 \sum_{\beta, \lambda} \kappa^{a, r} \kappa^{a, w} \kappa^{a, s} \kappa^{T, U} \kappa^{a, v} \kappa_{r s T} \kappa_{U, v, w}+3 \sum_{\beta, \lambda} \kappa^{a, r} \kappa^{a, w} \kappa^{a, a} \kappa^{S, U} \kappa^{t, v} \kappa_{r S t} \kappa_{U, v, w} \\
& +3 \sum_{\beta, \lambda} \kappa^{a, r} \kappa^{a, w} \kappa^{a, a} \kappa^{s, u} \kappa^{T, V} \kappa_{r s T} \kappa_{u, V, w}+3 \sum_{\beta, \lambda} \kappa^{a, r} \kappa^{a, w} \kappa^{a, a} \kappa^{S, U} \kappa^{T, V} \kappa_{r S T} \kappa_{U, V, w}
\end{aligned}
$$

e

$$
\begin{aligned}
A_{32 \beta}^{4}= & -\left[\sum_{\beta} \kappa^{a, r} \kappa^{s, t}\left(2 \kappa_{r s, t}+\kappa_{r s t}\right)\right]\left[\sum_{\beta} \kappa^{a, u} \kappa^{a, v} \kappa^{a, w} \kappa_{u, v, w}\right] \\
& -\left[\sum_{\beta, \lambda} \kappa^{a, r} \kappa^{S, T}\left(2 \kappa_{r S, T}+\kappa_{r S T}\right)\right]\left[\sum_{\beta} \kappa^{a, u} \kappa^{a, v} \kappa^{a, w} \kappa_{u, v, w}\right] .
\end{aligned}
$$


Substituindo os cumulantes do Apêndice A, invertendo a ordem dos somatórios e rearranjando os termos, obtemos

$$
\begin{aligned}
A_{32 \beta}^{1}= & 2 \sum_{\ell=1}^{n}\left\{\left(\sum_{r=1}^{p} \kappa^{a, r} x_{r \ell}\right)\left(\sum_{s, t=1}^{p} x_{\ell s} \kappa^{s, t} x_{t \ell}\right)\left(\phi_{\ell} g_{\ell}\right)\right\} \\
& \times \sum_{m=1}^{n}\left\{\left(\sum_{u=1}^{p} \kappa^{a, u} x_{u m}\right)\left(\sum_{v=1}^{p} \kappa^{a, v} x_{v m}\right)\left(\sum_{w=1}^{p} \kappa^{a, w} x_{w m}\right)\left[\phi_{m}\left(f_{m}-g_{m}\right)\right]\right\} \\
& +6 \sum_{\ell, m=1}^{n}\left\{\left(\sum_{r=1}^{p} \kappa^{a, r} x_{r \ell}\right)\left(\sum_{u=1}^{p} \kappa^{a, u} x_{u \ell}\right)\left(\phi_{\ell} g_{\ell}\right)\left(\sum_{s, t=1}^{p} x_{\ell s} \kappa^{s, t} x_{t m}\right)\right. \\
& \left.\times\left[\phi_{m}\left(f_{m}-g_{m}\right)\right]\left(\sum_{v=1}^{p} x_{m v} \kappa^{v, a}\right)\left(\sum_{w=1}^{p} x_{m w} \kappa^{w, a}\right)\right\} \\
& +6 \sum_{\ell, m=1}^{n}\left\{\left(\sum_{r=1}^{p} \kappa^{a, r} x_{r \ell}\right)\left(\sum_{u=1}^{p} \kappa^{a, u} x_{u \ell}\right)\left(\phi_{1 \ell} w_{\ell}\right)\left(\sum_{S, T=1}^{q} s_{\ell S} \kappa^{S, T} s_{T m}\right)\right. \\
& \left.\times\left(-\phi_{1 m} w_{m}\right)\left(\sum_{v=1}^{p} x_{m v} \kappa^{v, a}\right)\left(\sum_{w=1}^{p} x_{m w} \kappa^{w, a}\right)\right\}, \\
A_{32 \beta}^{2}=6 & \sum_{\ell=1}^{n}\left\{\left(\sum_{r=1}^{p} \kappa^{a, r} x_{r \ell}\right)\left(\sum_{s=1}^{p} \kappa^{a, s} x_{s \ell}\right)\left(\phi_{\ell} m_{\ell}\right)\left(\sum_{t=1}^{p} x_{\ell t} \kappa^{t, a}\right)\left(\sum_{u=1}^{p} x_{\ell u} \kappa^{u, a}\right)\right\} \\
+ & 6 \kappa^{a, a} \sum_{\ell=1}^{n}\left\{\left(\sum_{r=1}^{p} \kappa^{a, r} x_{r \ell}\right)\left(\phi_{\ell} m_{\ell}\right)\left(\sum_{s, t=1}^{p} x_{\ell s} \kappa^{s, t} x_{t \ell}\right)\left(\sum_{u=1}^{n} x_{\ell u} \kappa^{u, a}\right)\right\} \\
& \left\{\left(\sum_{r=1}^{p} \kappa^{a, r} x_{r \ell}\right)\left(-\phi_{1 \ell}^{2} \phi_{\ell}^{-1} w_{\ell}\right)\left(\sum_{S, T=1}^{q} x_{\ell S} \kappa^{S, T} s_{T \ell}\right)\left(\sum_{u=1}^{p} x_{\ell u} \kappa^{u, a}\right)\right\}
\end{aligned}
$$




$$
\begin{aligned}
& A_{32 \beta}^{3}=\sum_{\ell=1}^{n}\left\{\left(\sum_{r=1}^{p} \kappa^{a, r} x_{r \ell}\right)\left(\sum_{s, t=1}^{p} x_{\ell s} \kappa^{s, t} x_{t \ell}\right)\left[-\phi_{\ell}\left(f_{\ell}+2 g_{\ell}\right)\right]\right\} \\
& \times \sum_{m=1}^{n}\left\{\left(\sum_{u=1}^{p} x_{m u} \kappa^{u, a}\right)\left(\sum_{v=1}^{p} x_{m v} \kappa^{v, a}\right)\left(\sum_{w=1}^{p} x_{m w} \kappa^{w, a}\right)\left[\phi_{m}\left(f_{m}-g_{m}\right)\right]\right\} \\
& +6 \sum_{\ell, m=1}^{n}\left\{\left(\sum_{r=1}^{p} \kappa^{a, r} x_{r \ell}\right)\left(\sum_{s=1}^{p} \kappa^{a, s} x_{s \ell}\right)\left[-\phi_{\ell}\left(f_{\ell}+2 g_{\ell}\right)\right]\left(\sum_{t, u=1}^{p} x_{\ell t} \kappa^{t, u} x_{u m}\right)\right. \\
& \left.\times\left[\phi_{m}\left(f_{m}-g_{m}\right)\right]\left(\sum_{v=1}^{p} x_{m v} \kappa^{v, a}\right)\left(\sum_{w=1}^{p} x_{m w} \kappa^{w, a}\right)\right\} \\
& +3 \kappa^{a, a} \sum_{\ell=1}^{n}\left\{\left(\sum_{r=1}^{p} \kappa^{a, r} x_{r \ell}\right)\left[-\phi_{\ell}\left(f_{\ell}+2 g_{\ell}\right)\right]\left(\sum_{s, u=1}^{p} x_{\ell s} \kappa^{s, u} x_{u m}\right)\left(\sum_{t, v=1}^{p} x_{\ell t} \kappa^{t, v} x_{v m}\right)\right. \\
& \left.\times\left[\phi_{m}\left(f_{m}-g_{m}\right)\right]\left(\sum_{w=1}^{p} x_{m w} \kappa^{w, a}\right)\right\} \\
& +6 \sum_{\ell, m=1}^{n}\left\{\left(\sum_{r=1}^{p} \kappa^{a, r} x_{r \ell}\right)\left(\sum_{s=1}^{p} \kappa^{a, s} x_{s \ell}\right)\left(-\phi_{1 \ell} w_{\ell}\right)\left(\sum_{T, U=1}^{q} s_{\ell T} \kappa^{T, U} s_{U m}\right)\right. \\
& \left.\times\left(-\phi_{1 \ell} w_{\ell}\right)\left(\sum_{v=1}^{p} x_{m v} \kappa^{v, a}\right)\left(\sum_{w=1}^{p} x_{m w} \kappa^{w, a}\right)\right\} \\
& +3 \kappa^{a, a} \sum_{\ell=1}^{n}\left\{\left(\sum_{r=1}^{p} \kappa^{a, r} x_{r \ell}\right)\left(-\phi_{1 \ell} w_{\ell}\right)\left(\sum_{S, U=1}^{q} s_{\ell S} \kappa^{S, U} s_{U m}\right)\left(\sum_{t, v=1}^{p} x_{\ell t} \kappa^{t, v} x_{v m}\right)\right. \\
& \left.\times\left(-\phi_{1 \ell} w_{\ell}\right)\left(\sum_{w=1}^{p} x_{m w} \kappa^{w, a}\right)\right\} \\
& +3 \kappa^{a, a} \sum_{\ell=1}^{n}\left\{\left(\sum_{r=1}^{p} \kappa^{a, r} x_{r \ell}\right)\left(-\phi_{1 \ell} w_{\ell}\right)\left(\sum_{s, u=1}^{p} x_{\ell s} \kappa^{s, u} x_{u m}\right)\left(\sum_{T, V=1}^{q} s_{\ell T} \kappa^{T, V} s_{V m}\right)\right. \\
& \left.\times\left(-\phi_{1 \ell} w_{\ell}\right)\left(\sum_{w=1}^{p} x_{m w} \kappa^{w, a}\right)\right\},
\end{aligned}
$$

$\mathrm{e}$

$$
\begin{aligned}
A_{32 \beta}^{4}= & -\sum_{\ell=1}^{n}\left\{\left(\sum_{r=1}^{p} \kappa^{a, r} x_{r \ell}\right)\left(\sum_{s, t=1}^{p} x_{\ell s} \kappa^{s, t} x_{t \ell}\right)\left(-\phi_{\ell} f_{\ell}\right)\right\} \\
& \times \sum_{m=1}^{n}\left\{\left(\sum_{u=1}^{p} \kappa^{a, u} x_{u m}\right)\left(\sum_{v=1}^{p} \kappa^{a, v} x_{v m}\right)\left(\sum_{w=1}^{p} \kappa^{a, w} x_{w m}\right)\left[\phi_{m}\left(f_{m}-g_{m}\right)\right]\right\} .
\end{aligned}
$$


Escrevendo as expressões em notação matricial, obtemos

$$
\begin{aligned}
& \mathbf{A}_{\mathbf{3 2} \beta}^{\mathbf{1}}=2\left[\boldsymbol{P}_{\beta} \boldsymbol{Z}_{\beta d} \boldsymbol{\Phi} \boldsymbol{G} \mathbf{1}\right]\left[\left(\boldsymbol{P}_{\beta} \odot \boldsymbol{P}_{\beta} \odot \boldsymbol{P}_{\beta}\right) \boldsymbol{\Phi}(\boldsymbol{F}-\boldsymbol{G}) \mathbf{1}\right]^{\top} \\
& +6\left[\left(\boldsymbol{P}_{\beta} \odot \boldsymbol{P}_{\beta}\right) \boldsymbol{\Phi} \boldsymbol{G} \boldsymbol{Z}_{\beta} \boldsymbol{\Phi}(\boldsymbol{F}-\boldsymbol{G})\left(\boldsymbol{P}_{\beta} \odot \boldsymbol{P}_{\beta}\right)^{\top}\right]_{d} \\
& -6\left[\left(\boldsymbol{P}_{\beta} \odot \boldsymbol{P}_{\beta}\right) \boldsymbol{\Phi}_{1} \boldsymbol{W} \boldsymbol{Z}_{\lambda} \boldsymbol{\Phi}_{1} \boldsymbol{W}\left(\boldsymbol{P}_{\beta} \odot \boldsymbol{P}_{\beta}\right)^{\top}\right]_{d}, \\
& \mathbf{A}_{\mathbf{3 2} \beta}^{\mathbf{2}}=6\left[\left(\boldsymbol{P}_{\beta} \odot \boldsymbol{P}_{\beta}\right) \boldsymbol{\Phi} \boldsymbol{M}\left(\boldsymbol{P}_{\beta} \odot \boldsymbol{P}_{\beta}\right)^{\top}\right]_{d} \\
& +6\left[\boldsymbol{K}_{\beta}^{-1}\right]_{d}\left[\boldsymbol{P}_{\beta} \boldsymbol{\Phi} \boldsymbol{M} \boldsymbol{Z}_{\beta d} \boldsymbol{P}_{\beta}^{\top}\right]_{d} \\
& -6\left[\boldsymbol{K}_{\beta}^{-1}\right]_{d}\left[\boldsymbol{P}_{\beta} \boldsymbol{\Phi}_{1}^{2} \boldsymbol{\Phi}^{-1} \boldsymbol{W} \boldsymbol{Z}_{\lambda d} \boldsymbol{P}_{\beta}^{\top}\right]_{d}, \\
& \mathbf{A}_{\mathbf{3 2} \beta}^{\mathbf{3}}=-\left[\boldsymbol{P}_{\beta} \boldsymbol{Z}_{\beta d} \boldsymbol{\Phi}(\boldsymbol{F}+2 \boldsymbol{G}) \mathbf{1}\right]\left[\left(\boldsymbol{P}_{\beta} \odot \boldsymbol{P}_{\beta} \odot \boldsymbol{P}_{\beta}\right) \boldsymbol{\Phi}(\boldsymbol{F}-\boldsymbol{G}) \mathbf{1}\right]^{\top} \\
& -6\left[\left(\boldsymbol{P}_{\beta} \odot \boldsymbol{P}_{\beta}\right) \boldsymbol{\Phi}(\boldsymbol{F}+2 \boldsymbol{G}) \boldsymbol{Z}_{\beta} \boldsymbol{\Phi}(\boldsymbol{F}-\boldsymbol{G})\left(\boldsymbol{P}_{\beta} \odot \boldsymbol{P}_{\beta}\right)^{\top}\right]_{d} \\
& -3\left[\boldsymbol{K}_{\beta}^{-1}\right]_{d}\left[\boldsymbol{P}_{\beta} \boldsymbol{\Phi}(\boldsymbol{F}+2 \boldsymbol{G})\left(\boldsymbol{Z}_{\beta} \odot \boldsymbol{Z}_{\beta}\right) \boldsymbol{\Phi}(\boldsymbol{F}-\boldsymbol{G}) \boldsymbol{P}_{\beta}^{\top}\right]_{d} \\
& +6\left[\left(\boldsymbol{P}_{\beta} \odot \boldsymbol{P}_{\beta}\right) \boldsymbol{\Phi}_{1} \boldsymbol{W} \boldsymbol{Z}_{\lambda} \boldsymbol{\Phi}_{1} \boldsymbol{W}\left(\boldsymbol{P}_{\beta} \odot \boldsymbol{P}_{\beta}\right)^{\top}\right]_{d} \\
& +6\left[\boldsymbol{K}_{\beta}^{-1}\right]_{d}\left[\boldsymbol{P}_{\beta} \boldsymbol{\Phi}_{1} \boldsymbol{W}\left(\boldsymbol{Z}_{\beta} \odot \boldsymbol{Z}_{\lambda}\right) \boldsymbol{\Phi}_{1} \boldsymbol{W} \boldsymbol{P}_{\beta}^{\top}\right]_{d}
\end{aligned}
$$

$$
\mathbf{A}_{\mathbf{3 2} \beta}^{\mathbf{4}}=\left[\boldsymbol{P}_{\beta} \boldsymbol{Z}_{\beta d} \boldsymbol{\Phi} \boldsymbol{F} \mathbf{1}\right]\left[\left(\boldsymbol{P}_{\beta} \odot \boldsymbol{P}_{\beta} \odot \boldsymbol{P}_{\beta}\right) \boldsymbol{\Phi}(\boldsymbol{F}-\boldsymbol{G}) \mathbf{1}\right]^{\top}
$$

C.2 Obtenção de $A_{32}$ para o coeficiente de curtose da distribuição de $\hat{\lambda}$

Para facilitar os cálculos, escrevemos $A_{32 \lambda}=A_{32 \lambda}^{1}+A_{32 \lambda}^{2}+A_{32 \lambda}^{3}+A_{32 \lambda}^{4}$. Temos então da expressão (5.3) que

$$
\begin{gathered}
A_{32 \lambda}^{1}=2 \sum_{\beta, \lambda} \kappa^{A, R} \kappa^{S, T} \kappa^{A, U} \kappa^{A, V} \kappa^{A, W}\left(\kappa_{R S, T} \kappa_{U, V, W}+\kappa_{R S, U} \kappa_{T, V, W}\right. \\
\left.+\kappa_{R S, V} \kappa_{T, U, W}+\kappa_{R S, W} \kappa_{T, U, V}\right) \\
A_{32 \lambda}^{2}=6 \sum_{\beta, \lambda} \kappa^{A, R} \kappa^{A, U}\left(\kappa^{A, S} \kappa^{A, T}+\kappa^{A, A} \kappa^{S, T}\right) \kappa_{R S, T, U} \\
A_{32 \lambda}^{3}=\sum_{\beta, \lambda} \kappa^{A, R} \kappa^{A, W}\left(\kappa^{S, T} \kappa^{A, U} \kappa^{A, V}+6 \kappa^{A, S} \kappa^{T, U} \kappa^{A, V}+3 \kappa^{A, A} \kappa^{S, U} \kappa^{T, V}\right) \kappa_{R S T} \kappa_{U, V, W}
\end{gathered}
$$


e

$$
A_{32 \lambda}^{4}=-\left[\sum_{\beta, \lambda} \kappa^{A, R} \kappa^{S, T}\left(2 \kappa_{R S, T}+\kappa_{R S T}\right)\right]\left[\sum_{\beta, \lambda} \kappa^{A, U} \kappa^{A, V} \kappa^{A, W} \kappa_{U, V, W}\right]
$$

Desenvolvendo as expressões anteriores, obtemos

$$
\begin{aligned}
A_{32 \lambda}^{1}= & 2 \sum_{\lambda} \kappa^{A, R} \kappa^{S, T} \kappa^{A, U} \kappa^{A, V} \kappa^{A, W}\left(\kappa_{R S, T} \kappa_{U, V, W}+\kappa_{R S, U} \kappa_{T, V, W}+\kappa_{R S, V} \kappa_{T, U, W}+\kappa_{R S, W} \kappa_{T, U, V}\right) \\
& +2 \sum_{\beta, \lambda} \kappa^{A, R} \kappa^{s, t} \kappa^{A, U} \kappa^{A, V} \kappa^{A, W}\left(\kappa_{R s, t} \kappa_{U, V, W}+\kappa_{R s, U} \kappa_{t, V, W}+\kappa_{R s, V} \kappa_{t, U, W}+\kappa_{R s, W} \kappa_{t, U, V}\right), \\
& A_{32 \lambda}^{2}=6 \sum_{\lambda} \kappa^{A, R} \kappa^{A, U} \kappa^{A, S} \kappa^{A, T} \kappa_{R S, T, U}+6 \sum_{\lambda} \kappa^{A, R} \kappa^{A, U} \kappa^{A, A} \kappa^{S, T} \kappa_{R S, T, U} \\
& +6 \sum_{\beta, \lambda} \kappa^{A, R} \kappa^{A, U} \kappa^{A, A} \kappa^{s, t} \kappa_{R s, t, U}, \\
A_{32 \lambda}^{3}= & \sum_{\lambda} \kappa^{A, R} \kappa^{A, W} \kappa^{S, T} \kappa^{A, U} \kappa^{A, V} \kappa_{R S T} \kappa_{U, V, W}+6 \sum_{\lambda} \kappa^{A, R} \kappa^{A, W} \kappa^{A, S} \kappa^{T, U} \kappa^{A, V} \kappa_{R S T} \kappa_{U, V, W} \\
& +3 \sum_{\lambda} \kappa^{A, R} \kappa^{A, W} \kappa^{A, A} \kappa^{S, U} \kappa^{T, V} \kappa_{R S T} \kappa_{U, V, W}+\sum_{\beta, \lambda} \kappa^{A, R} \kappa^{A, W} \kappa^{s, t} \kappa^{A, U} \kappa^{A, V} \kappa_{R s t} \kappa_{U, V, W} \\
& +6 \sum_{\beta, \lambda} \kappa^{A, R} \kappa^{A, W} \kappa^{A, S} \kappa^{t, u} \kappa^{A, V} \kappa_{R S t} \kappa_{u, V, W}+3 \sum_{\beta, \lambda} \kappa^{A, R} \kappa^{A, W} \kappa^{A, A} \kappa^{s, u} \kappa^{T, V} \kappa_{R s T} \kappa_{u, V, W} \\
& +3 \sum_{\beta, \lambda} \kappa^{A, R} \kappa^{A, W} \kappa^{A, A} \kappa^{S, U} \kappa^{t, v} \kappa_{R S t} \kappa_{U, v, W}+3 \sum_{\beta, \lambda} \kappa^{A, R} \kappa^{A, W} \kappa^{A, A} \kappa^{s, u} \kappa^{t, v} \kappa_{R s t} \kappa_{u, v, W}
\end{aligned}
$$

e

$$
\begin{aligned}
A_{32 \lambda}^{4}= & -\left[\sum_{\lambda} \kappa^{A, R} \kappa^{S, T}\left(2 \kappa_{R S, T}+\kappa_{R S T}\right)\right]\left[\sum_{\lambda} \kappa^{A, U} \kappa^{A, V} \kappa^{A, W} \kappa_{U, V, W}\right] \\
& -\left[\sum_{\beta, \lambda} \kappa^{A, R} \kappa^{s, t}\left(2 \kappa_{R s, t}+\kappa_{R s t}\right)\right]\left[\sum_{\lambda} \kappa^{A, U} \kappa^{A, V} \kappa^{A, W} \kappa_{U, V, W}\right] .
\end{aligned}
$$


Substituindo os cumulantes do Apêndice A, invertendo a ordem dos somatórios e rearranjando os termos, obtemos

$$
\begin{aligned}
A_{32 \lambda}^{1}= & 2 \sum_{\ell=1}^{n}\left\{\left(\sum_{R=1}^{q} \kappa^{A, R} s_{R \ell}\right)\left(\sum_{S, T=1}^{q} s_{\ell S} \kappa^{S, T} s_{T \ell}\right)\left(-d_{2 \ell} \phi_{1 \ell} \phi_{2 \ell}\right)\right\} \\
& \times \sum_{m=1}^{n}\left\{\left(\sum_{U=1}^{q} \kappa^{A, U} s_{U m}\right)\left(\sum_{V=1}^{q} \kappa^{A, V} s_{V m}\right)\left(\sum_{W=1}^{q} \kappa^{A, W} s_{W m}\right)\left(-d_{3 m} \phi_{1 m}^{3}\right)\right\} \\
& +6 \sum_{\ell, m=1}^{n}\left\{\left(\sum_{R=1}^{q} \kappa^{A, R} s_{R \ell}\right)\left(\sum_{U=1}^{q} \kappa^{A, U} s_{U \ell}\right)\left(-d_{2 \ell} \phi_{1 \ell} \phi_{2 \ell}\right)\left(\sum_{S, T=1}^{q} s_{\ell S} \kappa^{S, T} s_{T m}\right)\right. \\
& \left.\times\left(-d_{3 m} \phi_{1 m}^{3}\right)\left(\sum_{V=1}^{q} s_{m V} \kappa^{V, A}\right)\left(\sum_{W=1}^{q} s_{m W} \kappa^{W, A}\right)\right\} \\
& +2 \sum_{\ell=1}^{n}\left\{\left(\sum_{R=1}^{q} \kappa^{A, R} s_{R \ell}\right)\left(\sum_{s, t=1}^{p} x_{\ell s} \kappa^{s, t} x_{t \ell}\right)\left(\phi_{1 \ell} w_{\ell}\right)\right\} \\
& \times \sum_{m=1}^{n}\left\{\left(\sum_{U=1}^{q} \kappa^{A, U} s_{U m}\right)\left(\sum_{V=1}^{q} \kappa^{A, V} s_{V m}\right)\left(\sum_{W=1}^{q} \kappa^{A, W} s_{W m}\right)\left(-d_{3 m} \phi_{1 m}^{3}\right)\right\} \\
A_{32 \lambda}^{2}=6 \sum_{\ell=1}^{n} & \left\{\left(\sum_{R=1}^{q} \kappa^{A, R} s_{R \ell}\right)\left(\sum_{S=1}^{q} \kappa^{A, S} s_{S \ell}\right)\left(-d_{3 \ell} \phi_{1 \ell}^{2} \phi_{2 \ell}\right)\left(\sum_{T=1}^{q} s_{\ell T} \kappa^{T, A}\right)\left(\sum_{U=1}^{q} S_{\ell U} \kappa^{U, A}\right)\right\} \\
+ & 6 \kappa^{A, A} \sum_{\ell=1}^{n}\left\{\left(\sum_{R=1}^{q} \kappa^{A, A} \sum_{\ell=1}^{n}\left\{\left(\sum_{R=1}^{q} \kappa_{R \ell}^{A, R} s_{R \ell}\right)\left(-\phi_{1 \ell}^{2} \phi_{\ell}^{-1} w_{\ell}\right)\left(\sum_{s, t=1}^{p} x_{\ell s}^{2} \kappa^{s, t} x_{t \ell}\right)\left(\sum_{U=1}^{q} s_{\ell U} \kappa^{U, A}\right)\right\}\right.\right.
\end{aligned}
$$




$$
\begin{aligned}
A_{32 \lambda}^{3}= & \sum_{\ell=1}^{n}\left\{\left(\sum_{R=1}^{q} \kappa^{A, R} s_{R \ell}\right)\left(\sum_{S, T=1}^{q} s_{\ell S} \kappa^{S, T} s_{T \ell}\right)\left(d_{3 \ell} \phi_{1 \ell}^{3}+3 d_{2 \ell} \phi_{1 \ell} \phi_{2 \ell}\right)\right\} \\
& \times \sum_{m=1}^{n}\left\{\left(\sum_{U=1}^{q} \kappa^{A, U} s_{U m}\right)\left(\sum_{V=1}^{q} \kappa^{A, V} s_{V m}\right)\left(\sum_{W=1}^{q} \kappa^{A, W} s_{W m}\right)\left(-d_{3 m} \phi_{1 m}^{3}\right)\right\} \\
& +6 \sum_{\ell, m=1}^{n}\left\{\left(\sum_{R=1}^{q} \kappa^{A, R} s_{R \ell}\right)\left(\sum_{S=1}^{q} \kappa^{A, S} s_{S \ell}\right)\left(d_{3 \ell} \phi_{1 \ell}^{3}+3 d_{2 \ell} \phi_{1 \ell} \phi_{2 \ell}\right)\left(\sum_{T, U=1}^{q} S_{\ell T} \kappa^{T, U} s_{U m}\right)\right. \\
& \left.\times\left(-d_{3 m} \phi_{1 m}^{3}\right)\left(\sum_{V=1}^{q} s_{m V} \kappa^{V, A}\right)\left(\sum_{W=1}^{q} s_{m W} \kappa^{W, A}\right)\right\} \\
& +3 \kappa^{A, A} \sum_{\ell=1}^{n}\left\{\left(\sum_{R=1}^{q} \kappa^{A, R} s_{R \ell}\right)\left(d_{3 \ell} \phi_{1 \ell}^{3}+3 d_{2 \ell} \phi_{1 \ell} \phi_{2 \ell}\right)\left(\sum_{S, U=1}^{q} s_{\ell S} \kappa^{S, U} s_{U m}\right)\left(\sum_{T, V=1}^{q} s_{\ell T} \kappa^{T, V} s_{V m}\right)\right. \\
& +\sum_{\ell=1}^{n}\left\{\left(\sum_{R=1}^{q} \kappa^{A, R} s_{R \ell}\right)\left(\sum_{s, t=1}^{p} x_{\ell s} \kappa^{s, t} x_{t \ell}\right)\left(-\phi_{1 \ell} w_{\ell}\right)\right\} \\
& \times \sum_{m=1}^{n}\left\{\left(\sum_{U=1}^{q} \kappa^{A, U} s_{U m}\right)\left(\sum_{V=1}^{q} \kappa^{A, V} s_{V m}\right)\left(\sum_{W=1}^{q} \kappa^{A, W} s_{W m}\right)\left(-d_{3 m} \phi_{1 m}^{3}\right)\right\} \\
& +3 \kappa^{A, A} \sum_{\ell=1}^{n}\left\{\left(\sum_{R=1}^{q} \kappa^{A, R} s_{R \ell}\right)\left(-\phi_{1 \ell} w_{\ell}\right)\left(\sum_{s, u=1}^{p} x_{\ell s} \kappa^{s, u} x_{u m}\right)\left(\sum_{t, v=1}^{p} x_{\ell t} \kappa^{t, v} x_{v m}\right)\right. \\
& \left.\times\left(-\phi_{1 \ell} w_{\ell}\right)\left(\sum_{W=1}^{q} s_{m W} \kappa^{W, A}\right)\right\}
\end{aligned}
$$

e

$$
\begin{aligned}
A_{32 \lambda}^{4}= & -\sum_{\ell=1}^{n}\left\{\left(\sum_{R=1}^{q} \kappa^{A, R} s_{R \ell}\right)\left(\sum_{S, T=1}^{q} s_{\ell S} \kappa^{S, T} s_{T \ell}\right)\left(d_{3 \ell} \phi_{1 \ell}^{3}+d_{2 \ell} \phi_{1 \ell} \phi_{2 \ell}\right)\right\} \\
& \times \sum_{m=1}^{n}\left\{\left(\sum_{U=1}^{q} \kappa^{A, U} s_{U m}\right)\left(\sum_{V=1}^{q} \kappa^{A, V} s_{V m}\right)\left(\sum_{W=1}^{q} \kappa^{A, W} s_{W m}\right)\left(-d_{3 m} \phi_{1 m}^{3}\right)\right\} \\
& -\sum_{\ell=1}^{n}\left\{\left(\sum_{R=1}^{q} \kappa^{A, R} s_{R \ell}\right)\left(\sum_{s, t=1}^{q} x_{\ell s} \kappa^{s, t} x_{t \ell}\right)\left(\phi_{1 \ell} w_{\ell}\right)\right\} \\
& \times \sum_{m=1}^{n}\left\{\left(\sum_{U=1}^{q} \kappa^{A, U} s_{U m}\right)\left(\sum_{V=1}^{q} \kappa^{A, V} s_{V m}\right)\left(\sum_{W=1}^{q} \kappa^{A, W} s_{W m}\right)\left(-d_{3 m} \phi_{1 m}^{3}\right)\right\} .
\end{aligned}
$$


Escrevendo as expressões em notação matricial, obtemos

$$
\begin{aligned}
& \mathbf{A}_{\mathbf{3 2 \lambda}}^{\mathbf{1}}=2\left[\boldsymbol{P}_{\lambda} \boldsymbol{Z}_{\lambda d} \boldsymbol{D}_{2} \boldsymbol{\Phi}_{1} \boldsymbol{\Phi}_{2} \mathbf{1}\right]\left[\left(\boldsymbol{P}_{\lambda} \odot \boldsymbol{P}_{\lambda} \odot \boldsymbol{P}_{\lambda}\right) \boldsymbol{D}_{3} \boldsymbol{\Phi}_{1}^{3}\right]^{\top} \\
& +6\left[\left(\boldsymbol{P}_{\lambda} \odot \boldsymbol{P}_{\lambda}\right) \boldsymbol{D}_{2} \boldsymbol{\Phi}_{1} \boldsymbol{\Phi}_{2} \boldsymbol{Z}_{\lambda} \boldsymbol{D}_{3} \boldsymbol{\Phi}_{1}^{3}\left(\boldsymbol{P}_{\lambda} \odot \boldsymbol{P}_{\lambda}\right)^{\top}\right]_{d} \\
& -2\left[\boldsymbol{P}_{\lambda} \boldsymbol{Z}_{\beta d} \boldsymbol{\Phi}_{1} \boldsymbol{W} \mathbf{1}\right]\left[\left(\boldsymbol{P}_{\lambda} \odot \boldsymbol{P}_{\lambda} \odot \boldsymbol{P}_{\lambda}\right) \boldsymbol{D}_{3} \boldsymbol{\Phi}_{1}^{3}\right]^{\top} \\
& \mathbf{A}_{\mathbf{3 2 \lambda}}^{\mathbf{2}}=-6\left[\left(\boldsymbol{P}_{\lambda} \odot \boldsymbol{P}_{\lambda}\right) \boldsymbol{D}_{3} \boldsymbol{\Phi}_{1}^{2} \boldsymbol{\Phi}_{2}\left(\boldsymbol{P}_{\lambda} \odot \boldsymbol{P}_{\lambda}\right)^{\top}\right]_{d} \\
& -6\left[\boldsymbol{K}_{\lambda}^{-1}\right]_{d}\left[\boldsymbol{P}_{\lambda} \boldsymbol{D}_{3} \boldsymbol{\Phi}_{1}^{2} \boldsymbol{\Phi}_{2} \boldsymbol{Z}_{\lambda d} \boldsymbol{P}_{\lambda}^{\top}\right]_{d} \\
& -6\left[\boldsymbol{K}_{\lambda}^{-1}\right]_{d}\left[\boldsymbol{P}_{\lambda} \boldsymbol{\Phi}_{1}^{2} \boldsymbol{\Phi}^{-1} \boldsymbol{W} \boldsymbol{Z}_{\beta d} \boldsymbol{P}_{\lambda}^{\top}\right]_{d} \\
& \mathbf{A}_{\mathbf{3 2 \lambda}}^{\mathbf{3}}=-\left[\boldsymbol{P}_{\lambda} \boldsymbol{Z}_{\lambda d}\left(\boldsymbol{D}_{3} \boldsymbol{\Phi}_{1}^{3}+3 \boldsymbol{D}_{2} \boldsymbol{\Phi}_{1} \boldsymbol{\Phi}_{2}\right) \mathbf{1}\right]\left[\left(\boldsymbol{P}_{\lambda} \odot \boldsymbol{P}_{\lambda} \odot \boldsymbol{P}_{\lambda}\right) \boldsymbol{D}_{3} \boldsymbol{\Phi}_{1}^{3} \mathbf{1}\right]^{\top} \\
& -6\left[\left(\boldsymbol{P}_{\lambda} \odot \boldsymbol{P}_{\lambda}\right)\left(\boldsymbol{D}_{3} \boldsymbol{\Phi}_{1}^{3}+3 \boldsymbol{D}_{2} \boldsymbol{\Phi}_{1} \boldsymbol{\Phi}_{2}\right) \boldsymbol{Z}_{\lambda} \boldsymbol{D}_{3} \boldsymbol{\Phi}_{1}^{3}\left(\boldsymbol{P}_{\lambda} \odot \boldsymbol{P}_{\lambda}\right)^{\top}\right]_{d} \\
& -3\left[\boldsymbol{K}_{\lambda}^{-1}\right]_{d}\left[\boldsymbol{P}_{\lambda}\left(\boldsymbol{D}_{3} \boldsymbol{\Phi}_{1}^{3}+3 \boldsymbol{D}_{2} \boldsymbol{\Phi}_{1} \boldsymbol{\Phi}_{2}\right)\left(\boldsymbol{Z}_{\lambda} \odot \boldsymbol{Z}_{\lambda}\right) \boldsymbol{D}_{3} \boldsymbol{\Phi}_{1}^{3} \boldsymbol{P}_{\lambda}^{\top}\right]_{d} \\
& +\left[\boldsymbol{P}_{\lambda} \boldsymbol{Z}_{\beta d} \boldsymbol{\Phi}_{1} \boldsymbol{W} \mathbf{1}\right]\left[\left(\boldsymbol{P}_{\lambda} \odot \boldsymbol{P}_{\lambda} \odot \boldsymbol{P}_{\lambda}\right) \boldsymbol{D}_{3} \boldsymbol{\Phi}_{1}^{3} \mathbf{1}\right]^{\top} \\
& +3\left[\boldsymbol{K}_{\lambda}^{-1}\right]_{d}\left[\boldsymbol{P}_{\lambda} \boldsymbol{\Phi}_{1} \boldsymbol{W}\left(\boldsymbol{Z}_{\beta} \odot \boldsymbol{Z}_{\beta}\right) \boldsymbol{\Phi}_{1} \boldsymbol{W} \boldsymbol{P}_{\lambda}^{\top}\right]_{d}
\end{aligned}
$$

e

$$
\begin{aligned}
\mathbf{A}_{\mathbf{3 2 \lambda}}^{4}= & {\left[\boldsymbol{P}_{\lambda} \boldsymbol{Z}_{\lambda d}\left(\boldsymbol{D}_{3} \boldsymbol{\Phi}_{1}^{3}+\boldsymbol{D}_{2} \boldsymbol{\Phi}_{1} \boldsymbol{\Phi}_{2}\right) \mathbf{1}\right]\left[\left(\boldsymbol{P}_{\lambda} \odot \boldsymbol{P}_{\lambda} \odot \boldsymbol{P}_{\lambda}\right) \boldsymbol{D}_{3} \boldsymbol{\Phi}_{1}^{3} \mathbf{1}\right]^{\top} } \\
& +\left[\boldsymbol{P}_{\lambda} \boldsymbol{Z}_{\beta d} \boldsymbol{\Phi}_{1} \boldsymbol{W} \mathbf{1}\right]\left[\left(\boldsymbol{P}_{\lambda} \odot \boldsymbol{P}_{\lambda} \odot \boldsymbol{P}_{\lambda}\right) \boldsymbol{D}_{3} \boldsymbol{\Phi}_{1}^{3} \mathbf{1}\right]^{\top}
\end{aligned}
$$




\section{Referências Bibliográficas}

Barroso et al. (2013) Lúcia P. Barroso, Denise A. Botter e Gauss M. Cordeiro. Second-order covariance matrix formula for heteroskedastic generalized linear models. Communications in Statistics - Theory and Methods, 42(9):1618-1627. Citado na pág. 2, 7, 8, 11, 12

Botter e Cordeiro (1997) Denise A. Botter e Gauss M. Cordeiro. Bartlett corrections for generalized linear models with dispersion covariates. Communications in Statistics Theory and Methods, 26(2):279-307. Citado na pág. 2

Botter e Cordeiro (1998) Denise A. Botter e Gauss M. Cordeiro. Improved estimators for generalized linear models with dispersion covariates. Journal of Statistical Computation and Simulation, 62(1-2):91-104. Citado na pág. 2, 13

Bowman e Shenton (1998) K. O. Bowman e L. R. Shenton. Asymptotic skewness and the distribution of maximum likelihood estimators. Communications in Statistics - Theory and Methods, 27(11):2743-2760. Citado na pág. 2, 60, 61, 70

Bowman e Shenton (1999) K.O. Bowman e L.R. Shenton. The asymptotic kurtosis for maximum likelihood estimators. Communications in Statistics - Theory and Methods, 28 (11):2641-2654. Citado na pág. 2, 69, 71

Cordeiro (1983) Gauss M. Cordeiro. Improved likelihood ratio statistics for generalized linear models. Journal of the Royal Statistical Society. Series B (Methodological), 43(3): 404-413. Citado na pág. 2

Cordeiro (1987) Gauss M. Cordeiro. On the corrections to the likelihood ratio statistics. Biometrika, 74(2):265-274. Citado na pág. 2

Cordeiro (2004) Gauss M. Cordeiro. Second-order covariance matrix ofmaximum likelihood estimates in generalized linear models. Statistics \& Probability Letters, 66:153-160. Citado na pág. 2, 7, 8, 12

Cordeiro (1991) Gauss M. Cordeiro. Introdução à Teoria Assintótica. Livro texto do $22^{\circ}$ Colóquio Brasileiro de Matemática, IMPA, Rio de Janeiro, RJ. Citado na pág. 94

Cordeiro e Ferrari (1991) Gauss M. Cordeiro e Silvia L. P. Ferrari. A modified score test statistic having chi-squared distribution to order $n^{-1}$. Biometrika, 78(3):573-582. Citado na pág. 2

Cordeiro e McCullagh (1991) Gauss M. Cordeiro e Peter McCullagh. Bias correction in generalized linear models. Journal of the Royal Statistical Society. Series B (Methodological), 53(3):629-643. Citado na pág. 1 
Cordeiro e Santana (2008) Gauss M. Cordeiro e Rosangela G. Santana. Covariance matrix formula for exponential nonlinear models. Communications in Statistics - Theory and Methods, 37(17):2724-2734. Citado na pág. 7

Cordeiro et al. (1993) Gauss M. Cordeiro, Silvia L. P. Ferrari e Gilberto A. Paula. Improved score tests for generalized linear models. Journal of the Royal Statistical Society. Series $B, 55(3): 661-674$. Citado na pág. 2

Cordeiro et al. (2003) Gauss M. Cordeiro, Denise A. Botter, Lúcia P. Barroso e Silvia L. P. Ferrari. Three corrected score tests for generalized linear models with dispersion covariates. Communications in Statistics - Theory and Methods, 57(4):391-409. Citado na pág. 2

Cordeiro et al. (2006) Gauss M. Cordeiro, Lúcia P. Barroso e Denise A. Botter. Covariance matrix formula for generalized linear models with unknown dispersion. Communications in Statistics - Theory and Methods, 35(1):113-120. Citado na pág. 2, 7, 8, 12

Cordeiro et al. (2014) Gauss M. Cordeiro, Denise A. Botter, Alexsandro B. Cavalcanti e Lúcia P. Barroso. Covariance matrix of the bias-corrected maximum likelihood estimator in generalized linear models. Statistical Papers, 55(3):643-652. Citado na pág. 2, 13, 17

Cordeiro e Cordeiro (2001) Hérbetes H. Cordeiro e Gauss M. Cordeiro. Skewness for parameters in generalized linear models. Communications in Statistics - Theory and Methods, 30(7):1317-1334. Citado na pág. 2, 60, 61, 62

Cox e Hinkley (1974) D. R. Cox e D. V. Hinkley. Theoretical Statistics. London: Chapman and Hall. Citado na pág. 3

Cox e Reid (1987) D. R. Cox e N. Reid. Parameter orthogonality and approximate conditional inference (with discussion. Journal of the Royal Statistical Society B, 49(1): 1-39. Citado na pág. 4

Cox e Snell (1968) D. R. Cox e E. J. Snell. A general definition of residuals. Journal of the Royal Statistical Society. Series B (Methodological), 30(2):248-275. Citado na pág. 1

Cribari-Neto e Ferrari (1995) Francisco Cribari-Neto e Silvia L. P. Ferrari. Second order asymptotics for score tests in generalised linear models. Journal of the Royal Statistical Society. Series B, 82(2):426-432. Citado na pág. 2

Doornik (2006) Jurgen A. Doornik. Ox: An Object-Oriented Matrix Language. Timberlake Consultants Press, London, 5th ed. Citado na pág. 6, 20, 41, 63, 85

Efron e Tibshirani (1993) Bradley Efron e Robert J. Tibshirani. An Introduction to the Bootstrap. Chapman \& Hall, New York. Citado na pág. 20

Ferrari e Cysneiros (2008) Silvia L. P. Ferrari e Audrey H. M. A. Cysneiros. Skovgaard's adjustment to likelihood ratio tests in exponential family nonlinear models. Statistics and Probability Letters, 78:3047-3055. Citado na pág. 2, 41

Kendall e Stuart (1977) Maurice G. Kendall e Alan Stuart. The Advanced Theory of Statistic. Griffin, London, 4th ed. Citado na pág. 60 
Lawley (1956) D. N. Lawley. A general method for approximating to the distribution of likelihood ratio criteria. Biometrika, 43(3/4):295-303. Citado na pág. 2, 8

Lemonte (2011) Artur J. Lemonte. Local power of some tests in exponential family nonlinear models. Journal of Statistical Planning and Inference, 141(5):1981-1989. Citado na pág. 31

Lemonte (2012) Artur J. Lemonte. Local power properties of some asymptotic tests in symmetric linear regression models. Journal of Statistical Planning and Inference, 142(5): 1178-1188. Citado na pág. 31

Lemonte (2013) Artur J. Lemonte. Nonnull asymptotic distributions of the lr, wald, score and gradient statistics in generalized linear models with dispersion covariates. Statistics: A Journal of Theoretical and Applied Statistics, 47(6):1249-1265. Citado na pág. 31, 91

Lemonte (2014a) Artur J. Lemonte. Nonnull asymptotic distributions of some criteria in censored exponential regression. Communications in Statistics - Theory and Methods, 43 (15):3314-3328. Citado na pág. 31

Lemonte (2014b) Artur J. Lemonte. On local power properties of the lr, wald, score and gradient tests in nonlinear mixed-effects models. Annals of the Institute of Statistical Mathematics. URL DOI:10.1007/s10463-014-0478-5. Citado na pág. 31

Lemonte e Ferrari (2011) Artur J. Lemonte e Silvia L. P. Ferrari. Size and power properties of some tests in the birnbaum-saunders regression model. Computational Statistics and Data Analysis, 55(2):1109-1117. Citado na pág. 31

Lemonte e Ferrari (2012a) Artur J. Lemonte e Silvia L. P. Ferrari. The local power of the gradient test. Annals of the Institute of Statistical Mathematics, 64(2):373-381. Citado na pág. 30

Lemonte e Ferrari (2012b) Artur J. Lemonte e Silvia L. P. Ferrari. Local power and size properties of the lr, wald, score and gradient tests in dispersion models. Statistical Methodology, 9(5):537-554. Citado na pág. 31

Lemonte (2016) Artur José Lemonte. The Gradient Test: Another Likelihood-Based Test. London: Academic Press, 1 ed. Citado na pág. 2, 31

Magalhães (2016) Tiago Maia Magalhães. Refinamento de propriedades assintóticas do estimador de máxima verossimilhança. Tese de Doutorado, Instituto de Matemática e Estatística, Universidade de São Paulo, Brasil. Citado na pág. 7

Montgomery et al. (2001) D. C. Montgomery, E. A. Peck e G. G. Vining. Introduction to Linear Regression Analysis. John Wiley, New York, third ed. Citado na pág. 91

Nelder e Wedderburn (1972) J. A. Nelder e R. W. M. Wedderburn. Generalized linear models. Journal of the Royal Statistics Society A, 135(3):370-384. Citado na pág. 1

Nocedal e Wright (2006) Jorge Nocedal e Stephen Wright. Numerical Optimization. Springer, second ed. Citado na pág. 4, 20, 41

Peers e Iqbal (1985) H. W. Peers e Mohammad Iqbal. Asympotic expansions for cofidence limits in the presence of nuisance parameters, with applications. Journal of the Royal Statistical Society B, 47:547-554. Citado na pág. 7, 13 
Pregibon (1984) Daryl Pregibon. Review of generalized linear models. The Annals of Statistics, 12(4):1589-1596. Citado na pág. 1

Rao (1947) C. Radhakrishna Rao. Large sample tests of statistical hypotheses concerning several parameters with applications to problems of estimation. Mathematical Proceedings of the Cambridge Philosophical Society, 44(01):50-57. Citado na pág. 2, 30

Rao (2005) C. Radhakrishna Rao. Score test: historical review and recent developments. Em N. Balakrishnan, N. Kannan e H. N. Nagaraja, editors, Advances in Ranking and Selection, Multiple Comparisons, and Reliability. Citado na pág. 30

Rocha et al. (2010) Andréa V. Rocha, Alexandre B. Simas e Gauss M. Cordeiro. Secondorder asymptotic expressions for the covariance matrix of maximum likelihood estimators in dispersion models. Statistics \& Probability Letters, 80(7-8):718-725. Citado na pág. 7

Shenton e Bowman (1977) L.R. Shenton e K.O. Bowman. Maximum likelihood estimation in small samples. Charles Griffin and Co., Ltd., England. Citado na pág. 7, 13

Simonoff e Tsai (1994) J. S. Simonoff e C. L. Tsai. Use of modified profile likelihood for improved tests of constancy of variance in regression. Applied Statistics, 43(2):357-370. Citado na pág. 28, 57

Skovgaard (2001) Ib M. Skovgaard. Likelihood asymptotics. Scandinavian Journal of Statistics, 28(1):3-32. Citado na pág. 2

Smyth (1989) Gordon K. Smyth. Generalized linear models with varying dispersion. Journal of the Royal Statistical Society. Series B (Methodological), 51(1):47-60. Citado na pág. i, ii, 1

Terrell (2002) George R. Terrell. The gradient statistic. Computing Science and Statistics, 34:206-215. Citado na pág. 2, 30

Vargas et al. (2013) Tiago M. Vargas, Silvia L. P. Ferrari e Artur J. Lemonte. Gradient statistic: higher-order asymptotics and bartlett-type correction. Electronic Journal of Statistics, 7:43-61. Citado na pág. 2, 31, 32

Vargas et al. (2014) Tiago M. Vargas, Silvia L. P. Ferrari e Artur J. Lemonte. Improved likelihood inference in generalized linear models. Computational Statistics and Data Analysis, 74:110-124. Citado na pág. 31

Wald (1943) Abraham Wald. Tests of statistical hypotheses concerning several parameters when the number of observations is large. Transactions of the American Mathematical Society, 54:426-482. Citado na pág. 2, 30

Wilks (1938) S. S. Wilks. The large-sample distribution of the likelihood ratio for testing composite hypotheses. Annals of Mathematical Statistics, 9(1):60-62. Citado na pág. 2, 30 